\title{
Decaying Dark Matter models at colliders
}

\author{
Dissertation \\ zur Erlangung des mathematisch-naturwissenschaftlichen Doktorgrades \\ "Doctor rerum naturalium" \\ der Georg-August-Universität Göttingen \\ im Promotionsprogramm ProPhys \\ der Georg-August University School of Science (GAUSS)
}

vorgelegt von

Federico Dradi

aus Cusco (Peru)

Göttingen, 2015 


\section{Betremungsausschuss}

Prof. Dr. Laura Covi, Physik, Institut für Theoretische Physik Jun. -Prof. Dr Steffen Schumann, Physik, II Institut für Physik

Prof. Dr. Karl-Henning Rehren, Physik, Institut für Theoretische Physik

\section{Mitglieder der Prïfungskommission}

Referentin: Prof. Dr. Laura Covi, Physik, Institut für Theoretische Physik

Korreferent: Jun. -Prof. Dr Steffen Schumann, Physik, II Institut für Physik

Weitere Mitglieder der Prüfungskommission:

Prof. Dr. Arnulf Quadt, Physik, II Institut für Physik

Prof. Dr. Jens Niemeyer, Physik, Institut für Astrophysik

PD Dr. Riccardo Catena, Physik, Institut für Theoretische Physik

Prof. Dr. Karl-Henning Rehren, Physik, Institut für Theoretische Physik

Tag der mündlichen Prüfung: 


\section{UNIVERSITY OF GÖTTINGEN}

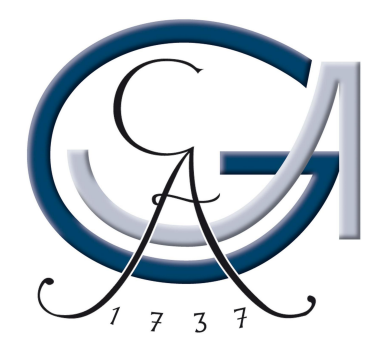

Doctoral Thesis

\section{Decaying Dark Matter models at colliders}

Author:

Federico DRADI
Supervisor:

Prof. Dr. Laura Covi

Second Supervisor:

Prof. Dr. Steffen SchumanN

A thesis submitted in fulfilment of the requirements

for the degree of Doctor of Philosophy

in the

Particle Theory group

Institute for Theoretical Physics

July 2015 

"Do one thing every day that scares you."

Eleanor Roosevelt

US diplomat \& reformer (1884 - 1962) 



\section{Contents}

$\begin{array}{ll}\text { Contents } & \text { v }\end{array}$

$\begin{array}{lr}\text { List of Figures } & \text { vii }\end{array}$

$\begin{array}{ll}\text { List of Tables } & \text { ix }\end{array}$

$\begin{array}{ll}\text { Abbreviations } & \text { xi }\end{array}$

$\begin{array}{lr}\text { Introduction } & 1\end{array}$

1 Models for Dark Matter particles and CMS detector 5

1.1 Basics of Supersymmetry . . . . . . . . . . . . . . . . . . 6

1.2 Minimal Supersymmetric Standard Model . . . . . . . . . . . . . . . . . . . 9

1.3 Supergravity ... . . . . . . . . . . . . . . . . . . . . 21

1.4 Minimal Dark Matter Model ....................... 25

1.5 Experiment setup . . . . . . . . . . . . . . . . . . . . . 29

2 Dark Matter $\quad 33$

2.1 A short history of Dark Matter . . . . . . . . . . . . . . . . . . . 34

2.2 Evidences for the existence of Dark Matter . . . . . . . . . . . . . . . 37

2.3 Dark Matter ten-point test . . . . . . . . . . . . . . . . . . . 41

2.4 Introduction to the Standard Cosmology Model . . . . . . . . . . . . . . . . . . . 47

2.5 Dark Matter production . . . . . . . . . . . . . . . . . . . 57

2.6 Gravitino as Dark Matter candidate . . . . . . . . . . . . . . . . . 60

2.7 DM production in the Mininal DM decaying model . . . . . . . . . . . . . . 62

3 Long-Lived stop at LHC $\quad 65$

3.1 Stop NLSP couplings, production and decay channels . . . . . . . . . . . . 66

3.2 Cosmology of Stop NLPS and gravitino LSP . . . . . . . . . . . . . . . . . 69

3.3 Decay of stop NLSP at LHC . . . . . . . . . . . . . . . . . . . . . 75

3.4 LHC reach for the RPC/RPV stop decay . . . . . . . . . . . . . . . . . . . 82

3.5 RPC and RPV stop NLSP decays at LHC . . . . . . . . . . . . . . . . . . 86

3.6 Discussion of results and conclusion . . . . . . . . . . . . . . . . . 90

4 Minimal Decaying DM at LHC 93

4.1 Collider analysis ............................ . . . 94

4.1 Colored scalar . . . . . . . . . . . . . . . . . . 96

4.1.2 EW-charged scalar ...................... 104 
4.2 Discussion of results and conclusions . . . . . . . . . . . . . . . . 111

$5 \quad 3.55 \mathrm{keV}$ DM line at LHC $\quad 119$

5.1 Experimental evidence of the $3.55 \mathrm{keV}$ line . . . . . . . . . . . 120

5.2 Minimal scenario . . . . . . . . . . . . . . . . . . . . . . . . . 121

5.3 Dark matter and Dark radiation scenario . . . . . . . . . . . . . . 126

$5.4 \mathrm{DM}$ as sterile neutrino . . . . . . . . . . . . . . . . . 130

5.5 Conclusions . . . . . . . . . . . . . . . . . . . . . . . 132

Final Conclusions $\quad 135$

$\begin{array}{ll}\text { A Python code for exponential decay } & 137\end{array}$

$\begin{array}{ll}\text { B Mathematica code } & 141\end{array}$

$\begin{array}{lr}\text { C FeynRules code } & 145\end{array}$

$\begin{array}{ll}\text { Bibliography } & 147\end{array}$

$\begin{array}{ll}\text { Acknowledgements } & 147\end{array}$ 


\section{List of Figures}

1.1 1-loop radiative correction to $m_{H}$ due to the fermion couplings $\ldots \ldots \ldots$

1.2 1-loop radiative correction to $m_{H}$ due to the scalar couplings . . . . . . . . . 8

1.3 2-loop renormalization group evolution of $\alpha_{\alpha}^{-1}(Q)$ in SM and MSSM . . . . . . 9

$1.4 \Sigma_{f}$ decay length into DM and one fermion vs. the decay length into only SM states 28

1.5 Layout of two quarters of CMS detector . . . . . . . . . . . . . . 30

2.1 Histogram of Zwicky's citations history . . . . . . . . . . . . . . 36

2.2 Rotational curve of NGC $6503 \ldots \ldots \ldots \ldots \ldots$

2.3 Gravitational lensing of the cluster CL0024+1654 . . . . . . . . . . . . . 39

2.4 Bullet cluster image . . . . . . . . . . . . . . . . . . . 40

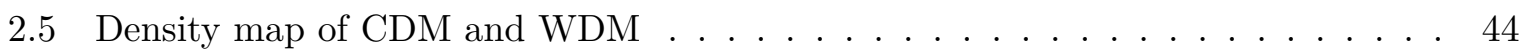

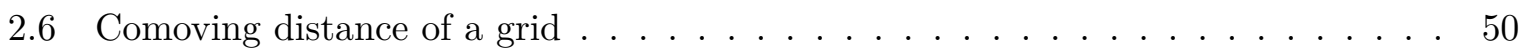

2.7 BBN predictions of the light elements abundances $\ldots \ldots \ldots \ldots \ldots$

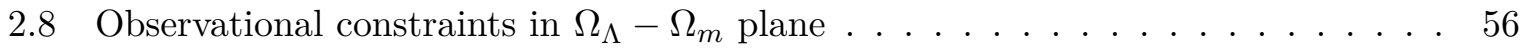

2.9 Evolution of the relic yields for conventional freeze-out and freeze-in . . . . . . . 60

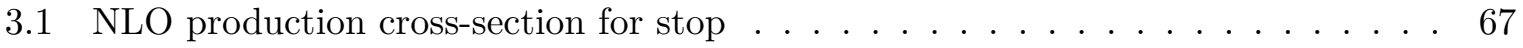

3.2 Kusakabe's BBN constraints . . . . . . . . . . . . . . . . . . 70

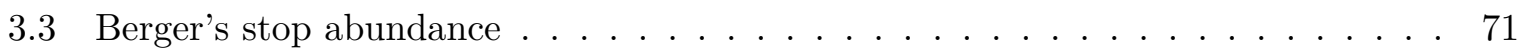

3.4 BBN bounds on the RPC stop NLSP with gravitino LSP scenario . . . . . . . 72

3.5 Plot of $m_{\tilde{t}}\left(m_{3 / 2}\right)$ with cosmological constraints . . . . . . . . . . . . . 74

3.6 Plot of $T_{R}\left(m_{\tilde{t}}\right)$ for $m_{3 / 2}=1 \mathrm{GeV}$ and Plot of $T_{R}^{\max }\left(m_{\tilde{t}}\right) \ldots \ldots \ldots \ldots$

3.7 Distribution of 10,000 stop displaced vertices in the detector plane $(r, z) \ldots \ldots 77$

3.8 Madgraph NLO LHC reach in " $\tau_{\tilde{t}}$ vs $m_{\tilde{t}}$ " plane at $L=\{25,3000\} \mathrm{fb}^{-1} \ldots \ldots .78$

3.9 MadGraph distribution of the factor $\beta \gamma$ for $m_{\tilde{t}}=800 \mathrm{GeV}$. . . . . . . . . . 80

3.10 Semi-analytical NLO LHC reach in " $\tau_{\tilde{t}}$ vs $m_{\tilde{t}}$ " plane at $L=\{25,3000\} \mathrm{fb}^{-1} \ldots 2$

3.11 Semi-analytical NLO LHC reach in " $\tau_{\tilde{t}}$ vs $m_{\tilde{t}}$ " plane for $N=\{1,10,100\}$ and $L=\{25,3000\} \mathrm{fb}^{-1} \ldots \ldots \ldots \ldots \ldots \ldots \ldots$

3.12 Semi-analytical NLO LHC reach in " $\tau_{\tilde{t}}$ vs $m_{\tilde{t}}$ " plane at $L=\{100,300\} \mathrm{fb}^{-1} \ldots 83$

3.13 Comparison of numerical and approximate NLO LHC reach at $L=\{25,3000\} \mathrm{fb}^{-1}$

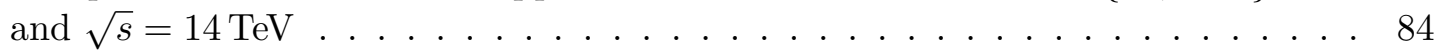

3.14 Comparison of numerical and approximate NLO LHC reach at $L=\{25,3000\} \mathrm{fb}^{-1}$

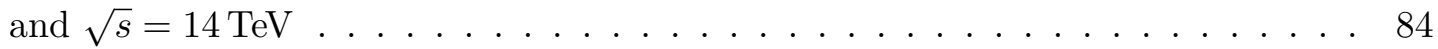

3.15 NLO LHC reach for the RPC $\tilde{t}$ decay at $L=\{25,3000\} \mathrm{fb}^{-1}$ and $\sqrt{s}=14 \mathrm{TeV} \quad$. $\quad 85$

3.16 NLO LHC reach for the RPV $\tilde{t}$ decay at $L=\{25,3000\} \mathrm{fb}^{-1}$ and $\sqrt{s}=14 \mathrm{TeV} \quad$. 86

3.17 Feynman diagram of the 2-body RPV stop decay . . . . . . . . . . . . . . 87

3.18 Feynman diagram of the 4 -body RPC stop decay . . . . . . . . . . . . . 87

3.19 Transverse momentum distribution of $\ell^{+}$for 2-body RPV $\tilde{t}$ decay and 4-body RPC $\tilde{t}$ decay at $m_{\tilde{t}}=800 \mathrm{GeV} \ldots \ldots \ldots \ldots$. . . . . . . . . . 88 
3.20 Transverse mass distributions of the final pair $\ell^{+}-b$ for 2-body RPV $\tilde{t}$ decay and 4-body RPC $\tilde{t}$ decay at $m_{\tilde{t}}=800 \mathrm{GeV} \ldots \ldots \ldots$. . . . . . . . . 89

3.21 Angle distributions of the final pair $\ell^{+}-b$ for 2 -body RPV $\tilde{t}$ decay and 4-body RPC $\tilde{t}$ decay at $m_{\tilde{t}}=800 \mathrm{GeV} \ldots \ldots \ldots \ldots . \ldots \ldots$

$4.1 \Sigma_{d}$ LHC reach in " $\lambda$ vs $\lambda^{\prime \prime}$ " plane at $L=\{300,3000\} \mathrm{fb}^{-1}, m_{\Sigma_{d}}=800 \mathrm{GeV} \ldots . .97$

$4.2 \Sigma_{d}$ LHC reach in " $\lambda$ vs $\lambda^{\prime \prime}$ " plane with contours of the correct DM abundance and ID limit for $m_{\Sigma_{d}}=800 \mathrm{GeV}, L=\{25,300,3000\} \mathrm{fb}^{-1} \ldots \ldots \ldots$. . . . . . 99

$4.3 \Sigma_{d}$ LHC reach in " $\lambda$ vs $\lambda^{\prime}$ " plane with contours of the correct DM abundance and ID limit for $m_{\Sigma_{d}}=\{1600,2200\} \mathrm{GeV}, L=3000 \mathrm{fb}^{-1} \ldots \ldots \ldots \ldots$

$4.4 \Sigma_{d}$ LHC reach in " $\operatorname{Br}\left(\Sigma_{d} \rightarrow \mathrm{DM}\right)$ vs $\Gamma_{\Sigma_{d}}^{-1}$ " at $L=\{25,300\} \mathrm{fb}^{-1}, m_{\Sigma_{d}}=800 \mathrm{GeV} 101$

$4.5 \Sigma_{d}$ LHC reach in "Br $\left(\Sigma_{d} \rightarrow \mathrm{DM}\right)$ vs $\Gamma_{\Sigma_{d}}^{-1}$ " at $L=3000 \mathrm{fb}^{-1}, m_{\Sigma_{d}}=\{800,1600,2200\}$

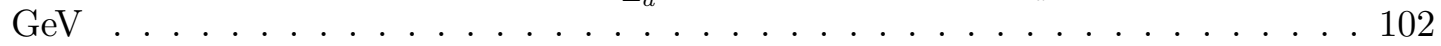

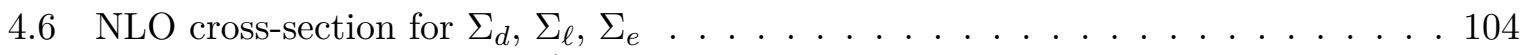

4.7 $\Sigma_{e, \ell}$ LHC reaches in " $\lambda \mathrm{vs} \lambda^{\prime}$ " plane with contours of the correct DM abundance and ID limit for $m_{\Sigma_{e, \ell}}=400 \mathrm{GeV}, L=300 \mathrm{fb}^{-1} \ldots \ldots \ldots \ldots$

$4.8 \Sigma_{e, \ell}$ LHC reaches in " $\lambda \mathrm{vs} \lambda^{\prime}$ " plane with contours of the correct DM abundance and ID limit for $m_{\Sigma_{e, \ell}}=800 \mathrm{GeV}, L=300 \mathrm{fb}^{-1} \ldots \ldots \ldots \ldots$

$4.9 \Sigma_{e, \ell}$ LHC reaches in $" \operatorname{Br}\left(\Sigma_{e, \ell} \rightarrow \mathrm{DM}\right)$ vs $\Gamma_{\Sigma_{d}}^{-1}$ " at $L=300 \mathrm{fb}^{-1}, m_{\Sigma_{e, \ell}}=400 \mathrm{GeV} .107$

$4.10 \Sigma_{e, \ell}$ LHC reaches in $" \operatorname{Br}\left(\Sigma_{e, \ell} \rightarrow \mathrm{DM}\right)$ vs $\Gamma_{\Sigma_{d}}^{-1}$ " at $L=300 \mathrm{fb}^{-1}, m_{\Sigma_{e, \ell}}=800 \mathrm{GeV} .107$

4.11 Comparison of CLIC and LHC cross sections of $\Sigma_{\ell}$ production . . . . . . . 111

4.12 Summary of the possible $\Sigma_{d}$ LHC signals at $L=300 \mathrm{fb}^{-1}, m_{\psi}=\{10,100\} \mathrm{GeV}$. 113

4.13 Summary of the possible $\Sigma_{\ell}$ LHC signals at $L=300 \mathrm{fb}^{-1}, m_{\psi}=\{10,100\} \mathrm{GeV}$. . 113

4.14 Summary of the possible $\Sigma_{\ell}$ CLIC signals at $L=300 \mathrm{fb}^{-1}, m_{\psi}=\{10,100\} \mathrm{GeV}$. 115

4.15 Summary of the possible $\Sigma_{d}$ LHC signals at $L=300 \mathrm{fb}^{-1}, m_{\psi}=1 \mathrm{GeV} \ldots \ldots . .115$

5.2 DM 2-body decay into $\gamma$ and $\nu$ with the loop induced by $\Sigma_{d} \ldots \ldots \ldots$. . . 121

5.3 Summary plot for the minimal decaying dark matter scenario . . . . . . . . . . 124

5.4 DM 2-body decay into $\gamma$ and $\chi$ with the loop induced by scalar-mixing . . . . . . 127

5.5 Summary plot for the Dark Radiation scenario . . . . . . . . . . . . . . . 129

5.6 Diagrams contributing at one-loop to the DM 2-body decay into $\gamma$ and $\nu$ induced by $W \ldots \ldots \ldots \ldots \ldots \ldots 130$

5.7 Constraints on the sterile neutrino model from the literature . . . . . . . . 131

A.1 1 MG-output-event for $m_{\tilde{t}}=800 \mathrm{GeV}, \Gamma_{\tilde{t}}=10^{-10} \mathrm{GeV} \ldots \ldots \ldots \ldots$ 


\section{List of Tables}

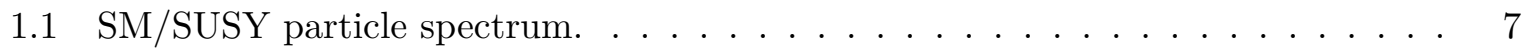

1.2 Gauge supermultiplets of the MSSM. . . . . . . . . . . . . . . . . 10

1.3 Chiral supermultiplets of the MSSM. . . . . . . . . . . . . . 11

1.4 Gravity supermultiplet. . . . . . . . . . . . . . . 21

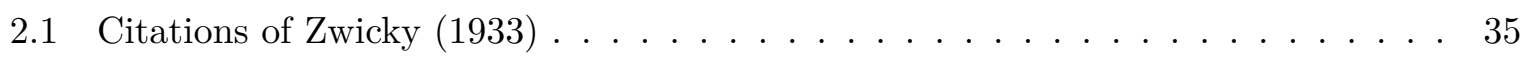

2.2 Cosmological constants derived from Planck 2015 data . . . . . . . . . . . . 53

3.1 Coincidence counting for stop and antistop, $\tau_{\tilde{t}}=\left\{3.254 \times 10^{-9}, 3.254 \times 10^{-7}\right\} \mathrm{s}$,

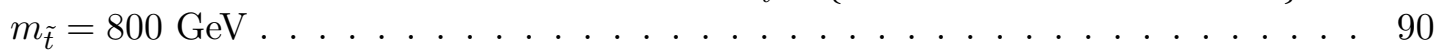

3.2 Coincidence counting for stop and antistop, $\tau_{\tilde{t}}=\left\{3.254 \times 10^{-9}, 3.254 \times 10^{-7}\right\} \mathrm{s}$,

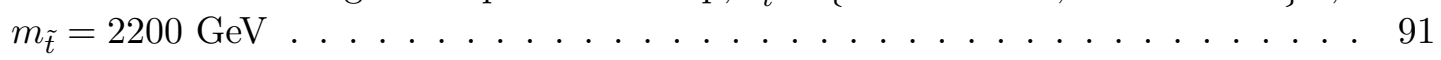

$4.1 \quad \Sigma_{d}$ benchmarks at $m_{\Sigma_{d}}=800 \mathrm{GeV}, x=0.01, \lambda=1.8 \times 10^{-11}, \lambda^{\prime}=5.5 \times 10^{-10} .100$

$4.2 \quad \Sigma_{d}$ benchmarks at $m_{\Sigma_{d}}=1 \mathrm{TeV}, x=0.5, \lambda=1.2 \times 10^{-12}, \lambda^{\prime}=3.6 \times 10^{-12} \ldots 103$

$4.3 \quad \Sigma_{d}$ benchmarks at $m_{\Sigma_{d}}=1 \mathrm{TeV}, x=10^{-6}, \lambda=6.5 \times 10^{-10}, \lambda^{\prime}=1.5 \times 10^{-9} \ldots 103$

$4.4 \quad \Sigma_{\ell}$ benchmark at $m_{\ell}=400 \mathrm{GeV}, x=0.025, \lambda=5 \times 10^{-12}, \lambda=2.5 \times 10^{-9} \ldots 108$

$4.5 \Sigma_{\ell}$ benchmark at $m_{\ell}=800 \mathrm{GeV}, x=10^{-6}, \lambda=8 \times 10^{-10}, \lambda=7.8 \times 10^{-10} \quad \ldots 108$ 



\section{Abbreviations}

$\begin{array}{ll}\text { LHC } & \text { Large HadronCollider } \\ \text { ID } & \text { Indirect Detection } \\ \text { (N)LO } & \text { (Next) Leading Order } \\ \text { HSCP } & \text { Heavy Stable Charged Particle } \\ \text { RPC } & \text { R- Parity Conserving } \\ \text { RPV } & \text { R- Parity Violating } \\ \text { SM } & \text { Standard Model (of particle physics) } \\ \text { MSSM } & \text { Minimal Supersymmetric Standard Model } \\ \text { CMB } & \text { Cosmic Microwave Background } \\ \text { BBN } & \text { Big Bang Nucleosynthesis } \\ \text { CMS } & \text { Compact Muon Solenoid } \\ \text { DM } & \text { Dark Matter } \\ \text { (C,W,H)DM } & \text { (Cold,Warm,Hot) Dark Matter } \\ \text { MG } & \text { MadGraph } \\ \text { IP } & \text { Interaction Point } \\ \text { GR } & \text { General Relativity } \\ \Lambda \text { CDM } & \text { standard model of cosmology } \\ \text { LSS } & \text { Large Scale Structure } \\ \text { BC } & \text { Bullet Cluster } \\ \text { QFT } & \text { Quantum Field Theory } \\ \text { UV } & \text { Ultra Viole } \\ \text { GUT } & \text { Grand Unified Theory } \\ \text { VEV } & \text { Vacuum Expectation Value } \\ \text { FRW } & \end{array}$



To my grandmother Alcide... 



\section{Introduction}

During the last 80 years, a new paradigm has emerged in our understanding of the Universe, according to which matter visible by telescopes represents a small fraction of the total amount of matter present in the Universe. Most of the matter instead appears to be "dark", in the sense that does not emit nor absorb light at any significant level. This new type of matter went under the name of Dark Matter (DM).

The existence of this large amount of non-luminous, non-baryonic DM is at the present time well-established through several observations made on galactic up to cosmological length-scale. Thus, nowadays DM is thought to account for more than $80 \%$ of the total matter density of the Universe and be present in form of large massive halos in galaxies.

Although today we have many evidences for DM, its nature is still one of the most unsolved but exciting mysteries in cosmology. In order to unravel this mystery, in the past much effort is being made to understand whether DM could be accounted for by some combination of baryonic matter, or whether it required particles beyond the Standard Model particle spectrum. By now, it is well established that the DM nature requires physics beyond the Standard Model (SM).

In absence of a fundamental motivation according to which DM must be stable, DM can also decay as long as its lifetime is much longer that the age of the Universe. Therefore, it is very important to investigate the potential signatures arising from these decays. In the DM decay paradigm, the DM particle can also interact as single state with charged SM and non-SM states since there is no symmetry to stabilize it. Secondly, DM must interact with suppressed couplings to any sector, and therefore it can be produced via Freeze-in and SuperWIMP mechanisms, based on smaller couplings than those of the WIMP mechanism (based on the electroweak scale). Remarkably important to detect unstable DM at colliders is to investigate the phenomenology of models containing DM along with other particles which in turn have clear expected signatures. It is indeed thought that this super weak DM could be discovered or even excluded only by studying globally the phenomenology of such models. Motivated by this comment, in this thesis we focus on two simple decaying DM models that can be disentangled along with their couplings by combining the decay of DM with the signals at collider.

Until now, DM has only been observed via gravitational interactions. Thus, in principle a DM candidate interacting only with gravity is even possible. Supersymmetry (SUSY) offers a candidate of this type, i.e. gravitino. The gravitino shows up as a natural candidate for DM in local SUSY, i.e. Supergravity (SUGRA), according to which, it must be very weakly interacting, since, as part of the gravity supermultiplet, all its couplings are suppressed either by the Planck scale (for the 3/2-spin component) or by the SUSY breaking scale (for the $1 / 2$-spin component). Despite these very suppressed couplings, the gravitino can be generated, on one hand, via thermal production in accordance with the observed DM density and wih baryogenesis via thermal leptogenesis if a high reheating temperature after inflation occurred, on the other hand, via the decay of the NLSP out of equilibrium depending on the SuperWIMP mechanism. 
At the present time a strong effort is undertaken to search for signatures of particle DM candidates. In fact, according to the model, signatures are still expected from proton-proton collisions at LHC (collider detection), scattering DM particle-nuclei in underground detectors (direct detection), and exotic contributions from the annihilation or the decay of DM particles in the galactic halo to the spectra of cosmic rays (indirect detection). Only a combination of evidences from all of these detection strategies, indeed, allows to connect the DM cosmological observation with a particle physics explanation

After the first run of LHC, no signal of new physics has been found in terms of the DMmotivated channels containing missing energy. Even though the next LHC run could still detect a WIMP-like signal, especially from the electroweakly charged sector, we investigate in this thesis the phenomenology of two models, beyond the SM of particle physics, for unstable DM which are expected to show up as cosmological consistent signals at colliders. These signals are represented by long-lived particles, metastable particles and, finally, prompt particles while their cosmological consistency lies in the requirements that DM is in agreement with the indirect detection constraints, the value of the present-day DM and, at last, with baryonic asymmetry via thermal leptogenesis (only for gravitino).

Firstly we discuss a gravitino DM scenario both with and without R-parity and their phenomenology when the NLSP is the lightest stop, produced in the proton-proton collision at LHC, and the rest of the supersymmetric particles were outside the LHC reach. The stop NLSP is well-motivated since the stop is expected to be firstly the lightest colored state, secondly not too heavy and thirdly not too constrained by LHC with respect the other colored states. Furthermore, the stop NLSP is also expected to be long-lived and so give rise either to displaced vertices or metastable tracks. Since in this scenario the LSP gravitino, due to its extremely weak interactions, makes the stop NLSP long-lived, the latter can decay during or after the BBN, thus spoiling its predictions. Therefore, besides the constraint from the right DM abundance, we also have to take into account the constraints from BBN. The LHC production of stop and their following exponential decay is simulated by MadGraph 5 and Python respectively. The goal is to determine the LHC reach for direct NLSP stop production, regardless of the stop decay channel, and compare the parameter region with the cosmologically viable one. Specifically, we study stop displaced vertices inside Pixel and Tracker, the two closest inner parts of CMS to the interaction point. Finally, we discuss the possibility of distinguishing the RPC and RPV stop decays if they occur inside the two CMS studied parts, specifically if at least one charged lepton is produced in the decay. In the RPV scenario, the gravitino decay can lead to a diffuse $\gamma$-ray flux that, if it is compared with that one observed by the indirect detection searches, can strongly constrain the RP breaking parameter.

Secondly, we investigate a minimal scenario of decaying DM, featuring a DM Majorana fermion SM singlet and a scalar charged under the SM gauge group. The scalar is coupled to the DM Majorana fermion and a chiral SM fermion via the coupling $\lambda$ and to only chiral SM fermions via the coupling $\lambda^{\prime}$. Through these two couplings, DM can be produced by SuperWIMP and Freeze-in mechanisms and DM three-body decays can easily occur, and so constrain the DM lifetime via the current indirect detection searches of anti-proton and positron. Due to this DM long lifetime, requiring the new scalar field to be within the LHC reach constrains all couplings to be suppressed and therefore points also to regions of (near)-collider-metastability for these exotic states. It is therefore natural to look for such particles at the LHC and CLIC in the presence of displaced vertices or metastable tracks. The LHC and CLIC productions of the scalar and their following exponential decay are simulated by MadGraph 5 and Python, respectively. The 
goal is to individuate the parameter space where the model is both cosmologically viable and observable through multiple signals and investigate the sentivity of LHC and CLIC in the near future. It is noteworthy that this minimal model can be embedded in more complex models, like supersymmetry with R-parity violation, but its main phenomenological characteristics are independent from the particle physics framework, at least concerning the DM phenomenology.

Lastly, we exploit the previous very simple and rather predictive decaying DM scenario to study whether a similar interplay between collider and DM indirect detection can be established for DM masses at the $\mathrm{keV}$ scale, for which only two-body, one-loop induced, decays into a neutrino and a photon are possible, thus reproducing in the model the $3.55 \mathrm{keV} \mathrm{X}$-ray line signal. This photon line has been recently detected in the combined spectrum of a large set of $\mathrm{X}$-ray galaxy clusters as well as in the combined observation of the Perseus Cluster and the M31 galaxy. In this scenario the signal of ID allows to match the DM lifetime with the observed one. Moreover, the requirement that DM is out of the equilibrium in the early allows Freeze-in to operate and produce DM. At last we also discuss two extensions/modifications of this scenario, allowing for further couplings and fields. In the first case we have DM decays into a photon and a new SM singlet, rather than the neutrino, which can contribute to the number of light species $N_{e f f}$, probed by CMB experiments. In the second case we have a DM sterile neutrino, produced via Freeze-in mechanism. Although the scalar field is not responsible for the DM radiative decay, it is necessary for the DM production. 



\title{
Chapter 1
}

\section{Models for Dark Matter particles and CMS detector}

\author{
"Supersymmetry is so beautiful and suggestive that most of us think it \\ has got to show up some time in nature, although so far it hasn't"
}

Steven Weinberg

Over the last few decades the physics community has been developing new theories beyond the Standard Model (SM) of particle physics, which has been tested successfully to a few per mil at the LEP collider, in order to explain and discuss what SM has left aside, such as diversity of mass scales, vacuum energy, neutrino masses, evidence for Dark Matter etc.

In this chapter we introduce two models for Dark Matter (DM) particles. The first one is the well-known Minimal Supersymmetric Standard Model (MSSM) with gravitino DM and Lightest Supersymmetric Particle (LSP). The second one is a minimal model of DM decaying where DM is a neutral Majorana fermion. The latter model is minimal in the sense that besides DM only one extra particle (scalar non-trivially charged under at least one part of the SM gauge group) has been added to the SM particle spectrum.

To this end, we start presenting a brief introduction to Supersymmetry, one of the most popular theories beyond the SM that also provides with some DM candidates (e.g. neutralino). Quite precisely, this introduction is neither a review article, nor a summary of Supersymmetry. In fact, there are already so many excellent reviews available on-line that we are spoilt for choice. Among them the standard reference for a comprehensive introduction and review of Supersymmetry has been written by Stephen Martin [1]. Later we promote Supersymmetry to a local symmetry so as to include gravity in the model and therefore the so-called gravitino, the superpartner of the spin-2 graviton and a good DM candidate as well. Such a model is known as 
Supergravity. A very good and useful review about it has been written by Bernard de Wit [2]. Although the two above-cited references are the most used by students, they are not by no means exhaustive, indeed other introductions to these subjects with applications to particle physics have also been written recently by Peskin [3], Olive [4], Drees [5], Binétruy [6] and, finally, Wess and Bagger in the very famous book entitled "Supersymmetry and Supergravity" [7]. We conclude this chapter by studying the above-mentioned minimal model of DM decaying, previously introduced in [8], and giving a brief description of LHC experiment and CMS detector.

The aim of this chapter is to equip the reader with the necessary theoretical tools to understand and process more easily the search of the coming chapters. Before going into detail, we remark that although LHC has discovered in 2012 the missing building block of SM, namely the Higgs boson $[9,10]$, so far it has found no direct evidence of new physics beyond this model.

\subsection{Basics of Supersymmetry}

Supersymmetry (SUSY) is a space-time symmetry relating particles of integer spin (bosons) and particles of half integer spin (fermions). The mapping of bosons into fermions and viceversa is obtained by introducing new fermionic generators $Q_{\alpha}$ as follows:

$$
\left.\left.\left.\left.Q_{\alpha} \mid \text { boson }\right\rangle \simeq \mid \text { fermion }\right\rangle_{\alpha}, \quad Q_{\alpha} \mid \text { fermion }\right\rangle^{\alpha} \simeq \mid \text { boson }\right\rangle,
$$

where $\alpha$ must be a spinor label.

The operators $Q_{\alpha}$, which are chosen by convention to be Majorana spinors, must satisfy nontrivial anti-commutation relations. In particular, if we allow for only one set of these fermionic generators $(N=1$ Supersymmetry), the anti-commutation relations read:

$$
\left\{Q_{\alpha}, Q_{\dot{\beta}}^{\dagger}\right\}=2\left(\sigma^{\rho}\right)_{\alpha \dot{\beta}} P_{\rho},
$$

where $P_{\rho}$ represents the four-momentum generator and $\sigma^{\rho}$ are the Pauli matrices, defined as:

$$
\sigma^{1}=\left(\begin{array}{cc}
0 & 1 \\
1 & 0
\end{array}\right), \quad \sigma^{2}=\left(\begin{array}{cc}
0 & -i \\
i & 0
\end{array}\right), \quad \sigma^{3}=\left(\begin{array}{cc}
1 & 0 \\
0 & -1
\end{array}\right) .
$$

These transformations along with the following commutation and anticommutation relations:

$$
\begin{aligned}
& {\left[M^{\rho \sigma}, Q_{\alpha}\right]=-i\left(\sigma^{\rho \sigma}\right)_{\alpha}^{\beta} Q_{\beta},} \\
& {\left[Q_{\alpha}, P^{\rho}\right]=0,} \\
& \left\{Q_{\alpha}, Q_{\beta}\right\}=\left\{Q_{\alpha}^{\dagger}, Q_{\beta}^{\dagger}\right\}=0,
\end{aligned}
$$

form the so-called $N=1$ Super-Poincaré algebra. The operator $M^{\rho \sigma}$ indicates the angular momentum generator while the matrix $\sigma^{\rho \sigma}$ is identified as: $\sigma^{\rho \sigma}=\frac{i}{2}\left[\gamma^{\rho}, \gamma^{\sigma}\right]$. Particularly, the above anti-commutation relations between $Q$ and $Q^{\dagger}$ show the connection of Supersymmetry with the translations and space-time diffeomorphisms which leads directly to gravity in the case of promotion of Supersymmetry from a global to a local symmetry. The extension of such an algebra to more sets of fermionic operators is trivial and leads to more complicated Supersymmetry models. However, it has been found that $N>1$ theories are ruled out as a low-energy extension of SM (i.e. TeV-limit). 
Supersymmetry is the unique non-trivial extension of the Poincare group, the symmetry group that lies at the heart of every Quantum Field Theory (QFT) consisting of Lorentz transformations (LT) and translations:

$$
\begin{aligned}
& {\left[P^{\rho}, P^{\sigma}\right]=0} \\
& {\left[P^{\rho}, M^{\nu \sigma}\right]=i\left(g^{\rho \nu} P^{\sigma}-g^{\rho \sigma} P^{\nu}\right),} \\
& {\left[M^{\mu \nu}, M^{\rho \sigma}\right]=-i\left(g^{\mu \rho} M^{\nu \sigma}+g^{\nu \sigma} M^{\mu \rho}-g^{\mu \sigma} M^{\nu \rho}-g^{\nu \rho} M^{\mu \sigma}\right) .}
\end{aligned}
$$

Indeed, in 1975 Haag, Lopuszanski and Sohnius showed in [11] that the Coleman-Mandula no-go theorem [12], which states that any symmetry compatible with an interacting relativistic QFT is a direct product of the Poincaré algebra with an internal symmetry $G$, i.e.

$$
\left[G, P_{\rho}\right]=\left[G, M_{\rho \mu}\right]=0
$$

could be evaded by weakening the assumption that the symmetry algebra only involved commutators, and so bosonic generators. Allowing for fermionic generators and their anti-commutation relations, it eventually turned out that the set of allowed symmetries was really enlarged.

The main feature of Supersymmetry models is that each of the known fundamental particles is in either a gauge supermultiplet (consisting of a gauge field and a gaugino fermion) or chiral supermultiplet (consisting of a Weyl fermion and a complex scalar) and must correspond to a superpartner with spin differing by $1 / 2$ unit. In Table 1.1 we can see such a correspondence for the matter fields (quarks and leptons), the gauge fields (gauge bosons) and the Higgs field. In other words, every supermultiplet consists of states of the same representation under the SM gauge group but different representation under the Lorentz group. The chiral multiplets contain the matter fermions or scalars and their superpartners whereas the gauge multiplets contain the gauge bosons and their superpartners, the gauginos, one for each SM group generator and gauge coupling $g$. We provide more detail on these two supermultiplets later in this section.

If Supersymmetry were realized in Nature as an exact symmetry, the particles so related should have almost all their characteristics, such as mass and charge, preserved. This is apparently a disaster for the idea of Supersymmetry since it predicts that the new supersymmetric particles should be easy to detect through many experiments. The crucial caveat to this negative result is the condition that supersymmetry be realized as an exact symmetry. If it were spontaneously broken, instead, we would obtain a difference in masses of the particles related by the symmetry (at least at energies of order $10^{2} \mathrm{GeV}$ or lower), which could explain the lack of detection of the heavier supersymmetric particles in the running experiments. The idea is then that Supersymmetry is broken at some scale $M_{s}$, such that at energies $E>M_{s}$ the theory behaves in a supersymmetric way, while at energies $E<M_{s}$ it does not.

Although not yet confirmed experimentally, there are many motivations why this new symmetry might interest physicists. The first one is represented by the hierarchy problem of SM,

\begin{tabular}{cc}
\hline SM particles & SUSY partners \\
\hline gauge bosons & gauginos \\
quarks, leptons & scalars \\
Higgs & higgsino \\
\hline
\end{tabular}

TABLE 1.1: SM/SUSY particle spectrum. 


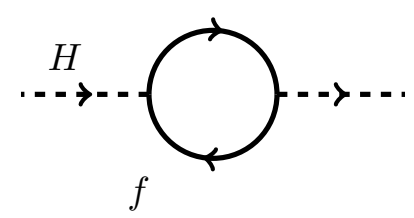

FiguRE 1.1: One-loop radiative correction to the Higgs mass due to the fermion couplings.

that is the large discrepancy between aspects of the weak force and gravity, or in terms of energy scales, the big difference between the electroweak scale $(O(100) \mathrm{GeV})$ and the reduced Planck scale:

$$
M_{P l}=\frac{1}{\sqrt{8 \pi G_{N}}} \simeq 2.4 \times 10^{18} \mathrm{GeV}
$$

which is the physical scale where gravity becomes comparable with the other known forces and thus it can not be neglected in elementary particle interactions. This problem arises from the quadratic radiative corrections to the Higgs mass $m_{H}$ given by the Dirac fermion one-loop of Figure 1.1 and expressed analytically by the formula:

$$
\Delta m_{H}^{2} \sim-\lambda_{f}^{2} \Lambda^{2}
$$

The terms $\lambda_{f}$ and $\Lambda$ are, respectively, the coupling between Higgs $H$ and the fermion $f$ and the Ultra-Violet (UV) cut-off. Notice that the latter should be naturally around the TeV scale and, thus, SM should be seen as an effective theory valid only at $E<M_{\mathrm{eff}} \sim \mathrm{TeV}$ in order to protect the Higgs mass at its physical value of $125 \mathrm{GeV}$ without taking into consideration SUSY.

Such quadratic divergences, on the other hand, can be eliminated in Supersymmetry theories by means of the contribution of the bosonic superpartners to the radiative corrections, namely with no fine-tuning. In fact, the couplings between Higgs $H$ and a scalar $S$ provide one-loop radiative contributions to the Higgs mass which are opposite in sign with respect to those arising from the fermion couplings given by the scalar one-loops of Figure 1.2 and the formula:

$$
\Delta m_{H}^{2} \sim \lambda_{S} \Lambda^{2} \quad \text { with } \quad \lambda_{S}=-\lambda_{f}^{2} .
$$

The second motivation to believe in Supersymmetry is represented by the unification of the three gauge couplings: $\alpha_{\alpha}=g_{\alpha}^{2} / 4 \pi$, with $\alpha=1,2,3$. In particular, $g_{1}=\sqrt{5 / 3} g^{\prime}, g_{2}=g$ and $g_{3}=g_{s}$ denote, in the given order, the two electroweak coupling constants and the strong coupling constant of the unbroken SM gauge group $S U(3)_{c} \times S U(2)_{L} \times U(1)_{Y}$. Actually, $g_{1}, g_{2}$ and $g_{3}$ are not constants in the true sense of the word, they run indeed according to the Renormalization Group (RG) equations which depend on the particle content of the theory and

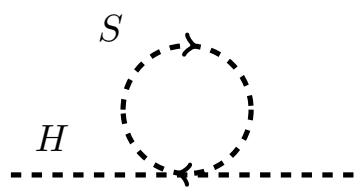

Figure 1.2: One-loop radiative correction to the Higgs mass due to the scalar couplings. 
eventually determine their energy evolution. If we do not allow for new particles besides the SM ones to exist at a much higher energy scale than the electroweak scale, the three gauge constant couplings only approximately meet thus giving an unnatural fremework from a theoretical point of view. On the contrary, with the Supersymmetry particle content at the $\mathrm{TeV}$ scale, the gauge couplings exactly unify at the unification scale $M_{G U T} \sim 10^{15} \mathrm{GeV}$, called Grand Unified Theory (GUT) scale as we can see in Figure 1.3.

The third motivation is that SUSY provides a valuable and very promising Dark Matter (DM) candidate: The Lightest Supersymmetric Particle (LSP) of its particle spectrum. Indeed, the neutrino particle, the only SM candidate for DM, can only set up a small fraction of DM because of the limits from the large-scale structure and the high-redshift galaxies. In order to explain the total fraction of DM in the Universe $(\sim 22 \%$ of the total energy density of the Universe) from a particle point of view, a beyond SM Dark matter candidate is necessary.

Even though we discuss R-parity symmetry only at the end of this section, now we can reveal in advance that the LSP is absolutely stable and so a very natural DM candidate in a R-parity conserving scenario, namely if the R-parity symmetry is preserved in the theory. On the contrary, in a R-parity violating scenario the LSP can decay but nevertheless it can remain a viable DM candidate under certain conditions that we examine later. For the sake of completeness, let us also disclose that in order to constitute DM the LSP must be colorless and electromagnetically neutral. Thus, it is thought to interact only with gravity and the electroweak force and in case with itself. We will come back to this interesting point in the next chapter.

\subsection{Minimal Supersymmetric Standard Model}

The simplest and most economic supersymmetric model is the minimal supersymmetric extension of SM of particle physics (MSSM, for Minimal Supersymmetric Standard Model) which introduces only the couplings and fields that are indispensable for the consistency of the theory. As a matter of fact, the lagrangian we construct should not only be gauge invariant under

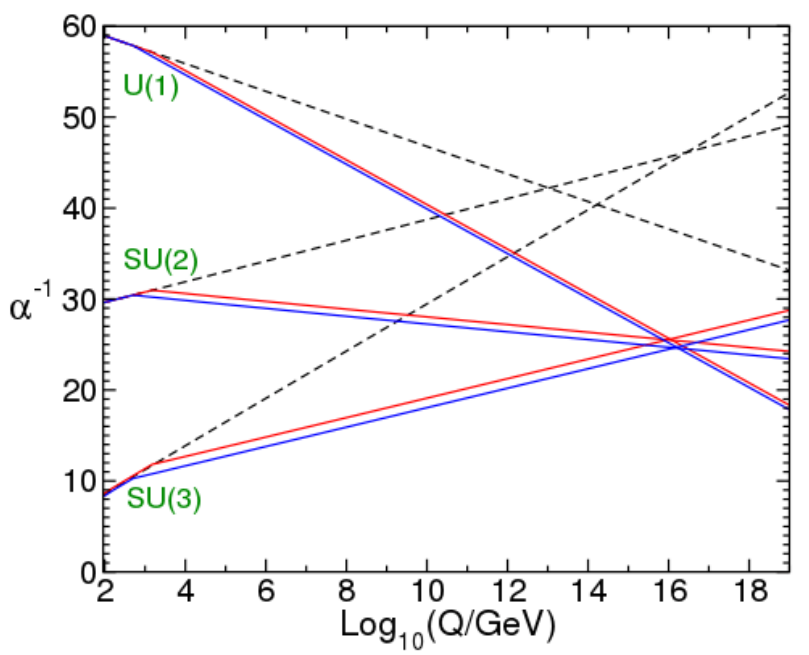

FIGURE 1.3: Two-loop renormalization group evolution of the inverse gauge couplings $\alpha_{\alpha}^{-1}(Q)$ in the Standard Model (dashed lines) and the MSSM (solid lines). 
the Standard Model gauge group but also now be supersymmetric invariant. Furthermore, the MSSM is renormalisable and anomaly free, just like the Standard Model is.

Before we proceed to introduce the particle spectrum, let us remind ourselves that ordinary quantum fields are upgraded in supersymmetric theories to so-called supermultiplets or superfields, depending on the book/review. Specifically, supermultiplets are multiplets which collect fermion-boson pairs which transform in to each other.

We will deal with two kinds of supermultiplets: Vector (or gauge) supermultiplets and chiral supermultiplets. A chiral supermultiplet contains a weyl fermion $\chi$, a complex scalar $\phi$ and an auxiliary scalar field generally denoted by F. A vector supermultiplet contains a spin 1 vector boson $A_{\mu}^{a}$, a spin 1/2 Majorana fermion $\lambda^{a}$ and an auxiliary scalar field called $D^{a}$.

\section{Partners and Superpartners}

The minimal supersymmetric extension of the SM is built by replacing each standard model matter field by a chiral supermultiplet and each vector field by a vector supermultiplet. In this way the existing particle spectrum of the SM is doubled.

The particle spectrum of the MSSM and their transformation properties under the SM gauge group $S U(3)_{c} \times S U(2)_{L} \times U(1)_{Y}$ is given by Table 1.2 and 1.3. In particular, Table 1.2 shows the particle content of the gauge supermultiplets, which consists of the electroweak gauge bosons $\left(W_{\mu}^{a}, B_{\mu}\right)$ and the corresponding fermionic gauginos (winos $\tilde{W}^{a}$, binos $\tilde{B}$ ) as well as the color gauge bosons (gluons $G_{\mu}^{a}$ ) and the corresponding fermionic parts (gluinos $\tilde{g}^{a}$ ). Table 1.3 shows instead the particle content of the chiral supermultiplets, which consists of three generations of left-handed and right-handed leptons $\left(L^{i}, E^{c i}\right)$ and quarks $\left(Q_{h}^{i}, U_{h}^{c i}, D_{h}^{c i}\right)$, the corresponding scalar sleptons $\left(\tilde{L}^{i}, \tilde{E}^{* i}\right)$ and squarks $\left(\tilde{Q}_{h}^{i}, \tilde{U}_{h}^{* i}, D_{h}^{* i}\right)$, and all their corresponding anti-particles. Here, the index $i(=1,2,3)$ stands for the generator index of (s)leptons and (s)quarks whereas $h(=r, g, b)$ represents the color index of (s)quarks. In addition, in these supermultiplets the two Higgs-doublets $\left(H_{d}, H_{u}\right)$ are located along with the corresponding fermionic supersymmetric partners (down-type higgsino $\tilde{H}_{d}$, up-type higgsinos $\tilde{H}_{u}$ ) and all their corresponding anti-particles.

The most important feature in the MSSM spectrum is represented by the requirement of at least two Higgs multiplets. More precisely, this doubling of the Higgs sector with respect to the SM case is necessary basically for two reasons: the gauge anomaly cancellation and the impossibility of giving mass to the up quarks through one Higgs doublet only. Regarding the former reason, the higgsinos are fermions in the fundamental representation of $S U(2)_{L}$, hence two of them are needed, with opposite hypercharge, not to spoil the anomaly-free properties of SM. Regarding the latter reason, instead, a second independent Higgs doublet in the conjugate representation is essential because the charge conjugate of the Higgs field can not enter in a SUSY potential, being it only a function of $H$. In fact, while one Higgs supermultiplet gives

\begin{tabular}{lccc}
\hline Name & Gauge bosons & Gauginos & $\left(S U(3)_{c}, S U(2)_{L}, U(1)_{Y}\right)$ \\
\hline$B$ boson, bino & $A_{\mu}^{(1)}=B_{\mu}$ & $\lambda^{(1)}=\tilde{B}$ & $(\mathbf{1}, \mathbf{1}, \mathbf{0})$ \\
$W$ bosons, winos & $A_{\mu}^{(2) a}=W_{\mu}^{a}$ & $\lambda^{(2) a}=\tilde{W}^{a}$ & $(\mathbf{1}, \mathbf{3}, \mathbf{0})$ \\
gluons, gluinos & $A_{\mu}^{(3) a}=G_{\mu}^{a}$ & $\lambda^{(3) a}=\tilde{g}^{a}$ & $(\mathbf{8}, \mathbf{1}, \mathbf{0})$ \\
\hline
\end{tabular}

TABLE 1.2: Gauge supermultiplets of the MSSM. 


\begin{tabular}{lccc}
\hline Name & Scalars $\phi^{i}$ & Fermions $\chi_{L}^{i}$ & $\left(S U(3)_{c}, S U(2)_{L}, U(1)_{Y}\right)$ \\
\hline Sleptons, leptons & $\tilde{L}^{i}=\left(\begin{array}{c}\tilde{\nu}_{L}^{i} \\
\tilde{e}_{L}^{L}\end{array}\right)$ & $L^{i}=\left(\begin{array}{c}\nu_{L}^{i} \\
e_{L}^{-i}\end{array}\right)$ & $\left(\mathbf{1}, \mathbf{2},-\frac{\mathbf{1}}{\mathbf{2}}\right)$ \\
& $\tilde{E}^{* i}=\tilde{e}_{R}^{-* i}$ & $E^{c i}=e_{R}^{-c i}$ & $(\mathbf{1}, \mathbf{1}, \mathbf{1})$ \\
Squarks, quarks & $\tilde{Q}_{h}^{i}=\left(\begin{array}{c}\tilde{u}_{L, h}^{i} \\
\tilde{d}_{L, h}^{i}\end{array}\right)$ & $Q_{h}^{i}=\left(\begin{array}{c}u_{L, h}^{i} \\
d_{L, h}^{i}\end{array}\right)$ & $(\mathbf{3}, \mathbf{2}, \mathbf{1}$ \\
& $\tilde{U}_{h}^{* i}=\tilde{u}_{R, h}^{* i}$ & $U_{h}^{c i}=u_{R, h}^{c i}$ & $\left(\overline{\mathbf{3}}, \mathbf{1},-\frac{\mathbf{2}}{\mathbf{3}}\right)$ \\
& $\tilde{D}_{h}^{* i}=\tilde{d}_{R, h}^{* i}$ & $D_{h}^{c i}=d_{R, h}^{c i}$ & $(\overline{\mathbf{3}}, \mathbf{1}, \mathbf{1}$ \\
Higgs, higgsinos & $H_{d}=\left(\begin{array}{c}H_{d}^{0} \\
H_{d}^{-}\end{array}\right)$ & $\tilde{H}_{d}=\left(\begin{array}{c}\tilde{H}_{d}^{0} \\
\tilde{H}_{d}^{-}\end{array}\right)$ & $\left(\mathbf{1}, \mathbf{2},-\frac{\mathbf{1}}{\mathbf{2}}\right)$ \\
& $H_{u}=\left(\begin{array}{c}H_{u}^{+} \\
H_{u}^{0}\end{array}\right)$ & $\tilde{H}_{u}=\left(\begin{array}{c}\tilde{H}_{u}^{+} \\
\tilde{H}_{u}^{0}\end{array}\right)$ & $\left(\mathbf{1}, \mathbf{2}, \frac{\mathbf{1}}{\mathbf{2}}\right)$ \\
\hline
\end{tabular}

TABLE 1.3: Chiral supermultiplets of the MSSM.

masses to the up-type quarks, the other gives masses to the down type quarks and charged leptons.

\section{Superpotential}

The MSSM is defined by the above minimal field content as well as the minimal superpotential necessary to account for the necessary Yukawa couplings and mass terms, namely:

$$
W=\eta_{a b}\left(y_{e} H_{d} L^{a} E^{c b}+y_{d} H_{d} Q^{a} D^{c b}-y_{u} H_{u} Q^{a} U^{c b}\right)+W_{\mu}
$$

where

$$
W_{\mu}=\eta_{a b} \mu H_{u}^{a} H_{d}^{b}
$$

A sum over $S U(2)_{L}$ generation indices $a, b(=1,2,3)$ and the suppressed gauge indices is implied. The whole superpotential is written in a gauge-invariant way because of the use of the tensor $\eta_{a b}$ that ties together the two $S U(2)_{L}$ generation indices. Such a superpotential also conserves the R-parity symmetry, discrete symmetry that we introduce later in this section. The parameter $\mu$ appearing in the " $\mu$ term", as the term $W_{\mu}$ is usually called, denotes the supersymmetric version of the Higgs boson mass in the SM. Thus, the superpotential represents a supersymmetrization of the Standard Yukawa couplings plus a bilinear Higgs term.

In Eq.(1.9), the dimensionless Yukawa couplings $y_{u}, y_{d}, y_{e}$ are all $3 \times 3$ matrices in generation space, with no generation indices for the Higgs multiplets. These matrices determine the current masses and CKM mixing angles of the ordinary quarks and leptons, after the neutral scalar components of $H_{u}$ and $H_{d}$ get the Vacuum Expectation Values (VEVs). Since the top quark, bottom quark and tau lepton are the heaviest fermions in the Standard Model, it is often useful to make an approximation that only the $(3,3)$ generation components of each of the Yukawa couplings are important. In this limit, only the third family and Higgs fields contribute to the MSSM superpotential. 
The superpotential enters the lagrangian of the theory through the terms:

$$
\mathcal{L}_{S U S Y}=-\frac{1}{2}\left(W^{i j} \chi_{i} \chi_{j}+W_{i j}^{*} \chi^{i^{\dagger}} \chi^{j^{\dagger}}\right)-W^{i} W_{i}^{*}
$$

where $W^{i}=\partial W / \partial \phi_{i}, W_{i}^{*}=\partial W / \partial \phi^{i^{*}}$ and $W^{i j}=\partial^{2} W / \partial \phi_{i} \partial \phi_{j}$. Let us remind that $\phi_{i}$ and $\chi_{i}$ are the complex scalar field and the left-handed two component Weyl fermion, in the given order, of the chiral supermultiplets.

\section{Supersymmetry Breaking}

As already mentioned in Section 1.1, Supersymmetry must be broken since no supersymmetric particles have been detected so far. A Supersymmetry breaking mechanism is therefore necessary in order to shift all mass terms of the supersymmetric particles to higher energies, preferably in $\mathrm{TeV}$ range, so as to hide these new particles from the current experiments. Additionally, to solve the hierarchy problem, the Supersymmetry breaking must be soft, i.e. the Supersymmetry breaking parameters must not introduce quadratic divergences.

Since SUSY cannot be spontaneously broken in any renormalisable extension of the MSSM (otherwise we would have squarks with masses less or equal to the masses of either the up- or down-quarks, as found by Georgi and Dimopoulos [13]), one commonly assumes the existence of a hidden sector, consisting of particle that are neutral with respect to the SM gauge groups, and a visible sector, containing instead the MSSM particles. The SUSY breaking is thought to happen in the hidden sector, when one of the hidden sector fields obtains a non-vanishing F-term VEV $(\langle F\rangle)$, and to be mediated to the MSSM through some mechanism. Such a F-term of the superpotential is given by:

$$
\mathrm{F} \text {-term }=W_{i} F^{i}+\text { h.c. }
$$

where $F^{i}$ stands for the auxiliary field of the chiral supermultiplet while $W_{i}$ is defined above. In this case we commonly speak of soft SUSY breaking.

The soft SUSY breaking decouples the origin of supersymmetry breaking from its phenomenological consequences. In effect, soft SUSY breaking adds explicit terms to the supersymmetric SM Lagrangian to break the symmetry, namely it makes sure that the Lagrangian contains terms which do not preserve supersymmetry by themselves. In order for them not to ruin the nice and welcome UV properties of supersymmetric theories, these terms should have positive mass dimension, in other words they should be irrelevant in the far UV. In such scenario, the energy scale where supersymmetry is broken enters explicitly in the Lagrangian.

As the generators of Supersymmetry are fermionic generators, the SUSY breaking produces a massless goldstone fermion (goldstino), which plays the role similar to the massless goldstone scalar boson of the global electroweak symmetry breaking. Similarly to the electroweak case, we again deal with a problem of global SUSY since no massless fermion has been detected so far.

In MSSM, there are about 105 new soft SUSY breaking parameters. All of them influence the low-energy physics but, as above-mentioned, preserve the cancellation of the divergences for the Higgs mass (i.e. there is no hierarchy problem). Among all these parameters, there are: the masses $M_{1}, M_{2}$ and $M_{3}$ of the electroweak and strong gauginos, respectively, the soft masses of the squarks and sleptons and, finally, the soft masses $m_{H_{u}}^{2}$ and $m_{H_{d}}^{2}$ of the Higgs doublets. 
Furthermore, in case of broken R-parity, numerous additional soft terms come up, among which the Higgs-slepton mixing parameters $B_{i}$ and $m_{L_{i} H_{d}}^{2}$.

For the sake of completeness, we point out here that the hidden sector F-term also generates the gravitino mass, as explained later in Section 1.3.

\section{Electroweak Symmetry Breaking}

As is common knowledge in the SM theory, the electroweak symmetry is spontaneously broken down to electromagnetism, i.e. in terms of gauge groups:

$$
S U(2)_{L} \times U(1)_{Y} \longrightarrow U(1)_{e m}
$$

In the Supersymmetric extensions such a phase transition may be achieved dynamically via the radiative corrections to the soft masses $m_{H_{u}}$ and $m_{H_{d}}$ of the two complex Higgs doublets. The neutral Higgs fields then acquire the vacuum expectation values $\left\langle H_{u}^{0}\right\rangle=v_{u}$ and $\left\langle H_{d}^{0}\right\rangle=v_{d}$, which are connected to the SM Higgs VEV $v \simeq 174 \mathrm{GeV}$ as follows:

$$
\begin{aligned}
& v^{2}=v_{u}^{2}+v_{d}^{2}, \\
& v_{u}=v \sin \beta, \\
& v_{d}=v \cos \beta,
\end{aligned}
$$

Thus, the ratio between the two complex Higgs doublet VEVs is commonly referred to as:

$$
\tan \beta=\frac{v_{u}}{v_{d}}
$$

According to electroweak symmetry breaking via the Higgs mechanism, the electroweak gauge bosons absorb the three massless degrees of freedom of the two complex Higgs doublets, namely the Goldstone bosons $G^{0}, G^{+}$and $G^{-}$. These become thus the longitudinal modes of the massive $Z^{0}$ and $W^{ \pm}$vector bosons. Such a mechanism is the so-called Higgs mechanism of the electroweak symmetry breaking.

The new mass eigenstates after electroweak symmetry breaking are the electrically neutral photon $A$ and the electrically neutral $Z$ boson, defined by the rotation:

$$
\left(\begin{array}{c}
A_{\mu} \\
Z_{\mu}^{0}
\end{array}\right)=\left(\begin{array}{cc}
\cos \theta_{W} & \sin \theta_{W} \\
-\sin \theta_{W} & \cos \theta_{W}
\end{array}\right)\left(\begin{array}{c}
B_{\mu} \\
W_{\mu}^{0}
\end{array}\right),
$$

as well the electrically charged $W^{ \pm}$bosons, defined as:

$$
W_{\mu}^{ \pm}=\frac{1}{\sqrt{2}}\left(W_{\mu}^{1} \mp i W_{\mu}^{2}\right) .
$$

The angle $\theta_{W}$ is called weak mixing angle (or simply Weinberg angle) and can be expressed in terms of the $S U(2)_{L}$ gauge coupling $g$ and the $U(1)_{Y}$ gauge coupling $g^{\prime}$, as follows:

$$
\begin{aligned}
\sin \theta_{W} & =\frac{g^{\prime}}{\sqrt{g^{2}+g^{\prime 2}}}, \\
\cos \theta_{W} & =\frac{g}{\sqrt{g^{2}+g^{\prime 2}}} .
\end{aligned}
$$


The elementary charge $e$, which is the gauge coupling of the residual symmetry group $U(1)_{e m}$, is linked to the two couplings $g, g^{\prime}$ and the mixing angle $\theta_{W}$ through the equality:

$$
e=\sqrt{4 \pi \alpha}=g \sin \theta_{W}=g^{\prime} \cos \theta_{W} .
$$

After the Higgs mechanism, the photon $A$ remains massless, while the other electroweak gauge bosons $Z$ and $W^{ \pm}$acquire the masses:

$$
\begin{aligned}
& m_{W}=\frac{g v}{\sqrt{2}}, \\
& m_{Z}=\frac{g v}{\sqrt{2} \cos \theta_{W}} .
\end{aligned}
$$

The Higgs gauge eigenstates can be expressed in terms of the Higgs mass eigenstates. In particular, the neutral Higgs gauge eigenstates can be decomposed in terms of the VEVs $\left(v_{u}, v_{d}\right)$, the CP-even mass eigenstates $\left(h^{0}, H^{0}\right)^{T}$ and the CP-odd mass eigenstates $\left(G^{0}, A^{0}\right)^{T}$ :

$$
\left(\begin{array}{c}
H_{u}^{0} \\
H_{d}^{0}
\end{array}\right)=\left(\begin{array}{l}
v_{u} \\
v_{d}
\end{array}\right)+\frac{1}{\sqrt{2}} R_{\alpha}\left(\begin{array}{c}
h^{0} \\
H^{0}
\end{array}\right)+\frac{i}{\sqrt{2}} R_{\beta_{0}}\left(\begin{array}{c}
G^{0} \\
A^{0}
\end{array}\right),
$$

whereas the charged Higgs gauge eigenstates can be expressed in terms of the charged +1 scalar $H^{+}$and the charged Golstone boson $G^{+}:^{1}$

$$
\left(\begin{array}{c}
H_{u}^{+} \\
H_{d}^{-*}
\end{array}\right)=R_{\beta_{ \pm}}\left(\begin{array}{c}
G^{+} \\
H^{+}
\end{array}\right)
$$

Here $R_{\alpha}, R_{\beta_{0}}$ and $R_{ \pm}$are the orthogonal rotation matrices:

$$
\begin{gathered}
R_{\alpha}=\left(\begin{array}{cc}
\cos \alpha & \sin \alpha \\
-\sin \alpha & \cos \alpha
\end{array}\right), \\
R_{\beta_{0}}=\left(\begin{array}{cc}
\sin \beta_{0} & \cos \beta_{0} \\
-\cos \beta_{0} & \sin \beta_{0}
\end{array}\right), \quad R_{\beta_{ \pm}}=\left(\begin{array}{cc}
\sin \beta_{ \pm} & \cos \beta_{ \pm} \\
-\cos \beta_{ \pm} & \sin \beta_{ \pm}
\end{array}\right),
\end{gathered}
$$

which are chosen to diagonalize the squared-masses of the quadratic part of the potential. In the tree-level approximation one finds that $\beta_{0}=\beta_{ \pm}=\beta, m_{G^{0}}^{2}=m_{G^{ \pm}}^{2}=0$ and the Higgs masses read:

$$
\begin{aligned}
m_{h^{0}, H^{0}}^{2} & =\frac{1}{2}\left(m_{A^{0}}^{2}+m_{Z}^{2} \mp \sqrt{\left(m_{A^{0}}^{2}-m_{Z}^{2}\right)^{2}+4 m_{Z}^{2} m_{A^{0}}^{2} \sin ^{2} 2 \beta}\right) \\
m_{A^{0}}^{2} & =2 \mu^{2}+m_{H_{u}}^{2}+m_{H_{d}}^{2} \\
m_{H^{ \pm}}^{2} & =m_{A^{0}}^{2}+m_{W}^{2}
\end{aligned}
$$

where the mixing angle $\alpha$ is determined by the equalities:

$$
\frac{\sin 2 \alpha}{\sin 2 \beta}=-\left(\frac{m_{H^{0}}^{2}+m_{h^{0}}^{2}}{m_{H^{0}}^{2}-m_{h^{0}}^{2}}\right), \quad \frac{\tan 2 \alpha}{\tan 2 \beta}=\left(\frac{m_{A^{0}}^{2}+m_{Z}^{2}}{m_{A^{0}}^{2}-m_{Z}^{2}}\right),
$$

\footnotetext{
${ }^{1}$ Here we define $G^{-}=G^{+}$and $H^{-}=H^{+}$. Also, by convention, $h^{0}$ is lighter than $H^{0}$.
} 
and is traditionally chosen to be negative; it follows that $-\pi / 2<\alpha<0$ (provided $m_{A_{0}}>m_{Z}$ ).

In the MSSM decoupling limit, i.e. for $m_{A}^{0} \gg m_{Z}$ and so for a large value of the parameter $\mu$, the particles $A^{0}, H^{0}$ and $H^{ \pm}$are very heavy and decouple from the low-energy effective model. Only the lightest Higgs particle $h^{0}$ stays at the electroweak scale. In this case, by using the relations of Eq.(1.28), the mixing angle becomes $\alpha \simeq \beta-\pi / 2$ and the lightest Higgs boson $h^{0}$ obtains the couplings of the ordinary SM Higgs boson.

\section{Neutralinos and Charginos}

In the R-parity conserving MSSM, winos $\tilde{W}^{a}$ and binos $\tilde{B}$ are mixing with the down-type higgsino $\tilde{H}_{d}$ and up-type higgsino $\tilde{H}_{u}$ due to the electroweak symmetry breaking in order to form the four electrically neutral mass eigenstates called neutralinos $\tilde{\chi}_{\alpha}^{0}$ and the two electrically charge mass eigenstates called charginos $\tilde{\chi}_{\alpha}^{ \pm}$.

Concerning the electrically neutral gauginos and higgsinos, the Lagrangian in the gauge eigenstate basis $\psi^{0}=\left(\tilde{B}, \tilde{W}^{0}, \tilde{H}_{u}^{0}, \tilde{H}_{d}^{0}\right)^{T}$ is given by:

$$
\mathcal{L}_{\text {neutralino mass }}=-\frac{1}{2} \psi^{0 T} M_{N} \psi^{0}+\text { h.c. },
$$

where the symmetric neutralino mass mixing matrix takes the form:

$$
M_{N}=\left(\begin{array}{cccc}
M_{1} & 0 & -\frac{g^{\prime} v_{d}}{\sqrt{2}} & \frac{g^{\prime} v_{u}}{\sqrt{2}} \\
0 & M_{2} & \frac{g v_{d}}{\sqrt{2}} & -\frac{g v_{u}}{\sqrt{2}} \\
-\frac{g^{\prime} v_{d}}{\sqrt{2}} & \frac{g v_{d}}{\sqrt{2}} & 0 & -\mu \\
\frac{g^{\prime} v_{u}}{\sqrt{2}} & -\frac{g v_{u}}{\sqrt{2}} & -\mu & 0
\end{array}\right)
$$

Introducing an orthogonal matrix, we can rewrite the above matrix in the basis of the supersymmetric partners of the massive gauge bosons: $\psi^{0^{\prime}}=\left(-i \tilde{\gamma},-i \tilde{Z}^{0}, \tilde{H}_{u}^{0}, \tilde{H}_{d}^{0}\right)^{T}$. It yields:

$$
\left(\begin{array}{c}
-i \tilde{\gamma} \\
-i \tilde{Z} \\
\tilde{H}_{u}^{0} \\
\tilde{H}_{d}^{0}
\end{array}\right)=R\left(\begin{array}{c}
-i \tilde{B} \\
-i \tilde{W}^{3} \\
\tilde{H}_{u}^{0} \\
\tilde{H}_{d}^{0}
\end{array}\right) \quad \text { with } \quad R=\left(\begin{array}{cccc}
c_{W} & s_{W} & 0 & 0 \\
-s_{W} & c_{W} & 0 & 0 \\
0 & 0 & 1 & 0 \\
0 & 0 & 0 & 1
\end{array}\right)
$$

The neutralino mass term can then be reformulated as:

$$
\mathcal{L}_{\text {neutralino mass }}=-\frac{1}{2} \psi^{0^{\prime T}} M_{N}^{\prime} \psi^{0^{\prime}}+\text { h.c. },
$$

where the neutralino mass matrix in the new basis can be written as

$$
M_{N}^{\prime}=R M_{N} R^{T}=\left(\begin{array}{cccc}
M_{1} c_{W}^{2}+M_{2} s_{W}^{2} & \left(M_{2}-M_{1}\right) s_{W} c_{W} & 0 & 0 \\
\left(M_{2}-M_{1}\right) s_{W} c_{W} & M_{1} s_{W}^{2}+M_{2} c_{W}^{2} & -m_{Z} s_{\beta} & m_{Z} c_{\beta} \\
0 & -m_{Z} s_{\beta} & 0 & -\mu \\
0 & m_{Z} c_{\beta} & -\mu & 0
\end{array}\right)
$$

Note that we have used the Equations (1.2), (1.18), (1.19), (1.21), (1.22) and the abbreviations $s_{W}=\sin _{W}, c_{W}=\cos _{W}, s_{\beta}=\sin _{\beta}, c_{\beta}=\cos { }_{\beta}$ to obtain the previous expressions.

The basis can be changed to the neutralino mass eigenstate basis: $\psi^{0^{\prime \prime}}=\left(\tilde{\chi}_{1}^{0}, \tilde{\chi}_{2}^{0}, \tilde{\chi}_{3}^{0}, \tilde{\chi}_{4}^{0}\right)^{T}$, by following a procedure similar to the one described above. In order to obtain positive mass 
eigenvalues one also has to add an additional diagonal unitary phase matrix. In doing so, the transformation into the neutralino basis reads:

$$
\left(\begin{array}{c}
\tilde{\chi}_{1}^{0} \\
\tilde{\chi}_{2}^{0} \\
\tilde{\chi}_{3}^{0} \\
\tilde{\chi}_{4}^{0}
\end{array}\right)=T\left(\begin{array}{c}
\tilde{\gamma} \\
\tilde{Z}^{0} \\
\tilde{H}_{d}^{0} \\
\tilde{H}_{u}^{0}
\end{array}\right)
$$

where $T$ denotes the composition of the transformation matrix and the diagonal phase matrix. The neutralino mass matrix is now diagonalized:

$$
T^{*} M_{N}^{\prime} T^{\dagger}=\left(\begin{array}{cccc}
m_{\tilde{\chi}_{1}^{0}} & 0 & 0 & 0 \\
0 & m_{\tilde{\chi}_{2}^{0}} & 0 & 0 \\
0 & 0 & m_{\tilde{\chi}_{3}^{0}} & 0 \\
0 & 0 & 0 & m_{\tilde{\chi}_{4}^{0}}
\end{array}\right) .
$$

Concerning the electrically charged gauginos and higgsinos, the spectrum can be analyzed in a similar way. In the gauge eigenstate basis $\psi^{ \pm}=\left(\tilde{W}^{+}, \tilde{H}_{u}^{+}, \tilde{W}^{-}, \tilde{H}_{d}^{-}\right)^{T}$, the mass terms in the Lagrangian are:

$$
\mathcal{L}_{\text {chargino mass }}=-\left(\psi^{ \pm}\right)^{T} M_{\tilde{C}} \psi^{ \pm}+\text {h.c. }
$$

where, in $2 \times 2$ block form,

$$
M_{\tilde{C}}=\left(\begin{array}{cc}
0 & X^{T} \\
X & 0,
\end{array}\right)
$$

with

$$
X=\left(\begin{array}{cc}
M_{2} & g v_{u} \\
g v_{d} & \mu
\end{array}\right)=\left(\begin{array}{cc}
M_{2} & \sqrt{2} m_{W} s_{\beta} \\
\sqrt{2} m_{W} c_{\beta} & \mu
\end{array}\right) .
$$

Note that we have employed the previous Equations (1.2), (1.21) and (1.22) to obtain the last equality of Equation (1.38). We can now change the basis to the mass eigenstates through the $2 \times 2$ unitary matrices $U$ and $V$ :

$$
\left(\begin{array}{c}
\tilde{\chi}_{1}^{-} \\
\tilde{\chi}_{2}^{-}
\end{array}\right)=U\left(\begin{array}{c}
\tilde{W}^{-} \\
\tilde{H}_{d}^{-}
\end{array}\right), \quad\left(\begin{array}{c}
\tilde{\chi}_{1}^{+} \\
\tilde{\chi}_{2}^{+}
\end{array}\right)=V\left(\begin{array}{c}
\tilde{W}^{+} \\
\tilde{H}_{u}^{+}
\end{array}\right) .
$$

The matrix $X$ can be diagonalized by $U$ and $V$ as follows:

$$
U^{*} X V^{\dagger}=V X^{\dagger} U^{T}=\left(\begin{array}{cc}
m_{\tilde{\chi}_{1}^{ \pm}} & 0 \\
0 & m_{\tilde{\chi}_{2}^{ \pm}}
\end{array}\right) .
$$

The chargino masses are given by the positive roots of the eigenvalues of $X^{\dagger} X$ :

$$
V X^{\dagger} X V^{\dagger}=U^{*} X X^{\dagger} U^{T}=\left(\begin{array}{cc}
m_{\tilde{\chi}_{1}^{ \pm}}^{2} & 0 \\
0 & m_{\tilde{\chi}_{2}^{ \pm}}^{2}
\end{array}\right)
$$


with

$$
m_{\tilde{\chi}_{1,2}^{ \pm}}^{2}=\frac{1}{2}\left(M_{2}^{2}+\mu^{2}+2 m_{W}^{2} \mp \sqrt{\left(M_{2}^{2}+\mu^{2}+2 m_{W}^{2}\right)^{2}-4 \mu M_{2}-m_{W}^{2} \sin 2 \beta^{2}}\right) .
$$

We remark that this treatment of the neutralino and chargino mass matrices is only valid at tree level and typically gets corrections from higher-order contributions.

\section{Stops}

As already said, the soft terms introduce a lot of new parameters in the theory. In particular, if we introduce gaugino and scalar masses and soft trilinear terms $A_{i}$, the squared-mass matrix for the top squarks in the gauge-eigenstate basis $\left(\tilde{t}_{L}, \tilde{t}_{R}\right)$ can be obtained. It is given by:

$$
\mathcal{L}_{m_{\tilde{t}}}=-\left(\tilde{t}_{R}^{*}, \tilde{t}_{L}^{*}\right) M_{\tilde{t}}^{2}\left(\begin{array}{c}
\tilde{t}_{L} \\
\tilde{t}_{R}
\end{array}\right)
$$

where

$$
M_{\tilde{t}}^{2}=\left(\begin{array}{cc}
m_{\tilde{t}_{R}}^{2} & m_{\tilde{t}}\left(A_{t}+\mu \cot \beta\right) \\
m_{\tilde{t}}\left(A_{t}+\mu \cot \beta\right) & m_{\tilde{t}_{L}}^{2}
\end{array}\right)
$$

is a non-diagonal hermitian matrix where $A_{t}, m_{t}$ and $\tan \beta$ denote, respectively, the trilinear coupling of the Higgs with top sfermions, the top quark mass, and the ratio of the two Higgs vacuum expectation values $\tan \beta=v_{u} / v_{d}$. The masses $m_{\tilde{t}_{R}}^{2}$ and $m_{\tilde{t}_{L}}^{2}$ arise from the soft breaking, the D term contribution, and the top Yukawa coupling as follows:

$$
\begin{aligned}
& m_{\tilde{t}_{R}}=m_{\tilde{U}_{3}}^{2}+m_{t}^{2}+\frac{2}{3} \sin ^{2} \theta_{W} m_{Z}^{2} \cos 2 \theta \\
& m_{\tilde{t}_{L}}=m_{\tilde{Q}_{3}}^{2}+m_{t}^{2}+\left(\frac{1}{2}-\frac{2}{3} \sin ^{2} \theta_{W}\right) m_{Z}^{2} \cos 2 \theta
\end{aligned}
$$

where $\theta_{W}$ denotes the weak mixing angle and $m_{Z}$ is the $Z_{0}$ boson mass. The soft breaking masses $m_{\tilde{U}_{3}}$ and $m_{\tilde{Q}_{3}}$ are model-dependent. We see that in general the stop mass matrix can have a large off-diagonal entry, in particular if $A_{t}$ is chosen large to explain the Higgs mass [14-16]. In such a case the two mass eigenstate repel each other, so that the lightest one can become much lighter than the average mass scale. The stop mass matrix $M_{\tilde{t}}^{2}$ can be diagonalized by a unitary matrix to give mass eigenstates:

$$
\left(\begin{array}{l}
\tilde{t}_{1} \\
\tilde{t}_{2}
\end{array}\right)=\left(\begin{array}{cc}
\cos \theta & -\sin \theta \\
\sin \theta & \cos \theta
\end{array}\right)\left(\begin{array}{c}
\tilde{t}_{L} \\
\tilde{t}_{R}
\end{array}\right)
$$

where $\theta$ is the stop mixing angle and $\tilde{t}_{1}$ is regarded the lighter stop mass eigenstate.

Note that this discussion is particularly relevant for this thesis since in Chapter 3 we investigate Stop NLSP decays at LHC both within R-parity parity conserving and R-parity violating SUSY and with a gravitino LSP and DM candidate. More precisely, in this chapter we do not study a particular model, but just assume that the NLSP is the lightest stop $\tilde{t}_{1}$ and that $\tilde{t}_{2}$ and the rest of the supersymmetric particles are outside the reach of LHC. Nevertheless, most of our results are weakly dependent on the stop mixing angle and therefore also valid if the second stop is not too heavy, as long as its production is suppressed. In case other colored states like 
gluino and first two generations squarks are within the LHC reach, we have additional particle production channels and the search becomes more promising.

In view of completeness, we mention that different SUSY breaking scenarios can account for a stop NLSP with a gravitino LSP. For instance, in gauge-mediated supersymmetry breaking (GMSB) scenarios [17] the supersymmetry-breaking scale is typically much smaller than in the gravity-mediated case and, thus, the gravitino is almost always the LSP. Moreover, in the recently proposed model-independent framework of general gauge mediation (GGM) $[18,19]$ any MSSM superpartner can practically be the NLSP and in extended models the measured Higgs mass can be also obtained [20-22], especially if the scale of supersymmetry breaking and the superpartner masses are high.

\section{R-parity}

One additional ingredient of the MSSM is the conservation of R-parity. R-parity is a multiplicative quantum number, defined for each particle as:

$$
P_{R}=(-1)^{3(B-L)+2 s}
$$

where $s$ is the spin of the particle under consideration. $B$ and $L$ instead are the baryon number and the lepton number of such a particle, respectively.

Defining R-parity makes counting interactions very easy since all of the SM particles have even R-parity $\left(P_{R}=+1\right)$ and their SUSY partners odd R-parity $\left(P_{R}=-1\right)$. If R-parity is exactly conserved then there can be no mixing between sparticles and SM particles. Additionally each interaction vertex in the theory must contain and even number of $P_{R}=-1$ sparticles. This gives us some extremely useful phenomenological results:

- the LSP must be absolutely stable. As previously stated, if it is electrically and color neutral than LSP would be a very natural candidate for DM

- Each supersymmetric particle can only decay into an odd number of superparticles, plus SM particles

- Supersymmetric particles can only be generated pairwise from SM particles (e.g. at LHC)

Note that even though the LSP, being it a excellent DM candidate, makes SUSY a very interesting theory from the astrophysical point of view, this was not the originally motivation for R-parity. In fact, R-parity was first introduced in order to forbid lepton and baryon violating processes which could lead, for instance, to rapid proton decay. By doing so, such a symmetry could explain the observed $B-L$ conservation and so suppress the rate of proton decay.

The nature of the LSP in the MSSM is constrained by many observations. It cannot have a non-zero electric charge or color, or it would have condensed with baryonic matter to produce heavy isotopes, which are in conflict with the observations [23]. The stable LSP is so presumably electrically neutral and only weakly interacting. Consequently, the expected signature of SUSY particle production at colliders is missing energy, carried by undetected LSPs.

There are basically two possible DM candidates in MSSM: neutralino $(s=1 / 2)$ [24-26], sneutrino $(s=0)[27,28]$. Neutralino is the mixture of neutral gauginos and Higgsinos, and one of the most popular and well studied DM candidates; in fact it will be further discussed later. On the contrary, the (left-handed) sneutrino in the MSSM turns out not to be viable as DM. 
Such a left-handed particle has indeed a sizable coupling with the $\mathrm{Z}$ boson and this leads to a too large annihilation cross section and therefore to a too small relic abundance. Moreover, the scattering cross section off nuclei of this superparticle (also mediated by Z boson exchange) is so large that the direct detection experiments for DM would have already observed it [29].

There are two more DM candidates if we consider the well-motivated alternative LSP candidates beyond the MSSM: axino $(s=1 / 2)[30,31]$ and gravitino $(s=3 / 2)[32]$. Either of them could indeed provide the right amount of Cold Dark Matter (CDM) in the Universe if heavier than about $1 \mathrm{MeV}$ and then not a priori excluded. However, axino and gravitino belong to a class of super-weakly interacting massive particles, or superWIMPs (produced in the late decays of WIMPs), that, due to their extremely tiny interactions, could be very difficult or impossible to observe in direct and indirect DM searches. In particular, gravitino appearing (as the superpartner of the graviton) once SUSY is promoted from a global to a local symmetry (see Section 1.3) has couplings suppressed by the Planck scale. In addition, gravitino mass depends strongly on the SUSY-breaking scheme and can range from the eV scale to scales beyond the $\mathrm{TeV}$ region. Axino, on the other hand, shows up (as the superpartner of the axion) when we invoke the Peccei-Quinn (PQ) solution to the strong CP problem in supersymmetric models. Unlike gravitino, axino has couplings suppressed by the axion decay coupling $f_{a}$, i.e. the scale of Peccei-Quinn symmetry breaking. According to the model and the SUSY breaking scheme, the axino mass can range between the $\mathrm{eV}$ and the $\mathrm{GeV}$ scale.

Although the superpotential $W$ of Equation (1.9) is sufficient by itself to produce a phenomenologically viable model, there are several other superpotential terms, consistent with all of the gauge symmetries, that can be added to it. So much so that, the most general MSSM superpotential can also contain any or all of the following terms that violate R-parity:

$$
W_{\not P_{R}}=\frac{1}{2} \lambda_{i j k} L^{i} L^{j} E^{c k}+\lambda_{i j k}^{\prime} L^{i} Q^{j} D^{c k}+\frac{1}{2} \lambda_{i j k}^{\prime \prime} U^{c i} D^{c j} D^{c k}+\mu_{i} H_{u} L^{i}
$$

As in the case of the R-parity conserving superpotential, a sum over the generation indices $i, j, k(=1,2,3)$ and the suppressed gauge indices is taken for granted. The R-parity breaking mass parameter $\mu_{i}$ and the trilinear Yukawa couplings $\lambda, \lambda^{\prime}$ violate the lepton number, whereas the coupling $\lambda^{\prime \prime}$ violates the baryon number. Hence, we can rewrite these terms by separating the baryon violating part:

$$
W_{\Delta B \neq 0}=\lambda_{i j k}^{\prime \prime} U^{i} D^{j} D^{k}
$$

from the lepton violating one:

$$
W_{\Delta L \neq 0}=\lambda_{i j k} L^{i} L^{j} E^{k}+\lambda_{i j k}^{\prime} L^{i} Q^{j} D^{k}+\mu_{i} L^{i} H_{u} .
$$

These two new terms lead to a significantly different phenomenology compared to that one of the R-parity conserving case. In more detail, the production of one single sparticle is now possible and the LSP is no longer stable (nodal point for the decays studied in this thesis). Nevertheless, there are very stringent constraints on these new couplings and parameters. For instance, constraints coming from the non-observation of proton decay and an existing baryon asymmetry in the early Universe. The lower bound on the proton lifetime $\left(\tau_{p}>10^{29}\right.$ years [33]), indeed, demands that either the $\mathrm{L}$ violating or the $\mathrm{B}$ violating couplings vanish, or that all the couplings are extremely small, if not zero. The existing baryon asymmetry in the early Universe 
before the electroweak phase transition, on the other hand, implies that the violating lepton number terms to be small (i.e. $\lambda, \lambda^{\prime}<10^{-7}[34]$ ).

There is no longer difference between Higgs and matter supermultiplets in R-parity violating models. For this reason, in such models the mixing of sleptons-Higgs, neutrinos-neutralinos and charged leptons-charginos are also allowed.

The first three terms of Equation (1.49) are the so-called trilinear R-parity violating part of the most general MSSM superpotential, which now reads as follows:

$$
W_{M S S M}=W+W_{P_{R}} .
$$

\section{Bilinear R-parity}

The forth term of Equation (1.49):

$$
W_{b R P V}=\mu_{i} \hat{H}_{u} \hat{L}_{i}
$$

instead, corresponds to the bilinear R-parity violation [35], and involves only three extra parameters, one $\mu_{i}$ for each generation. These three parameters have dimensions of mass and explicitly violate the lepton number. Their origin (and size) can be naturally explained in extended models where the breaking of lepton number is spontaneous.

The bilinear R-parity violating term $W_{b R P V}$ of Equation (1.52) is motivated by its ability to generate a hierarchical neutrino mass spectrum favored by observations (e.g. the solar neutrino problem). In fact, besides the Giudice-Masiero mechanism [36], such a R-parity violation is generated in those models where R-parity is spontaneously broken through the Vacuum Expectation Value (VEV) of the right-handed sneutrinos $v_{R} \neq 0$ and so, $\mu_{i}$ 's are identified as a product of a Yukawa coupling and $v_{R}$.

The presence of the new superpotential terms also implies new soft Supersymmetry breaking terms containing bilinear R-parity violation:

$$
\mathcal{L}_{\text {soft }}=\left(B_{i} H_{u} \tilde{L}_{i}+\tilde{m}_{d i}^{2} H_{d}^{\dagger} \tilde{L}_{i}+\text { h.c. }\right)+\ldots
$$

The 6 parameters $B_{i}$ and $m_{d i}^{2}$ are mass mixing terms with dimensions of mass squared.

In order to analyze the complete superpotential including the R-symmetry breaking terms, it is convenient to perform the following rotation of the Higgs $H_{d}$ and lepton $L_{i}$ supermultiplets:

$$
L_{i}^{\prime}=L_{i}-\epsilon_{i} H_{d}, \quad H_{d}^{\prime}=H_{d}+\epsilon_{i} L_{i}, \quad \text { with } \quad \epsilon_{i}=\frac{\mu_{i}}{\mu},
$$

where the parameters $\epsilon_{i}$ are the bilinear R-parity breaking parameters. By doing so, the bRPV term, Equation (1.52), can be rotated away from the superpotential. Nevertheless, in this new basis, R-parity breaking is reintroduced in form of trilinear R-parity violation in the Yukawa sector. In fact, one obtains the new trilinear R-parity violating terms:

$$
\Delta W^{\prime}=h_{i j k} L_{i}^{\prime} L_{j}^{\prime} E_{k}^{c}+h_{i j k}^{\prime} L_{i}^{\prime} Q_{j} D_{k}^{c}
$$

where

$$
h_{i j k}=-y_{e} \eta_{i j} \epsilon_{k}+y_{e} \eta_{k j} \epsilon_{i}, \quad h_{i j k}^{\prime}=-y_{d} \eta_{i j} \epsilon_{k} .
$$


We notice that even though this rotation generates new trilinear couplings, it does not lead to baryon number violation and so, proton remains stable.

In the soft scalar Lagrangian only the R-parity violating mass mixing terms, Equation (1.53), change at first order in the small R-parity breaking parameters $\zeta_{i}$ :

$$
B_{i}^{\prime}=B_{i}-\zeta_{i} B, \quad m_{d i}^{\prime 2}=m_{d i}^{2}+\epsilon_{i}\left(m_{i j}^{2}-m_{d}^{2}\right) \quad \text { with } \quad m_{i j(d)}^{2}=m_{\tilde{L}_{i j}\left(H_{d}\right)}^{2} .
$$

As it is well known, the VEVs for the sneutrino fields can then be found by looking at the minimum of the scalar potential in the sneutrino directions. In particular, in this new basis it turns out to be:

$$
\frac{v_{i}}{v_{d}} \simeq \frac{B_{i} \tan \beta-m_{d i}^{* 2}}{m_{i j}^{2}+\frac{1}{2} m_{Z}^{2} \cos 2 \beta}
$$

where we have dropped the primes on the rotated parameters and $\tan \beta$ refers to Equation (1.15). Note that this expression for the vacuum expectation values of the sneutrino fields has been used in several investigations on gravitino dark matter to parametrize the effect of bilinear R-parity violation.

One can perform an additional rotation of the fields in the scalar sector such that the sneutrino VEV vanishes if a different parametrization of bilinear R-parity breaking is taken into account. In doing so, although all effects of bilinear R-parity violation are encoded in the form of R-parity breaking Yukawa couplings, physics does not change its predictions.

Before introducing Supergravity, we point out that the bilinear R-parity violation will play a central role in the study of the R-parity violating stop decay with displaced vertices, discussed in Chapter 3.

\subsection{Supergravity}

At the time that Supersymmetry is promoted to a local symmetry, i.e. the parameter in SUSY transformations becomes coordinate-dependent, the theory necessarily implies gravity. Indeed, likewise gravity can be seen as the "gauge theory" of the global space-time transformation, the local Supersymmetry can be seen as the gauge theory of global supersymmetry. By this correspondence, local supersymmetry is also called Supergravity (SUGRA).

SUGRA unifies the space-time symmetries of ordinary general relativity with local supersymmetry transformations and, at the same time, conserves the invariance under local SUSY transformations by adding a new supermultiplet to the theory: The gravity supermultiplet, which consists of the spin- 2 graviton and the spin- $3 / 2$ gravitino. Such a new supermultiplet is shown in Table 1.4. As we can see from this table, gravitino, as well as graviton, is neutral with respect to the SM gauge groups. Moreover, in the case of unbroken Supergravity, it is also massless, i.e. it has only two transverse helicity states.

Although SUGRA is a very nice theory addressing the problem to let gravity enter into the Standard Model of particle physics, there is not a full quantum theory of gravity yet. Indeed,

\begin{tabular}{lccc}
\hline Name & Bosons & Fermions & $\left(S U(3)_{c}, S U(2)_{L}\right)_{Y}$ \\
\hline Graviton, gravitino & $g_{\mu \nu}$ & $\psi_{\mu}$ & $(\mathbf{1}, \mathbf{1})_{0}$ \\
\hline
\end{tabular}

TABLE 1.4: Gravity supermultiplet. 
Supergravity is nonrenormalizable in the same way as gravity. Its coupling constant $\kappa$ is still the dimensionful Newton's constant $\kappa \sim G_{N}^{1 / 2}$ and interactions scale like powers of $\kappa$. For this reason, SUGRA can be seen as an effective theory of a more fundamental one of gravity having the Planck scale $M_{P}=\left(G_{N} / \hbar c\right)^{-1 / 2} \sim 10^{19} \mathrm{GeV}$ as its reference scale. Note that the reduced Planck mass $M_{P_{r}}=\left(8 \pi G_{N} / \hbar c\right)^{-1 / 2} \sim 10^{18} \mathrm{GeV}$ is often used in particle physics and cosmology since the factor $(8 \pi)^{-1 / 2}$ simplifies a number of equations in GR.

In this thesis, however, we are interested in couplings of the gravitino to MSSM particles that are suppressed by the Planck mass, therefore we are not going to work in the so-called "flat limit" $\left(M_{P} \rightarrow \infty\right)$, where renormalizability is restored.

\section{Supergravity Breaking and Super-Higgs Mechanism}

Analogous to the Higgs mechanism of electroweak symmetry breaking we have discussed earlier, a Super-Higgs mechanism of Supersymmetry breaking comes into playing in Supergravity. It occurs when the massless Goldstone fermion of Supersymmetry breaking, the goldstino, is swallowed by the massless gravitino. In this way, spin- $3 / 2$ gravitino obtains its longitudinal (helicity $\pm 1 / 2$ ) components and becomes massive.

This is due to the gravitino-goldstino mixing mass term that is contained in the spontaneously broken supergravity Lagrangian. Invariance of the gravitino and the goldstino fields under local supersymmetry transformations demands a redefinition of the both fields. The redefined gravitino is then a linear combination of the gravitino and the goldstino, and therefore gets all four helicity states. In this case the gravitino mass becomes:

$$
m_{3 / 2} \simeq \frac{\langle F\rangle}{M_{P}}
$$

with $\langle F\rangle$ the non-vanishing $\mathrm{F}$-term vacuum expectation value of the hidden sector auxiliary field, responsible for the spontaneous breaking of the Supersymmetry (Equation (1.12)). This simple form for $m_{3 / 2}$ follows simply from dimensional analysis, since this mass value must vanish in the limits that supersymmetry is restored $(\langle F\rangle \rightarrow 0)$ and that gravity is turned off $\left(M_{P} \rightarrow \infty\right)$.

Equation (1.59) implies very different expectations for the gravitino mass depending on the particular scheme of SUSY breaking mediation. Basically, two are the best-known mechanisms for the mediation of Supersymmetry breaking: The gravity-mediated Supersymmetry breaking and the gauge-mediated Supersymmetry Breaking.

In gravity mediation, Supersymmetry is broken spontaneously in a hidden sector and mediated to the observable sector via non-renormalizable Planck mass suppressed interactions. Here, for dimensional reasons, the required value of the gravitino mass should range from the electroweak scale to $\mathrm{TeV}$ scale. In gauge mediation, instead, the supersymmetry breaking is mediated to the visible sector via a sector of messenger particles that couple to the MSSM particles via gauge interactions. In this instance, dimensional reasons lead to a gravitino mass whose value is in general much smaller than in the case of gravity mediation. Thus, the gravitino is always the lightest particle.

Regardless of the specific SUSY breaking mechanism, we take the gravitino mass as a variable parameter that is expected to be about $\mathcal{O}(10-100) \mathrm{GeV}$ in order to account for the dark matter density of the Universe. 


\section{Gravitino}

In classical field theory, the Lagrangian describing a massive gravitino $\psi_{\mu}$ propagating freely in space-time takes the following form:

$$
\mathcal{L}_{\text {free }}=-\frac{1}{2} \varepsilon^{\mu \nu \rho \sigma} \bar{\psi}_{\mu} \gamma^{5} \gamma_{\nu} \partial_{\rho} \psi_{\sigma}-\frac{1}{4} m_{3 / 2} \bar{\psi}_{\mu}\left[\gamma^{\mu}, \gamma^{\nu}\right] \psi_{\nu},
$$

where $\gamma_{\mu}=\left\{\gamma^{0}, \gamma^{1}, \gamma^{2}, \gamma^{3}\right\}$ are the Dirac matrices, $\gamma^{5}=i \gamma^{0} \gamma^{1} \gamma^{2} \gamma^{3}$ is the fifth Dirac matrix and, finally, $\varepsilon^{\mu \nu \rho \sigma}$ is the totally anti-symmetric tensor $\epsilon_{0123}=-1$ in flat space-time.

The field equation for the free gravitino can be obtained by varying the above Lagrangian for $\psi_{\mu}$, namely by applying the so-called principle of least action. By doing so, we achieve:

$$
\begin{aligned}
0 & =\left\{\left(\frac{\partial}{\partial \psi_{\mu}}\right)-\partial_{\nu}\left(\frac{\partial}{\partial\left(\partial_{\nu} \psi_{\mu}\right)}\right)\right\} \mathcal{L}_{\text {free }} \\
& =\frac{1}{2} \epsilon^{\mu \nu \rho \sigma} C^{\dagger} \gamma_{5} \gamma_{\nu} \partial_{\rho} \psi_{\sigma}+\frac{1}{4} m_{3 / 2} C^{\dagger}\left[\gamma^{\mu}, \gamma^{\nu}\right] \psi_{\nu} \\
& =\frac{1}{2} \epsilon^{\mu \nu \rho \sigma} \gamma_{5} \gamma_{\nu} \partial_{\rho} \psi_{\sigma}+\frac{1}{4} m_{3 / 2}\left[\gamma^{\mu}, \gamma^{\nu}\right] \psi_{\nu},
\end{aligned}
$$

in which the Majorana condition for the gravitino field (i.e. $\psi_{\mu}=C \bar{\psi}_{\mu}^{T}$ ) has been used. The last line, which denotes the equation of motion for a non-interacting gravitino, can lead to a simpler set of equations (Rarita-Schwinger equations) after some mathematical manipulation. In order to achieve this, we first apply to the last line of Eq.(1.61) either $\partial_{\mu}$ or the identity:

$$
\gamma_{\mu} \varepsilon^{\mu \nu \rho \sigma}=-i \gamma^{5}\left(\gamma^{\nu} \gamma^{\rho} \gamma^{\sigma}-g^{\rho \sigma} \gamma^{\nu}+g^{\nu \sigma} \gamma^{\rho}-g^{\nu \rho} \gamma^{\sigma}\right)
$$

the two equations:

$$
\begin{aligned}
m_{3 / 2}\left(\not \partial \gamma^{\nu} \psi_{\nu}-\gamma^{\nu} \not \partial \psi_{\nu}\right) & =0 \\
i\left(\gamma^{\mu} \not \partial \gamma^{\nu} \psi_{\nu}-\gamma^{\mu} \partial^{\nu} \psi_{\nu}+\not \partial \psi^{\mu}-\gamma^{\nu} \partial^{\mu} \psi_{\nu}\right)+m_{3 / 2}\left(\gamma^{\mu} \gamma^{\nu} \psi_{\nu}-\psi^{\mu}\right) & =0
\end{aligned}
$$

are easily found after few mathematical steps. Then we multiply Eq.(1.64) by the matrix $\gamma_{\mu}$ so as to obtain:

$$
i\left(\not \partial \gamma^{\nu} \psi_{\nu}-\gamma^{\nu} \not \partial \psi_{\nu}\right)+3 m_{3 / 2} \gamma^{\nu} \psi_{\nu}=0
$$

Eventually, by using the Eq.(1.63)-(1.65), Rarita-Schwinger equations for the massive gravitino field can be derived:

$$
\begin{aligned}
\gamma^{\mu} \psi_{\mu}(x) & =0, \\
\left(i \not \partial-m_{3 / 2}\right) \psi_{\mu}(x) & =0 .
\end{aligned}
$$

These equations yield the further constraint

$$
\partial^{\mu} \psi_{\mu}(x)=0
$$


Their adjoint equations can be also straightforward extracted:

$$
\begin{aligned}
\bar{\psi}_{\mu}(x) \gamma^{\mu} & =0, \\
i \partial_{\nu} \bar{\psi}_{\mu}(x) \gamma^{\nu}+m_{3 / 2} \bar{\psi}_{\mu}(x) & =0, \\
\partial^{\mu} \bar{\psi}_{\mu}(x) & =0 .
\end{aligned}
$$

In order to solve Rarita-Schwinger equations and then get their solutions, we move onto the momentum space, as is customary usage in classical and quantum field theory. In this space, positive and negative frequency solutions are found:

$$
\psi_{\mu}(x)=\psi_{\mu}^{+s}(p) e^{-i p \cdot x}, \quad \psi_{\mu}(x)=\psi_{\mu}^{-s}(p) e^{i p \cdot x} \quad \text { with } \quad s= \pm \frac{3}{2}, \pm \frac{1}{2} .
$$

The mode functions $\psi_{\mu}^{+}(p), \psi_{\mu}^{-}(p)$ must obey the corresponding Rarita-Schwinger equations in the momentum space $\left(p_{\mu}\right.$-space), which read:

$$
\begin{aligned}
\gamma^{\mu} \psi_{\mu}^{+s}(p) & =0, & \gamma^{\mu} \psi_{\mu}^{-s}(p) & =0, \\
\left(\not p-m_{3 / 2}\right) \psi_{\mu}^{+s}(p) & =0, & \left(\not p+m_{3 / 2}\right) \psi_{\mu}^{-s}(p) & =0 \\
p^{\mu} \psi_{\mu}^{+s}(p) & =0 & p^{\mu} \psi_{\mu}^{-s}(p) & =0 .
\end{aligned}
$$

Following the standard procedure, these modes can be constructed by using the tensor product of the familiar Dirac spinors of spin- $1 / 2$ particles and the polarization vector of a massive spin-1 particle. Moreover, by imposing the normalization of the Dirac spinors and the polarization vectors as well, we can derive the normalization of these gravitino modes.

$$
\begin{aligned}
& \bar{\psi}_{\mu}^{+s}(p) \psi^{+s^{\prime} \mu}(p)=-2 m_{3 / 2} \delta^{s s^{\prime}} \\
& \bar{\psi}_{\mu}^{-s}(p) \psi^{-s^{\prime} \mu}(p)=2 m_{3 / 2} \delta^{s s^{\prime}} .
\end{aligned}
$$

For the sake of completeness, we should also mention the gravitino polarization tensor:

$$
\begin{aligned}
P_{\mu \nu}^{ \pm}(p)=\sum_{s} \psi_{\mu}^{ \pm s}(p) \bar{\psi}_{\nu}^{ \pm s}(p) & =-\left(\not p \pm m_{3 / 2}\right)\left\{\Pi_{\mu \nu}(p)-\frac{1}{3} \Pi_{\mu \sigma}(p) \Pi_{\nu \lambda}(p) \gamma^{\sigma} \gamma^{\lambda}\right\} \\
\text { with } \quad \Pi_{\mu \nu}(p) & =\left(g_{\mu \nu}-\frac{p_{\mu} p_{\nu}}{m_{3 / 2}^{2}}\right) \quad \text { and } s=\left\{ \pm \frac{3}{2}, \pm \frac{1}{2}\right\}
\end{aligned}
$$

and its properties:

$$
\begin{aligned}
\gamma^{\mu} P_{\mu \nu}^{ \pm}(p) & =0, & P_{\mu \nu}^{ \pm}(p) \gamma^{\nu} & =0, \\
p^{\mu} P_{\mu \nu}^{ \pm}(p) & =0, & P_{\mu \nu}^{ \pm}(p) p^{\nu} & =0, \\
\left(\not p \mp m_{3 / 2}\right) P_{\mu \nu}^{ \pm}(p) & =0 & P_{\mu \nu}^{ \pm}(p)\left(\not p \mp m_{3 / 2}\right) & =0 .
\end{aligned}
$$

Notice that such a polarization tensor is very useful to compute unpolarized decay rates. For more details on this topic, see [37]. 
Let us now discuss the interactions between the gravitino and the MSSM fields. The interaction part of the gravitino Lagrangian is:

$$
\begin{aligned}
\mathcal{L}_{i n t}= & -\frac{i}{\sqrt{2}}\left[\left(D_{\mu}^{*} \phi^{i *}\right) \bar{\psi}_{\nu} \gamma^{\mu} \gamma^{\nu} P_{L} \chi^{i}-\left(D_{\mu} \phi^{i}\right) \bar{\chi}^{i} P_{R} \gamma^{\nu} \gamma^{\mu} \psi_{\nu}\right] \\
& -\frac{i}{8} \bar{\psi}_{\mu}\left[\gamma^{\nu}, \gamma^{\rho}\right] \gamma^{\mu} \lambda^{(\alpha) a} F_{\nu \rho}^{(\alpha) a}+\mathcal{O}\left({ }^{-2}\right) .
\end{aligned}
$$

where $\phi^{i}$ and $\chi^{i}$ are the complex scalar field and the left-handed two component Weyl fermion, respectively, of the MSSM chiral supermultiplets. By looking at this Lagrangian, we can see the covariant derivative of scalar fields:

$$
D_{\mu} \phi_{i}=\partial_{\mu} \phi_{i}+i \sum_{\alpha=1}^{3} g_{\alpha} A_{\mu}^{(\alpha) a} T_{a, i j}^{(\alpha)} \phi_{j},
$$

the chirality projection operators:

$$
P_{L}=\frac{1-\gamma^{5}}{2}, \quad P_{R}=\frac{1+\gamma^{5}}{2}
$$

and the field strength tensor for the gauge bosons:

$$
F_{\mu \nu}^{(\alpha) a}=\partial_{\mu} A_{\nu}^{(\alpha) a}-\partial_{\nu} A_{\mu}^{(\alpha) a}-g_{\alpha} f^{(\alpha) a b c} A_{\mu}^{(\alpha) b} A_{\nu}^{(\alpha) c} .
$$

In Equation (1.78), $T_{a, i j}^{(\alpha)}, \alpha=1,2,3$ are the generators of the Standard Model gauge groups:

$$
\begin{aligned}
T_{a, i j}^{(1)} & =Y_{i} \delta_{i j}, \\
T_{a, i j}^{(2)} & =\frac{1}{2} \sigma_{a, i j}, \\
T_{a, i j}^{(3)} & =\frac{1}{2} \lambda_{a, i j},
\end{aligned}
$$

where $Y_{i}$ is the hypercharge, $\sigma_{a}$ are the Pauli matrices, $\lambda_{a}$ are the eight Gell-Mann matrices and $f^{(\alpha) a b c}$ are the totally antisymmetric structure constants of the corresponding gauge group.

For the sake of completeness, we comment that the Feynman rules for gravitino, which can be extracted from the interaction Lagrangian (1.77) in the usual way, can be found in [38] and [39]. However, note that in Chapter 3 we will directly exploit the lagrangian (1.77) to investigate the R-parity conserving stop decay into top and gravitino and compute its decay rate.

\subsection{Minimal Dark Matter Model}

In this section we study a very simple model, capable of providing a good DM candidate. In particular, we consider is the minimal model introduced in [8] (see also [40-42] for simular setups) featuring a Majorana fermion $\psi$, singlet with respect to the SM gauge group and Dark Matter candidate, and a single scalar field multiplet $\Sigma_{f}$, non-trivially charged under at least one of the SM gauge groups. We assume that these fields interact among themselves and with the SM only via renormalizable Yukawa-type couplings according to the quantum number chosen for $\Sigma_{f}$. Note that possible LHC signals of such a model are investigated in Chapter 4 and 5 without introducing any symmetry to make DM stable. 


\section{Interactions}

The interaction between DM and the scalar $\Sigma_{f}$ is described by the effective lagrangian:

$$
L_{\mathrm{eff}}=\lambda_{\psi f} \bar{\psi} f \Sigma_{f}^{\dagger}+h . c .
$$

where $\psi$ is for the DM Majorana fermion, $f$ is for any chiral SM fermion, $\Sigma_{f}$ denotes a scalar with quantum numbers equal to $f$ and finally $\lambda_{\psi f}$ stands for the coupling between $\Sigma_{f}$ and $\psi$.

According to a minimality principle, only one $\Sigma_{f}$ field is introduced here. As a consequence DM only couple with quarks or leptons depending on whether $\Sigma_{f}$ carries color or only electroweak charge. Although we discuss the relevant mechanisms of DM production for this model only in the next chapter, we point out that the existence of the charged scalar $\Sigma_{f}$ is necessary for its production.

In absence of symmetries protecting the DM stability, interaction of the same type as above are also allowed between $\Sigma_{f}$ and two SM fermions. Depending on the quantum numbers a rather broad variety of operators may arise:

$$
\begin{array}{lrl}
L_{\mathrm{eff}}=\lambda_{1 q} \overline{\bar{l}} \ell \Sigma_{q}+h . c . & \Sigma_{q}=(3,2,1 / 3) \\
L_{\mathrm{eff}}=\lambda_{1 u} \bar{d} d^{c} \Sigma_{u}^{\dagger}+h . c . & \Sigma_{u}=(3,1,4 / 3) \\
L_{\mathrm{eff}}=\lambda_{1 d} \bar{q} \ell^{c} \Sigma_{d}+\lambda_{2 d} \bar{u} d^{c} \Sigma_{d}^{\dagger}+\lambda_{3 d} \bar{u} e^{c} \Sigma_{d}+\lambda_{4 d} \bar{q} q^{c} \Sigma_{d}^{\dagger}+h . c . & \Sigma_{d}=(3,1,-2 / 3) \\
L_{\mathrm{eff}}=\lambda_{1 \ell} \bar{\ell} \ell \Sigma_{\ell}+\lambda_{2 \ell} \bar{d} q \Sigma_{\ell}+\lambda_{3 \ell} \bar{u} q \tilde{\Sigma}_{\ell}+h . c . & \Sigma_{\ell}=(1,2,-1) \\
L_{\mathrm{eff}}=\lambda_{1 e} \bar{\ell} \ell^{c} \Sigma_{e}+h . c . & \Sigma_{e}=(1,1,-2)
\end{array}
$$

where $q, \ell$ denote the SM $S U(2)_{L} \mathrm{LH}$ doublets, while $u, d, e$ are $\mathrm{RH} S U(2)_{L}$ singlets, and the superscript ${ }^{c}$ indicates the charge-conjugated field, $f^{c}=C \bar{f}^{t}$ while $\tilde{\Sigma}_{f} \equiv i \sigma_{2} \Sigma_{f}^{*}$. On the right the quantum numbers of the $\Sigma_{f}$ fields are specified according to the SM gauge groups $S U(3)_{c} \times S U(2)_{L} \times U(1)_{Y}$. We are here suppressing flavour indices, even if some couplings like $\bar{q} q^{c}, \bar{\ell} \ell^{c}$ must be antisymmetric in flavour and vanish for a single generation, and considering in each line the presence of a single scalar field $\Sigma_{f}$. We have then that the new particle sector can just be described by two mass scales $m_{\psi}$ and $m_{\Sigma}$ and a few Yukawa couplings. The scalar field $\Sigma_{f}$ is also coupled, according to its assignment of quantum numbers, given above, to the SM group gauge bosons. In addition interactions with the Higgs boson of the form $|H|^{2}\left|\Sigma_{f}\right|^{2}$ or even $\left|H \Sigma_{q, \ell}\right|^{2}$ can be in general present. We assume, for simplicity, that such couplings are negligible, since they are not relevant for the processes which we are going to discuss in the next sections. We neglect as well any interaction like $\mu^{2} H \Sigma_{\ell}$, sticking only to dimensionless couplings, as well as the possibility that the neutral component of $\Sigma_{\ell}$, could acquire a v.e.v., which is instead similar to the case of bilinear RPV supersymmetry.

\section{Dark Matter indirect detection signatures}

The effective Lagrangians introduced above induce DM three-body decays into three SM fermions. Unless explicitly stated final state particles will be assumed massless. In this case the DM decay rate, up to kinematical and multiplicity factors, simplifies to:

$$
\Gamma_{\mathrm{DM}}=\frac{c_{f}|\lambda|^{2}\left|\lambda^{\prime}\right|^{2}}{384(2 \pi)^{3}} \frac{m_{\psi}^{5}}{m_{\Sigma_{f}}^{4}}, \begin{aligned}
& \lambda^{\prime}=\lambda_{i f} \\
& \lambda=\lambda_{\psi f}
\end{aligned}, \quad i=1, \cdots, 4, \quad f=q, u, d, \ell, e
$$


where $c_{f}$ counts the number of degrees of freedom of the intermediate $\Sigma_{f}\left(c_{f}=6,3,3,2,1\right.$ for $\Sigma_{q}, \Sigma_{u}, \Sigma_{d}, \Sigma_{\ell}, \Sigma_{e}$ respectively) while $\lambda^{\prime}$ stands for the coupling between $\Sigma_{f}$ and SM fermions only. The decay channels can be different depending on the quantum numbers of the intermediate particle $\Sigma_{f}$. We distinguish substantially four types of decay channels:

$$
\begin{array}{lll}
\psi \rightarrow \bar{u} u \nu, \bar{d} d \nu & \text { for } & \Sigma_{d}, \Sigma_{\ell} \\
\psi \rightarrow u \bar{d} \ell & \text { for } & \Sigma_{q}, \Sigma_{d} \\
\psi \rightarrow u d d & \text { for } & \Sigma_{u}, \Sigma_{d} \\
\psi \rightarrow \ell \bar{\ell} \nu & \text { for } & \Sigma_{\ell}, \Sigma_{e}
\end{array}
$$

For simplicity we focus on signals of the type $f \bar{f} \nu$ with $f$ being either a quark or a charged lepton of any generation, denoted by ell. We will also assume that the decays of DM are flavour conserving. If this is not the case bounds from flavour violating decays of mesons and leptons arise (see e.g. [43] for a complete list). However, they are sensitively weaker than the one imposed by cosmology and DM Indirect Detection (ID). Moreover, as above-mentioned, according to a minimality principle, for a given assignment of the quantum numbers of $\Sigma_{f}$, only one of the allowed operators, as reported in (1.83), dominates. We just comment that some of the effective lagrangian (1.83) violate both lepton and baryon number and in case of contemporary presence of lepton and baryon number violating operators, very strong constraints from the stability of the proton arise [44]. We will neglect here this possibility.

All the considered DM decay channels are already severely constrained from Indirect Detection. In fact, the hadronic decay channels, namely $d \bar{d} \nu$ and $u \bar{u} \nu$ are mostly constrained by antiproton searches, which give bounds on the DM lifetime varying between $10^{26}-10^{28} \mathrm{~s}$ for $m_{\psi}>100 \mathrm{GeV}$ [42] and can become as stringent as $10^{29}$ s for values of the DM mass down to $1 \mathrm{GeV}$ [45]. In the case of the leptonic decays, comparably severe constraints, ranging from approximately $10^{27} \mathrm{~s}$ to $10^{29} \mathrm{~s}$, according to the decay channel, for DM masses between $10 \mathrm{GeV}$ and $2 \mathrm{TeV}$, are obtained by the recent measurements by AMS of the positron flux and positron fraction [46]. In the case of the $\Sigma_{\ell}$ mediator, one-loop induced decay processes in $Z \gamma$ and $\nu \gamma$ may also be important. In particular the latter can originate monochromatic $\gamma$-ray lines. Current searches give bounds which can be as strong as $10^{29 \div 30}$ seconds for $m_{\psi}=1-10 \mathrm{GeV}[40,47,48]$. All these bounds can be satisfied only for a very small value of the product of the couplings, namely $\lambda \lambda^{\prime} \lesssim 10^{-(16 \div 22)}$, for masses of the scalar field $\Sigma_{f}$ within the kinematical reach of the LHC.

The potential correlation between DM Indirect Detection and collider signals is due to the fact that the same couplings $\lambda$ and $\lambda^{\prime}$, involved in the DM decay rate, induce decays of $\Sigma_{f}$ which can be observed at the LHC as well as, in the case of DM couplings with the quarks, the direct production of Dark Matter.

In order to study the impact of the ID limits on a possible detection of the scalar particle $\Sigma_{f}$ on a its possible detection, we assume that $m_{\Sigma_{f}}$ is the only relevant mass scale. Thus, its decay rate for a particular channel, up to kinematical factors, reads:

$$
\tilde{\Gamma}=\frac{\tilde{\lambda}^{2}}{8 \pi} m_{\Sigma_{f}}
$$




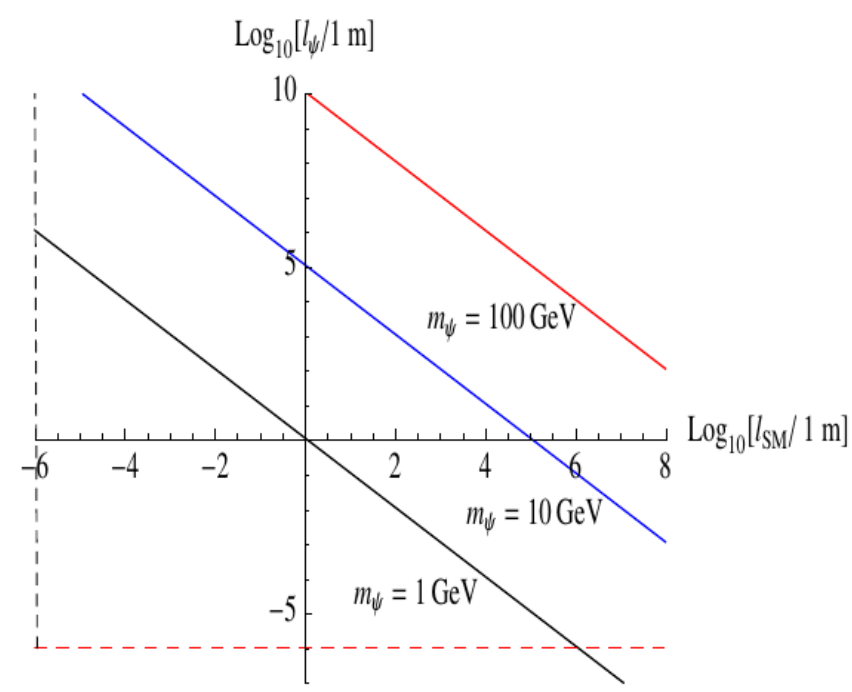

FIGURE 1.4: Value of the $\Sigma_{f}$ decay length into DM and one fermion vs. the decay length into SM states only, as imposed by Equation (1.89), ssuming the DM lifetime to be $10^{27} s . m_{\Sigma_{f}}$ is fixed at $1 \mathrm{TeV}$ while for $m_{\psi}$ we have chosen three sample values reported on the plot [8].

where $\tilde{\lambda}=\lambda, \lambda^{\prime}$ and $\tilde{\Gamma}=\Gamma, \Gamma^{\prime}$, respectively. Since we just have two decay channels of $\Sigma_{f}$, its total decay rate is simply given by the sum of the decay rates of the two channels:

$$
\Gamma_{\Sigma}=\Gamma+\Gamma^{\prime}=\frac{|\lambda|^{2}+\left|\lambda^{\prime}\right|^{2}}{8 \pi} m_{\Sigma}
$$

For the purpose of providing a better understanding of the coming pages, it is worth introducing here the branching ratio of the field $\Sigma$ into DM as a function of the two couplings:

$$
\operatorname{Br}\left(\Sigma_{f} \rightarrow f D M\right)=\frac{\Gamma}{\Gamma_{\Sigma}}=\frac{|\lambda|^{2}}{|\lambda|^{2}+\left|\lambda^{\prime}\right|^{2}} .
$$

By using the previous formulas we can express the rate (or equivalent the lifetime) of a given process of the type $\psi \rightarrow f \bar{f}^{\prime} f^{\prime \prime}$ in terms of the rates of the decays $\Sigma_{f} \rightarrow f \psi$ and $\Sigma_{f} \rightarrow \bar{f}^{\prime} f^{\prime \prime}$ or, more relevant for collider purposes, in terms of the decay lengths, up to eventual boost factors, $l=c \tau$.

We can relate the DM lifetime directly to the decay lengths of $\Sigma_{f}$ in the two channels:

$$
l_{\Sigma, D M}=\frac{c \hbar}{\Gamma\left(\Sigma_{f} \rightarrow f \psi\right)} \simeq 1.17 \mathrm{~m}\left(\frac{m_{\Sigma_{f}}}{1 \mathrm{TeV}}\right)^{-6}\left(\frac{m_{\psi}}{1 \mathrm{GeV}}\right)^{5}\left(\frac{l_{\Sigma, S M}}{1 \mathrm{~m}}\right)^{-1}\left(\frac{\tau_{\psi}}{10^{27} \mathrm{~s}}\right) .
$$

We see therefore that for a DM lifetime at the edge of detection, $\Sigma_{f}$ decay lenghts within the LHC detectors and of a similar order of magnitude can be achieved. Depending on the masses of the DM and of the scalar field $\Sigma_{f}$, it may therefore be possible to realize a scenario with a contemporary detection of both $\Sigma_{f}$ decays and with DM lifetime observable in the next future. In Figure 1.4 we show the relation given by Equation (1.89) for some sample values of the DM mass and assuming a reference value $m_{\Sigma_{f}}=1 \mathrm{TeV}$ and a DM lifetime of $10^{27} \mathrm{~s}$. 


\subsection{Experiment setup}

In this thesis we consider the LHC experiment with CMS as reference detector to investigate possible signatures (i.e. displaced and prompt decays) of the above-mentioned DM models in different DM cosmological scenarios. To this end, below we first describe very briefly the LHC experiment and then the CMS detector. Particularly, we notice how the CMS detector is made and what it can search for. For the purposes of clarity, from now we stress that even though we only deal with CMS, we expect the ATLAS detector to have comparable reach, perhaps even larger because of its bigger size.

\section{LHC experiment}

The Large Hadron Collider (LHC) is the world's largest and most powerful particle accelerator which started running on 10 September 2008. It was built by the European Organization for Nuclear Research (CERN) between 1998 and 2008 in collaboration with over 10,000 scientists from over 100 countries. It is till today the latest addition to CERN's accelerator complex.

Inside the accelerator, two high-energy particle beams travel at close to the speed of light before they are made to collide. The beams travel in opposite directions in separate beam pipes. They are guided around the accelerator ring (27-km ring) by a strong magnetic field maintained by superconducting electromagnets

On 13 February 2013 the LHC's first run (center of mass energy: $\sqrt{s}=8 \mathrm{TeV}$ ) ended, and it was shut down for planned upgrades. After "test" collisions, LHC officially restarted the second run on 3 June 2015 at $\sqrt{s}=13 \mathrm{TeV}$. After the discovery of the Higgs boson (July 4, 2012), LHC's aim is to continue to push our understanding of the fundamental structure of the universe. The results from LHC might indeed shed light on: Dark Energy, Dark Matter, Extra Dimension and Supersymmetry.

Seven experiments have been built at LHC and they all use detectors to analyse the large number of particles produced by collisions. The biggest of these experiments, ATLAS and CMS, use general-purpose detectors to investigate the largest range of physics possible. They have two independent detectors (ATLAS detector is bigger than CMS one) so as to cross-check possible discoveries. ALICE and LHCb have detectors specialized for focusing on specific phenomena, such as quark-gluon plasma and b-quark physics. These four detectors sit underground in huge caverns on the LHC ring.

The smallest experiments are TOTEM, LHCf and MoEDAL. Whereas TOTEM and LHCf focus on protons or heavy ions that brush past each other rather than meeting head on when the beams collide, MoEDAL search for a hypothetical particle called the magnetic monopole.

See a more complete discussion at http://home.web.cern.ch/about/experiments.

\section{CMS detector}

The CMS detector is built around a huge solenoid magnet. This takes the form of a cylindrical coil of superconducting cable that generates a field of 4 Tesla. The field is confined by a steel "yoke" that forms the bulk of the detector's 14,000-tonne weight.

It was build so as to reinforce SM and search for extra dimensions and Dark Matter candidates. Although it has the same scientific goals as the ATLAS experiment, it uses different technical solutions and a different magnet-system design. 


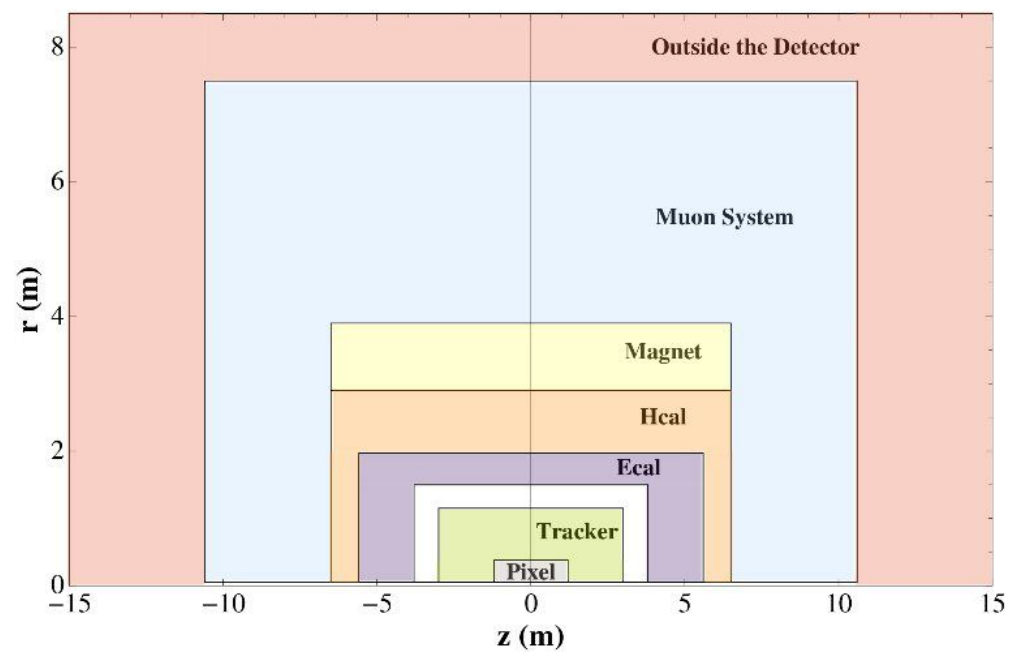

FIGURE 1.5: Layout of two quarters of CMS detector used in this analysis similar to [50]

The CMS detector uses a right-handed coordinate system where the origin is at the nominal interaction point. The $x$-axis points towards the centre of the LHC ring, the $y$-axis points up with respect to the plane of the LHC ring and, at last, the $z$-axis along the counterclockwise beam direction. The polar angle $\theta$ is measured from the positive z-axis, the azimuthal angle $\phi$ in the $x-y$ plane and the radial coordinate in this plane is denoted by $\mathrm{r}$. The transverse quantities, such as the transverse momentum $\left(\vec{p}_{T}\right)$, always refer to the components in the $x-y$ plane. In this context, the magnitude of the three-vector $\vec{p}_{T}$ is indicated by $\vec{p}_{T}$ and the transverse energy $E_{T}$ is defined as $E \sin \theta$.

In order to make easier the description of the CMS detector, the layout of one quarter of it was sketched in Figure 1.5. Now, if we start from the innermost part of the detector and going outwards, we can see the following parts: Interaction Point (IP), Pixel (Pi), Tracker (Tr), Electromagnetic Calorimeter (EC), Hadron Calorimeter (HC), Magnet (M), Muon System (MS). Below, a very short description of all of them is listed. A more detailed one can be found in [49].

- Interaction Point (IP) is the point in the centre of the detector at which proton-proton collisions occur between the two counter-rotating beams. We will assume that the stop and antistop pair is produced at this point.

- Pixel (Pi) detector contains 65 million pixels, allowing it to track the paths of particles emerging from the collision with extreme accuracy. It is also the closest detector to the beam pipe and, therefore, is vital in reconstructing the tracks of very short-lived particles. We therefore expect that the decay would be very well measured if it happens in this part of the detector.

- Tracker (Tr) can reconstruct the paths of high-energy muons, electrons and hadrons, as well as see tracks coming from the decay of very short-lived particles. It is also the second inner most layer and, so, receives (along with the Pixel) the highest number of particles. Even if it is less densely equipped than the Pixel detector, it can still recognize tracks coming from a displaced vertex instead than the interaction point. 
- Electromagnetic Calorimeter (EC) is designed to measure the energies of electrons and photons with high accuracy via electromagnetic calorimeters. In our case it can allow to measure the energy of the lepton arising in the decay.

- Hadron Calorimeter $(H C)$ measures the energy of hadrons and can give an estimate of the b-jet energy in the decay.

- Magnet $(M)$ is the central device around which the experiment is built. The job of this big magnet $(\vec{B}=4 T)$, which contains all the parts above, is to bend the paths of particles and allow for an accurate measurement of the momentum of even high-energy particles.

- Muon System $(M S)$ is able to detect muons and possibly other charged particles able to cross the whole detector.

The best detector parts to single out the presence of a displaced vertex are the pixel and tracker detectors and therefore we will restrict our discussion to the case of particle decaying there or surviving through the whole detector.

\section{Detector constraints}

In order to discuss the detector capabilities to identify the long-lived particles and the promptly decaying particles arising from our scenarios, we will consider the current available searches of detector stable and prompt particles, respectively, in Chapters 3, 4 and 5. There are basically two searches to take carefully into consideration. On one hand, we have the current CMS search for metastable particles, obtained at $\sqrt{s}=8 \mathrm{TeV}$ and $L=18.8 \mathrm{fb}^{-1}$, which excludes masses approximately below $800 \mathrm{GeV}$ since no significant excess of charged metastable particles has been observed from any of the its five performed complementary analyses/channels $[51,52]$. On the other hand, we have the current CMS searches for prompt scalar leptoquarks and the current ATLAS searches for prompt top quarks. In particular, the most severe constraints for scalar leptoquarks, obtained at $\sqrt{s}=8 \mathrm{TeV}$ and $L=19.6 \mathrm{fb}^{-1}$ from for the first generation scalar, exclude masses below approximately $840 \mathrm{GeV}$ [53], whereas the most severe ones for top quarks, obtained at $\sqrt{s}=8 \mathrm{TeV}$ and $L=20.1 \mathrm{fb}^{-1}$, exclude masses below approximately 750 $\mathrm{GeV}$ with the exception of the range $177 \lesssim m \lesssim 200 \mathrm{GeV}[54-56]$. 



\section{Chapter 2}

\section{Dark Matter}

"An era can be said to end when its basic illusion are exhausted"

Arthur Miller

Dark Matter (DM) is still one of the most exciting and tantalizing puzzle of modern cosmology and particle physics since it represents one of the strongest indications for physics beyond the current theory describing particle physics (SM). Its presence is well established at different scales, from galaxies to large scale structures and cosmological scales. However, despite the numerous and independent evidences, the nature of Dark Matter in not yet understood. In fact, the Dark Matter identification in terms of particle interpretation is still an open question for both cosmology and particle physics. Unlike normal matter, Dark Matter does not interact with the electromagnetic force at any significant level. This means it does not absorb, reflect or emit enough light to make Dark Matter detectable via electromagnetic radiation.

From astrophysical and cosmological observations, we have a very clear idea of the contribution of Dark Matter $(\sim 23 \%)$ to the energy budget of the Universe and what it is not. Although we will go back to the DM properties in the following pages, it is worth listing immediately such knowledge. Dark Matter is:

- Not baryonic

- Not made of Massive Compact Halo Objects (MACHOs)

- Not Hot Dark Matter (and therefore the only neutral massive SM particles, the neutrinos are unfortunately excluded...)

- Not collisional (possibly only interacting gravitationally, but maybe also participating in the weak interaction)

One of the most striking properties of DM particles related to their nature of being weakly interacting is their longevity, evidenced by their survival from the production in the early Universe to the present time. The only electrically neutral particles of the SM having such 
a long lifetime are the three neutrinos. Nevertheless, although their longevity can be easily explained by either the Lorentz symmetry (lightest neutrino) or the tiny masses (other two neutrinos), neutrinos cannot be Cold DM (the most significant component of the universe to explain the growth of structures such as galaxies and stars) exactly because of these tiny values of the masses. Hence, such longevity can only be justified within concrete DM models by additional symmetries imposed from the start.

Nowaday the particle interpretation of DM is thought to be one of the most persuasive ones. Moreover, it is believed that the DM particles would interact with baryonic matter and be light enough to be produced at LHC. Due to their weakly interactions, in case they were created at the LHC, they would escape through the detectors unnoticed. Nevertheless, they would carry away energy and momentum, so physicists could infer their existence from the amount of energy and momentum "missing" after a collision.

One among the most compelling above-mentioned theories is SUSY. Indeed, this theory, as already stressed in the previous chapter, besides being motivated by several reasons that go from theoretical reasons (e.g. hierarchy problem) to reasons linked to particle physics (e.g. naturalness), it is also motivated by the prediction of the existence of particles with properties expected from DM. However, SUSY is not the only explanation for DM. In recent years, indeed, other several exciting ideas raised in the theoretical physics community. For lack of time, hereunder we only list some of these alternative theories:

- "Extra" dimensions: Universe has more than three spatial dimensions. DM is a perfect candidate for matter that would exist in these "new" dimensions and that could only interact via gravity with the baryonic matter [57].

- "Hidden Valley": A parallel world made of DM having very little in common with the baryonic matter. According to this theory, DM is a "messenger" capable of crossing over the "Hidden Valley", escaping into the dark sector and becoming invisible to us $[58,59]$.

- "Modified gravity": Modification of the laws of gravity established by Newton and Einstein to account for DM [60].

Let us point out that if one theory of the previous ones proved to be true, it could help scientists to gain a better understanding of the composition of our Universe and, in particular, how galaxies hold together since they were formed.

In this Chapter, first we introduce Dark Matter from an historical perspective and then in the context of the Standard Cosmological Model $(\Lambda \mathrm{CDM})$. After that we are going to briefly present the current evidences for Dark Matter and thus its properties. Finally, we focus our attention on the DM particle interpretation, ignoring Extra Dimension and Hidden Valley theories, and study its different mechanisms of production.

Note that the author has used the references [39,61-68] during the draft of this subsection. Readers interested in this topic can find ample coverage in other works $[69,70]$.

\subsection{A short history of Dark Matter}

In order to understand deeply the scientific revolution we have obtained by introducing DM as a basic constituent of the Universe, we think that a brief historical introduction to DM is necessary. Let us point out that we summarize here the history of DM following the excellent 
works done by Sidney van den Bergh in [61,62]. Before going into detail of this exciting story, we would like to offer you a first taste of such a revolution by pointing out that in 1900 it was believed that almost $100 \%$ of the mass of the Universe resided in stars. Nowadays, instead, it is well known that these stars (and cold gas) account for only $\sim 1 \%$ its mass. Moreover, the remaining mass of the Universe is thought to reside in hot baryons $(\sim 3 \%)$, Cold Dark Matter $(\sim 22 \%)$ and Dark Energy $(\sim 74 \%)$, a new form of energy pervading the Universe.

In 1933 the term "Dunkle Materie" (lit: Dark Matter) was introduced for the first time by the Swiss astronomer Fritz Zwicky in his manuscript "Spectral displacement of extra galactic nebulae" [71]. It happened more or less a quarter of millennium after that Newton (1687) introduced in "PhilosophiceNaturalis Principia Mathematica" the concept of gravity in terms of forces between "bodies", i.e. visible baryonic objects.

Zwicky's first observations of the radial velocities of eight galaxies in the Coma cluster found an unexpectedly large velocity dispersion $\sigma=1019 \pm 360 \mathrm{Km} \mathrm{sec}^{-1}$. From these observations Zwicky concluded that the mean density of the Coma cluster would to have to be 400 times greater than that one which is derived from the luminous matter. Although Zwicky overestimated the mass-to-light ratio, he was right in claiming that some "Dunkle Materie" with a much greater density than luminous matter had to be present in the studied cluster and also in other cluster of galaxies.

Zwicky's manuscript has not had a very significant impact on astronomers during the first half of the 20th Century. Even as late as 1961 only a single paper at the "Santa Barbara Conference on the Instability of Systems of Galaxies" (Neyman, Page \& Scott 1961) referenced to the first Zwicky's manuscript on Dark Matter. This lack of references can not justified by neither the fact that Zwicky's research was written in German nor it was published in a relatively obscure (Helvetica Physica Acta) journal. In fact, in 1936 Sinclair Smith published a manuscript in English about an unexpected high mass [72], found in Virgo cluster and this manuscript was not cited by any of the other conference participants either.

It is noteworthy that the American astronomer Edwin Hubble (1936) was aware of the mass discrepancy problem in the Virgo cluster [73]. Nevertheless, it is not clear from his writings if he also knew about Zwicky's (1933) discovery of the missing mass problem in Coma.

The bibliographic database of the Institute for Scientific Information (ISI) for Zwicky's pioneering 1933 manuscript is shown in Table 2.1. Here the very low citation rate before 1975 (when the knowledge of the missing mass problem began to spread out) is evident. For the period 1934-1944 no official data are available yet. However, the two self-citations by Zwicky (1937, 1942) and the citation by Smith (1936) in his Virgo cluster manuscript were collected through a manual search of the Astrophysical Journal. A more up-to-date citations history is displayed in Figure 2.1.

\begin{tabular}{lc}
\hline Year & No. citations \\
\hline $1955-59$ & 2 \\
$1960-64$ & 6 \\
$1965-69$ & 5 \\
$1970-74$ & 2 \\
$1975-89$ & 63 \\
$1990-99$ & 71 \\
\hline
\end{tabular}

TABLE 2.1: Citations of Zwicky (1933) [61]. 
In the 1930s another set of astrophysical observations led to the "classic" evidence for dark matter: the rotational velocity curves of spiral galaxies. Astronomers found that the outer regions of galaxies were rotating faster than expected when a mass distribution based upon visible matter was assumed. The first observations of this discrepancy came in 1939 from the Andromeda galaxy, whereas only in the 1970s such observations were extended to larger radii. These years went down in history as "Dark Ages" due to the lack of enthusiasm and interest in these new astrophysical results. To this end, it is worth noting that the manuscripts on the "missing mass" in galaxy clusters and the manuscripts on the "galaxy rotation curves" of spiral galaxies were not connected to each other.

A turning point came in 1973 with Peebles and Ostriker's works. Indeed they showed that instabilities in models of galaxy disks could be solved by a massive spherical component, a socalled halo. Further, with Yahil they noted that the galaxy masses increase significantly with the radial distance from the galactic center, which in turn requires that rotational velocities remain high for large radial distances. These results, combined with the latest velocity curves at that time, provided a strong argumentation for the existence of "missing mass" in galaxies.

In the 1990s the era called "Dark Matter Renaissance" began. This name was chosen since in such a period a Renaissance of astrophysical results first confirmed and then refined scientifically the missing mass hypothesis, thus ruling out known possible reasonable alternatives. In addition, this decade brought several new astrophysical and cosmological methods/evidences to investigate the nature of this anomaly. Although we discuss them in detail later in this chapter, hereunder we list the most important ones.

- X-rays spectroscopy

- Gravitational lensing

- Cosmic Microwave Background (CMB)

- Large Scale Structure (LSS)

- Big Bang Nucleosynthesis (BBN)

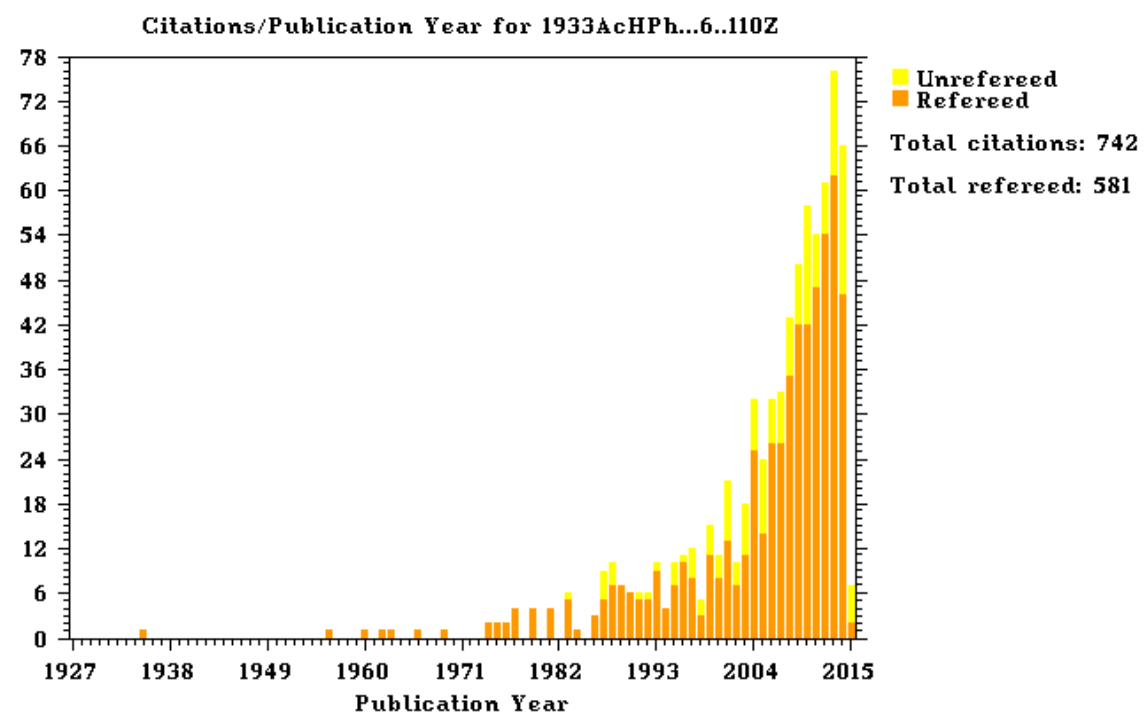

Figure 2.1: Histogram of Zwicky's citations history (1933) [74]. 
- Bullet Cluster (BC)

Note that all of these methods/evidences provide very stringent constraints on DM.

In closing, we remark from an historical perspective why we should not be surprised from the existence of DM in the Universe. Since the publication of Newton's most famous work "PhilosophiceNaturalis Principia Mathematica", the deviations of observed gravitational motions from expected Newtonian trajectories have proved to be very incisive to better understand our Universe. In fact, every time anomalies were seen in the motion of planets of the Solar system, theorists came up with two sorts of explanations. The first explanation consisted of considering incorrect Newton's law of gravitation, thus requiring some modifications of it. The second one, instead, consisted of leaving the theory unchanged and seeing such discrepancies as an indication of the existence of unseen objects. In the past, the second explanation was used by Urbain Le Verrier and John Couch Adams in the case of the anomalous motion of Uranus to conjecture the existence of the planet Neptune $[75,76]$. As time went by, this guesswork proved to be correct. On the contrary, the first explanation was used to attempt to justify the discrepancies in the motion of Mercury. Here, the existence of a new planet, called Vulcan, was also proposed but such a planet was never observed. This interpretation was, thus, wrong and we had to wait the advent of Einstein's theory of general relativity, i.e. the introduction of a more refined description of the gravitational laws, to obtain the final explanation.

\subsection{Evidences for the existence of Dark Matter}

Although DM has become such an established paradigm in modern physics that its existence is generally accepted with few explanations, it is worth reminding in the next few pages some of the strongest and most compelling evidences on which this paradigm actually stands. For this reason, we make a list of these evidences that is not meant to be an exhaustive one, but is just a sufficient sample of arguments to motivate DM searches.

\section{Kinematics}

Perhaps the most convincing and direct evidence for DM on galactic scales is a kinematic evidence: the observations of the rotation curves of galaxies, i.e. the graph of circular velocities of stars and gas as a function of their distance from the galactic center.

The observed rotation curves usually exhibit a characteristic flat behavior at large distances, even far beyond the edge of the visible disks, instead of decreasing as a function of the distance from the galactic centers as expected in Newtonian dynamics. In fact, the Newtonian dynamics asserts that the circular velocity of clusters of galaxies is given by:

$$
v(r)=\sqrt{\frac{G M(r)}{r},}
$$

where $M(r) \equiv 4 \pi \int \rho(r) r^{2} d r$ and function $\rho(r)$ denotes the mass density profile which should be falling $\propto 1 / \sqrt{r}$ beyond the optical disk.

The simplest explanation is that galaxies contain much more Dark than luminous matter. Indeed, if on one hand the luminous matter is that kind of matter that can be explained by the bright stellar objects residing in galactic disks, on the other hand, DM provides the force to speed up the orbits. Furthermore, by looking at Equation (2.1), the fact that $v(r)$ is 


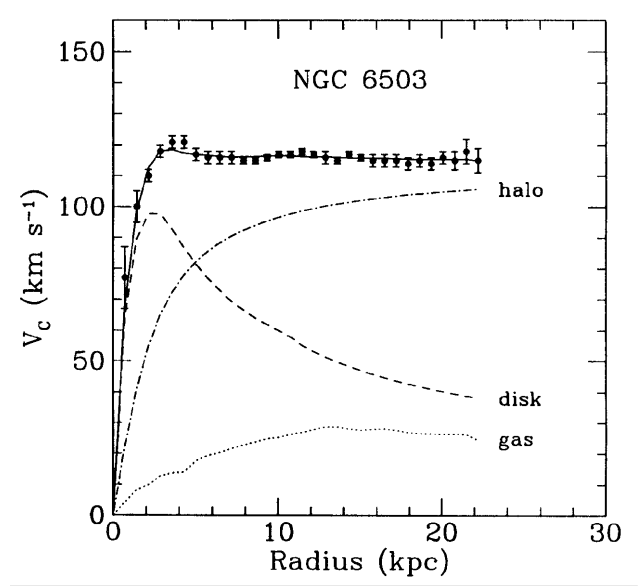

FiguRE 2.2: Rotational curve of NGC 6503 [7r]. The dotted, dashed and dot-dashed lines are the contributions of gas, disk and Dark Matter, respectively.

approximately constant can be justified by the presence of an additional enormous DM halo, much more extended than the observed stellar disk, with $M(r) \propto r$ and $\rho \propto 1 / r^{2}$. This is illustrated in Figure 2.2, where rotational curve of the dwarf spiral galaxy NGC 6503 is showed as a function of radial distance from the center of the galaxy. In particular, regarding this figure, the solid line displays the fit to data (dots with error bars) while the dashed, dotted and dash-dotted lines display the decomposition into the contributions from the luminous disk, gas and DM halo, respectively. It becomes clear that baryonic matter which accounts for gas and disk cannot alone explain the galactic rotation curve.

The limitations of rotation curves are that one can only look out as far as there is light or neutral hydrogen $(21 \mathrm{~cm})^{1}$, namely to distances of tens of kpc. Therefore, one can see the beginnings of DM haloes, but can not trace where most of DM is. The gravitational lensing evidences discussed in the next section go beyond these limitations.

At the present time, the rotational curves of all galaxies studied, including Milky way, are well-measured by several surveys which are all in agreement with Zwicky's finding that clusters contain more Dark than luminous matter.

\section{Gravitational lensing}

Gravitational lensing is another compelling tool to obtain the matter content of an object. According to GR, the presence of any mass causes the curvature of space-time, and so the curvature of geodesics, which results in bending the light rays around the massive bodies, such as galaxies or quasars. The light coming from distant and background objects, thus, experiences the bending from massive and foreground objects (or, in other words, it is "lensed" by them).

Gravitational lensing could also create multiple imagines of distance objects, or, if these imagines cannot be individually resolved, the background object could appear brighter. The main features of success of the lensing of DM until now is the evidence that DM is seen out to much larger distances than could be established by rotational curves. DM is seen in galaxies out of $200 \mathrm{kpc}$ from the centers of galaxies, in agreement with N-body simulations.

\footnotetext{
${ }^{1}$ The $21-\mathrm{cm}$ line produced by neutral hydrogen provides a useful probe to study the differential rotation of spiral galaxies. For more details, see: www.haystack.mit.edu/edu/undergrad/srt/SRT\%20Projects/rotation.html.
} 

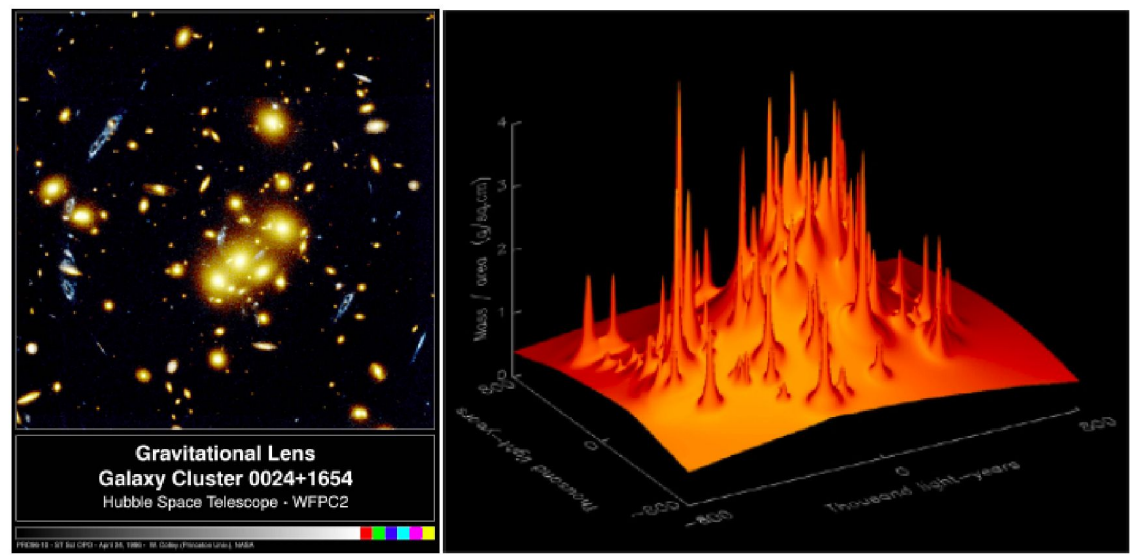

FiguRE 2.3: Left panel: Foreground cluster CL0024+1654 (yellow painted) and lensed image of the background cluster CL0024+1654 (blue painted). Right panel: A computer reconstructed mass map shows a large, broad peak around the center of the cluster which is not visible in the optical image, indicating the presence of a massive Dark Matter halo. These images have been taken by [78].

Another important achievement of gravitational lensing lies in the analysis of systems that are not in dynamical equilibrium and, thus, such an evidence for DM can be used as a relatively clean probe of the halo masses.

A beautiful example of a strong lens has been displayed in Figure 2.3. The panel on the left side shows several blue, loop-shaped objects that actually are multiple images of the same galaxy. They have been duplicated by the gravitational lens of the yellow, elliptical and spiral galaxy cluster - called $0024+1654$ - near the center of the photograph. The huge mass of the invisible Dark Matter in this cluster has, indeed, bent the light rays coming from the background galaxy. Moreover, the bits of white embedded in the blue galaxy and the dark core inside the ring represent young stars and dust (material used to make stars), respectively. The panel on the right side, instead, displays the mass distribution of the same galaxy as a two-dimensional orange surface, where the height ( $z$-axis) denotes the amount of mass at the corresponding point in the $x-y$ plane. A large and broad peak around the center of the cluster (clearly visible in the image) indicates the presence of a massive DM halo. In this computer reconstruction is therefore clear that the most of DM is not clinging to the galaxies in the cluster (the narrow and high peaks), but instead is smoothly distributed. Finally, note that the above-mentioned large and broad peak is, unsurprisingly, absent in the optical image on the left side.

\section{Bullet cluster}

Perhaps the most striking astrophysics evidence for non-baryonic DM is the so-called bullet cluster, shown in Figure 2.4. This object is in fact a double galaxy cluster which is believed to be the resulting collision of two independent subclusters. Here, while DM halos, deduced from gravitational lensing and painted blue, have passed straight through the collision point, the baryonic matter, deduced from X-ray gas map and painted pink, has slowed down because of the electromagnetic friction and, eventually, coalesced to a new region at the center of the bullet cluster. This shows that DM must not necessarily track the luminous matter and that it does not interact strongly with either gas or itself, in other words it is effectively collisionless. 


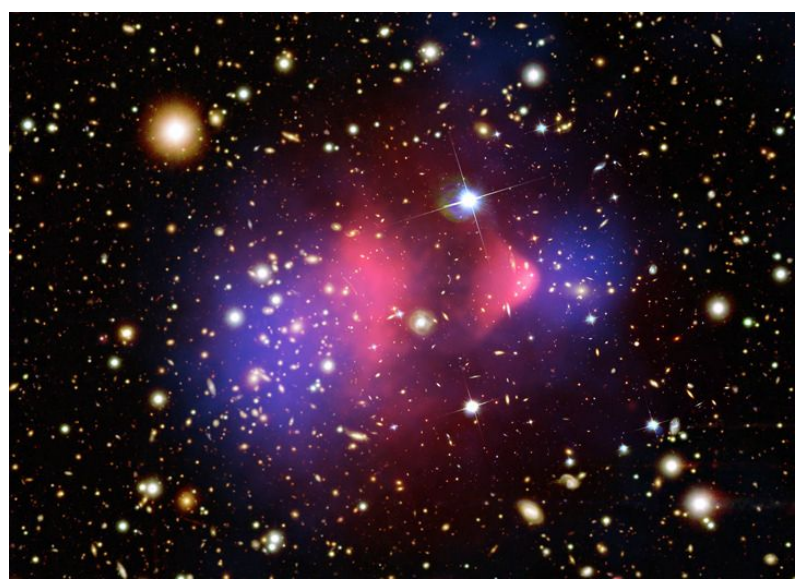

FIGURE 2.4: A collision of galactic clusters (bullet cluster) shows baryonic matter (pink) as separate from Dark Matter (blue), whose distribution is inferred by gravitational lensing [79].

To conclude, let us remark that such a surprising behavior observed in the Bullet cluster, in particular such a differentiation of the dark component from the baryonic one ${ }^{2}$, seems to be difficult to explain in terms of modified gravity theories without DM. Thus, this evidence reveals itself once again as one of the most compelling evidences for DM.

\section{Structure formation}

The formation of structures in our Universe provides us another important evidence of Dark Matter. On large scale, the Universe shows indeed an extremely complex structure: galaxies are gathered into clusters, clusters are part of superclusters and superclusters are arranged into large scale sheet, filaments and voids. This rich pattern has been formed by gravitational collapse which acts in opposition to the expansion of the Universe.

This collapse is different for baryonic matter and DM, since the former -made essentially of protons and electrons- interacts electromagnetically and thus has a non-negligible pressure against collapse. Instead DM feels no pressure and can amplify the fluctuations in the Cosmic Microwave Radiation and, consequently, the collapse.

Large scale cosmological N-body simulations prove that the observed large scale structure of luminous matter could only have been formed in the presence of a substantial amount of DM with several properties that we discuss later. Specifically, these simulations show that if the Universe had only made of baryonic matter, the structure formation would have started later due to the baryonic pressure.

\section{Cosmic Microwave Background}

The best estimation of the DM density, and so one of the best evidences, comes from the accurate study of the Cosmic Microwave Background (CMB) radiation. This isotropic radiation bath permeates the entire Universe and offers a glance at the Universe when it was only few hundred thousand old ( $T \simeq 0.25 \mathrm{eV}$ or $z \simeq 1100$ ), namely when the radiation (photons) decoupled from the thermal plasma of electrons and light elements. This radiation cooled as time passed by and the Universe expanded. Nowadays CMB has a temperature of 2.7 Kelvin.

\footnotetext{
${ }^{2}$ Notice that the computed ratio of hot gas mass to total mass $(1 / 6)$ is in very good agreement with best today estimations of $\Omega_{b_{o}} / \Omega_{m_{o}}$.
} 
The fact that the spectrum of the CMB radiation is almost the same as the one of a "black body" (a physicists way of describing a perfect radiator) implies that it could not have had its origin through any trivial process. This black-body behavior has, indeed, led to the death of the Steady State theory, according to which Universe has no start, and so matter is continuously created at a rate keeping the average density of the Universe constant as it expands.

Although the CMB radiation is practically isotropic, first the Cosmic Background Explorer (COBE) satellite mission and then Planck satellite mission observed and recorded light temperature anisotropies in the CMB sky map at the level of $\Delta T / T \sim 10^{-5}$.

Based on the measurements of Planck collaboration 2015, the density of baryonic matter $\Omega_{b_{o}}$ and the non-baryonic DM $\Omega_{C D M_{o}}$ are [80]:

$$
\Omega_{b_{o}} \simeq 0.04919, \quad \Omega_{C D M_{o}} \simeq 0.2636 .
$$

The overall matter content $\Omega_{m_{o}}=\Omega_{b_{o}}+\Omega_{C D M_{o}}$ of the Universe is so dominated by DM, which can be either baryonic or non-baryonic. Baryonic DM can contribute $10 \%$ or $15 \%$ of the total DM abundance in form of cold molecular gas or MAssive Compact Halo Objects (MACHOs); however, evidences reasonably strong, such as its small contribution to the mass of the galactic halo, show that it can not explain all amount of DM. We are going to be referring to the non-baryonic DM component throughout this thesis as simply DM.

Finally, we note that the angular scale along with the height of the peaks and troughs of the oscillations of the CMB radiation are powerful probes of several cosmological parameters besides the DM component, for instance, the total energy density and the baryonic fraction.

\subsection{Dark Matter ten-point test}

As already emphasized at the beginning of this Chapter, although DM had historically been inferred by many astronomical observations, its composition long remained speculative. Thus, identifying the nature of DM is one of the most important open problems in astroparticle physics today. In addition, although several DM interpretations in terms of modified gravity and hidden heavy normal objects (such as black holes, neutron stars, faint old white dwarfs, and brown dwarfs) have been proposed so far, the current evidences for DM seem to be in better agreement with its particle interpretation.

Standard Model of particle physics does not provide any explanation for DM because none of its particles is a good dark matter candidate. Most of its matter particles are, indeed, unstable, with lifetimes far shorter than the age of the Universe. On the other hand, the remaining particles are the six lightest ones: electron, up and down quarks, which can form stable protons and neutrons in nuclei, and finally the three neutrinos. Electrons can contribute significantly to DM only if they are neutralized through binding with protons, but protons (and neutrons) contribute to the baryonic energy density $\Omega_{B}$, which is too small to be all of DM. Massive neutrinos, supported by their oscillations, have instead a current upper bound on their masses which implies that the neutrino relic density is too small $\left(\Omega_{\nu} \simeq \sum_{i} m_{\nu_{i}} / 47 \mathrm{eV} \lesssim 0.012-14\right)$ to give an important contribution to the total DM density.

The fascinating connection between the DM problem and particle physics has suggested many new DM candidates, which are being searched for in accelerator, direct and indirect experiments at the present time. The improvement of our understanding of astroparticle physics along with the technical improvement of the running experiments reduce then the allowed regions in the 
DM particle parameter space little by little. In this section we glimpse the so-called "Ten-Point Test" that a new particle must overcome in order to be considered a good DM candidate. We notice that such a test was proposed by Marco Taoso, Gianfranco Bertone and Antonio Masiero in the manuscript [68]. Finally, we review the only DM candidates which has been taken into consideration in this thesis.

A particle can be thought of as a good DM candidate only if a positive answer can be given to all the following points:

1. Does it match the appropriate relic density?

2. Is it cold?

3. Is it neutral?

4. Is it consistent with BBN?

5. Does it leave stellar evolution unchanged?

6. Is it compatible with constraints on self-interactions?

7. Is it consistent with direct detection searches?

8. Is it compatible with gamma-ray constraints?

9. Is it compatible with other astrophysical bounds?

10. Can it be probed experimentally?

The distinction between the gamma-ray constraints and other astrophysical bounds just wants to stress the privileged role of photons in astrophysics. Photons, indeed, propagate along straight lines (being electrically neutral) and they can be detected with better precision than other particles (e.g. neutrinos). Additionally, the choice of gamma-ray photons comes from having energy in the range of energy where decay or annihilation of the majority of DM candidates occur. Note that the points 3. 4. 5. 6. and 7. of the above list are automatically satisfied if we take into account a DM candidate which interacts very weakly. In the end, we point out that although the last point of the above list is not a necessary condition, it is an essential point of the modern scientific method because a candidate that can not be detected, at least indirectly, would never be accepted as the solution to the DM evidences.

\section{Does it match the appropriate relic density?}

In order for a candidate to be considered a good DM particle, a production mechanism that reproduce the correct value of DM relic density must exist. Actually, there exist nowadays several viable mechanisms of DM production that lead to particle relics spanning a mass range of some thirty-three orders of magnitude. In this thesis we focus our attention specifically on the two mechanisms called SuperWIMP and Freeze-in. The former for the gravitino DM production whereas the latter for the Majorana fermion DM of the minimal model of decaying DM, introduced in Chapter 1. A short introduction of the phenomenology of both mechanisms is given in the following section along with a brief introduction of the WIMP mechanism.

Additionally, in order for a DM candidate to be considered a good DM particle, the lifetime of a promising candidate must be longer than the age of the Universe today (i.e. $\tau_{U} \gtrsim 10^{17}$ s) to survive from its production to the present-time. In several models such a stability of DM is ensure by imposing additional symmetries imposed from the start that forbid the DM candidate to decay. However, in absence of fundamental motivations to require the absolute 
stability of DM, models providing a DM candidate with a lifetime comparable with the age of the Universe are still well-motivated. We comment that the two models for DM particles presented in Chapter 1 belong to the latter class.

\section{Is it cold?}

The velocity of the DM particles at the time of equality between radiation and matter determines its ability not to be confined in halos and, therefore, its ability to erase the underlying density fluctuation. Moreover, it also sets at which scale the structures are able to collapse. In principle this characteristic can be used to constrain the non-baryonic DM to be either "cold", "warm" or "hot". Actually, the quantity related to it and used in astrophysics is the free-streaming length, that is the distance that DM particles can travel after decoupling.

Hot Dark Matter (HDM) refers to non-relativistic particles which are traveling with high velocities when the clumps that would form galaxies and clusters of galaxies began to grow. Event though they are not relativistic, HDM has a substantial free-streaming length at the time of matter-radiation equality because of its high velocities. This key feature causes three big problems with the validity of this type of DM particles. The first problem concerns their escaping capability from potential wells so that small density fluctuations will dissolve away before recombination, and so baryons will not collapse into small clumps. The second problem, instead, concerns the HDM models prediction of a top-down hierarchy in the formation of structures, with small structures being produced by fragmentation of larger ones. The third and last problem concerns the scale of these clumps. They are indeed as big as big clusters of galaxies, which have relatively low overdensities. In this way the collapse occurs too slowly and the following fragmentation even slower. Even though Hot Dark Matter models are today disfavored as the main contribution of DM, being them in completely disagreement with the observation, very small amounts of HDM are allowed. Classical examples for HDM candidates are massive neutrinos, whose masses in the $\mathrm{eV}$ regime their free-streaming length is of the order of the size of superclusters.

Cold Dark Matter (CDM), instead, refers to particles that were moving with negligible velocities when the pre-galactic clumps began to form. The prototype of CDM candidate is the supersymmetric neutralino, whose free-streaming length is such that only fluctuations roughly below the earth mass scale are suppressed. The best predictions for the behavior of CDM models come from N-body simulations, which are in general in good agreement with the observed large scale structures making such models the most widely accepted cosmological models at the present time. Nevertheless, some discrepancies with data have led some physicists to doubt CDM models and at the same time propose new explanations, among which we have the Warm Dark Matter paradigm. To this end, it is worth reminding the unobserved CDM predictions of small substructures embedded in larger objects, known as the "missing satellite" problem [81], and cuspy DM halo profile.

Warm Dark Matter (WDM), at last, refers to particles with speeds intermediate between $\mathrm{HDM}$ and CDM which is able to alleviate the above-mentioned discrepancies. The larger freestreaming length of WDM than the one of CDM reduces the power at small scales, inhibiting the formation of small structures. Prototypical examples are very light gravitino and sterile neutrinos. Observations of the Lyman- $\alpha$ forest give strong bounds on the mass of WDM sterile neutrinos, the free-streaming length of WDM particles and SuperWIMP scenarios (see Section 2.7). As regards the sterile neutrinos, we remark that such a WDM candidate arising from an extension of the minimal model of decaying DM of Chapter 1 will be discussed in Chapter 5 . 
In order to see directly such differences between CDM and WDM, it is very interesting to use the simulations of Libeskind and collaborators, displayed in Figure 2.5. These simulations, indeed, produce three objects named $\mathrm{A}, \mathrm{B}$ and $\mathrm{C}$ in decreasing mass at $z=0$ both in CDM (left picture) and WDM (right picture) scenario. Note that in the CDM run these objects have the same mass, geometry and kinematics as the Milky way galaxy (MW), Andromeda galaxy (M31) and Triangulum galaxy (M33). By comparing the CDM and WDM simulations of this figure, it is clearly visible their different structure formation, namely the delayed structure formation in WDM run in comparison with the structure formation in the CDM one. This is a direct consequence of the suppression of small scale power which, in terms of time evolution of structures, means a longer time for halos to grow to a given mass.

\section{Is it neutral?}

There are many reasons to believe that DM particles cannot possess an electric charge (or any other kind of charge). If they had a charge, their interaction rate with ordinary matter would be too large. Nevertheless, in principle it is possible that they might exist in form of bound states, for example in form of "heavy hydrogen", where a positively charged DM particle is surrounded by one electron. One of the most interesting constraints arise from searches for "heavy water" in lakes or oceans; that is a molecule characterized by HXO instead of $\mathrm{H}_{2} \mathrm{O}$, where one of the charged DM particles (CHAMPs) takes the place of one hydrogen. Since all the searches of anomalous hydrogen in the sea have failed, we are able to limit this scheme severely and effectively. Moreover, these charged DM particles are also constrained by balloon or satellite experiments for Cosmic Rays (CR) studies, underground experiments and, finally, stellar evolution. Note that even if these different constraints are not completely modelindependent, the combination of them basically rules out CHAMPs as DM. Even fractionary electric charged DM particles, known as milli-charged particles, cannot modify this outcome
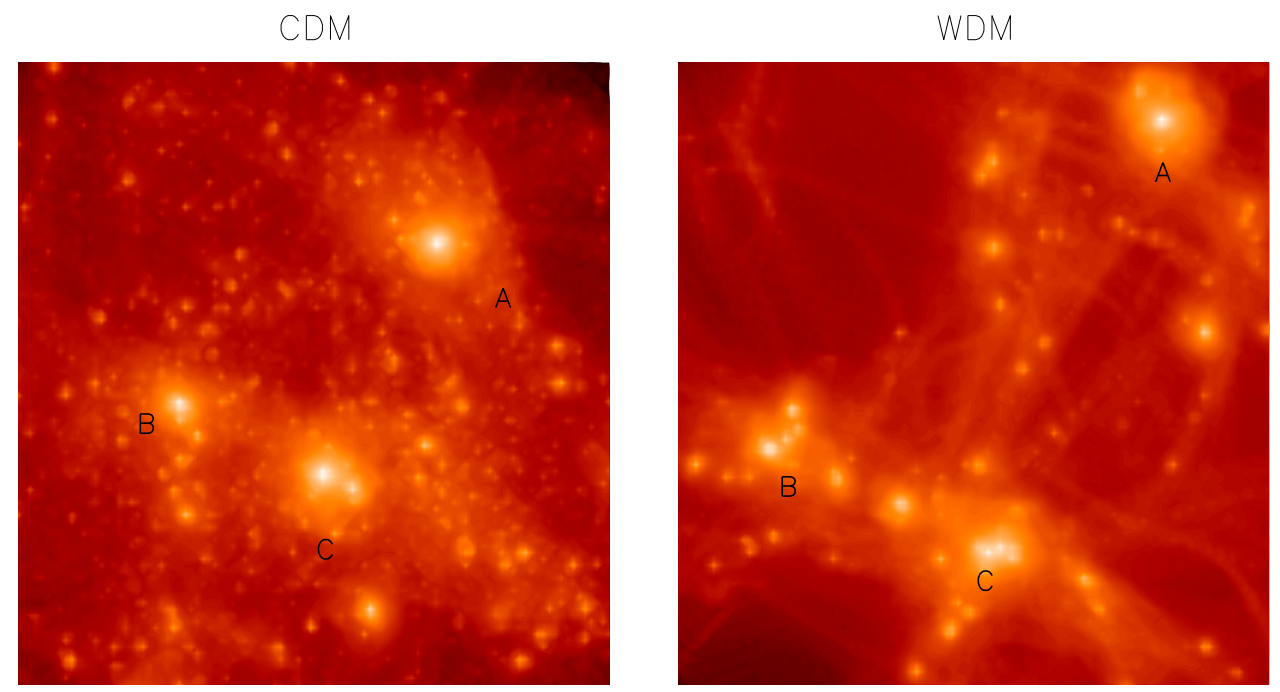

Figure 2.5: A density map containing three halos $A, B$ and $C$ that make up the simulated group at $z=0$ in CDM run (left) and WDM run (right). These two images have been taken by [82]. 
and thus become a good candidate. However, these constraints can be evaded by taking into account very massive composite DM candidates. For instance, macroscopically large nuggets of ordinary light quarks and/or antiquarks, with masses in the range $m \sim 10^{20}-10^{33} \mathrm{GeV}$, can behave as collisionless Cold Dark Matter in agreement with the observations [83].

\section{Is it consistent with BBN?}

In order not to spoil the very good agreement between the Big Bang Nucleosynthesis (BBN) theoretical predictions and the observations, the DM candidate must fulfill a series of severe constraints. In particular, among all constraints, it is worth reminding the one on the baryon-tophoton ratio $\eta$ to understand the importance of this theory. In the framework of the Standard Model, the observed abundances of light elements agree with predictions only if the baryon-tophoton ratio is in the range [84]:

$$
5.7 \times 10^{-10} \leq \eta \equiv \frac{n_{b}}{n_{\gamma}} \leq 6.7 \times 10^{-10} \quad(95 \% \mathrm{CL}) .
$$

Moreover, since such an abundance of baryons is also consistent with the estimate of CMB experiments given by WMAP and the most recent experiment Planck, BBN is considered one of the most impressive successes of Standard Cosmology. In conclusion, it is worth pointing out that the predictions of BBN can be dangerously modified by decays of particles during of after BBN. For this reason, studying decays of long-lived particles might create problems with the consistency of BBN, as we discuss more in detail in Chapter 3 where gravitino plays the role of DM and stop superparticle the long-lived NLSP.

\section{Does it leave stellar evolution unchanged?}

The fact that the nature of the DM particle could have an important effect on the stellar evolution was realized in the past 20 years. During this period, a rather precise understanding of stellar structure and its evolution has indeed been achieved and the agreement between the theory and observations could provide a powerful tool to constrain DM particles better and better. Nowadays the obtained bounds are even more important because they are often complementary to those coming from accelerator, direct and indirect searches, helping to rule out new parts of the parameter spaces. An example of these bounds, as discussed in more detail in [68], comes from the particles that could be collected in large quantities at the center of stars, including our Sun. In fact, these particles could lead to an accumulation of mass so large that they would collapse into a black hole, which would eventually destroy later the entire star. Clearly, since we do not see this event in any star, any combination of particle physics parameters that would destroy these stars can be ruled out.

\section{Is it compatible with constraints of self-interactions?}

As we have seen in the previous section, the Bullet Cluster provides a convincing evidence that most of the mass in the two colliding clusters is Dark. It is interesting that this system allows us to set a constraint on the self-interaction of DM. The subcluster DM halo (known as Bullet) would be indeed dragged by the main halo in presence of DM self-interactions, leading to an offset between the positions of visible galaxies (practically collisionless) and the total mass peak. 


\section{Is it consistent with direct Dark Matter searches?}

Direct detection of DM is a search strategy based on the interactions of Dark Matter particles with the nuclei of a detector, usually placed deep underground. The reason for placing these experiments in underground laboratories is to discriminate a DM signal against the natural background, coming from the copious cosmic radiation coming from space. These experiments have made huge technical progress in the last years. Therefore, the constraints arising from them are getting more and more complementary to those arising from accelerator experiments, thus ruling out more and more models which disagree with all available experiments.

\section{Is it compatible with gamma-ray constraints?}

Aside from direct and collider searches, DM particles can also be detected indirectly through the products of their annihilation or decay. Unlike direct detection, the indirect detection of DM particles aims at detecting the pale light which comes from the collision of two DM particles or simply from their decay. As regards the DM annihilations, since the energy scale of the annihilation photons is set by the DM mass and some of the most studied DM candidate are expected to be heavy ( $\mathrm{GeV}-\mathrm{TeV}$ is the mass range), exotic gamma-ray sources are among the primary targets of indirect detection. This process is more efficient where the density of DM is higher. Thus powerful telescopes are used to search for high energy photons, which are produced by large concentration of DM placing at the galactic center or in nearby galaxies. These constraints are particular relevant for this thesis. In particular, we will have to take into account the Indirect Detection constraints coming from gravitino decay when in Chapter 3 we investigate the R-parity violating stop decay at LHC. The gravitino decay, indeed, leads to a diffuse $\gamma$-ray flux which can be compared to that one measured by Fermi-LAT telescope in order to get bounds on the gravitino lifetime.

\section{Is it compatible with other astrophysical bounds?}

Besides gamma rays, it is possible to search for other particles arising from the annihilation or decay of DM particles, for instance, neutrinos, positrons, anti-protons and photons with smaller energy than gamma rays. Neutrinos can be generated by DM annihilations either directly or through the decay of other annihilation products. Positron can be originated from the decay of charged pions and kaons, which in turn are produced in cosmic ray interactions with interstellar gas. Anti-protons can be instead created from DM annihilations or decays in the galactic halo. Finally, observation of light with smaller energy than gamma rays (radio and X-rays) can be produced by DM annihilation and decay and it can give even more restrictive bounds than those inferred from gamma rays. There is currently a huge effort to understand the macroscopic properties of DM, and although a convincing detection has not been achieved, these techniques are useful at least for allowing us to rule out some theoretical possibilities. These constraints are also relevant for this thesis. In fact, in Chapter 4 we will deal with some hadronic and leptonic decay channels of scalar field $\Sigma_{f}$ of the minimal model of decaying DM of Chapter 1, which can be compared with the current anti-proton and positron searches so as to obtain constraints on the DM lifetime. 


\section{Can it be probed experimentally?}

As already mentioned, the nature of this requirement is different from the nine ones discussed above, in which we have practically demanded that the DM scenario is not in conflict with the existing experiments and observations. Indeed the nature of this requirement is related to the capability of present and future experiments to detect a good DM candidate at least indirectly. Thus, the experimental evidence is not really a necessary condition, in fact DM particles might be beyond the reach of any current and upcoming technology, but despite that they could exist. In order to obtain consistent result with the current collider searches, in this thesis we will exploit the LHC experiment, as our reference collider, and CMS, as our reference detector. Here we comment that, according to the scenarios under consideration, the current CMS bounds on metastable particle as well as the current CMS bounds on prompt decay will be imposed, respectively, in Chapters 3,4 and 5.

\subsection{Introduction to the Standard Cosmology Model}

In this section, first, we introduce the concept of metric along with Einstein's equations. Second, we use them to discuss briefly the dynamics of the Universe. Finally, we quickly describe the most important stages of the thermal history of our Universe.

\section{Einstein's equations in the Universe}

Gravitation can be described by a metric, a mathematical tool which turns coordinate distances into physical distances. In this way, instead of thinking of gravity as an external force and talking of particles moving in a gravitational field, we can include gravity in the metric and talk of particles moving freely in a distorted or curved space-time, namely a space-time where the metric can not be converted everywhere into Minkowskian form. In the $4 \mathrm{D}$ space-time, the invariant distance, i.e. the observer-independent distance, is given by:

$$
d s^{2}=\sum_{\mu, \nu=0}^{3} g_{\mu \nu} d x^{\mu} d x^{\nu},
$$

where the indices $\mu, \nu$ range from 0 to 3 , with 1 reserved for the time-like coordinate whereas $2,3,4$ for spatial coordinates. Because of the invariant nature of $d s^{2}$, the metric tensor $g_{\mu \nu}$ must be a covariant symmetric tensor: $g_{\mu \nu}=g_{\nu \mu}$.

The connection between the metric and the amount of matter and energy that fills the Universe (energy-matter content) is given by the equations of General Relativity (GR):

$$
G_{\mu \nu} \equiv R_{\mu \nu}-\frac{1}{2} g_{\mu \nu} R=8 \pi G_{N} T_{\mu \nu}
$$

better known as Einstein's equations. Here $G_{\mu \nu}$ is the Einstein tensor, $R_{\mu \nu}$ is the Ricci tensor which depends nontrivially on the metric and its derivatives, $R$ is the contraction of the Ricci tensor and it is called Ricci scalar, $G_{N}$ is Newton's constant and finally $T_{\mu \nu}$ is the energymomentum tensor, namely a symmetric tensor describing the constituents of the Universe.

Despite the simple appearance of the equations they are actually quite complicated. Given a specified distribution of matter and energy through the stress-energy tensor, Einstein's equations are a system of 10 coupled, nonlinear, hyperbolic-elliptic partial differential equations, that 
only depend on the metric $g_{\mu \nu}$. Therefore for gravitational fields the principle of superposition is not valid, contrary to the case for the electromagnetic field in the special theory of relativity. Nevertheless, one has usually to deal with weak gravitational fields, for which the Einstein's equations in first approximation are linear.

The energy-momentum conservation law can be expressed by the divergence of the energymomentum tensor as follows:

$$
T_{\mu \nu}^{; \nu}=0
$$

where " $; \nu$ " stands for the covariant derivative with respect to the index $\nu$. If, on one hand, Einstein's equations contain this conservation law, on the other hand, the latter also contains Einstein's equations to which the energy-momentum tensor under consideration refers. Thus, the equations of the gravitational field contain and are contained by the equations for the matter which produces this field. From a mathematical point of view, such a correspondence is represented by the direct computation of the covariant derivative with respect to $\nu$ of the left hand side of Einstein's equations. Indeed, by taking into account the identity $R_{\mu \nu}^{i \nu}=\frac{1}{2} \frac{\partial R}{\partial x_{\mu}}$, zero is obtained and so the equality is respected. As a consequence, the distribution and motion of the matter producing the gravitational field can not be assigned arbitrarily, but it must be computed by solving Einstein's equation under given initial conditions at the same time as we find the field produced by the matter.

Unlike the gravitational field equations, Maxwell's equations contain only the the charge conservation law (i.e. the equation of conservation of the total charge or continuity equation), but not the equations of motion of the charges themselves. For this reason, the distribution and motion of the charges can be assigned arbitrarily, as long as the total charge is constant. The assignment of this charge distribution then determines, through Maxwell's equations, the electromagnetic field produced by the charges themselves.

At the present time it is usually taken for granted that the distribution of matter in the Universe must be homogeneous and isotropic, at least in the early stage, as indicated by isotropy of cosmic microwave background radiation (CMB), and even now at large scales. Consequently, the most general isotropic and homogeneous metric can be assumed. It goes by the name of Friedman-Robertson-Walker (FRW) metric and corresponding invariant distance (or line element) reads:

$$
d s^{2}=g_{\mu \nu} d x^{\mu} d x^{\nu}=d t^{2}-a^{2}(t)\left[f(r) d r^{2}+r^{2} d \Omega\right],
$$

where the function $f(r)=1 /\left(1-k r^{2}\right)$ describes the 3D space of constant curvature $k$. Particularly, it draws a 3D hyperboloid (open Universe), a 3D sphere (closed Universe) and a flat 3D space when $k=+1,-1,0$, respectively. The function $d \Omega^{2}=d \theta^{2}+\sin ^{2} \theta d \phi^{2}$ gives a measure of angle on the sky of the observer with the angles $\phi$ and $\theta$ the usual azimuthal and polar angles of spherical coordinates, with $\theta \in[0, \pi], \phi \in[0,2 \pi)$. Finally, $a(t)$ represents the scale factor which describes the observed expansion of the Universe through redshift analysis.

A simplifying assumption (compatible with the isotropy and homogeneity of the universe) is that the matter and energy content of the universe can be described in terms of a perfect fluid. Hence, the energy momentum tensor in the rest frame of the perfect fluid and for an arbitrary 
four-velocity $v^{\mu}$ of the perfect fluid take the two following forms:

$$
T_{\mu \nu}=\left(\begin{array}{cccc}
+\rho & 0 & 0 & 0 \\
0 & p & 0 & 0 \\
0 & 0 & p & 0 \\
0 & 0 & 0 & p
\end{array}\right) \quad(\text { rest }), \quad T_{\mu \nu}=(\rho+p) v_{\mu} v_{\nu}-p g_{\mu \nu} \text { (in motion), }
$$

where $\rho$ and $p$ are, respectively, the energy density and the isotropic pressure of the fluid.

The evolution of the scale factor $a(t)$, and so the dynamics of the all Universe, is obtained by solving Friedman's equation:

$$
\left(\frac{\dot{a}(t)}{a(t)}\right)^{2} \equiv H(t)^{2}=\frac{8 \pi G_{N}}{3} \sum_{i} \rho_{i}(t)-\frac{k}{a(t)^{2}},
$$

along with the acceleration equation:

$$
\frac{\ddot{a}(t)}{a(t)}=\dot{H}(t)+H(t)^{2}=-\frac{4 \pi G_{N}}{3} \sum_{i}\left(\rho_{i}(t)+3 p_{i}(t)\right),
$$

which in turn are easily obtained by the equations of General Relativity for FRW metric. Here the index $i$ is for the $i$ th component of the energy-matter content of the Universe while $\dot{a}$ and $\ddot{a}$ denote the first and second time derivative of $a$, respectively.

The overall value of the scale factor $a(t)$ is arbitrary, only relative changes are measurable. In the previous two equations we have also introduced the Hubble parameter:

$$
H(t)=\frac{\dot{a}(t)}{a(t)}
$$

that governs the local expansion of the Universe according to Hubble's law:

$$
v=H(t) d
$$

where $v$ is the recession velocity and $d$ is the physical distance. In order to clarify the meaning of physical distance in this context, we have displayed in Figure 2.6 the space in terms of a grid which expands uniformly as time evolves. Even though the points on the grid maintain their coordinates, and therefore the so-called comoving distance does not change with time, the physical distance does change. In fact, it is proportional to the time dependent scale factor.

The present value of Hubble parameter (named Hubble constant) is parameterized as:

$$
H_{o} \equiv H\left(t_{o}\right)=100 h \mathrm{Km} \mathrm{s}^{-1} \mathrm{Mpc}^{-1},
$$

where the Planck satellite has set the reduced Hubble constant $h$ to the value $0.6727 \pm 0.066$, according to its latest data (2015) [80]. Note that the subscript "o" of each coming physical quantity refers to present-day values of such a quantity (unless otherwise indicated).

Directly from Friedman's equation (2.8) we can see that Universe is flat $(k=0)$ when the energy density equals the critical energy density $\rho_{c}$, which is given by the coming formula:

$$
\rho_{c}(t)=\frac{3 H(t)^{2}}{8 \pi G_{N}} .
$$




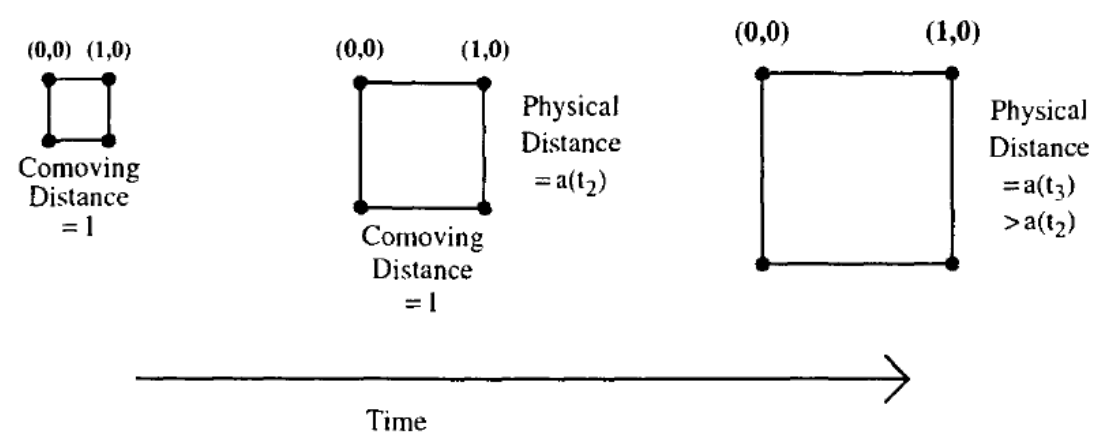

FIGURE 2.6: Comoving distance on an hypothetical grid does not change with time (with the expansion of the Universe) [66]. Physical distance is proportional to the comoving distance times the scale factor.

By using the Friedman's equation (2.8) and the acceleration equation (2.9) is possible to derive the continuity equation:

$$
\dot{\rho}_{i}(t)+3 H(t)\left(\rho_{i}(t)+p_{i}(t)\right)=0
$$

which is nothing than the covariant conservation of the energy-momentum tensor.

The connection between the energy density $\rho_{i}$ and the isotropic pressure $p_{i}$ for each species existing in the Universe gives the equation of state:

$$
p_{i}(t)=w_{i} \rho_{i}(t)
$$

The parameter $w_{i}$ determines the matter properties. Particularly, non-relativistic matter has $w_{m}=0$ whereas relativistic matter has $w_{r}=1 / 3$. Finally, the type of matter (or vacuum) that can be described by an energy component with negative pressure has $w_{\Lambda}=-1$.

The expansion of the Universe means that the scale factor $a(t)$ has been increasing since the earliest times after Big Bang, the explosion from which the expansion started. The first observational evidence for this was Hubble's detection of a cosmological redshift of the light emitted by distance galaxies. For the wavelength $\lambda_{e}$ and the scale factor $a_{e}$ in emission and the wavelength $\lambda_{o}$ and the scale factor $a_{o}$ in receiving (at the present-day), the redshift parameter $z$ is defined to be:

$$
1+z \equiv \frac{\lambda_{o}}{\lambda_{e}}=\frac{a_{o}}{a_{e}}
$$

From the continuity equation (2.14) it is possible to obtain the following dependence of the energy density on the redshift parameter:

$$
\rho_{i}(z)=\rho_{i_{o}}(1+z)^{3\left(1+w_{i}\right)}
$$

where $\rho_{i_{o}} \equiv \rho_{i}(0)$. The energy density of non-relativistic matter decreases like $(1+z)^{3}$ because of the dilution of the number density with the expansion of the Universe. On the contrary, the energy density of relativistic matter decreases by an additional factor $(1+z)$ because of the energy redshift in an expanding Universe. Finally, the energy component with negative pressure (equivalent to an intrinsic energy of the vacuum) does not dilute away with the changing volume. Note that such a component is known as cosmological constant of the Universe. 
Both the Friedman's equation (2.8) and the acceleration equation (2.9) can be rewritten by using the critical energy density $\rho_{c}(z)$, the energy density as a function of the redshift parameter $z$ and the density parameter:

$$
\Omega_{i}(z)=\frac{\rho_{i}(z)}{\rho_{c}(z)}=\Omega_{i_{o}}(1+z)^{3\left(1+w_{i}\right)}, \quad \Omega_{i_{o}} \equiv \Omega_{i}(0)
$$

that gives the energy density of the $i$-th component in units of the critical energy density. The two equations, respectively, become:

$$
\begin{gathered}
1=\Omega_{t o t}(z)-\frac{k}{a^{2} H^{2}}=\frac{H_{o}^{2}}{H^{2}}\left[\Omega_{r_{o}}(1+z)^{4}+\Omega_{m_{o}}(1+z)^{3}+\Omega_{\Lambda}-\frac{k}{a_{o}^{2} H_{o}^{2}}(1+z)^{2}\right] \\
\frac{\ddot{a}}{a H^{2}}=-\frac{1}{2} \sum_{i} \Omega_{i}(z)\left(1+3 w_{i}\right)=-\frac{1}{2} \sum_{i} \Omega_{i_{o}}(1+z)^{3\left(1+w_{i}\right)}\left(1+3 w_{i}\right)
\end{gathered}
$$

where $\Omega_{\text {tot }}(z) \equiv \sum_{i} \Omega_{i}(z)=\Omega_{r}(z)+\Omega_{m}(z)+\Omega_{\Lambda}(z)$. Cosmological observations suggest that the Universe is spatially flat. Furthermore, since the radiation density is negligible today, the current expression of the total energy density becomes:

$$
\Omega_{t o t_{o}} \simeq \Omega_{m_{o}}+\Omega_{\Lambda_{o}} \simeq 1
$$

Note that the value $\Omega_{\text {tot }_{o}}=1$ in Equation (2.19) is equivalent to having a flat Universe $(k=0)$. For simplicity and brevity, we suppress the explicit dependence of $a$ on both $t$ and $z$.

In the last part of this subsection we present the solutions of the cosmological equations for several special cases which were all realized in the history of the Universe at different stage of its evolution. In the following it is always assumed that the 3D space is flat $(k=0)$.

Let us start considering the case of non-relativistic matter. Its equation of state $\left(p_{m}=0\right)$ along with Equation (2.14) give the later easy equation for the evolution of its energy density:

$$
\dot{\rho}_{m}+3 H \rho_{m}=0 \text {. }
$$

The solution of this equation, $\rho_{m} \sim 1 / a^{3}$, is straightforward. Indeed, it simply describes the dilution of the number density of massive particles at rest. The time dependence of the cosmological scale factor, given by Friedman's equation, reads therefore:

$$
a(t) \sim t^{2 / 3}
$$

in the non-relativistic regime. Consequently, the Hubble constant decreases as time evolves, $H(t)=2 / 3 t$, and we deal with a decelerating Universe. According to this model, the age of the Universe would be $t=2 / 3 H_{o}=9.26 \times 10^{9}$ years. This matter-dominated Universe is the so-called Einstein-de Sitter Universe.

The equation of state for relativistic matter $\left(p_{r}=\rho_{r} / 3\right)$ gives, instead, the following continuity equation:

$$
\dot{\rho}_{r}=-4 H \rho_{r} .
$$


Thus the energy density $\rho_{r}$ drops as $\rho_{r} \sim 1 / a^{4}$ and the scale factor, again given by Friedman's equation, rises with time as:

$$
a(t) \sim t^{1 / 2}
$$

Now the Hubble constant decreases as time elapses according to $H(t)=1 / 2 t$, so the radiationdominated Universe exhibits an decelerated expansion as the matter-dominated Universe does. The age of the Universe in this model would be $t=1 / 2 H_{o}=6.94 \times 10^{9}$ years.

By comparing the energy density of relativistic particles with the one of non-relativistic particles, we can see that the former drops one power of the scale factor $a$ faster than the latter. Actually, we should not be surprised from this difference because of their different dilution of the number density. In fact, whereas the relativistic particles dilutes the number density as the volume $\left(1 / a^{3}\right)$ and the redshift of the particle momentum $(1 / a)$, the non-relativistic particles just dilute as the volume. It is also interesting to note that these different velocities of dilution can clarify why in the history of the Universe the relativistic matter dominated epoch occurs before the non-relativistic matter one. At the end, let us point out that pressure of relativistic matter does not help to neutralize the deceleration due to gravity, as one might expect. The reason is that only a pressure gradient, missing in a homogeneous Universe, can induce forces.

Finally, in the vacuum regime the equation of state $\left(p_{\Lambda}=-\rho_{\Lambda}\right)$ yields the next continuity equation:

$$
\dot{\rho}_{\Lambda}=0
$$

It is now clear that the vacuum energy density remains constant during the cosmological expansion, as already mentioned. The scale factor in this case rises exponentially:

$$
a(t) \sim e^{H t}
$$

and the age of the Universe turns to be infinite.

The current standard model of the Universe (called $\Lambda \mathrm{CDM}$ ) states that the Universe is a flat Universe containing matter and radiation and having a non-zero cosmological constant. Although a cosmological constant is the mathematically simplest, and perhaps the physically simplest, theoretical explanation for the accelerating universe, other explanations for the present acceleration exist. For instance, Dark Energy [85], a new form of energy driving the acceleration, and modified gravity [86], a modification of GR that leads to the accelerated expansion.

In Table 2.2 the parameters of the $\Lambda$ CDM model are summarized. These days it is clear that we are currently dominated by the cosmological constant $\Lambda$ by a factor of three. This means that we are now in a phase of exponential growth. Before (about $z \gtrsim 0.5$ ), the Universe was dominated by cold matter, and even before (about $z \gtrsim 3200$ ) the Universe was, instead, dominated by radiation.

\section{Inflation}

In 1980s the physicists Alan Guth and Andrei Linde introduced the inflationary epoch in cosmology to explain, first, why some of the initial conditions of the Universe had to be fine-tuned to very "special" values so as to lead to the observed cosmological parameters. Second, why a small deviation from these values would have had massive effects on the nature of the Universe at the current time. In doing so, they basically had to deal with the "homogeneity problem" 


\begin{tabular}{lcc}
\hline Parameter & symbol & value \\
\hline Age of the Universe & $t_{o}$ & $13.807 \mathrm{Gyr}$ \\
Hubble's constant & $H_{o}$ & $67.51 \mathrm{Km} / \mathrm{s} / \mathrm{Mpc}$ \\
Baryon density & $\Omega_{b_{o}}$ & 0.04919 \\
Cold dark matter density & $\Omega_{C D M_{o}}$ & 0.2636 \\
Cosmological constant & $\Omega_{\Lambda_{o}}$ & 0.6879 \\
Radshift of radiation/matter equality & $z_{e q}$ & $3382 \pm 32$ \\
Critical density & $\rho_{c_{o}}$ & $8.51 \times 10^{-30} \mathrm{~g} / \mathrm{cm}^{3}$ \\
Total matter density & $\Omega_{m_{o}}$ & 0.3121 \\
Total radiation density (photons and neutrinos) & $\Omega_{r_{o}}$ & $5.46 \times 10^{-5}$ \\
\hline
\end{tabular}

TABle 2.2: Cosmological constants derived from Planck 2015 data [80].

and the "flatness problem", two problems of the traditional big bang cosmology connected to the initial conditions of the Universe.

The "homogeneity problem" concerns the existence of nearly the same temperature of the $\mathrm{CMB}$ radiation even in regions of the sky which cannot have been in casual connection at the time of emission, namely in regions where the physical interactions cannot cause such a homogeneity of the temperature. The "flatness problem", instead, affects the observation that the current total density of the Universe is so close to the value of the critical density, and therefore, that the Universe is so unbelievably flat. The current nearly flat condition is, indeed, definitely a special case of all the possibilities from very positively-curved to very negatively-curved.

Inflation can solve both of these problems by assuming that some scalar field (inflaton) in the very early Universe slowly changed its expansion rate thanks to an effective potential which left the scalar temporarily at a non-minimum. In fact, if this potential energy density took a nearly constant value during this "slow-roll" phase and at this time it was able to dominate the energy density of the Universe, it would act as a true cosmological constant, i.e. as a superluminar exponential expansion. This expansion would create causal connected regions which would move outside the horizon of each other. According to the inflationary theory, the phase would stop when the inflaton rolled down to the true minimum of the potential. The energy released by the subsequent slowdown around the minimum should have refilled the Universe with particles, radiation and an enormous amount of entropy. This epoch is known as "reheating epoch" and, consequently, the equilibrium temperature of the thermal plasma is named reheating temperature $\left(T_{R}\right)$. After inflation, the Universe is described by $\Lambda$ CDM.

Inflation theory is not a mandatory ingredient in the modern cosmology but it is nowadays the better explanation of the above-mentioned problems, for this reason it has a prominent place in theoretical cosmology. In closing, let us point out that an additional virtue of inflation is that it can explain the origin of the small primordial fluctuations observed in the CMB radiation.

\section{Big Bang Nucleosynthesis}

The Big Bang Nucleosynthesis (BBN), also known as primordial nucleosynthesis, represents an era in the history of the Universe that is thought to have lasted from about a second until a few minutes after the Big Bang, the explosion from which the Universe started expanding. It is a non-equilibrium process taking place in an expanding, radiation-dominated plasma with high entropy ( $\sim 10^{9}$ photons per baryon) and a large number of free neutrons. During this process, protons and neutrons could combine together to form a multitude of light nuclei, which 
synthesized after in the light elements ${ }^{1} \mathrm{H},{ }^{3} \mathrm{H}, \mathrm{D},{ }^{3} \mathrm{He},{ }^{4} \mathrm{He}$ and ${ }^{7} \mathrm{Li}$ at the end of the "first three minutes". The predictions of the abundances of these elements are in good overall agreement with the primordial abundances inferred from observational data, thus validating the standard hot Big Bang cosmology. These results are particularly significant since these values cover nine orders of magnitude by ranging from ${ }^{4} \mathrm{He} / \mathrm{H} \sim 0.08$ to ${ }^{7} \mathrm{Li} / \mathrm{H} \sim 10^{-10}$.

Immediately ( $\ll 1 \mathrm{sec}$ ) after the beginning of BBN, the Universe was a hot $(\gg 1 \mathrm{MeV})$, rapidly expanding plasma, with most of its energy in radiation and relativistic particles. There were roughly equal numbers of electrons, positrons, neutrinos and antineutrinos (of each species), and photons. Nucleons (protons and neutrons) were instead outnumbered, approximately, by more than a billion to one. In addition, there were essentially no composite nuclei, and weak processes like $\nu+n \leftrightarrow p+e^{-}$maintained the ratio of neutrons-to-protons number density at its thermal equilibrium value:

$$
\frac{n_{n}}{n_{p}}=e^{-\frac{m_{n}-m_{p}}{T_{e q}}} \simeq 1
$$

where $m_{n}\left(m_{p}\right)$ is the neutron (proton) mass and $T_{e q}(\sim 1 \mathrm{MeV})$ is for the equilibrium temperature. In Equation (2.28) we have left out the chemical potentials $\mu_{n}$ and $\mu_{p}$ since $\left(\mu_{n}-\mu_{p}\right) / T_{e q}$ is negligible with respect to $\left(m_{n}-m_{p}\right) / T_{e q}$ for the temperature of interest, and thus:

$$
e^{\frac{-\left(\mu_{n}-\mu_{p}\right)-\left(m_{n}-m_{p}\right)}{T_{e q}}} \simeq e^{-\frac{m_{n}-m_{p}}{T_{e q}}} .
$$

At about one second, the temperature had dropped to around $1 \mathrm{MeV}$. The weak processes became ineffective and so, the neutron-to-proton ratio leveled off at about $1 / 6$. This moment is known as nucleons freeze-out. Note that the number of relativistic particle species (e.g. the number of light neutrino species) and the baryon-to-photon ratio $\left(\eta \equiv n_{b} / n_{\gamma}\right)$ are important to determine the freeze-out time. Growing amounts of $\mathrm{D},{ }^{3} \mathrm{He},{ }^{3} \mathrm{H}$, and ${ }^{4} \mathrm{He}$ were present in amounts dictated by the nuclear statistical equilibrium. The processes maintaining this equilibrium, due to the decreasing temperatures and the densities, slowed down until these precesses are no longer efficient.

After five minutes, most neutrons were in ${ }^{4} \mathrm{He}$ nuclei, and most protons remained free. At this time neutron-to-proton ratio slightly decreased to $1 / 7$. Much smaller amounts of $\mathrm{D},{ }^{3} \mathrm{He}$, and ${ }^{7} \mathrm{Li}$ were synthesized, but their low density and the bottleneck, namely the absence of a stable nucleus with 8 or 5 nucleons, made sure that Big Bang Nucleosynthesis did not produce elements heavier than beryllium (Be). The elemental composition of the Universe subsequently remained unchanged until the formation of the first stars several billion years later. The yields of primordial nucleosynthesis, with $2 \sigma$ theoretical errors, are shown as a function of the baryon density in Figure 2.7.

\section{Cosmic Microwave Background}

Perhaps the most conclusive piece of evidence for the Big Bang is the existence of the CMB radiation. We have already discussed such a radiation in Section 2.2 in the context of the DM evidences.

The degree of anisotropy of the CMB is very high (roughly about one part in a thousand) but not perfect. As already mentioned, there are small fluctuations in the temperature across the sky at $10^{-5}$ level, the so-called microwave background temperature anisotropies. These 


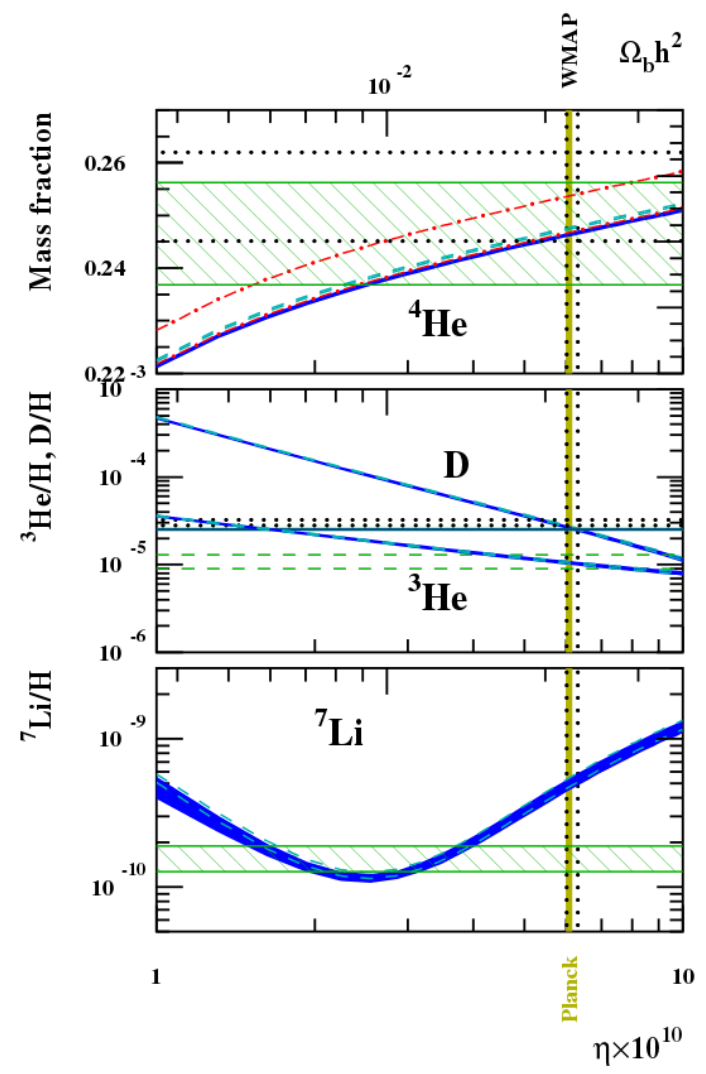

FIGURE 2.7: BBN predictions of the light elements abundances. All bands show the $2 \sigma$ uncertainties. The vertical areas correspond $i$ ) to the WMAP (dot, black) and ii) Planck (solid, yellow) baryonic densities. The horizontal areas (hatched green) represent the adopted observational abundances while the horizontal dotted lines correspond to those previously used [87, 88]. The (red) dash-dotted lines correspond to $Y_{p}$ calculated with $N=3.30 \pm 0.27$ derived from the CMB [89].

anisotropies are usually expressed by using a spherical harmonic expansion of the CMB sky:

$$
\frac{\Delta T}{T}(\theta, \phi)=\sum_{\ell m} a_{\ell m} Y_{\ell m}(\theta, \phi),
$$

where $\ell \in[1, \infty]$ and $m \in[-\ell, \ell]$, i.e. the index $m$ runs over $2 \ell+1$ values for each $\ell$. Such an expansion results in the CMB power spectrum $\ell(\ell+1) C_{\ell} /(2 \pi)$ in terms of multipole moments $\ell$ with:

$$
C_{\ell} \equiv\left\langle\left|a_{\ell m}\right|^{2}\right\rangle=\frac{1}{2 \ell+1} \sum_{m}\left|a_{\ell m}\right|^{2}
$$

Note that, given a cosmological model with a limited number of parameters, the best-fit values of its cosmological parameters can be obtained by simply using the observed CMB power spectrum. In particular, the today parameter region $\Omega_{\Lambda^{-}} \Omega_{m}$ for $\Lambda$ CDM (which assumes a cosmological constant $\Lambda$ and a dominant Cold Dark Matter component) is displayed in Figure 2.8. Here we can see that the observations of $\mathrm{CMB}$, supernovae (SNeIa) and baryon acoustic oscillations (BAO) overlap, and their combination suggests a flat universe with a cosmological constant density of $\Omega_{\Lambda} \simeq 0.70$ and a total matter density of $\Omega_{m} \simeq 0.30$. 


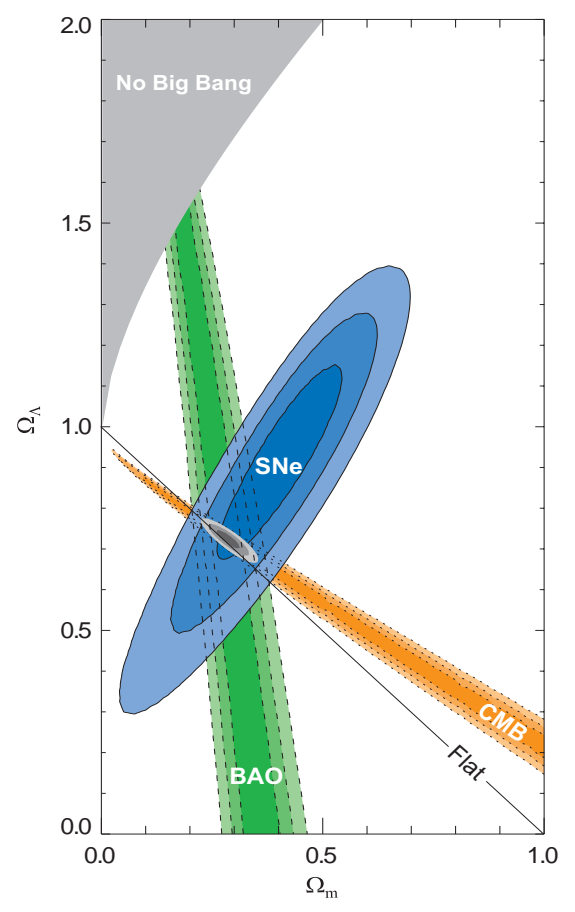

FIGURE 2.8: Observational constraints in $\Omega_{\Lambda}-\Omega_{m}$ plane. The contours represent the $68.3 \%$, 95.4\% and 99.7\% confidence level from CMB, BAO and SNeIa and their combination, with different colors corresponding to different data sets. The green contours represent BAO constraints; the orange contours correspond to constraints from CMB anisotropies. Finally, the blue contours denote constraints from supernovae and the gray one is for the values where we have no Big Bang. This figure is taken by [90].

\section{Baryogenesis}

The problem of the baryon asymmetry of the Universe is a classic problem of particle cosmology. Particle physics claims that matter and antimatter behave essentially identically, therefore the interactions between matter and antimatter are nowadays the focus of successful terrestrial experiments. On the other hand, cosmology states that the early Universe was an extremely hot, and so energetic, environment where one would expect equal numbers of baryons and antibaryons to be produced. It is remarkable that this early state of the universe deeply disagrees with what we observe today, i.e. no appreciable primordial antimatter.

The theory of primordial nucleosynthesis allows accurate predictions of the cosmological abundances of all the light elements, while requiring only that the baryon-to-photon ratio $\eta$ belongs to the range:

$$
2.6 \times 10^{-10}<\eta \equiv \frac{n_{b}-n_{\bar{b}}}{s}<6.2 \times 10^{-10},
$$

where $n_{b}\left(n_{\bar{b}}\right)$ and $s$ denote the number density of baryons (antibaryons) and the entropy density, respectively.

As pointed out by Sakharov, a small baryon asymmetry may have been produced in the early Universe if three necessary conditions are satisfied: i) baryon number (B) violation; ii) violation of $\mathrm{C}$ (charge conjugation symmetry) and $\mathrm{CP}$ (the composition of parity and $\mathrm{C}$ ) and iii) departure from thermal equilibrium. The first condition should be clear since, starting from a baryon symmetric universe with $\eta=0$, baryon number violation must take place in order to 
evolve into a universe in which $\eta$ does not vanish. The second Sakharov criterion is required because, if $\mathrm{C}$ and $\mathrm{CP}$ are exact symmetries, one can prove that the total rate for any process producing an excess of baryons is equal to the rate of the complementary process producing an excess of antibaryons, and so no net baryon number can be created. At last, to explain the third criterion, one can calculate the equilibrium average of $\mathrm{B}$ at a temperature $T=1 / \beta$ :

$$
\begin{aligned}
& \langle B\rangle_{T}=\operatorname{Tr}\left[e^{-\beta H} B\right]=\operatorname{Tr}\left[(C P T)(C P T)^{-1} e^{-\beta H} B\right] \\
& \quad=\operatorname{Tr}\left[e^{-\beta H}(C P T)^{-1} B(C P T)\right]=\operatorname{Tr}\left[-e^{-\beta H} B\right]=-\langle B\rangle_{T},
\end{aligned}
$$

where we have used that the Hamiltonian $\mathrm{H}$ commutes with CPT. Thus, we have $\langle B\rangle_{T}=0$ in equilibrium, and so there is no generation of net baryon number.

These ingredients are all present in the Standard Model. However, no SM mechanism generating a large enough baryon asymmetry has been found. Baryogenesis therefore requires new physics beyond SM in (at least) two ways: It must introduce new sources of CP violation and it must either provide a departure from thermal equilibrium in addition to the electroweak phase transition (EWPT) or modify the EWPT itself.

Several models have been proposed for Baryogenesis so far. The currently favored one goes under the name Baryogenesis via Thermal Leptogenesis [91]. In this model baryon asymmetry is generated from a lepton asymmetry. In fact, a non-vanishing lepton number L can be converted into a non-vanishing baryon number via non-perturbative sphaleron processes. The required lepton number L can be generated in $\mathrm{CP}$ violating out-of-equilibrium decay of heavy righthanded Majorana neutrinos. Since heavy Majorana neutrinos via Seesaw mechanism can also justify the tiny masses of the SM neutrinos, this mechanism of baryon asymmetry production is strongly supported by the recent observation of non-vanishing neutrino masses.

Let us highlight that this mechanism demands high reheating temperatures in the early Universe $\left(T_{R} \gtrsim 10^{9} \mathrm{GeV}[92,93]\right)$ in order to achieve the observed baryon asymmetry.

\subsection{Dark Matter production}

In this section we review three different mechanism of DM production: WIMP, SuperWIMP and Freeze-in. Physicists have indeed proposed some DM candidates until now - such as WIMP (e.g. neutralino), gravitino, axions - arising from different mechanisms of production. In principle, some of these candidates, and so their mechanisms, could even coexist since there is no reason why their contributions to DM should be at the same level. We point out here that, although SuperWIMP and Freeze-in are two completely different and independent mechanisms, they are related to each other by the same decay channel into DM of the same particle $(\Sigma)$ in and out of thermal equilibrium.

Both SuperWIMP and Freeze-in are the most relevant mechanisms for this thesis since they are directly used along with the minimal decaying DM model of Chapter 1 in order to generate a scalar field capable of generating DM in the right amount. In particular, we employ this setup, first, to study the correlation between DM Indirect Detection and CMS signals in Chapter 4 and, second, to account for the recently observed $3.55 \mathrm{keV}$ photon line in Chapter 5 .

In the interest of clarity, in the last section of this chapter we analyze in more detail the SuperWIMP and Freeze-in mechanisms in connection with the minimal model of DM decaying, mentioned above. 


\section{WIMP}

One of the most popular mechanisms of DM production is the WIMP mechanism. In fact, this mechanism generates one of the best DM candidates, the so-called Weakly Interacting Massive Particles (WIMPs) category (see e.g. [69] for review). Particles in this category are thermal relic and can achieve the appropriate value of the DM relic density by interacting with the SM sector with roughly electroweak strength, like ordinary neutrinos.

In this scenario WIMP DM is made of beyond SM (BSM) particles, which are stable, initially in thermodynamic equilibrium with the surrounding hot plasma. At the time when their interaction rate drops below the expansion rate of the Universe, WIMPs decouple from the plasma as a non-relativistic species. The main difference with neutrino DM lies in the mass of WIMPs, which should be large enough such that these particles decouple already non-relativistic.

For a non-relativistic particle at decoupling, the ratio of number density to entropy density $(Y=n / s)$ remains frozen, in other words the thermal relic freezes-out. The evolution of the number density $n$ of a generic species $\chi$ can be evaluated via the Boltzmann equation:

$$
\frac{d n}{d t}+3 H n=-\left\langle\sigma_{a n n} v\right\rangle\left[n^{2}-n_{e q}^{2}\right] .
$$

In this equation $3 H n$ describes the dilution of the number density due to the expansion of the Universe while $\left\langle\sigma_{a n n} v\right\rangle$ the thermal average of the annihilation cross section times velocity and it can be parameterized through the next non-relativistic expansion in powers of $v^{2}$ :

$$
\left\langle\sigma_{a n n} v\right\rangle=a+b\left\langle v^{2}\right\rangle+O\left(\left\langle v^{4}\right\rangle\right) \simeq a+\frac{6 b}{x} \quad \text { with } \quad x=\frac{m_{\chi}}{T} .
$$

Finally, $n_{e q}$ is for the number density at thermal equilibrium when the temperature is $T$. For non-relativistic particles such an equilibrium number density is given by the Maxwell Boltzmann approximation:

$$
n_{e q}=g\left(\frac{m_{\chi}}{2 \pi}\right)^{3 / 2} e^{-\frac{m_{\chi}}{T}}
$$

where $m_{\chi}$ is the WIMP mass and $g$ is for the number of degree of freedom of WIMP.

The Boltzmann equation can be solved by integrating it in a region long before the WIMP freeze out and long after it, and then by matching these two solutions. Due to the lack of time we skip the calculation details and give immediately the today relic density for the WIMP $\chi$ :

$$
\Omega_{\chi} h^{2} \simeq \frac{3 \times 10^{-27} \mathrm{~cm}^{3} \mathrm{~s}^{-1}}{\left\langle\sigma_{a n n} v\right\rangle} .
$$

This result shows that the relic abundance of a non-relativistic decoupled species strictly depends on the annihilation cross section at freeze-out. Moreover, this result for weak cross-sections gives the right order of magnitude of the DM density (and a temperature $T_{f . o .} \simeq m / 20$ at freeze-out for a WIMP of mass $m$ ). This coincidence of scales - the relic density and the weak force interaction scale - is sometimes referred to as the "WIMP miracle" and was part of the motivation to adopt the WIMP as the leading candidate for cosmological dark matter.

For the purposes of completeness it is worth to note that the annihilation cross-section, for a particle of given mass, has a maximum, imposed by the partial wave unitarity of the S-matrix, $\left\langle\sigma_{a n n} v\right\rangle_{\max } \sim 1 / m_{\chi}^{2}$ [94]. The above result for $\Omega_{\chi} h^{2}$ with the requirement $\Omega_{m_{o}} h^{2} \lesssim 1$ thus 
provides with the "unitarity bound" on the DM mass:

$$
m_{D M} \lesssim 340 \mathrm{TeV}
$$

The most studied WIMP DM candidate is the lightest Neutralino [25] of the MSSM. Within the particle content of the MSSM, and over a large range of parameters, the LSP can be either the lightest Neutralino, the lightest Sneutrino or the lightest Stau. Among these, only the lightest Neutralino is still allowed by present experiments as a viable DM candidate $[1,69,95]$.

The above-discussed standard computation of the thermal relic abundance has three exceptions $[96,97]$. They take place for WIMPs lying near a mass threshold, for annihilations near to a pole in the cross section, or in presence of coannihilations. Particularly, the latter occurs when a particle that shares a quantum number with the WIMP, is nearly degenerate in mass with it. If the mass gap is low enough (roughly $\lesssim 10 \%$ ) the coannihilation reactions, involving WIMP particles, can control the WIMP abundance and lower or enhance it.

The interest for this class of candidates is due to their potential relation to the electroweak symmetry breaking, which is being tested at LHC. The WIMP searches are also important scientific goals of many other existing laboratory experiments, for instance the dozens experiments conducted to detect WIMPs in the Galaxy halo by testing their interaction with nucleons.

\section{SuperWIMP}

Another mechanism of DM production is known under the name of SuperWIMP mechanism. As a result, the corresponding DM candidate class is named SuperWIMPs [98]. SuperWIMPs do not experience the thermal history discussed above; on the contrary they are typically generated in the late decay of other particles that have earlier decoupled from the thermal bath (SuperWIMP mechanism). In most cases the SuperWIMP thermal production is negligible.

In this scenario, the relic density of a SuperWIMP $X$ (DM candidate), which is produced in the decay of the more massive particle $\Sigma$, is simply given by:

$$
\Omega_{X}=\frac{m_{X}}{m_{\Sigma}} \Omega_{\Sigma}
$$

where $\Omega_{X}\left(\Omega_{\Sigma}\right)$ and $m_{X}\left(m_{\Sigma}\right)$ represent, respectively, the relic density and the mass of $X(\Sigma)$. Hence, the SuperWIMP inherits in general the relic density of the parent particle $\Sigma$. If the parent particle is e.g. a WIMP, then this can give quite naturally the correct relic density to the SuperWIMP particle, despite its very weak interaction with the visible world and its often tiny annihilation cross-section. Typical collider signatures of this SuperWIMP scenario are these parent particles, which are often quite long-lived and might lead to distinct signatures in the detector, such as displaced decays and metastable tracks. In Chapter 4 we investigate such a scenario and its signatures for the minimal model of DM decaying, introduced in Chapter 1.

If the decay of parent particles takes place during BBN, the hadronic and electromagnetic energy released in the decay can destroy the successful predictions of the standard BBN scenario (see e.g. [99,100]). A good recent review about BBN bounds can be found in [101].

\section{Freeze-in}

The Freeze-in mechanism of DM production $[102,103]$ provides another simple and intriguing alternative to the WIMP paradigm. Let us assume that at temperature $T$ there is a set of bath 


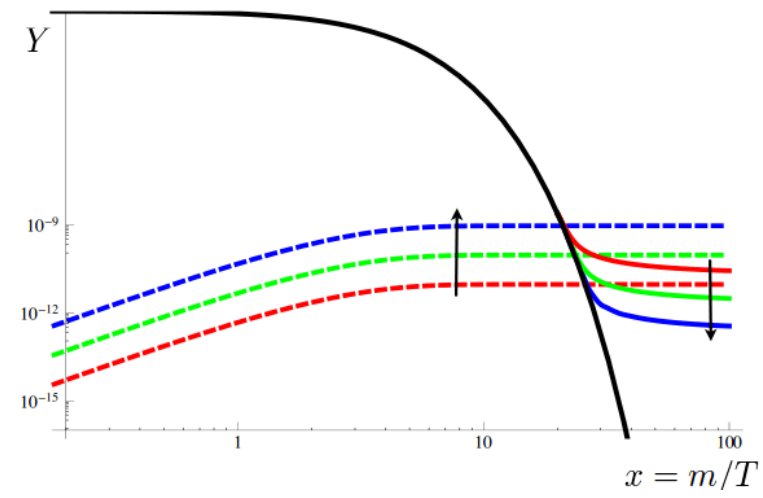

FiguRE 2.9: Log-Log plot of the evolution of the relic yields for conventional freeze-out (solid coloured) and freeze-in via a Yukawa interaction (dashed coloured) as a function of $x$. The black solid line denotes the yield in equilibrium, while the arrows indicate the effect of increasing coupling strength for the two processes.

particles that are in thermal equilibrium and some other long-lived particle $X$ (FIMP), having interactions with the bath that are so feeble that $X$ is thermally decoupled from the plasma. In this scenario, we also make the assumption that the earlier history of the Universe makes the abundance of $X$ negligibly small. Although the interactions with the bath particles are feeble, they lead to some $X$ production and, for renormalizable interactions, the dominant production of $X$ occurs as $T$ drops below the mass of $X$. The abundance of $X$ then freezes-in with a yield that increases with the interaction strength of $X$ with the bath $\Sigma$. Another population of $X$ is generated by the decay of the more massive particle $\Sigma$ in thermal equilibrium. Note that this production is important, since otherwise the relic density of $X$ would grow even after $\Sigma$ became non-relativistic, i.e. when the temperature drops below the mass of $\Sigma$. The relic density of the FIMP $X$ (DM candidate) is given by [102]:

$$
\Omega_{X}=\frac{1.09 \times 10^{27} g_{\Sigma}}{g_{*}^{3 / 2}} \frac{m_{X} \Gamma_{\Sigma}}{m_{\Sigma}^{2}}
$$

where $g_{\Sigma}$ are the number of internal degree of freedom of $\Sigma$ while $g_{*}$ the number of relativistic degree of freedom of the primordial plasma at the time of DM production. Finally, $\Gamma_{\Sigma}$ stands for the $\Sigma$ decay rate.

Freeze-in mechanism can be viewed as the opposite process to freeze-out, in the sense that as the temperature drops below the mass of the relevant particle, the DM is either heading away from (freeze-out) or towards (freeze-in) thermal equilibrium. In fact, if freeze-out begins with a important initial DM abundance, and reducing the interaction strength helps to maintain this large abundance, freeze-in begins with a negligible DM thermal number density, but increasing the interaction strength increases the production from the thermal bath. These two opposite trends as a function of temperature of the DM abundance $(x=m / T)$ are shown in Figure 2.9.

\subsection{Gravitino as Dark Matter candidate}

The non-WIMP category provide a long list of possible particles. Because of the lack of time and because of the analysis of Chapter 3, we only describe the gravitino superparticle as DM candidate in the following part of this section. 
Gravitino was the first supersymmetric DM candidate ever proposed. As already stated in Chapter 1, gravitino DM can suffer from the so-called "gravitino problem" if it decays during or after Big Bang Nucleosynthesis. Such a problem can be avoided if gravitino is the LSP. In this case, indeed, it can be a very good candidate, either as a stable particle or as a metastable particle with a lifetime longer than the age of the Universe.

In the early Universe, if gravitino was in thermal equilibrium then its relic density would dilute away by inflation, thus avoiding the re-collapse of the Universe. Moreover, in order that gravitino is a CDM candidate, it must be heavy to interact so weakly not to be in equilibrium and therefore have a smaller number density than the equilibrium one.

The right amount of gravitino CDM can be thermally generated through scatterings in the primordial plasma after reheating and its thermal relic density can be computed via a Boltzmann equation:

$$
\frac{d n_{3 / 2}}{d t}+3 H n_{3 / 2}=C_{3 / 2}
$$

where the term proportional to $H$ accounts for the dilution by the cosmic expansion while the collision term $C_{3 / 2}$ describes the production of gravitinos and is given by integrating the thermal gravitino production rate. In particular, if we assume goldstino component dominates the production, the relic energy density, obtained from solving Equation (2.40), reads as follows:

$$
\Omega_{3 / 2} h^{2} \sim 0.3\left(\frac{m_{3 / 2}}{1 \mathrm{GeV}}\right)^{-1}\left(\frac{T_{R}}{10^{10} \mathrm{GeV}}\right) \sum_{i} c_{i}\left(\frac{M_{i}}{100 \mathrm{GeV}}\right)^{2} .
$$

The coefficients $c_{i}$ are of order one, the corresponding $M_{i}$ denote the three gaugino masses at EW temperature and, finally, $T_{R}$ is the reheating temperature after inflation.

From Equation (2.41) we can see that DM density is set by the sparticle masses and the reheating temperature $T_{R}$. The freedom of choosing this temperature allows to obtain the right DM density. A large reheating temperature around $10^{10} \mathrm{GeV}$ is only possible for gravitino masses above the range 1-10 GeV. Moreover, an upper bound on $T_{R}$ is obtained to avoid overclosure.

If gravitino is not the LSP, it decays $\mathrm{s}$ into lighter particles with a lifetime approximately given by:

$$
\tau_{3 / 2} \sim 3.2 \text { years }\left(\frac{100 \mathrm{GeV}}{m_{3 / 2}}\right)^{3},
$$

This late decay is clearly in conflict with BBN and so implies an upper bound on the reheating temperature to ensure that the BBN predictions are not significantly altered: $T_{R} \lesssim 10^{5} \mathrm{GeV}$. This low value for $T_{R}$ is, however, not compatible with the value for $T_{R}$ favored by thermal leptogenesis and the motivation of a heavy gravitino DM. This problem can be bypassed in two different ways. First way consists of making gravitino mass as heavy as $O(100 \mathrm{TeV})$ or even heavier so that gravitino could decay early enough and thus avoid the problem. Second way instead is that gravitino is the LSP and therefore stable. Actually, there is another way to pass this problem, namely, having a light non-WDM gravitino, but this is not our case.

If gravitino is the LSP, the decays of NLSP into a gravitino and Standard Model particles are much faster than the gravitino decays in the previous case but strongly constrained by BBN. 
The NLSP lifetime in such a case takes the form:

$$
\tau_{N L S P} \sim 9 \text { days }\left(\frac{m_{3 / 2}}{10 \mathrm{GeV}}\right)^{2}\left(\frac{150 \mathrm{GeV}}{m_{N L S P}}\right)^{5} .
$$

The NLSP is thus present during or after BBN and its late decays may spoil the predictions of standard BBN. In particular, long-lived neutralino NLSP, long-lived stau NLSP as well as long-lived stop NLSP suffer from this problem, on the contrary, NLSPs like sneutrinos do not substantially affect the BBN predictions. We remark that in Chapter 3 the decay of the longlived stop will be indeed subject to BBN constraints.

Actually, there is a more general solution of this problem involving the introduction of a small R-parity violation in the theory. Such a violation indeed causes the NLSP to decay into Standard Model particles before the onset of BBN. Because of the double suppression of the gravitino couplings to Standard Model particles by the Planck mass and the small R-parity violation, the gravitino remains very long-lived and therefore a viable candidate for CDM. In this exciting scenario we are going to investigate in the next chapter the case of stop NLSP decaying into gravitino LSP and top at LHC.

It is worth noting that another population of gravitinos is generated by the decay of the NLSP out of equilibrium through the Super-WIMP mechanism. Here, the gravitino energy density is given by the formula:

$$
\Omega_{3 / 2} h^{2}=\frac{m_{3 / 2}}{m_{N L S P}} \Omega_{N L S P} h^{2} .
$$

Note that constraints coming from BBN make the value of the NLSP energy density smaller than the present DM density and therefore make the NLSP relic density effectively negligible.

\subsection{DM production in the Mininal DM decaying model}

In order to learn if this kind of scenario is still available when the cosmological constraints on the DM abundance are taken into account, first we have to examine the possible mechanisms which allow to generate the right DM density from the couplings considered and secondly we have to discuss how the DM density constraints reduce the parameter space of the model. In our simplified setup, the interactions are encoded in the two couplings $\lambda, \lambda^{\prime}$ and assumptions on the size of these couplings is only determined by the requirement of DM viability and detection limits.

The DM abundance in the primordial plasma can be computed by solving a system of coupled Boltzmann equations for the scalar $\Sigma_{f}$ and DM $\psi$. On the other hand, $\Sigma_{f}$ also feels gauge interactions with ordinary matter which guarantee efficient annihilation and scattering processes keeping it in thermal equilibrium until it freezes-out. The evolution of number densities of $\Sigma_{f}$ and $\psi$ can then be decoupled and the main trends can be identified on purely analytical grounds (for a numerical treatment of the $\Sigma_{f}$ and $\psi$ Boltzmann equations see [8]).

According to the value of the coupling $\lambda$, different generation mechanisms can account for the DM relic density. Specifically, two main scenarios can be found. The first one is characterized by very low values of $\lambda$, below $10^{-7}$. DM is not in thermal equilibrium in the Early Universe for these low values of the coupling. However, Freeze-in and SuperWIMP generation mechanisms 
involving the decay of $\Sigma_{f}$ into DM are active ${ }^{3}$. As already stated, DM is produced by decays (and in principle also scattering [8]) of $\Sigma_{f}$ while it is in equilibrium according to the Freeze-in paradigm, whereas it is non-thermally produced by the $\Sigma_{f}$ decay after it has undergone freezeout, according to the SuperWIMP mechanism. The second scenario is instead marked by a $\lambda$ of order one. In this case DM is in thermal equilibrium in the early stages of the cosmological history and so is produced depending on the WIMP mechanism. As can be argued from Equation (1.84) of the previous chapter, in this last case Indirect Detection (ID) limits require an extreme suppression of the coupling $\lambda^{\prime}$; as a consequence this second case essentially corresponds with the standard WIMP models which have already been object of numerous studies across the literature.

Because of the low values of the coupling $\lambda$ coming into play, below we only investigate the Freeze-in and SuperWIMP regimes. Moreover, due to the long lifetime of DM, requiring at least one of the additional charged particles $\Sigma_{f}$ to be within the LHC reach constrains all couplings to be suppressed and thus also points to regions of (near)-collider-metastability for these exotic states. An hypothetical detection of DM decays in such a simple setting is therefore expected at LHC, in terms of the presence of $\Sigma_{f}$ metastable tracks or $\Sigma_{f}$ displaced vertices.

\section{Freeze-in/SuperWIMP regime}

As already said, we are interested in the scenario where the DM coupling with ordinary matter is too weak to allow for thermal equilibrium. In this case, we can approximately determine the range of values of $\lambda$ for which DM cannot reach thermal equilibrium (and so be generated by Freeze-in and SuperWIMP mechanisms) by imposing that the ratio of the rate of $\Sigma_{f}$ decay into DM particles to the Hubble expansion rate is lower than one at temperatures of the order of $m_{\Sigma_{f}}$. From this requirement we obtain:

$$
\lambda^{2}<8 \pi \sqrt{g_{*}} 1.66 \frac{m_{\Sigma_{f}}}{M_{P l}} g_{\Sigma}^{-1},
$$

where $g_{\Sigma}$ and $g_{*}$ denote the number of the internal degree of freedom of the FIMP $\Sigma_{f}$ and the number of relativistic degree of freedom of the primordial plasma at the time of DM production, respectively. Specifically, note that the upper bound $\lambda \lesssim 10^{-7}$ can be found for $\Sigma_{f}$ masses within the reach of $\mathrm{LHC}^{4}$.

If we assume a negligible initial DM abundance, DM can be generated from the combination of Freeze-in and SuperWIMP. In accordance with the Freeze-in mechanism, the DM relic density is given by Equation (2.39), which can be now rewritten as:

$$
\Omega_{\psi}^{F I} h^{2}=\frac{1.09 \times 10^{27} g_{\Sigma}}{g_{*}^{3 / 2}} \frac{m_{\psi} \Gamma\left(\Sigma_{f} \rightarrow \psi f\right)}{m_{\Sigma_{f}}^{2}} .
$$

By using Equation (1.86), it is now possible to rewrite the previous formula as:

$$
\Omega_{\psi}^{F I} h^{2}=\frac{1.09 \times 10^{27} g_{\Sigma_{f}}}{g_{*}^{3 / 2}} \frac{\lambda^{2} x}{8 \pi}
$$

\footnotetext{
${ }^{3}$ Note that other possible non-thermal mechanisms (e.g. production via inflation decay or during prereheating/reheating) are here neglected

${ }^{4}$ We assume that the mass scale produced by LHC has the upper bound: $m_{\Sigma_{f}} \lesssim 2 \mathrm{TeV}$.
} 
where $x=m_{\psi} / m_{\Sigma_{f}}$ and, for simplicity, we have again assumed the scalar field to be much heavier than the DM candidate to neglect kinematical suppression factors in the decay rate.

On the basis of the SuperWIMP mechanism, the SuperWIMP DM relic density is instead given by Equation (2.38), which for the studied setup yields the contribution:

$$
\Omega_{\psi}^{S W} h^{2}=x \operatorname{Br}\left(\Sigma_{f} \rightarrow \psi f\right) \Omega_{\Sigma_{f}} h^{2} .
$$

Here $\Omega_{\Sigma_{f}} h^{2}$ stands for the relic density of $\Sigma_{f}$ computed as if it were stable. Let us stress that in this case the DM relic density is proportional to the branching ratio of $\Sigma_{f}$ into DM $\left(\operatorname{Br}\left(\Sigma_{f} \rightarrow \psi f\right)\right)$ since more than one decay channel is now open. As a result, this abundance could in principle be measured at LHC if both the $\Sigma_{f}$ decays are accessible.

These two contributions to the DM relic density are very different from one another. Whereas the Freeze-in contribution indeed depends only on the interactions between DM and the scalar field, mediated by $\lambda$, the SuperWIMP contribution depends also on the gauge interactions determining the relic density of the scalar field, and on both couplings $\lambda$ and $\lambda^{\prime}$ (encoded in the branching ratio). Because of this feature, several possible scenarios may appear depending on the quantum numbers of $\Sigma_{f}$. Furthermore, we expect $\Omega_{\Sigma_{f}}$ to be very low for a charged relic, as consequence of the efficient interactions of the scalar field, thus suppressing the SuperWIMP contribution at low scalar masses.

Since FIMP and SuperWIMP mechanisms take place at quite different cosmological epochs, the DM relic density can be simply evaluated as the sum of their distinct contributions to the total DM relic density:

$$
\begin{aligned}
& \Omega_{\psi} h^{2}=\Omega_{\psi}^{F I} h^{2}+\Omega_{\psi}^{S W} h^{2} \\
& \approx 1.09 \times 10^{27} \frac{g_{\Sigma}}{g_{*}^{3 / 2}} \frac{x \Gamma\left(\Sigma_{f} \rightarrow f D M\right)}{m_{\Sigma}}+x B r\left(\Sigma_{f} \rightarrow f D M\right) \Omega_{\Sigma} h^{2} \\
& \approx x B r\left(\Sigma_{f} \rightarrow f D M\right)\left[0.717 \frac{g_{\Sigma}}{g_{*}^{3 / 2}}\left(\frac{1 \mathrm{~s}}{\tau_{\Sigma}}\right)\left(\frac{1 \mathrm{TeV}}{m_{\Sigma}}\right)+\Omega_{\Sigma} h^{2}\right] .
\end{aligned}
$$

In order to prove the validity of such an approximation, a numerical approach consisting of solving the coupled Boltzmann equations for the DM and the scalar field can be used [8].

Equation (2.49) shows that both production mechanisms are inefficient if the branching fraction of $\Sigma_{f}$ decay into DM becomes too small. On the other hand, a too large DM coupling to $\Sigma_{f}$ can easily cause DM overproduction. By imposing the cosmological value of the DM density for $\psi$, we can fix some definite ranges of the couplings $\lambda, \lambda^{\prime}$ or equivalently $\tau_{\Sigma_{f}}, \operatorname{Br}\left(\Sigma_{f} \rightarrow f \psi\right)$ as a function of the mass scales, namely $m_{\psi}$ and $m_{\Sigma_{f}}$.

In Chapter 4 we investigate the reach of LHC in the detection of the charged field $\Sigma_{f}$ along with which signal can be expected in the parameter regions favored by a successful cosmological DM production and possible DM decay. 


\title{
Chapter 3
}

\section{Long-Lived stop at LHC}

\author{
"I think we are on the verge of a revolution in our understanding of \\ the Universe. And now I'm sure people have said that before, but \\ the LHC is certainly, by far, the biggest jump into the unknown." \\ Brian Cox
}

In this chapter we consider a scenario where a gravitino LSP and DM candidate, as well as a stop NLSP, are the lightest SUSY states. Particularly, we investigate the LHC production and decay of stop NLSPs in such a scenario both within R-parity conserving (RPC) and R-parity violating (RPV) supersymmetry. To obtain a viable cosmological gravitino DM, bounds coming from $\mathrm{BBN}$ and the gravitino abundance are carefully taken into account. We remark that stop NLSP decays have been previously considered from the cosmological perspective in [104-106] and at the LHC as prompt decays in [107-111].

The expected signal from this model consists in long-lived stops at the collider. They can appear either as displaced vertices or as metastable particles. The former are vertices that occur away from the primary vertex (point at which the protons collided), whereas the latter are particle leaving the detector before decaying.

We have three goals. Firstly, we aim at determining the LHC reach for direct stop production, regardless of the decay channel, and comparing the parameter region with the cosmologically viable one. Secondly, we aim at distinguish the R-parity conserving and RPV stop decays, if they occur within CMS, in particular inside Pixel and Tracker. Finally, we aim at suppressing possible background from misidentification or underlying events.

In order to better appreciate our scenario, and so its results, we emphasize below the four motivations which led us to it. Firstly, stop is the supersymmetric scalar particle with the largest left-right mixing. Hence, it can naturally be the lightest colored state and consequently more efficiently produced at the collider. Secondly, stop provides usually the largest correction to the Higgs mass, so its mass cannot be too large so as to retain a light Higgs [112-114]. Thirdly, the LHC constraints on stops are much weaker than those on the gluino and first/second generation squarks. The stop production cross-section is indeed smaller and its decay into top or bottom is more difficult to observe. Finally, a considerable fraction of the long-lived NLSPs might still decay inside the detector even for average decay lengths larger than detector size. 
Lastly, we notice that some scenarios with long-lived particle at the detector have already been studied from both a theoretical and experimental point of view, for instance in [51,115-122]. Particularly, in the last years a lot of attention has been given especially to the case of neutralino [123-128], stau [129-136] or sneutrino [137-141] long-lived NLSP, since those states are more likely to be NLSP in the CMSSM or NUHM models. Recently also the case of Higgsino NLSP was considered in [142].

Equipped with the theoretical and cosmological framework for gravitino LSP and stop NLSP, we begin this chapter by imposing the cosmological constraints from BBN and DM density on our scenario. Later we introduce our analysis for the case of displaced vertices in Pixel and Tracker detectors and for the case of metastable particle (or metastable tracks). In the end, we discuss how to disentangle R-parity conserving stop decay from RPV one and reduce background.

\subsection{Stop NLSP couplings, production and decay channels}

In this section we investigate the interactions of the stop NLSP in SUSY models with gravitino LSP and DM candidate. In order to perform it, we make reference to Section 1.3 of Chapter 1.

The main interactions of the stop NLSP are the RPC QCD couplings, which in general dominate the stop pair production. In fact the RPV couplings considered here are many orders of magnitude smaller than the QCD gauge coupling and too suppressed to give a measurable single-stop production. In the limiting case when the rest of the colored states are too heavy to be produced efficiently, the stop production cross-section is dominated by the direct production via the quark-antiquark annihilation and the gluon fusion channels. Thus the stop mass is the only supersymmetric parameter influencing the production cross-section at tree-level and the dependence on the stop mixing arises only at NLO [143].

In this chapter we simulate the stop pair production at LHC through MADGRAPH 5 [144], which includes only the LO cross-section and therefore neglects any mixing angle dependence of the production. Since the NLO corrections can change the cross-section by a of factor 50$70 \%$ within the mass range investigated here [143], it is important to take such corrections into account in our results. To this end, we multiply our LO cross-section by a constant NLO k-factor of 1.6 and neglect the very weak dependence on $\theta$. In Figure 3.1 we display the obtained NLO cross-section $\sigma$ as a function of $m_{\tilde{t}}$ (green line), along with the minimal value of the production cross-section required to give 10 generated particles at $L=\{300,3000\} \mathrm{fb}^{-1}$ (horizontal red lines). Here the intersections give approximately the maximal mass reaches $\{1800,2200\} \mathrm{GeV}$, respectively obtained at $L=\{300,3000\} \mathrm{fb}^{-1}$. Notice that the power-law formula for the NLO cross-section, exploited to plot Figure 3.1, has been obtained by fitting the product of MadGraph data times the NLO k-factor. It reads:

$$
\begin{aligned}
\sigma\left(m_{\tilde{t}}\right)=1 \mathrm{pb} \exp [ & -0.0048\left(\frac{m_{\tilde{t}}}{1 \mathrm{GeV}}\right)+2.79 \times 10^{6}\left(\frac{m_{\tilde{t}}}{1 \mathrm{GeV}}\right)^{-2}-1.47 \times 10^{9}\left(\frac{m_{\tilde{t}}}{1 \mathrm{GeV}}\right)^{-3}+ \\
& \left.+3.16 \times 10^{11}\left(\frac{m_{\tilde{t}}}{1 \mathrm{GeV}}\right)^{-4}-2.44 \times 10^{13}\left(\frac{m_{\tilde{t}}}{1 \mathrm{GeV}}\right)^{-5}-1.71\right] .
\end{aligned}
$$

We highlight that the number 10 is related to the criterion for discovery used throughout this thesis. We will go back to this point later.

Let us now consider the decay channels of the stop and anti-stop pairs both in the RPC and RPV models. If we start from the R-parity conserving model, the interaction stop-gravitino is described by the R-parity conserving gravitino interaction Lagrangian of Equation (1.77). 


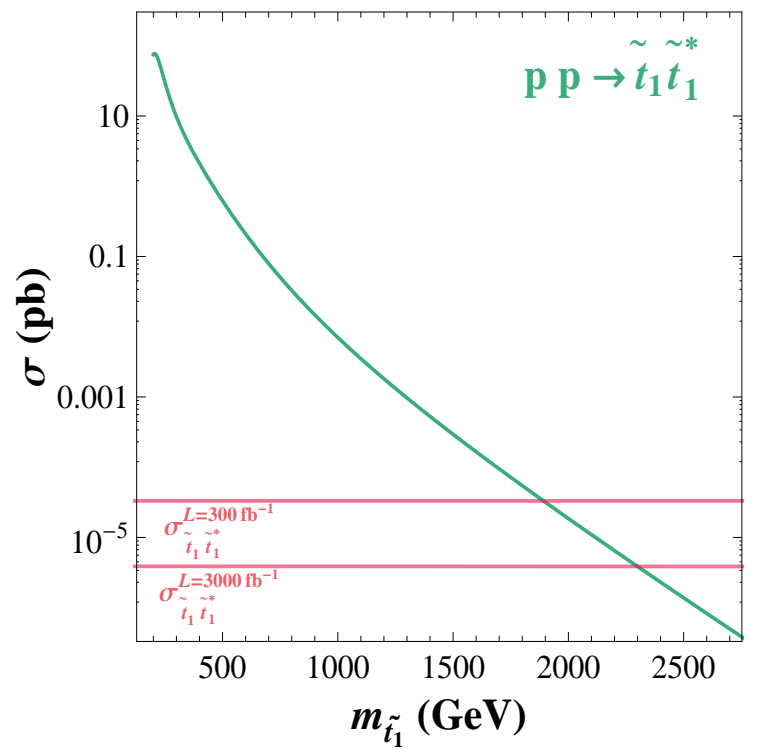

FIGURE 3.1: NLO production cross-section for stop (green line). The minimal value of the cross-section needed to produce 10 stops at $L=300$ and $3000 \mathrm{fb}^{-1}$ (red lines).

Specifically, the term of this Lagrangian we are interested in is:

$$
\mathcal{L}_{3 / 2}=-\frac{1}{M_{P_{r}} \sqrt{2}}\left[\left(D_{\nu} \tilde{t}_{R / L}\right)^{*} \bar{\psi}_{3 / 2}^{\mu} \gamma^{\nu} \gamma_{\mu} P_{R / L} t+\text { h.c. }\right]
$$

where the covariant derivative $D_{\nu}$, the projection operators $P_{L}$ and $P_{R}$ and the reduced Planck mass $M_{P_{r}}$ have already been defined in Section 1.3. Particularly, the reduced Planck mass used in the previous equation has been taken in natural units. If we now express in Equation (3.2) the interaction eigenstates $\tilde{t}_{L}, \tilde{t}_{R}$ by its the mass eigenstates $\tilde{t}_{1}, \tilde{t}_{2}$ (see Equation 1.47 ), we can easily obtain the coupling for the lightest stop $\tilde{t}_{1}$ as:

$$
\mathcal{L}_{3 / 2}=-\frac{1}{M_{P} \sqrt{2}}\left[\left(D_{\nu} \tilde{t}_{1}\right)^{*} \bar{\psi}_{3 / 2}^{\mu} \gamma^{\nu} \gamma_{\mu}\left(-\sin \theta P_{R}+\cos \theta P_{L}\right) t+\text { h.c. }\right] .
$$

By taking the matrix element at the leading order $1 / m_{3 / 2}^{2}$, the decay rate is independent from the mixing angle, which appears only in the interference at order $m_{t} / m_{3 / 2}$, and is given by:

$$
\Gamma_{\tilde{t}_{1}}=\frac{\left(m_{\tilde{t}_{1}}^{2}-m_{3 / 2}^{2}-m_{t}^{2}\right)^{4}}{48 \pi m_{3 / 2}^{2} M_{P}^{2} m_{\tilde{t}_{1}}^{3}}\left[1-\frac{4 m_{3 / 2}^{2} m_{\tilde{t}_{1}}^{2}}{\left(m_{\tilde{t}_{1}}^{2}-m_{3 / 2}^{2}-m_{t}^{2}\right)^{2}}\right]^{3 / 2},
$$

where $m_{t}=173 \mathrm{GeV}$ is the top mass, $m_{\tilde{t}_{1}}$ is the lighter stop mass and $m_{3 / 2}$ the gravitino mass. If we neglect the top mass and the gravitino mass in the phase-space, the stop lifetime reads:

$$
\tau_{\tilde{t}_{1}}=\Gamma_{\tilde{t}_{1}}^{-1} \simeq(18.8 \mathrm{~s})\left(\frac{500 \mathrm{GeV}}{m_{\tilde{t}_{1}}}\right)^{5}\left(\frac{m_{3 / 2}}{1 \mathrm{GeV}}\right)^{2} .
$$

We see that the stop lifetime can cover a very large range of different values. In fact, if we set $m_{\tilde{t}_{1}}=800 \mathrm{GeV}$ and $m_{3 / 2}=\{0.1 \mathrm{GeV}, 100 \mathrm{GeV}\}$ in the previous formula, the two values of the stop lifetime $\tau_{\tilde{t}_{1}} \simeq\left\{0.018 \mathrm{~s}, 1.9 \times 10^{4} \mathrm{~s}\right\}$ can be found, respectively. 
Let us now move onto the bilinear RPV model, given by [35] and summarized in Section 1.2. According to Equation (1.55) and (1.56), the lightest stop can decay via the $h^{\prime}$-type couplings, through its LH component. The relevant term is $-y_{d} \eta_{i j} \epsilon_{k} L_{i}^{\prime} Q_{j} D_{k}^{c}$. The corresponding RPV lagrangian is thus given by:

$$
\mathcal{L}=\sqrt{2} \frac{m_{b}}{v \cos \beta} \zeta_{i} \sin \theta \tilde{t}_{1} \bar{b} P_{L} \ell_{i}+\text { h.c. }
$$

where $v$ is the Higgs vacuum expectation value, the fields $\ell$ and $b$ stand for a lepton and a bottom quark respectively and, finally, $m_{b}$ denotes the bottom mass.

In the rest frame of the decaying stop $\tilde{t}_{1}$, if the antilepton masses are neglected, the total decay rate for a flavour-democratic case $\epsilon_{i}=\epsilon$ reads then:

$$
\Gamma_{\tilde{t}_{1}}=\frac{3 m_{\tilde{t}_{1}}}{8 \pi}\left(\frac{\epsilon \sin \theta m_{b}}{v \cos \beta}\right)^{2}\left(1-\frac{m_{b}^{2}}{m_{\tilde{t}_{1}}^{2}}\right)^{2},
$$

and thus, by using the explicit value for the Higgs vacuum expectation value and the bottom mass $m_{b}=4 \mathrm{GeV}$, the corresponding lifetime $\tau_{\tilde{t}_{1}}$ is:

$$
\tau_{\tilde{t}_{1}}=4.3 \times 10^{-7} \mathrm{~s}\left(\frac{\epsilon \sin \theta / \cos \beta}{10^{-8}}\right)^{-2}\left(\frac{500 \mathrm{GeV}}{m_{\tilde{t}_{1}}}\right)^{-1} .
$$

For the sake of completeness, note that this stop decay is also present in the case of trilinear lepton number RPV, whereas it is absent in the case of baryon number violating RPV. Furthermore, let us point out that we consider here this particular R-parity violating stop decay as particularly promising because it contains leptons in the final state, which can be more easily detected at collider experiments even when they also occur away from the interaction point and are therefore a very favorable signal.

In the case of baryonic violating R-parity violation or even in MFV models like $[145,146]$, the relevant superpotential coupling for the lightest stop is given by the $\lambda^{\prime \prime}$ coupling of Equation (1.49) and the lagrangian reads instead:

$$
\mathcal{L}=\sqrt{2} \lambda_{3 j k}^{\prime \prime} \cos \theta \tilde{t}_{1} \bar{d}_{j} P_{L} d_{k}^{c}+\text { h.c. },
$$

with $\lambda_{3 j k}^{\prime \prime}$ antisymmetric on the last two indices, giving the decay rate into two light-quark jets as

$$
\Gamma_{\tilde{t}_{1}}=\frac{m_{\tilde{t}_{1}}}{8 \pi}\left(\lambda_{321}^{\prime \prime} \cos \theta\right)^{2} .
$$

For small $\lambda^{\prime \prime}$ this decay can also lead to displaced vertices, with two jets originating far away from the stop pair production vertex.

In any of the scenarios discussed here, the stop lifetime is always much longer than the hadronization time. For this reason, we expect stop and anti-stop to hadronize into an Rhadron before they decay, as discussed in $[147,148]$. Such an R-hadron can in principle be both electromagnetically charged or not and even change its charge while it travels in the CMS detector. Nevertheless, since the stop mass is much larger than the QCD confinement scale and the mass of the Standard Model light quarks, we do not expect the fact that stops are bounded to influence substantially their lifetime. In this case, indeed, any phase space suppression due to the final state is negligible and Heavy (s)Quark Effective Theory can be applied. Then order 
$O(1)$ hadronic matrix elements for the inclusive decay rate are obtained ${ }^{1}$. Notice that since we scan the stop lifetime in the following, any deviation of the hadronic matrix elements from one can always be absorbed in a rescaling of the stop lifetime compared to the (possibly observed) R-hadron lifetime.

In view of clarity and transparency of exposition, from now on we adopt the convention that the lighter stop $\tilde{t}_{1}$ is simply called $\tilde{t}$.

\subsection{Cosmology of Stop NLPS and gravitino LSP}

In this section we discuss shortly the cosmological bounds on the scenario with stop NLSP and the gravitino DM and LSP, in order to single out the cosmologically preferred parameter space in both RPC and RPV models. Such a cosmologically viable parameter space will be then compared to the LHC reach for direct stop NLSP production we are going to discuss later in this chapter.

Let us consider first of all the effect of a stop NLSP during BBN. The stop is a colored and EM-charged particle and therefore it can disrupt BBN not only through the energy release in the decay, but also because of the bound state effects [101]. In the first case the light element abundances are more strongly affected by hadro-dissociation and therefore the limits are more stringent for hadronically decaying particles like the stop [99,149]. In the latter case the constraints are independent of the decay channel and just depend on the stop lifetime and density at the time of decay $[101,150]$. For this reason, such constraints can be applied equally to any of the scenarios we have discussed.

The limits on the abundance $Y_{\chi}\left(\tau_{\chi}\right)=n_{\chi} / s$ from bound state effects for a hypothetical long-lived strongly interacting massive particle $\chi$, have been computed by Motohiko Kusakabe et al. in [150]. In particular, by requiring that the primordial light element abundances remain within the observed ranges, they obtained the following constraints depending on the particle lifetime $\tau_{\chi}$ :

- $Y_{\chi}<10^{-18}-10^{-12}$ (for $30 \mathrm{~s}<\tau_{\chi}<200-300 \mathrm{~s}$ ),

- $Y_{\chi}<10^{-18}-10^{-21}$ (for $200-300 \mathrm{~s}<\tau_{\chi}<2 \times 10^{3} \mathrm{~s}$ )

- $Y_{\chi}<10^{-21}-10^{-22.6}$ (for $2 \times 10^{3} \mathrm{~s}<\tau_{\chi} \ll 4 \times 10^{17} \mathrm{~s}$ ) .

In the window $\left(30 \mathrm{~s}<\tau_{\chi}<200-300 \mathrm{~s}\right)$ the most stringent constraint comes from the upper limit on the ${ }^{7} \mathrm{Li}$, while for $\left(200-300 \mathrm{~s}<\tau_{\chi}<2 \times 10^{3} \mathrm{~s}\right)$ the strongest constraint is due to the upper limit on the B abundance. Finally the upper limit on the ${ }^{9} \mathrm{Be}$ abundance determines the bound for longer lifetimes. These constraints are very steep and become quickly dominant over the hadro-dissociation bounds [99,149]. For lifetimes shorter than approximately $30 \mathrm{~s}$ the constraints from bound states disappear and those on hadronic decays are very weak [151] and this corresponds in our two scenarios to a stop mass range:

$$
m_{\tilde{t}} \geq\left\{\begin{array}{cc}
451 \mathrm{GeV}\left(\frac{m_{3 / 2}}{1 \mathrm{GeV}}\right)^{2 / 5} & \mathrm{RPC} \\
1.4 \times 10^{-8} \mathrm{GeV}\left(\frac{\epsilon \sin \theta / \cos \beta}{10^{-8}}\right)^{-2} & \mathrm{RPV}
\end{array} .\right.
$$

\footnotetext{
${ }^{1}$ Indeed also for the B-mesons in the limit of infinite $m_{b}$ the hadronic matrix elements for the inclusive decay rate tend to one [23].
} 


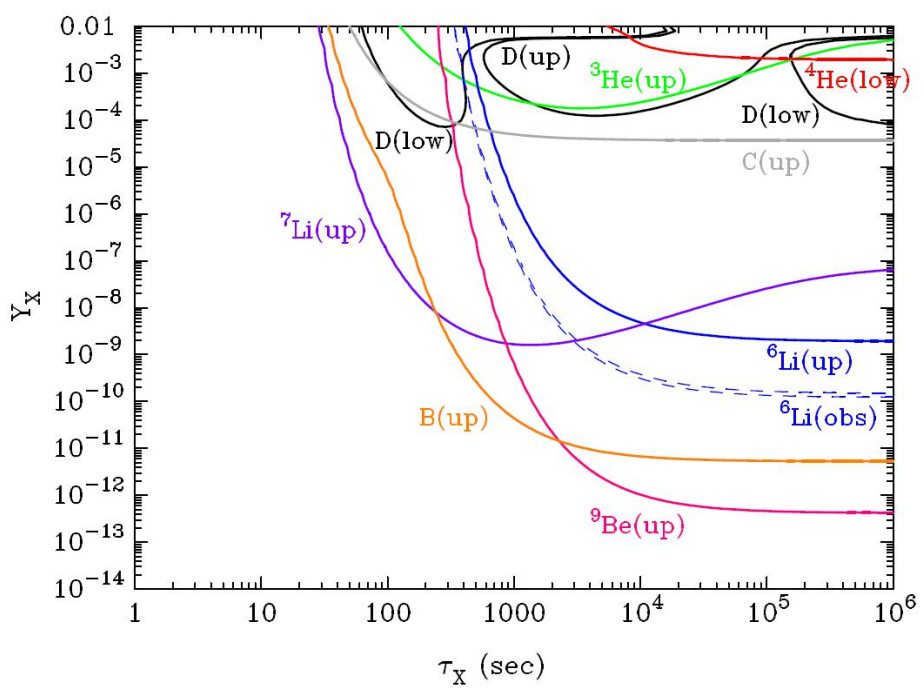

Figure 3.2: Contours for the mass fraction of ${ }^{4} H e, Y_{X}=n_{X} / n_{b}=0.2419$ (red line) and the number ratios of ${ }^{3} \mathrm{He} / \mathrm{H}=3.1 \times 10^{-5}$ (green line), $D / H=3.24 \times 10^{-5}$ and $\mathrm{D} / \mathrm{H}=2.45 \times 10^{-5}$ (black lines), ${ }^{6} \mathrm{Li} / \mathrm{H} \approx 10^{-10}$ (blue solid line) and ${ }^{6} \mathrm{Li} / \mathrm{H}=(7.1 \pm 0.7) \times 10^{-12}$ (blue dashed lines), ${ }^{7} \mathrm{Li} / \mathrm{H}=6.15 \times 10^{-10}$ (purple line), ${ }^{9} \mathrm{Be} / \mathrm{H}=10^{-13}$ (pink line), $\mathrm{B} / \mathrm{H}=10^{-12}$ (orange line) and $C / H=10^{-8}$ (gray line) are shown [150].

We see therefore that BBN does not provide practically any bound on the R-parity violating (RPV) scenario, unless the RPV coupling is very small, below $10^{-12}$. For this reason we will only consider in detail the constraints for the RPC case in the following.

The above limits on the abundance $Y_{\chi}$ can be found in Figure 3.2 if we convert the mass fraction $Y_{X}\left(=n_{X} / n_{b}\right)$ into $Y_{\chi}$ through the formula: $Y_{\chi} \simeq 10^{-10} Y_{X}$. Note that the latter formula can be obtained by using the well-known relation $s=7.04 n_{\gamma}$ along with the approximation $\eta=n_{b} / n_{\gamma} \simeq 5 \times 10^{-10}$ and both $\chi$ and $X$ represent the same hypothetical long-lived particle.

\section{RPC decay of stop NLSP in cosmology}

The relic density of a colored relic like a scalar top and the BBN bounds from hadro-dissociation have been studied in a model independent way in the past by C. Berger et al. in [104]. Below we follow this analysis, but we update the constraints to include also the bound state effects discussed above. In [104] the authors first have considered the simplified case of a single annihilation channel $\tilde{t} \tilde{t}^{*} \rightarrow g g$ in the stop Boltzmann equation. Such a choice is motivated by the fact that such channel just depends on the stop mass and its QCD representation, without dependence on the rest of the supersymmetric spectrum, and, in addition, it is always the dominant channel, contributing at least $50 \%$ of the total annihilation cross-section. It therefore gives the most conservative result since it cannot be suppressed by particular choices of the superparticles spectrum and it provides a reliable upper limit on the stop abundance. In fact, other annihilation channels can only increase the cross section and, therefore, reduce the stop density. In this case the stop abundance is proportional to the stop mass and it reads [104]:

$$
Y_{\tilde{t}}\left(m_{\tilde{t}}\right)=Y_{\tilde{t}}(1 \mathrm{TeV})\left(\frac{m_{\tilde{t}}}{1 \mathrm{TeV}}\right),
$$




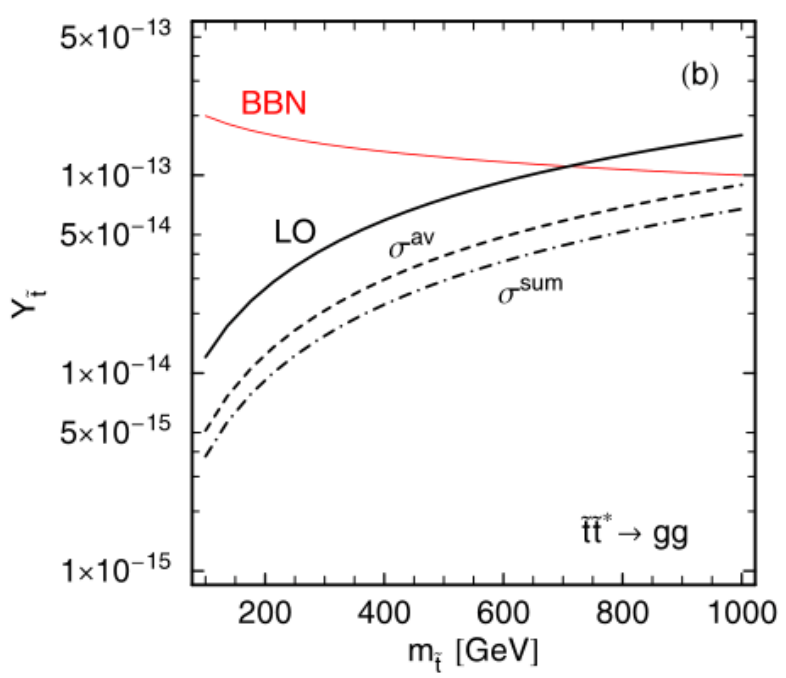

Figure 3.3: The effect of the Sommerfeld enhancement on the yield from $\tilde{t} \tilde{t}^{*} \rightarrow g g$ : the full line shows the tree-level result, the dashed line the result for $\sigma^{a v}$, i.e. applying an averaged Sommerfeld factor, and the dashed-dotted line is for $\sigma^{\text {sum }}$, i.e. applying a summed factor. For more details see [104].

up to logarithmic corrections, since, in general, the mass always appears linearly in the Boltzmann equation for the stop density ${ }^{2}[104]$.

Perturbation theory is commonly used to calculate annihilation and scattering cross sections, with higher-order terms in the perturbative expansion being neglected. Provided that the theory is not strongly coupled, this is generally a good approximation for relativistic particles, however at low velocities and in the presence of a long-range force (classically, when the potential energy due to the long-range force is comparable to the particles kinetic energy), the perturbative approach breaks down. In the non-relativistic limit, the question of how the long-range potential modifies the cross section for short-range interactions can be formulated as a scattering problem in quantum mechanics, with significant modifications to the cross sections occuring when the particle wave functions are no longer well approximated by plane waves (so the Born expansion is not well-behaved). The deformation of the wave functions due to a Coulomb potential was calculated by Sommerfeld in [152], yielding a $\sim 1 / v$ enhancement to the cross section for shortrange interactions (where the long-range behavior due to the potential can be factorized from the relevant short-range behavior).

The computation of such a Sommerfeld enhancement $[153,154]$ for the studied channel $\tilde{t} \tilde{t}^{*} \rightarrow$ $g g$ was also performed in [104], where the authors employed two different prescriptions for the higher orders. Note that the Sommerfeld enhancement increases the cross-section at low velocity and can be obtained by resumming over the exchange of a ladder of gauge bosons between the initial particles. It was found that the averaged Sommerfeld factor reduces the tree-level yield by roughly a factor of 2 , while the summed Sommerfeld one by roughly a factor of 3 , as we can see it in Figure 3.3. We will therefore take the stop abundance from the leading order computation in [104] and vary it by a factor 2-3 to see the effect of both the Sommerfeld enhancement and the additional annihilation channels.

In order to set limits on the RPC model, we compute the stop density as a function of the stop mass from Equation (3.12) and we compare it with the limits in [150]. We determine then

\footnotetext{
${ }^{2}$ Recently it has been discussed in [135] that the stau NLSP abundance is better fitted by a dependence given by $m_{\tilde{\tau}}^{0.9}$ but in the range of masses we are considering such a difference in the exponent has negligible effect.
} 


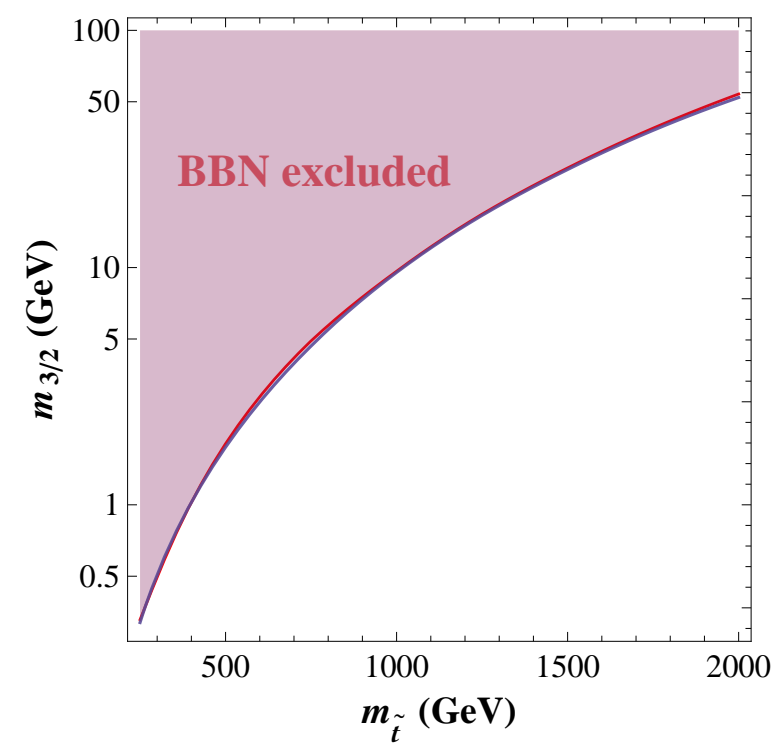

FiguRE 3.4: $B B N$ bounds on the RPC stop NLSP with gravitino LSP scenario. The red and (superimposed) blue regions are excluded by the BBN constraints assuming the $L O$ or the averaged Sommerfeld enhanced stop abundance.

the maximal allowed value of the stop lifetime which corresponds to $Y_{\tilde{t}}=Y_{\chi}^{\text {bound }}$. Through the analytical formula for the stop lifetime, we can then recast the bounds in the plane $m_{\tilde{t}}$ vs $m_{3 / 2}$. We give these results in Fig. 3.4. We see that the constraints for the LO and the Sommerfeld enhanced case overlap and are almost in perfect agreement. This is due to the fact that the bound-state BBN constraints are very steep and do not change appreciably even if the stop density changes by a factor of a few. So the BBN bounds practically do not depend on the details of the stop freeze-out, as long as no strong resonant annihilation is present, and they are quite robust and independent of the masses of the heavier superpartners and therefore of the particular supersymmetric model with stop NLSP ${ }^{3}$.

\section{CDM constraints}

We are assuming in this chapter that gravitinos are Cold Dark Matter and therefore they must have obtained the required abundance in the course of the cosmological evolution. The gravitino production by the decay of the stop NLSP $[98,155]$ is, in most of the parameter space, negligible since either the stop abundance or the gravitino mass are too small. Moreover such contribution is substantial only in the case of the RPC model and it is instead very much suppressed if the stop RPV decay is dominant.

On the other hand, as we have already mentioned in Section 2.6, gravitinos can be generated in substantial numbers by scatterings and decays of supersymmetric particles in equilibrium in the hot plasma. Their abundance is then proportional to the bath reheating temperature $T_{R}$ and can exceed the critical density of the Universe if no restrictions on the reheating temperature is imposed [156-158]. The gravitino abundance is given by Equation (2.41) and in this context

\footnotetext{
${ }^{3}$ We do not consider here the case of coannihilations for the stop, but note that coannihilation with a more weakly-interacting state like the neutralinos/sleptons increases the number density and therefore would correspond to stronger constraints.
} 
it can be rewritten as $[128,156,157]$ :

$$
\Omega_{3 / 2} h^{2} \sim \frac{T_{R}}{10^{9} \mathrm{GeV}}\left(\frac{m_{\tilde{t}}}{300 \mathrm{GeV}}\right)^{2}\left(\frac{m_{3 / 2}}{1 \mathrm{GeV}}\right)^{-1} \sum_{i=1}^{3} \gamma_{i}\left(\frac{M_{i}}{m_{\tilde{t}}}\right)^{2}
$$

where $M_{i}$ are the physical gaugino masses and the coefficients $\gamma_{i}$ account for the Renormalization Group Equation (RGE) effects between the reheating temperature scale and the scale of the physical gaugino masses. We have for those constants the ranges $\gamma_{1}=0.17-0.22, \gamma_{2}=0.54-$ $0.57, \gamma_{3}=0.48-0.52$ from the 1-loop RGE for the gaugino masses and gauge couplings from $T_{R}=10^{7}-10^{9} \mathrm{GeV}[128]^{4}$. For the sake of clarity, it is important to emphasize that we neglect here possible decay of the heavier superpartners in equilibrium, the Feebly Interacting Massive Particle (FIMP) contribution [102], which may even play a dominant role in the case of hierarchical spectra between gauginos and scalar superpartners [159]. Note here the dependence on other supersymmetric masses than the LSP mass, such that the exact abundance becomes a model dependent quantity. Since we are here interested mostly in a constraint on the gravitino and stop masses, we consider as most conservative the case when the gaugino masses are not much heavier that the stop, $M_{i} / m_{\tilde{t}}=(1.1-2)$, in order to minimize the gravitino production.

Although the current best-fit value for DM density comes from the Planck 2015 data [80] and is given in Table 2.2 by:

$$
\Omega_{C D M_{o}} h^{2}=0.1201(38)
$$

during the completion of this work the best-fit value came from the Planck 2013 data [89] and was given by:

$$
\Omega_{C D M_{o}} h^{2}=0.1199(27) .
$$

However, the difference between them is too small in order to modify our results. Note that both values for DM density result slightly larger that the previously obtained combination of the seven-years WMAP data, observations of baryon acoustic oscillations and determinations of the present Hubble parameter in [160],

$$
\Omega_{C D M_{o}} h^{2}=0.1126(36) \text {. }
$$

We impose here that the gravitino energy density in Equation (3.13) is smaller or equal to the the Cold Dark Matter density. From the equality to two measurements $\Omega_{C D M_{o}}=0.1126-0.1199$ we obtain the yellow and brown curves in Figure 3.5. On such lines the gravitino CDM density is fully produced by thermal scatterings, while below the line the gravitinos are overabundant and therefore the parameter space is excluded by the CDM constraint. Specifically, looking at the three couples of CDM density lines of Figure 3.5 obtained for $T_{R}=10^{3}, 10^{4}$ and $10^{7} \mathrm{GeV}$, we can easily note that the position of the these lines depend on the particular $T_{R}$ assumed and the curves move up and down in the value of the gravitino mass exactly by the change in $T_{R}$, due to the the dependence of gravitino density abundance on the ratio $T_{R} / m_{3 / 2}$. Finally, comparing the two plots for different values of $M_{i} / m_{\tilde{t}}$, we can see that for a bigger ratio of physical gaugino masses to stop mass, the gravitino yield is larger and the excluded region, according to the temperature, increases in size.

\footnotetext{
${ }^{4}$ Note that the expression in Equation (3.13) is valid only in the weak coupling limit and breaks down at low reheating temperature $\left(T_{R} \sim 10^{3} \mathrm{GeV}\right)$. Nevertheless, we use it as an indicative curve in Figures 3.5 and 3.6
} 

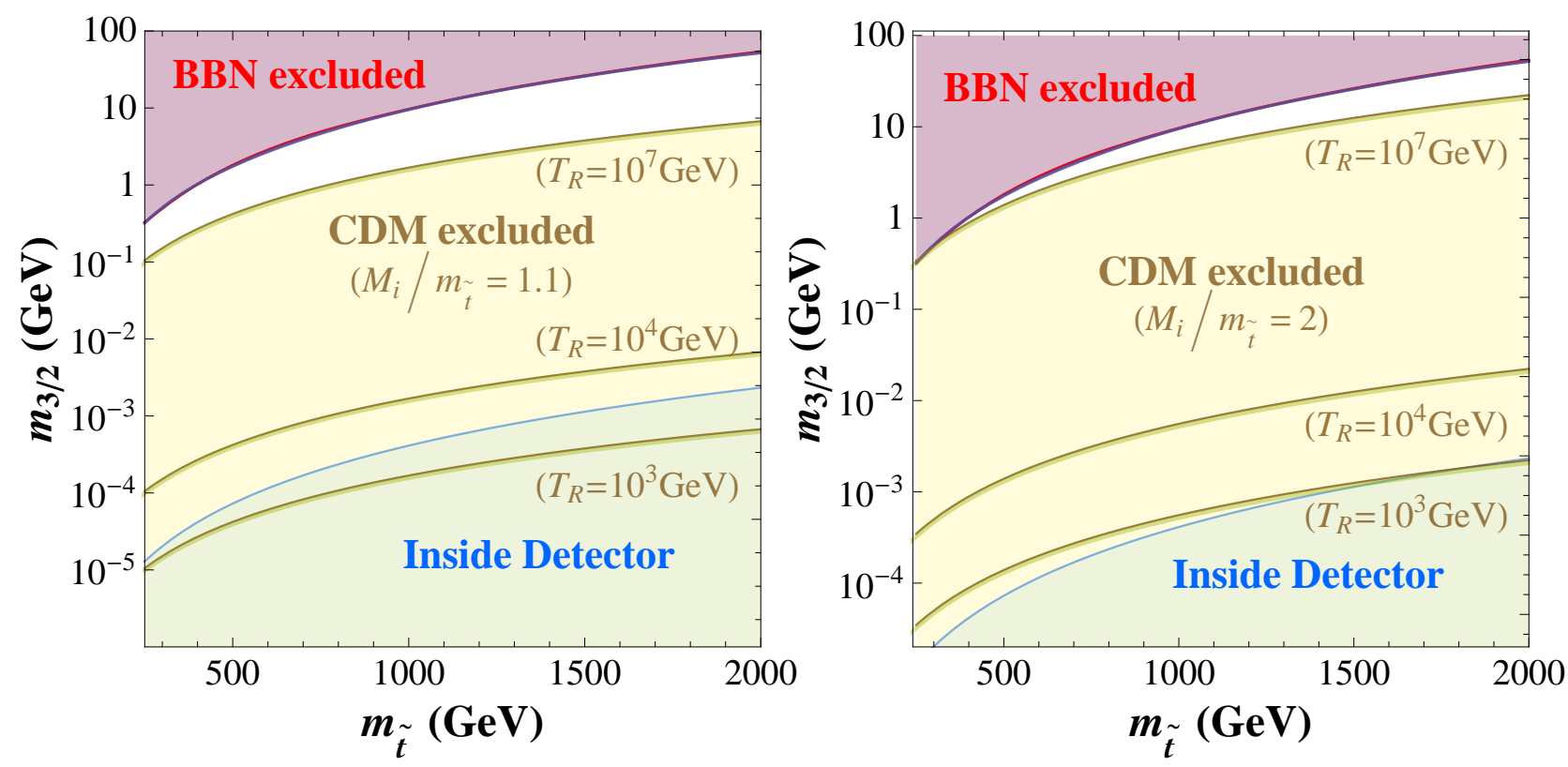

FiguRe 3.5: Plane " $m_{\tilde{t}}$ vs $m_{3 / 2}$ " with the BBN and $C D M$ excluded regions (red and yellow, respectively) and with the Inside detector region (light blue). On the left side (right side) of the figure a CDM excluded region for $T_{R}=10^{3}, 10^{4}, 10^{7} \mathrm{GeV}$ and $M_{i} / m_{\tilde{t}}=1.1(2)$ is drawn. The allowed region is between the BBN and CDM curves and is painted white for the case of $T_{R}=10^{7} \mathrm{GeV}$.

\section{All constraints}

We plot now both the cosmological constraints together in the plane $m_{\tilde{t}} \mathrm{vs} m_{3 / 2}$ in Figure 3.5. For future convenience we also show the region of the parameter space where the RPC stop lifetime is smaller than $10^{-7}$ and therefore the stop decays inside the detector.

Looking at this figure, we see that the allowed region is limited from above by the BBN constraint and from below by the CDM constraint, so that only a narrow allowed strip remains for $T_{R}=10^{7} \mathrm{GeV}$ whereas a bigger allowed strip remains for $T_{R}=10^{4}$ and $10^{3} \mathrm{GeV}$. The breadth of such strip depends both on the supersymmetric spectra and in particular on $M_{i} / m_{\tilde{t}}$ and on the reheat temperature assumed. In particular for reheat temperatures above a few $10^{7}$ $\mathrm{GeV}$ no allowed parameter space remains for stop masses below $2 \mathrm{TeV}$. We note that the region where the RPC decay is sufficiently fast to happen in the detector correspond to a very low reheat temperature of the order of $10^{3}-10^{4} \mathrm{GeV}$, so that in case of high reheating temperature the stops appear as metastable particles at the LHC.

In order to conclude the discussion of the cosmology of a gravitino CDM with stop NLSP, it is worth drawing the reheating temperature corresponding to the right value of Dark Matter density for $m_{3 / 2}=1 \mathrm{GeV}$ and the maximal allowed reheat temperature $T_{R}^{\max }$ as a function of the stop mass in Figure 3.6. Such curves are shown for two different values of the ratio of physical gaugino masses and stop mass in Figure 3.6, i.e. $M_{i} / m_{\tilde{t}}=1.1$ in green (solid line) and $M_{i} / m_{\tilde{t}}=2$ in blue (dot-dashed line). We see that for larger NLSP mass, a smaller reheating temperature is needed to match the observed DM density at fixed gravitino mass, while on the other hand the bound on $T_{R}$ becomes relaxed for larger stop masses as the BBN bounds are weaker. 

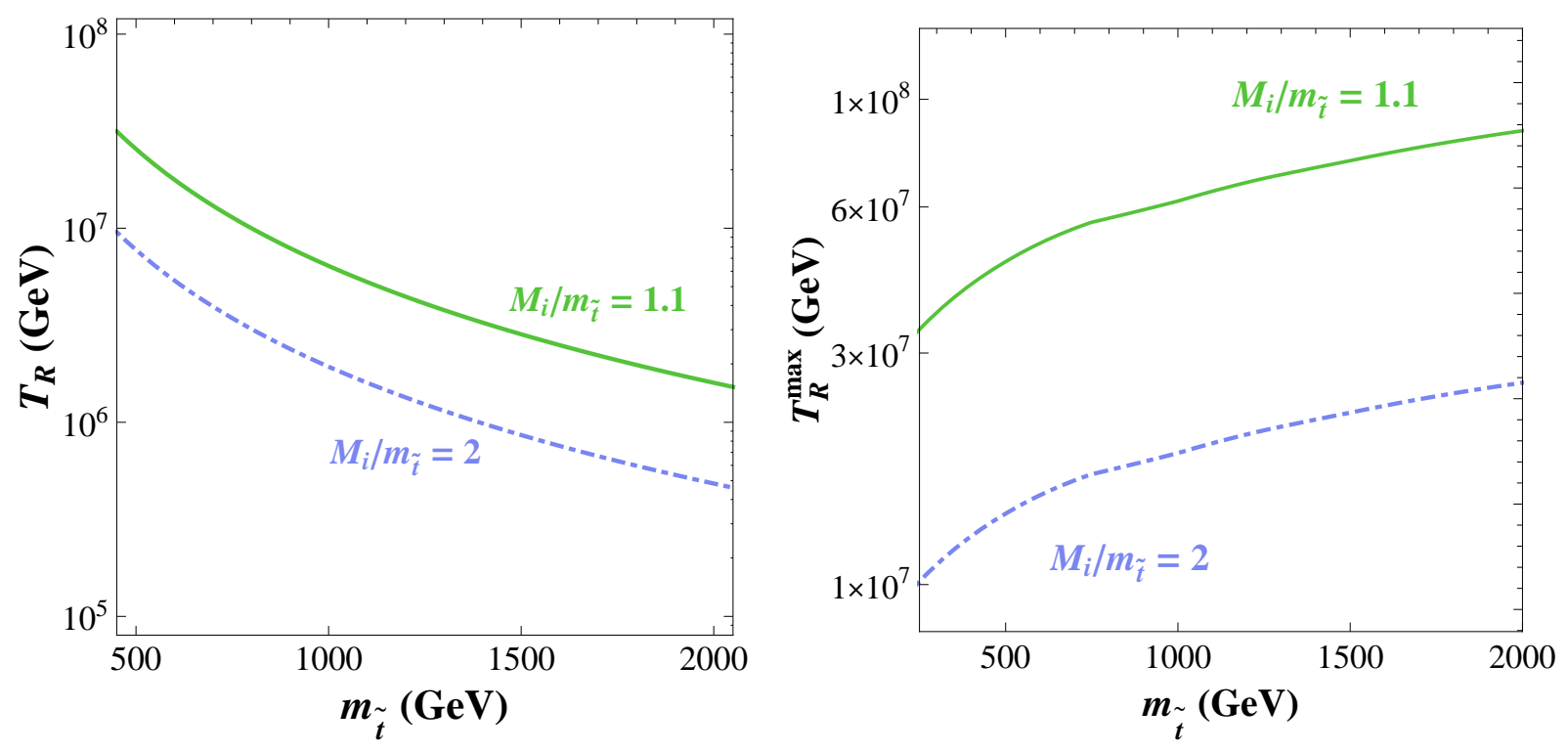

FiguRE 3.6: Plot of the reheating temperature $T_{R}$ as a function of the stop mass $m_{\tilde{t}}$ for the right value of DM density and $m_{3 / 2}=1 \mathrm{GeV}$ on the left panel. Plot of the maximal value of $T_{R}$ as a function of the stop mass $m_{\tilde{t}}$ on the right panel. In both plots the curves for the ratio $M_{i} / m_{\tilde{t}}=1.1$ (green solid line) and $M_{i} / m_{\tilde{t}}=2$ (blue dot-dashed line) are plotted.

\subsection{Decay of stop NLSP at LHC}

Many extensions of the SM include heavy, long-lived, charged particles (HSCPs). These particles can travel distances comparable to the size of modern detectors, where they might be produced. Thus, they might appear to be stable if they have a lifetime bigger that a few nanoseconds. Moreover, the HSCPs can be singly charged $(|Q|=1 e)$, fractionally charged $(|s|<1 e)$, or multiply charged $(|\mathrm{Q}|>1 \mathrm{e})$. Since the particle identification algorithms at hadron collider experiments generally assume signatures appropriate for SM particles, e.g., $v \sim c$ and $Q=0$ or $\pm 1 e$, nowadays the HSCPs might be misidentified or even completely missed without dedicated searches. The LHC experiments have already performed specific analysis, especially for the case of metastable particles [51,117-122].

In this very exciting background the goal of this section is to study two different classes of signal coming from a long-lived stop NLSP, which is produced by the proton-proton collision at LHC, at a center of mass energy of $\sqrt{s}=14 \mathrm{TeV}$ and an integrated luminosities of $L=25 \mathrm{fb}^{-1}$ and $3000 \mathrm{fb}^{-1}$. The first signal is represented by a displaced vertex inside the detector due to the stop decay inside the Pixel or Tracker detector. We will here mostly consider the kinematics and geometry of the CMS detector to estimate the number of decaying events within two adjacent detector parts. We neglect the interactions of the R-hadron with the detector material that could cause the particle to stop in the detector before the decay and the presence of a magnetic field bending the trajectory for charged R-hadron. Such effects could be taken into account only by a full detector simulation, which is beyond the scope of our study. Note in any case that interactions in the detector can only lead to a larger number of events inside the detector and that the magnetic field does not affect neutral R-mesons, which are expected to be around $50 \%$ of the cases [147]. 
The second type of signal is instead the HSCP track of a metastable stop that leaves the detector before decaying. Such a signal is actively searched for by the LHC collaborations $[51,117-122]$.

In both cases we will use MadGraph 5 to compute the LO stop production at LHC and we will correct it with a constant NLO k-factor of 1.6 corresponding to the k-factor given by Prospino [161] for a stop mass of $800 \mathrm{GeV}$. We checked that this factor remains in the range $1.5-1.7$ for stop masses up to $2 \mathrm{TeV}$. Regarding the decay, we will either include it explicitly within the MadGraph 5 analysis with a reference decay rate and then rescale the distances to probe the whole accessible lifetime range or determine from MadGraph 5 just the number of produced stop pairs and use an analytical estimate for the distribution of the decay lengths. As we will see both approaches give similar and consistent results, with the semi-analytical one allowing to explore more easily the parameter space. Before going into detail, we want to highlight here that this analysis is independent from the stop decay channel, as long as the decay gives measurable tracks and a clear displaced vertex. In fact, we will apply our results to the parameter space of both RPC and RPV models that we have already discussed in section 2. We are returning to this crucial point later in this section.

To this day, both the CMS and ATLAS experiments have published searches on this topic for HSCPs produced in proton-proton collisions. Their latest results can be found, respectively, in $[52,162]$. In particular, the CMS HSCP analysis [52] searches for long-lived particles which have a speed significantly less than the speed of light, or a charge $Q$ not equal to $\pm e$, or both. The center of mass energy is set at $\sqrt{s}=8 \mathrm{TeV}$ and an integrated luminosity of $L=18.8 \mathrm{fb}^{-1}$ has been chosen. This analysis relies on using the time-of-flight (TOF) of the particles to the muon system and/or the rate of energy loss $d E / d x$ in the tracker for identification of HSCPs. The five separate channels of investigation are tracker+TOF, tracker-only, muon-only, fractionally-charged and multiply-charged. On the other hand, the ATLAS collaboration [162] searches for HSCPs in various supersymmetric models at a center of mass energy of $\sqrt{s}=8$ $\mathrm{TeV}$ and an integrated luminosity of $L=19.1 \mathrm{fb}^{-1}$. These searches are based almost entirely on the characteristics of the HSCP itself, but are further optimised for the different experimental signatures of sleptons, charginos and R-hadrons.

\section{Numerical analysis}

The numerical analysis in this paper is realized by means of the open source software MadGraph 5 which can generate matrix elements at tree-level, given a lagrangian based model, for the simulation of parton-level events for decay and collision processes at high energy colliders [144]. In order to study the stop production at the LHC experiment we choose the MSSM model from the MadGraph 5 library of models which is built upon that of the package FeynRules ${ }^{5}[163,164]$. Furthermore, we set the centre of mass energy is $\sqrt{s}=14 \mathrm{TeV}$ for all our simulations.

We run MadGraph 5 for several stop masses but for only one reference value of the stop decay rate that we take to be $\Gamma_{\tilde{t}}=2.02159 \times 10^{-10} \mathrm{GeV}$. We generate 10,000 events per run. The kinematics of all particles in the process for every event is recorded and used to assign numerically via a separate Python routine to each stop/antistop particles its own decay length $\ell_{\tilde{t}}$. Such a routine, shown in Appendix A, assumes that the particles propagate undisturbed

\footnotetext{
${ }^{5}$ FeynRules is a Mathematica-based package which addresses the implementation of particle physics models, which are given in the form of a list of fields, parameters and a Lagrangian, into high-energy physics tools. It calculates the underlying Feynman rules and outputs them to a form appropriate for various programs such as CalcHEP, FeynArts, MadGraph, Sherpa and Whizard.
} 


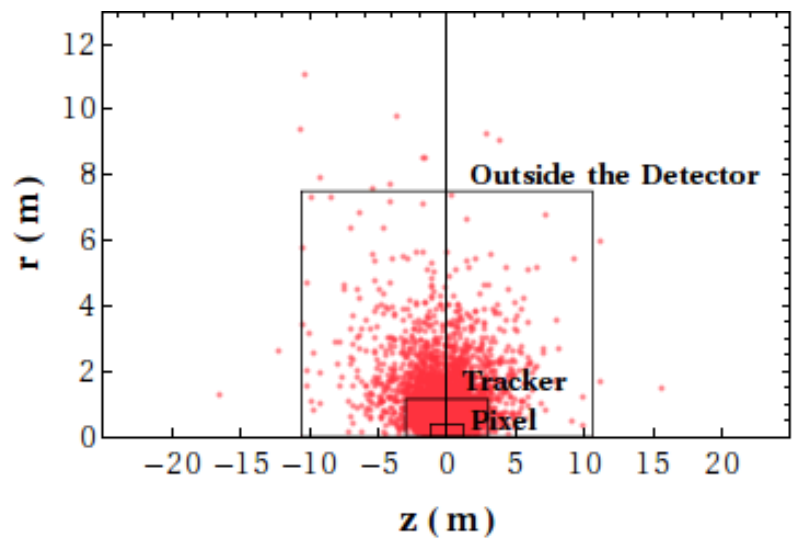

FiguRe 3.7: Distribution of 10,000 stop displaced vertices in the detector plane $(r, z)$ for $m_{\tilde{t}}=800 \mathrm{GeV}$ and $\Gamma=2.02159 \times 10^{-16} \mathrm{GeV}$ along with the size of Pixel, Tracker and the whole CMS detector.

from the interaction point and decay randomly according to an exponential distribution. From the decay length and the stop or antistop momentum direction we can draw the distribution of all decay vertices in the detector plane $(r, z)$ by using the software Mathematica. In appendix B we display the mathematica code we used to analyse the python-output so as to perform our numerical analysis. Since any change in the stop decay rate can be compensated by a corresponding change in the distance that the stop travels, we can circumvent the problem of launching MadGraph 5 for all the decay rates by simply rescaling the dimensions of all parts of the detector consistently. This point will be better clarified in the next subsection when we give the explicit formulas for the semi-analytic analysis. By using this expedient, the spatial distribution of the stop decay vertices for a stop mass of $m_{\tilde{t}}=800 \mathrm{GeV}$ and any stop decay rate can be obtained. We show the results for $\Gamma_{\tilde{t}}=2.02159 \times 10^{-16} \mathrm{GeV}$ in Figure 3.7, where the decay vertices are shown by red dots on top of a schematic view of the CMS detector.

Now equipped with the stop decay vertices, we can count how many stops or antistops decay within the Pixel or Tracker in the CMS detector as a function of the stop lifetime at the integrated luminosity $L=25 \mathrm{fb}^{-1}$ and the maximum expected one $L=3000 \mathrm{fb}^{-1}$. We repeat the same procedure for several stop masses and obtain so an estimate of the LHC reach in the plane of the stop mass versus its lifetime with just two very simple working hypotheses. Firstly we neglect both the backgrounds of the SM and other SUSY particles than the lightest stop, which are respectively expected to have much shorter decay lengths and assumed to be too heavy to be produced at LHC. Secondly we set the detector efficiencies to $100 \%$ and declare 10 decays inside one CMS detector part sufficient for the discovery of a displaced vertex and 10 decays outside for the discovery of a metastable stop. We require 10 decays instead of just 2 or 3 in order to reduce the numerical fluctuations and obtain a more stable numerical result. The analytical estimates in the next section will allow to draw conclusions also for a different number of decays.

We show our results in Figure 3.8, where the data-points corresponding to 10 decays inside Pixel (blue dots), Tracker (red dots) and outside the detector (green dots) are given in the stop mass-lifetime plane at both integrated luminosities, with $L=25 \mathrm{fb}^{-1}$ (left panel) and $L=3000 \mathrm{fb}^{-1}$ (right panel). Here, as in the next plots, the data-points corresponding to 10 tracks leaving the detector are always labeled by "Out". Comparing the Pixel and Tracker reach, we are not surprised to see that they are pretty close to each other since their sizes are similar. 

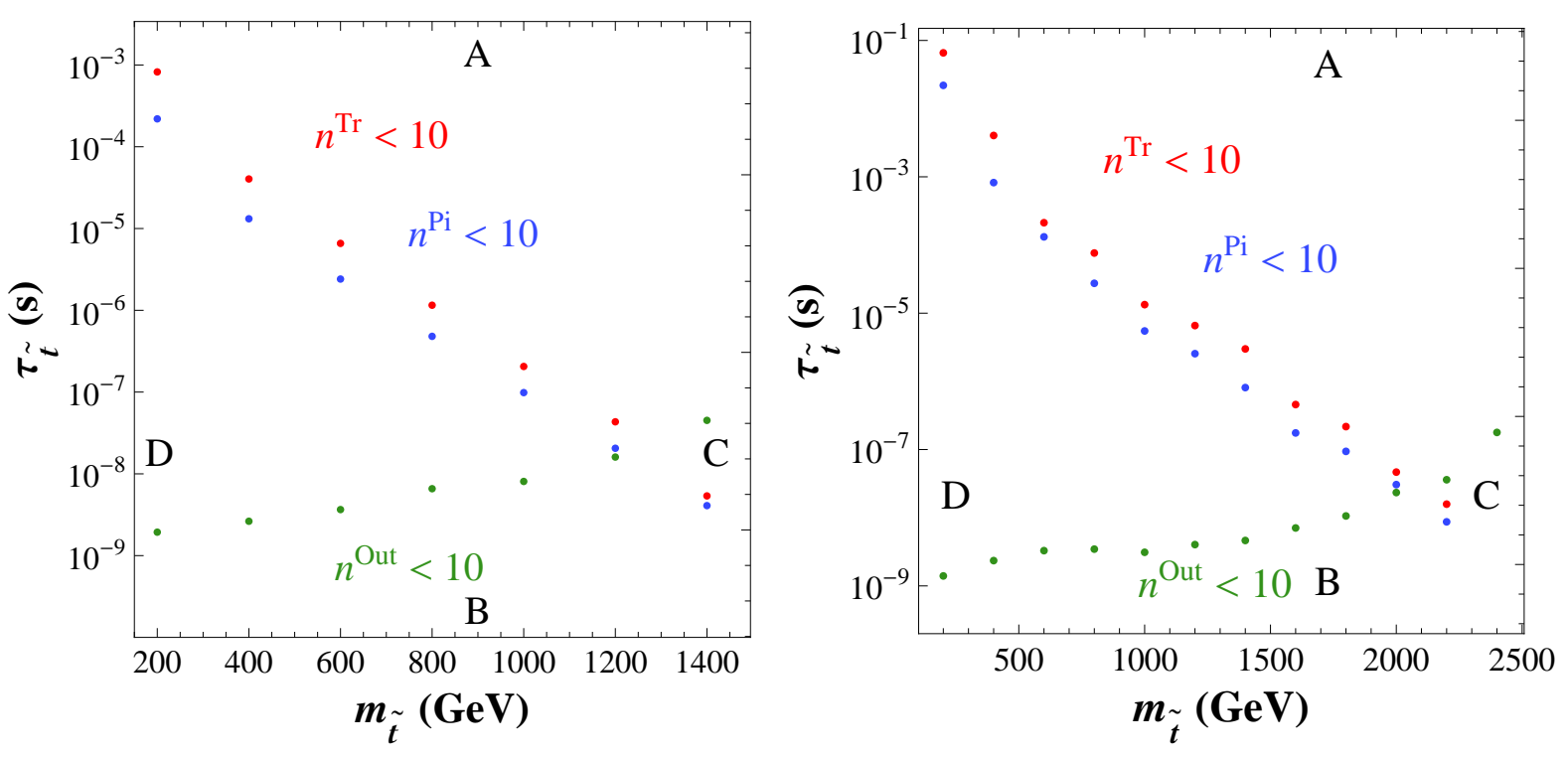

Figure 3.8: $N L O$ LHC reach in the stop lifetime-stop mass plane at $L=25 \mathrm{fb}^{-1}$ in the left panel and $L=3000 \mathrm{fb}^{-1}$ in the right panel. In both panels, the Pixel reach is given by blue points while the Tracker reach by red points. At last, the reach of metastable particles is denoted by green points.

The tracker detector is larger than the pixel one and this gives a slightly better reach, on the other hand we expect that the pixel detector would offer a better precision and efficiency in the measurement of a displaced vertex, so that this may overcome the geometrical advantage in a full detector simulation. It is interesting that the stop kinematical distribution is such that a similar number of events is often obtained in the two detector parts, allowing on one side for a cross-check and on the other to better disentangle a long-lived stop from any SM background like B-mesons decaying mostly in the pixel detector.

Observing Figure 3.8, and interpolating between the points for Pixel/Tracker and outside we can divide the parameter space in four different regions, which we have labeled A, B, C and D. In the three regions $\mathrm{A}, \mathrm{B}$ and $\mathrm{D}$ at least one signal is seen for any stop lifetime. In particular in the region D both types of signal, displaced vertices and metastable tracks, are accessible, allowing to cross-check the measurement of the stop lifetime. Only in region $\mathrm{C}$ no signal could be measured at the LHC. Of course such a region may be reduced by combining the two types of signals to have 10 events in total or by loosening the requirement to fewer events. We see clearly then that the searches for displaced vertices and escaping particles are complementary: the first covers the lower plane corresponding to short lifetimes, i.e. regions D and B, while the latter is mostly sensitive to the long lifetimes in the regions $\mathrm{D}$ and $\mathrm{A}$. Combining the two searches it is possible to cover the parameter space for any lifetime up to a maximal mass where the production cross-section starts to become too small to produce a sufficient number of stops. If the LHC does not observe any signal as displaced vertices and metastable tracks, we can therefore obtain a lifetime-independent lower limit on the stop mass at around $m_{\tilde{t}}=1300 \mathrm{GeV}$ for the integrated luminosity $L=25 \mathrm{fb}^{-1}$ and at around $m_{\tilde{t}} \simeq 2100 \mathrm{GeV}$ for the integrated luminosity $L \simeq 3000 \mathrm{fb}^{-1}$. 


\section{Semi-analytic approximate analysis}

The semi-analytic analysis is realized via analytical estimates for the distribution of decay lengths of the long-lived stop particles instead than via reconstruction of the position of each decay vertex. Even in this case we use MadGraph 5 to compute the production cross section $\sigma$ and, therefore, the number of generated stop particles $N_{0}$ at LHC, from the product of the cross-section times the integrated luminosity $(\sigma L)$. We complement the previous analysis with this semi-analytic approach in order to have a better control of the physical parameter space, faster results and, at the same time, a useful check of the results of MadGraph 5.

The semi-analytic analysis is based on the well-known exponential decay formula for a particle travelling in a straight line, giving the probability $P(d)$ that a particle decays at $d$. It reads:

$$
P(d)=\frac{\Gamma}{\beta \gamma c} \exp \left\{-\frac{\Gamma}{\beta \gamma c} d\right\}
$$

where $\Gamma$ is the decay rate in the centre of mass frame, $c$ the speed of light and $\beta \gamma$ is the relativistic $\beta \gamma$ factor, which is defined in terms of the energy $E$ and the three-momentum $\vec{p}$ of the decaying particle as $\beta \gamma=(|\vec{p}| / E) / \sqrt{1-(|\vec{p}| / E)^{2}}$. The factor in front of the exponential in Equation (3.17) is determined by the proper normalization of the probability $P(d)$, i.e. the condition

$$
\int_{0}^{+\infty} P(d) d d=1 .
$$

By using this exponential decay formula, the corresponding formula for the probability as function of a dimensionless coordinate $y$ can be very easily obtained. It reads:

$$
P(y)=\frac{1}{\beta \gamma} \exp \left\{-\frac{1}{\beta \gamma} y\right\}
$$

where $y=d \Gamma / c$ and the normalization was obtained as for $P(d)$. Here, we can explicitly see that any change in the decay rate $\Gamma$ can be always compensated by an appropriate change in the distance $d$ that the particle travels. Therefore, from the analytical expression of $P(d)$ we can directly justify the rescaling procedure used for the MadGraph events, that allowed us to cover the whole range of decay rates from a single run.

Both the formula for $P(d)$ and $P(y)$ can be generalized to describe an exponential decay of a sample of particles, by integrating over the particles' distribution in momentum and therefore in $\beta \gamma$. With the proper $\beta \gamma$ distribution such an integrated probability should give back exactly the decay length's distribution obtained by the numerical procedure used beforehand. To have a simpler and analytical expression, we will instead assume a single effective "average" value of $\beta \gamma$, defined as $\widetilde{\beta \gamma}$, for all produced particles and estimate the decay length distribution just by multiplying such average single particle probability by the initial number of particles $N_{0}$. In this way, an estimate for the number of decaying particles of the sample as a function of $d$ and 


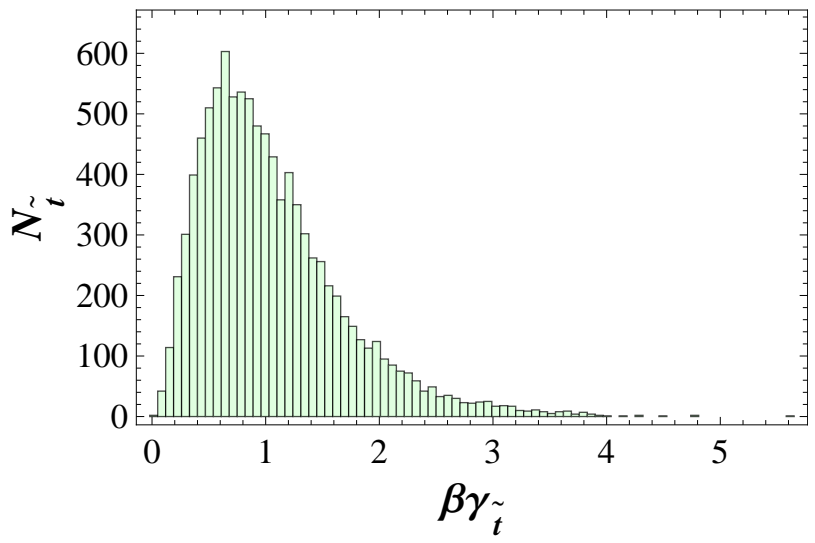

Figure 3.9: MadGraph distribution of the factor $\beta \gamma$ for $m_{\tilde{t}}=800 \mathrm{GeV}$.

$y$, called $N(d)$ and $N(y)$, are achieved. They are:

$$
\begin{aligned}
& N(d)=N_{0} \frac{\Gamma}{\widetilde{\beta \gamma c}} \exp \left\{-\frac{\Gamma}{\widetilde{\beta \gamma} c} d\right\}, \\
& N(y)=N_{0} \frac{1}{\widetilde{\beta \gamma}} \exp \left\{-\frac{1}{\widetilde{\beta \gamma}} y\right\},
\end{aligned}
$$

where the coordinate $d=\sqrt{r^{2}+z^{2}}$ can be taken as a function of the coordinates in the CMS detector. We neglect here in first approximation the bending of the trajectory by the magnetic field, which affects only the case of stop hadronization into a charged hadron, or the energy loss due to the interaction with the detector material, possibly negligible for a stop decay within the inner parts of the detector.

To obtain the optimal value of $\widetilde{\beta \gamma}$ in Equation (3.19), we compute the stop $\beta \gamma$ distribution through MadGraph and try to estimate such quantity from the shape of this distribution. The $\beta \gamma$ distribution is given in Figure 3.9 for a stop mass of $800 \mathrm{GeV}$. We see that even for relatively small masses, the stop and antistop are mostly produced as non-relativistic, with a peak in the $\beta \gamma$ distribution clearly below one. We consider the analytical decay distance distribution with different effective $\widetilde{\beta \gamma}$, i.e. taking the value at the maximum $\beta \gamma^{\max }=0.66$ or $(1 / \beta \gamma)^{\max }=0.8026$ or the average values $\langle\beta \gamma\rangle=0.9207$ or even $\langle\langle 1 / \beta \gamma\rangle\rangle=1.24595$, and we compare those curves with the distribution obtained from the MadGraph run, to see how well these different values of $\widetilde{\beta \gamma}$ work. The value of $\widetilde{\beta \gamma}=\beta \gamma^{\max }=0.66$ gives the best fit of the numerical distribution in the distance range of the pixel and tracker detector. We repeat the same estimate also for larger stop masses and finally we determine a common $\widetilde{\beta \gamma}$ by arithmetic mean of the set of all the $\beta \gamma^{\text {max }}$-factors for all relevant masses. The overall effective $\widetilde{\beta} \gamma=0.66$ turns out to coincide with the value for $m_{\tilde{t}}=800 \mathrm{GeV}$ and we use such value in the analytical Equation (3.19). This $\widetilde{\beta \gamma}$, optimized for detector distances, actually underestimates the decay length's distribution at large distances, but it still gives a conservative estimate for the number of metastable particles decaying outside the detector.

Let us now compute the number of decay vertices in the pixel/tracker or outside directly from the analytical formula of $N(d)$. Assuming just a spherical geometry for the detector parts, we can integrate the expression with respect to $d$ from $r_{i}$ to $r_{f}$, which respectively stand for the initial and radial distance from the IP to the part of the interest of the detector, and from $r_{0}$ to $+\infty$, which instead denote the region outside the detector. In doing so, we have an analytical 
approximate expression for both number of stop decays that occur inside the detector parts and outside the detector. They are given by the equations:

$$
\begin{aligned}
& N_{r_{i} \leqslant d \leqslant r_{f}}=N_{0}\left(\exp \left\{-\frac{\Gamma}{\widetilde{\beta \gamma}} r_{i}\right\}-\exp \left\{-\frac{\Gamma}{\widetilde{\beta \gamma}} r_{f}\right\}\right), \\
& N_{d \geqslant r_{f}}=N_{0} \exp \left\{-\frac{\Gamma}{\widetilde{\beta \gamma}} r_{f}\right\} .
\end{aligned}
$$

Since the number of particles generated by proton-proton collisions is given by the product of cross-section times luminosity, $N_{0}=\sigma L$, and the power-law formula for $\sigma_{(N L O)}\left(m_{\tilde{t}}\right)$ is given by Equation 3.1, we can solve Equation (3.21) and (3.22) for the stop lifetime as a function of the stop mass imposing $N_{r_{i} \leqslant d \leqslant r_{f}}=10$ and $N_{d \geqslant r_{f}}=10$, for the values of $r_{i}, r_{f}, r_{0}$ corresponding to the pixel and tracker in the CMS detector and the whole detector. In this way we can obtain a simple estimate of the LHC reach in the plane stop lifetime versus stop mass that we can apply at different luminosities and also different values of displaced vertices or metastable tracks $N$. We emphasize that the first equation is a transcendental equation and can be only solved numerically, whereas the second can be simply solved analytically. In Figure 3.1 we have plotted the cross-section given by Equation (3.1).

These results are plotted in Figure 3.10, where the dashed blue line, the dot-dashed red line and the green solid line denote, respectively, the Pixel, Tracker and Outside reaches. Observing Figure 3.10, we can note that the crossing analytical curves identify again in the plane the regions, labelled $\mathrm{A}, \mathrm{B}, \mathrm{C}$ and $\mathrm{D}$, which we have discussed earlier. To show the dependence on the requested number of vertices or metastable tracks, we give in Figure 3.11 the LHC reach for $N=\{1,10,100\}$ again for both luminosities $L=25 \mathrm{fb}^{-1}$ (left) and $L=3000 \mathrm{fb}^{-1}$ (right). It is clear here that the change in $N$ affects strongly the reach of the metastable track search only at large masses, since for long lifetimes those events practically coincide to the total number of produced stops and antistops, which decreases very fast as a function of the stop mass. For displaced vertices, the change in $N$ just shifts the curves to a larger/smaller stop lifetime.

Instead in Figure 3.12 we give additional plots of the approximate reach for different LHC luminosities: in the left panel we plot the LHC reach for $L=100 \mathrm{fb}^{-1}$ whereas in the right one the LHC reach for $L=300 \mathrm{fb}^{-1}$. We see from the four different plots in Figures 3.10 and 3.12 that the LHC has the chance to cover the whole parameter space in lifetime up to stop masses of order $1300,1500,1700,2100 \mathrm{GeV}$ for a luminosity of $25,100,300,3000 \mathrm{fb}^{-1}$ respectively.

\section{Comparison and discussion}

To compare directly the numerical and approximate LHC reach in the stop lifetime-stop mass plane, we plot all the curves together in Figure 3.13.

Looking at this figure, it is clear the good agreement between the MadGraph data and the approximate curves at both integrated luminosities: $L=25 \mathrm{fb}^{-1}$, showed in the left panel, and $L=3000 \mathrm{fb}^{-1}$, showed in the right panel. The analytical curves can be easily extended to consider $\pm 1 \sigma$ statistical error bars in the Poisson distribution, corresponding to 8 and 12 events respectively. We see that these curves give a very good description of the numerical data-points as they are mostly included in the $\pm 1 \sigma$ band. Only at low masses, where the statistics is large and the band becomes narrow, the analytical estimate deviates slightly from the numerical results. This is surely partially due to the spherical shape of the detector assumed in the approximated expression, which causes a substantial error in the region with a large number 

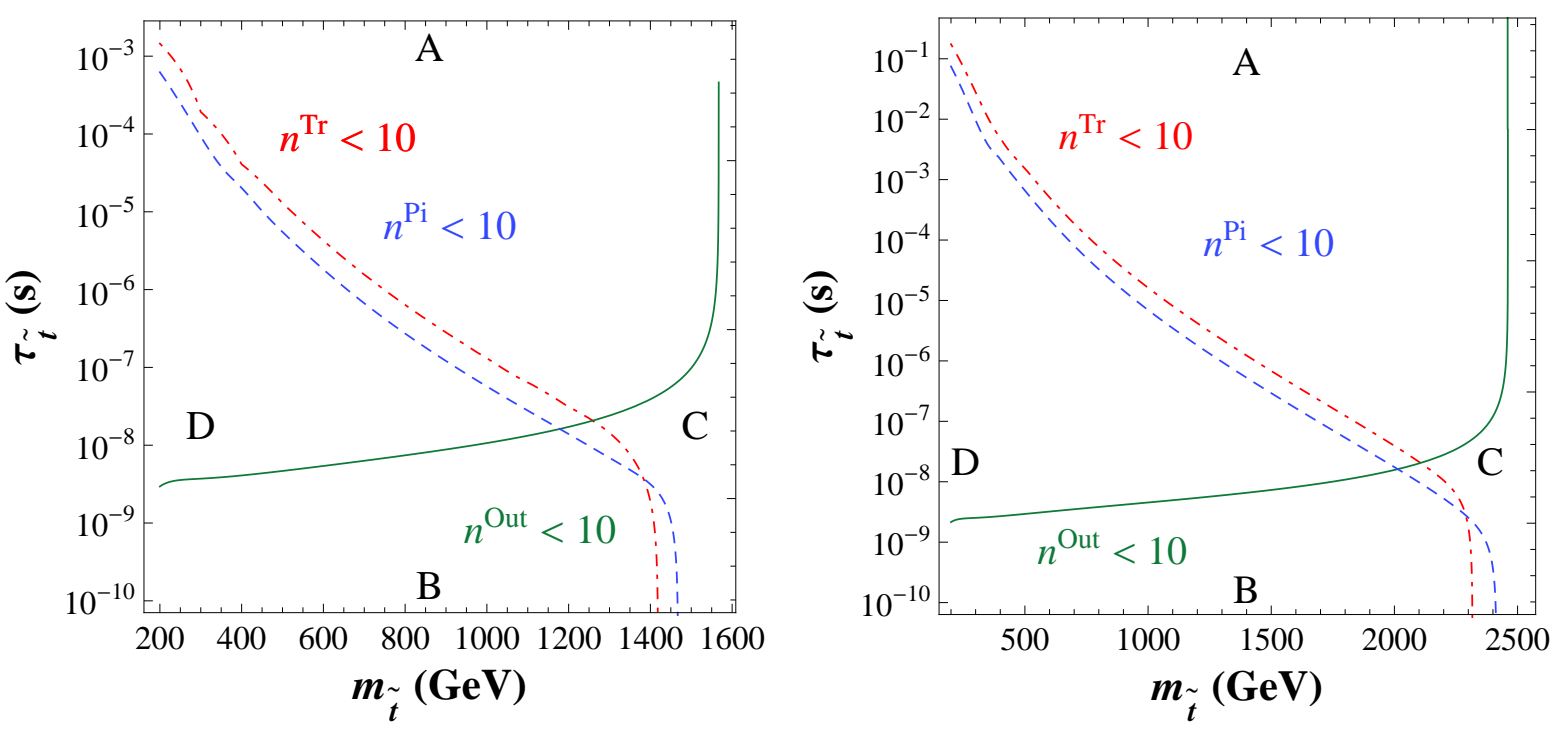

Figure 3.10: Semi-analytical LHC reach in the stop lifetime-stop mass plane at $L=25 \mathrm{fb}^{-1}$ in the left panel and $L=3000 \mathrm{fb}^{-1}$ in the right panel. In both panels, the Pixel reach is denoted by a blue dashed line while the Tracker reach by a red dot-dashed line. The reach of metastable particles is, instead, denoted by a green solid line.

of events. In fact the data-points computed in the numerical analysis with the exact detector shape contain more signal events and give a wider reach in the low mass region.

The current CMS excluded region for metastable particles (MP) [52], obtained at a centre of mass energy of $\sqrt{s}=8 \mathrm{TeV}$ and an integrated luminosity $L=18.8 \mathrm{fb}^{-1}$, given by our analytical curve for zero decays outside the detector, is also shown as a yellow region in the upper left corner of each panel. We see that for long lifetimes the curve excludes stop masses below 800 $\mathrm{GeV}$ and this coincide with the results obtained in [24]. Such bounds weakens for lifetimes below $10^{-7} \mathrm{~s}$. Here it is worth highlighting that the current excluded region still leaves a considerable allowed region to be investigated at LHC in the future.

Finally, since processes involving colored particles are strongly affected by QCD NLO corrections that tend to increase the cross-section, it is also interesting to investigate quantitatively the impact of these corrections. In Figure 3.14 we redraw the same plots without taking into account the NLO corrections, i.e. the NLO k-factor of 1.6, and we observe that such corrections increase the yield substantially and extend the reach to larger stop masses. Indeed the NLO LHC reach in stop mass moves up by approximately $200 \mathrm{GeV}$, i.e. from $1200 \mathrm{GeV}$ to $1400 \mathrm{GeV}$ for $L=25 \mathrm{fb}^{-1}$ and from $2000 \mathrm{GeV}$ to $2200 \mathrm{GeV}$ for $L=3000 \mathrm{fb}^{-1}$.

\subsection{LHC reach for the RPC/RPV stop decay}

As we have already mentioned beforehand, the discussion so far has been centered on the presence of a displaced vertex or metastable tracks and is so independent from the particular stop decay channel, as long as it contains sufficiently many charged tracks to make the secondary vertex visible. Therefore, our analysis is valid for both the RPC and the RPV stop decays we have discussed earlier. We can therefore interpret our results in terms of the two different model parameters. 

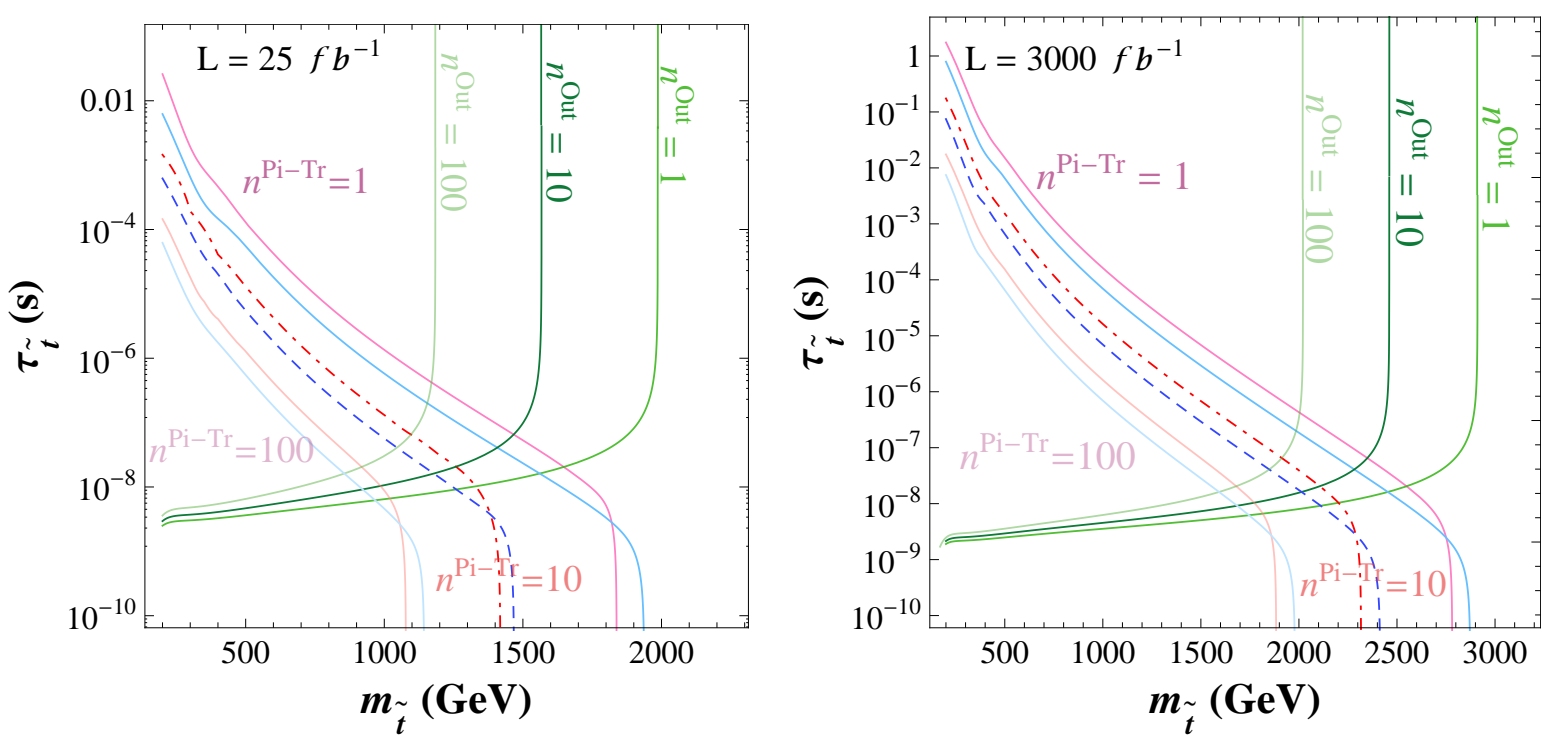

Figure 3.11: Semi-analytical LHC reach in the stop lifetime-stop mass plane at both luminosities $L=25 \mathrm{fb}^{-1}$ (left panel) and $L=3000 \mathrm{fb}^{-1}$ (right panel) and for $N=\{1,10,100\}$.
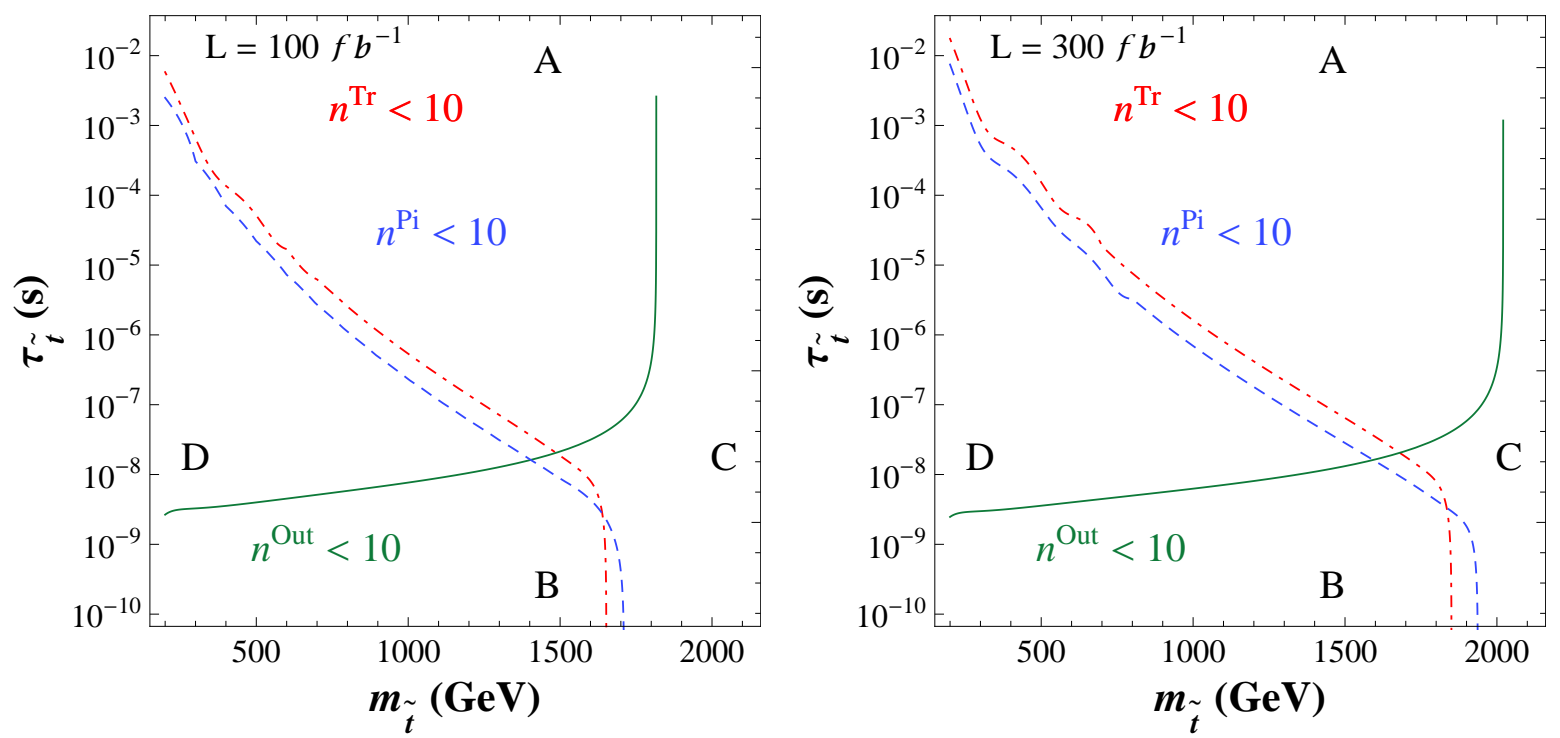

FiguRE 3.12: Semi-analytical LHC reach in the stop lifetime-stop mass plane at the luminosity $L=100 \mathrm{fb}^{-1}$ (left panel) and $L=300 \mathrm{fb}^{-1}$ (right panel).

\section{LHC reach for the RPC stop decay}

For the RPC stop decay into gravitino and top, the LHC reach can be easily reformulated in the plane $m_{\tilde{t}}$ vs $m_{3 / 2}$ by using the analytical formula for the RPC stop lifetime, Equation (3.5). In Figure 3.15 we display these curves at NLO for Pixel, Tracker and the part outside the detector by means of a dashed green line, a dot-dashed blue line and a solid pink line respectively. Here we also plot the BBN excluded region (red region), the CDM excluded region (yellow region) and the current excluded region for metastable particles (yellow region). The CDM excluded 

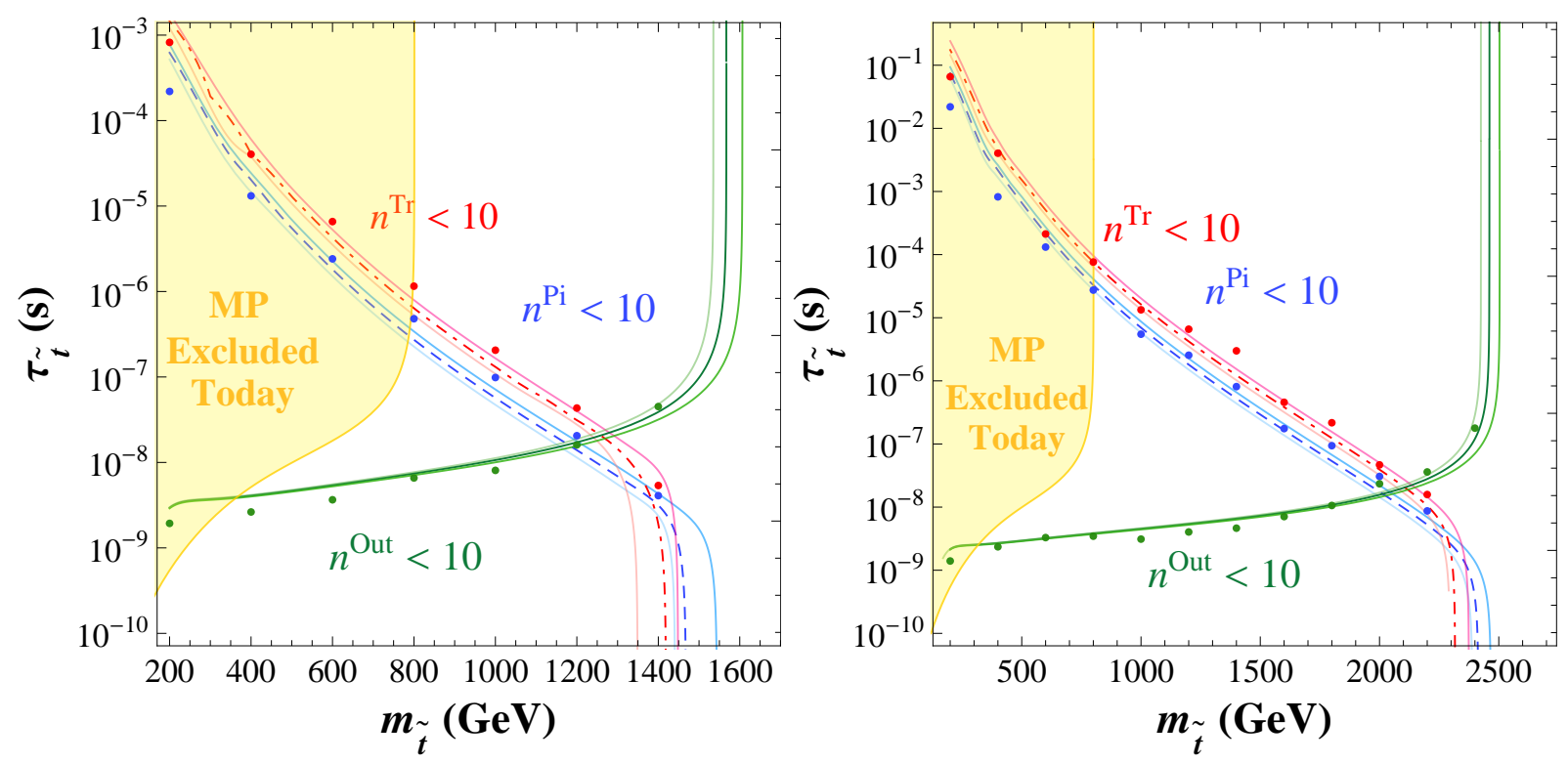

FiguRE 3.13: NLO LHC reach in the stop lifetime-stop mass plane at $L=25(3000) \mathrm{fb}^{-1}$ in the left (right) panel. The centre of mass energy is $\sqrt{s}=14 \mathrm{TeV}$ in both panels. In both panels, the MadGraph-Pixel reach is denoted by blue points and its corresponding semi-analytical one by a blue dashed line. The MadGraph-Tracker reach instead is denoted by red points and its corresponding semi-analytical one by a red dot-dashed line. The MadGraph-reach of metastable particles is denoted by green points and its corresponding semi-analytic one by a green solid line. In the upper left corner of each panel, the current excluded region for metastable particles $(M P)$ is painted yellow. Each of the original analytical curves has their own uncertainty of $\pm 1 \sigma$, denoted by two new analytical curves which draw a region around the original curve for $n=10$.
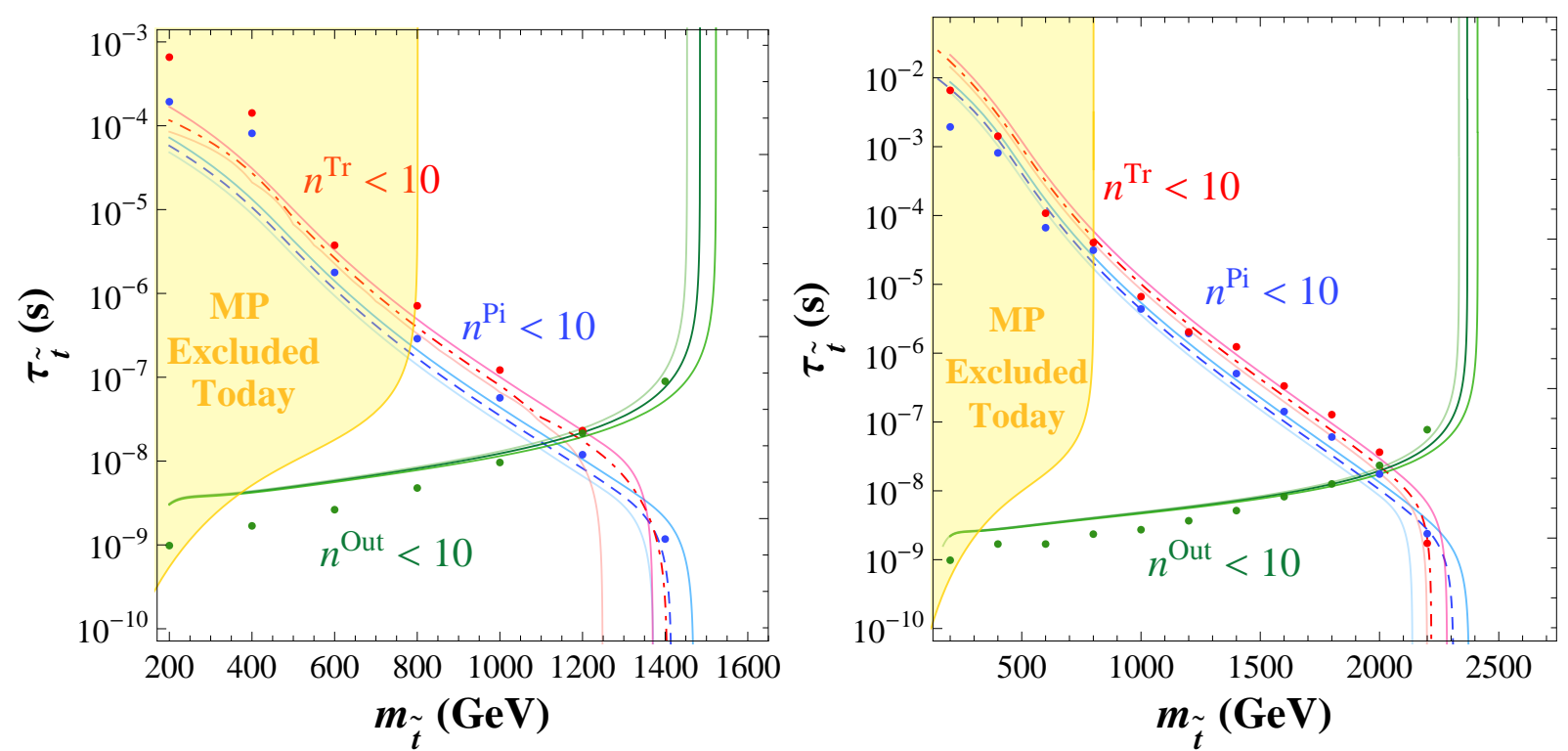

Figure 3.14: $L O L H C$ reach in the stop lifetime-stop mass plane at $L=25(3000) \mathrm{fb}^{-1}$ in the left (right) panel. For the description of this figure, see caption of Figure 3.13.

region we have drawn is achieved for a reheating temperature of $T_{R}=10^{3}$ and $10^{7} \mathrm{GeV}$ and a ratio of physical gaugino masses to stop mass of $M_{i} / m_{\tilde{t}}=1.1$. The left panel of the Figure 3.15 shows the LHC reach for the RPC stop decay for an integrated luminosity of $L=25 \mathrm{fb}^{-1}$ while the right panel for $L=3000 \mathrm{fb}^{-1}$. We note that the cosmological allowed region (white region) 

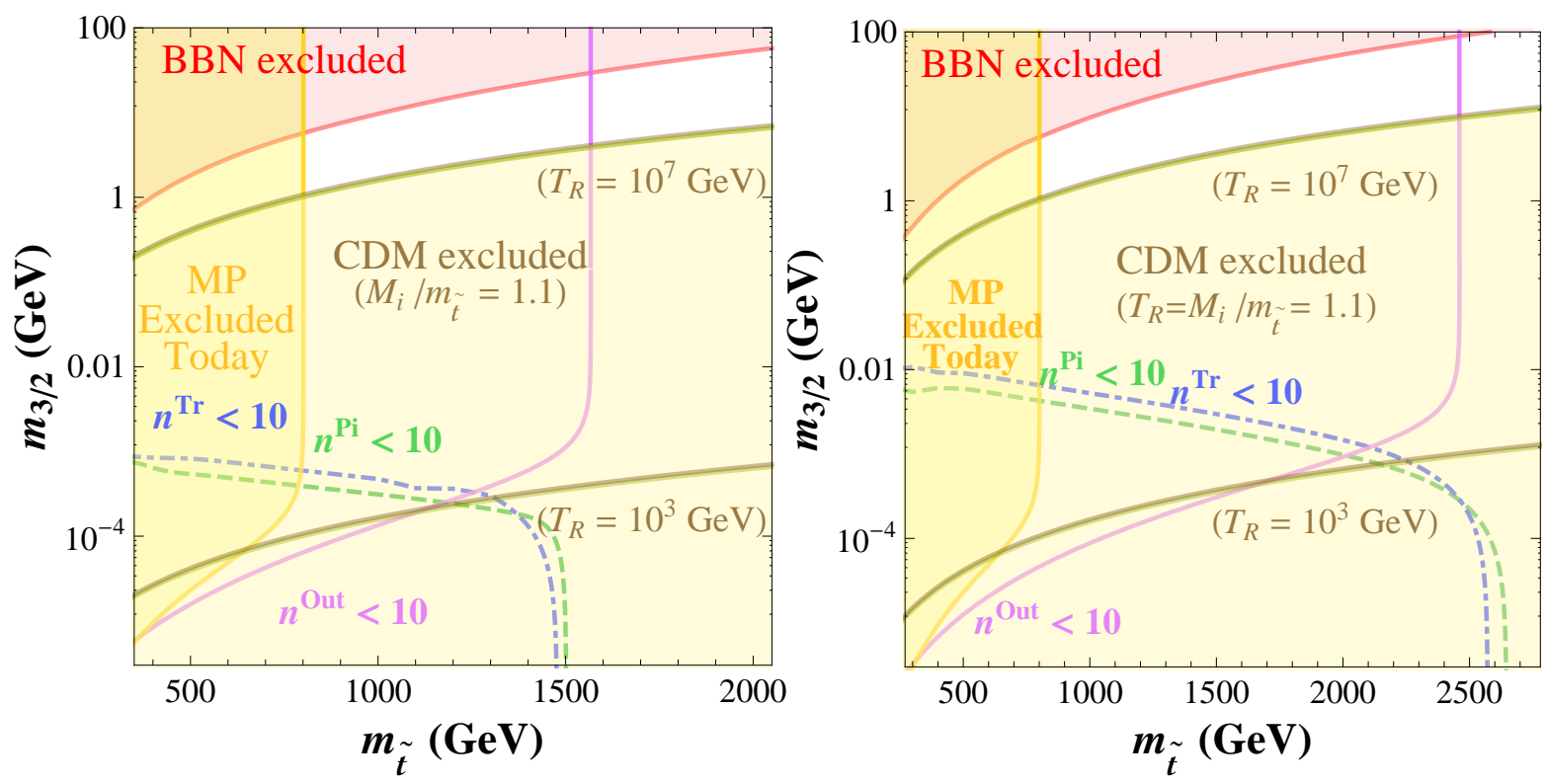

FiguRE 3.15: LHC reach in the stop mass-gravitino mass parameter space at NLO for the $R P C$ decay $\tilde{t} \rightarrow \psi_{3 / 2}$ t for $25 \mathrm{fb}^{-1}$ (left panel) and for $3000 \mathrm{fb}^{-1}$ (right panel) luminosity. The centre of mass energy is $\sqrt{s}=14 \mathrm{TeV}$ in both of them. On the left side of each panel the current excluded region for metastable particles (MP) is tinted yellow. The analytical curves that correspond to 10 displaced vertices in Pixel, Tracker and outside the detector are denoted by a dashed green line, a dot-dashed blue line and a solid pink line respectively. The BBN and $C D M$ excluded regions are also painted red and yellow each in order. Particularly, the latter has been obtained for $T_{R}=10^{3}$ and $10^{7} \mathrm{GeV}$.

appears above all of three detector curves, which means that stop NLSPs with a consistent cosmology and high reheat temperature can be only detected as metastable particles. The detection of a displaced vertex would instead point to the case of small gravitino mass and low reheating temperature.

\section{LHC reach for the RPV stop decay}

For the bilinear RPV model, the LHC reach can be reformulated in the R-parity breaking parameter-stop mass plane $\epsilon$ vs $m_{\tilde{t}}$ by using the analytic formula of the bilinear RPV stop lifetime given by Equation (3.8).

In Figure 3.16 the approximated curves at NLO for Pixel, Tracker and the part outside the detector are represented by a dashed blue line, a dot-dashed red line and a solid green line, respectively. We take here a parameter point with $\sin \theta / \cos \beta=0.017$, corresponding to relatively small stop mixing. For larger stop mixing the lifetime of the stop becomes shorter and therefore the gap between the metastable particles bound at small $\epsilon$ and the Dark Matter indirect detection bound increases.

On the left panel the LHC reach for $L=25 \mathrm{fb}^{-1}$ is shown whereas on the right panel for $L=3000 \mathrm{fb}^{-1}$. Here we also display the corresponding MadGraph 5 data by points which are blue for Pixel, red for Tracker and green for the decays that occur outside CMS.

At the lower left-hand corner of each plots the current excluded region for metastable particle (MP) is painted yellow. At the top of each panel, on the other hand, we see the indirect detection excluded region for the gravitino dark matter decay. The gravitino decay, indeed, 

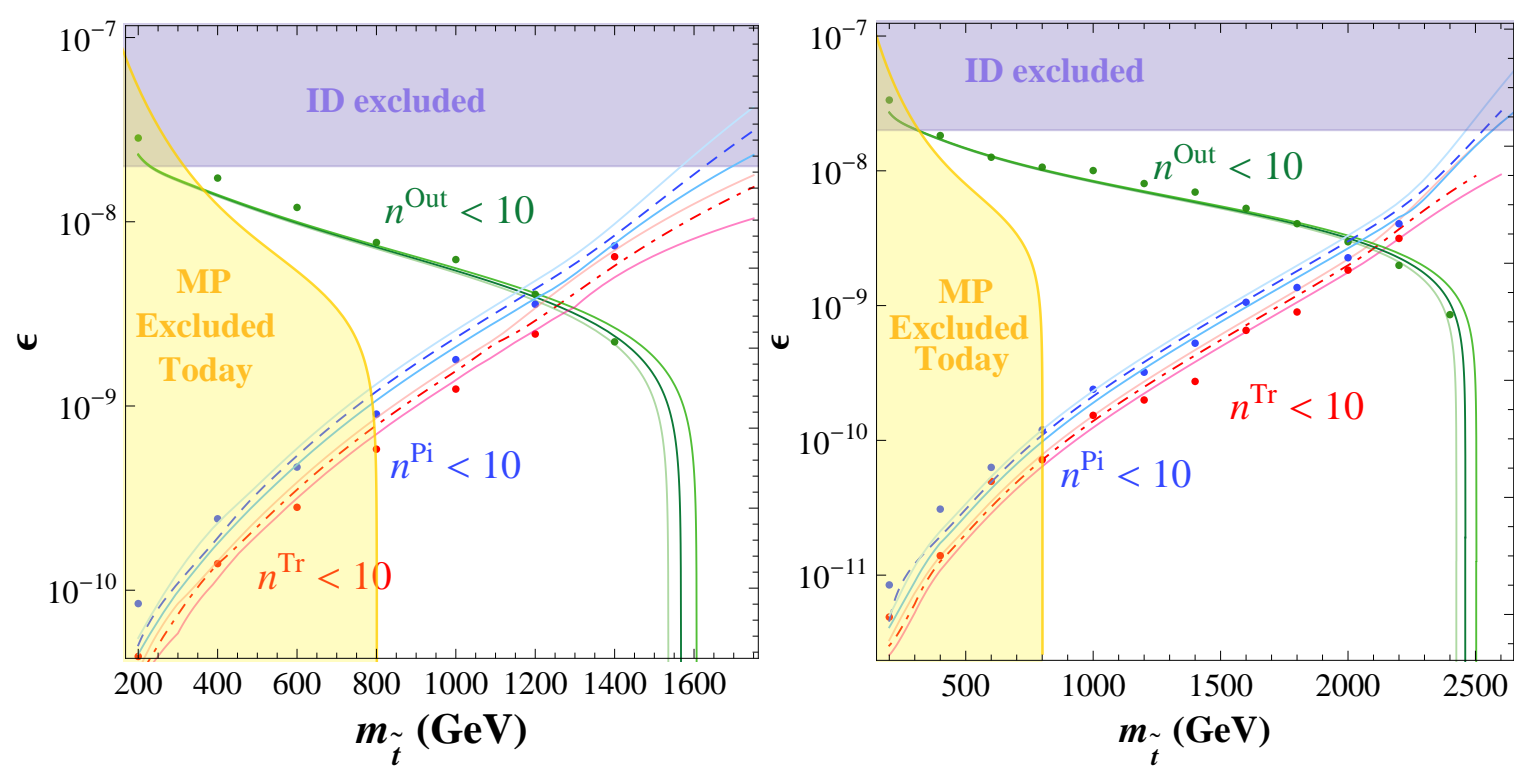

FIGURE 3.16: Reach in the R-Parity breaking parameter-stop mass plane for the RPV decay $\tilde{t} \rightarrow \ell^{+} b$ for $25 \mathrm{fb}^{-1}$ (left panel) and for $3000 \mathrm{fb}^{-1}$ (right panel) including the NLO correction to the production cross-section. Around each of the original analytical curves for 10 events, we show also the curves corresponding to a fluctuation $\pm 1 \sigma$, i.e. 8 and 12 events, as thinner lines. At the lower left corner of each panel the current excluded region for metastable particles (MP) is tinted yellow. On the top of each panel, instead, the indirect detection excluded region for the gravitino DM decay by Fermi-LAT collaboration is painted purple.

leads to a diffuse $\gamma$-ray flux which can be compared to the $\gamma$-ray flux observed by Fermi-LAT collaboration $[165,166]$ so as to get a severe lower bound on the gravitino lifetime, and therefore a upper bound on the R-parity breaking parameter $\epsilon$. Note that the latter bound depends on the gravitino mass, but not on the stop mass. Particularly, we take here the value of the upper bound on the R-parity breaking parameter $\epsilon$ to be $\epsilon \simeq 2 \times 10^{-8}$ for a gravitino mass of the few GeVs from [167].

We see in this case that the analysis of displaced vertices is absolutely needed to close the gap between the indirect detection bound and the possible metastable particle constraint. Indeed in this case both displaced vertices and metastable stops can be a signature in the cosmologically favorable region. Let us conclude this discussion pointing out that the majority of the parameter space is not excluded by the current indirect detection upper bound on the R-parity breaking parameter for a decaying DM gravitino and neither by the current excluded region for metastable particles. The LHC experiment will be able in the near future to explore all the parameter space up to the point where the stop NLSP is too heavy to be produced in sufficient numbers.

\subsection{RPC and RPV stop NLSP decays at LHC}

In the previous section we have performed a decay-channel independent analysis, relying only on the presence or absence of a displaced vertex in the CMS detector. But in case displaced vertices are observed with an antilepton and a bottom quark-jet in the final state, the question that arises is which model between the two we are considering here is realized. In Figure 3.17 and Figure 3.18 we show the Feynman diagrams for the two stop decay channels in the RPV and RPC models. They correspond to a 2-body and a 4-body decay with the same visible particles, 


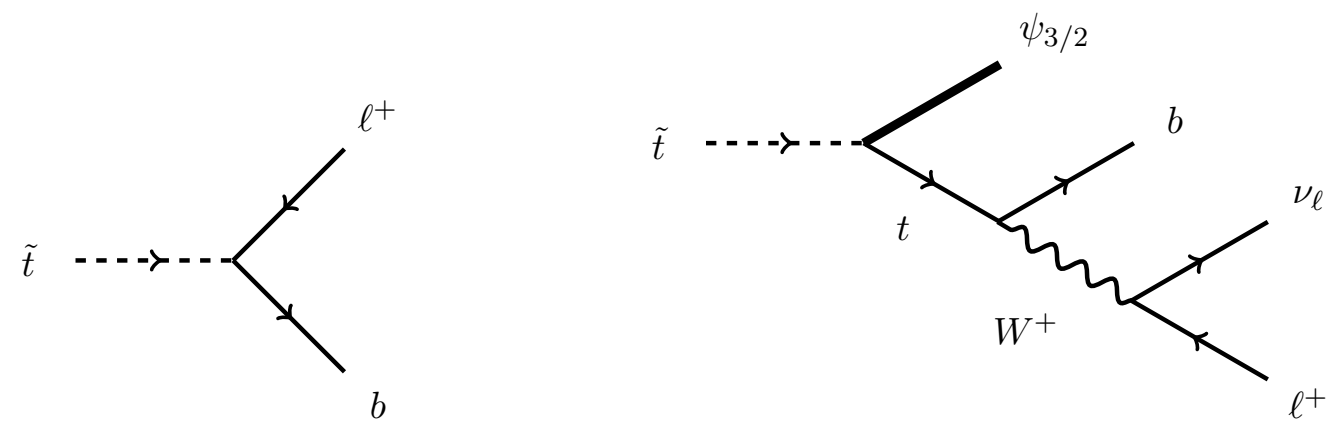

Figure 3.17: 2-body RPV stop decay.

Figure 3.18: 4-body RPC stop decay.

a $b$-quark and charged lepton, i.e.

$$
\begin{gathered}
\tilde{t} \rightarrow b \ell^{+} \\
\tilde{t} \rightarrow t \psi_{3 / 2} \rightarrow W^{+} b \psi_{3 / 2} \rightarrow b \ell^{+} \nu_{\ell} \psi_{3 / 2} .
\end{gathered}
$$

Fortunately they can be distinguished by the missing energy in the decay and the particle kinematics.

The different phase space of these decay channels can be observed in different kinematical variables related to the visible particles. In particular, we focus our attention on the three following observables in the laboratory frame of reference: the antilepton transverse momentum $P_{\ell T}$, the transverse mass of the pair antilepton-bottom $M_{T}$ and finally, the angle between the bottom and the antilepton momentum $\theta_{\ell b}$, which are defined by the formulas:

$$
\begin{gathered}
P_{\ell T}=\sqrt{p_{\ell x}^{2}+p_{\ell y}^{2}}, \\
M_{T}=\sqrt{\left(E_{\ell}+E_{b}\right)^{2}-\left(p_{\ell T}+p_{b T}\right)^{2}}, \\
\theta_{\ell b}=\arccos \left(\frac{p_{\ell x} p_{b x}+p_{\ell y} p_{b y}+p_{\ell z} p_{b z}}{\sqrt{p_{\ell x}^{2}+p_{\ell y}^{2}+p_{\ell z}^{2}} \sqrt{p_{b x}^{2}+p_{b y}^{2}+p_{b z}^{2}}}\right) .
\end{gathered}
$$

The variables $E_{\ell}, \mathbf{p}_{\ell}=\left(p_{\ell x}, p_{\ell y}, p_{\ell z}\right), E_{b}, \mathbf{p}_{\mathbf{b}}=\left(p_{b x}, p_{b y}, p_{b z}\right)$ stand for the energy and the three-momentum of antilepton and bottom, respectively.

In order to compare these kinematical quantities for the two decay channels of the stop, we simulate both processes with MadGraph 5 for a stop mass of $m_{\tilde{t}}=800 \mathrm{GeV}$ and the same stop decay rate of $\Gamma_{\tilde{t}}=2.02159 \times 10^{-10} \mathrm{GeV}$. As regards these simulations, we point out that since the RPV stop decay, differently from the RPC stop decay (see Appendix C), was not implemented in Madgraph, we had to directly implement it. Luckily, the RPV vertex under consideration only consisted of few lines of code and therefore we added them to the code related to all vertices of MSSM. To this aim we needed the package FeynRules so as to generate the model which could be used by Madgraph. In appendix $\mathrm{C}$ we show the above-mentioned lines of code and explain more in detail the procedure to make Madgraph run our RPV stop decay.

The transverse momentum distribution of the final antilepton for the two-body stop decay and the four-body stop decay are displayed in the left and the right panel of Figure 3.19 respectively. By looking at this figure we see that the peak of the two-body distribution is located at a much larger transverse momentum than the peak of the four-body distribution, because in the two body decay the lepton takes away around half the rest energy of the stop. Note that 

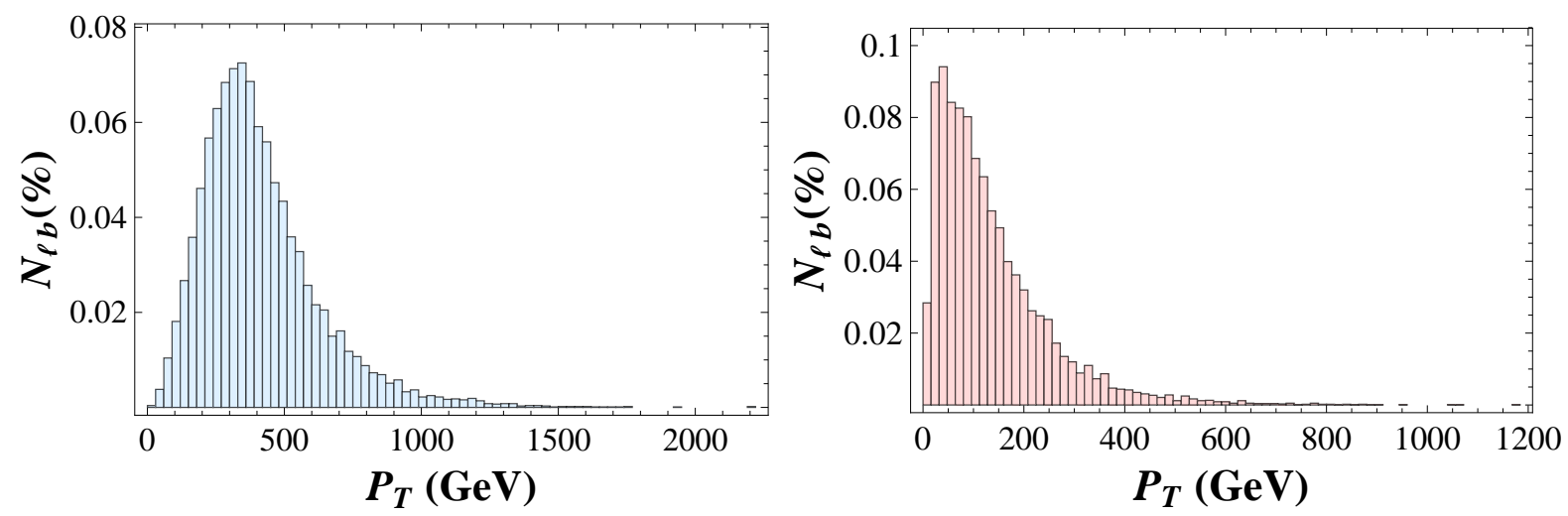

FIGURE 3.19: Transverse momentum distribution of the final antilepton for the two-body RPV $\tilde{t}$ decay (left) and four-body RPC $\tilde{t}$ decay (right) at $m_{\tilde{t}}=800 \mathrm{GeV}$.

the presence of a lepton or an antilepton in the final state is very useful since it provides a clear and robust signature in the detector, which significantly suppresses the large Standard Model background (e.g. QCD). In addition, the measurement of light leptons at CMS is more precise than that of jets.

The transverse mass distribution of the final pair antilepton-bottom for the two-body stop decay and the four-body stop decay are plotted in the left and the right panel of Figure 3.20 respectively. As we can see from the figure, the two distributions are very different. The twobody distribution has a peak and endpoint at a transverse mass of $M_{T}=800 \mathrm{GeV}$ corresponding to the stop mass, while the four-body distribution shows no peak and tends to be concentrated to a much smaller mass range. The difference is due to the missing energy in the four-body decay, while the two-body decay distribution allows to infer the stop mass from the position of the endpoint. The angular distribution between the final bottom momentum and the final antilepton momentum for the two-body stop decay and the four-body stop decay are displayed in the left and the right panel of Figure 3.21 respectively. We see in this figure that both distributions have no real peak, but they are centered at different ranges of angle $\theta_{\ell b}$, which are $\pi / 2 \lesssim \theta_{\ell b} \lesssim 3 \pi / 4$ for the two-body decay and $\theta_{\ell b} \lesssim \pi / 3$ for the four-body decay. This again is consistent with the fact that in the two-body decay the lepton and bottom are back-toback in the (slowly-moving) stop rest system, while in the four-body decay they recoil against a non-negligible missing momentum. Note that the distributions we have just displayed are independent from the reference decay rate we have chosen and are valid also for different values of $\Gamma$. In general these distributions can be used not only to distinguish between the two RPC and RPV decays discussed here, but also to disentangle the signal from the SM background from top decays and from the case of a different model or LSP, e.g. to compare to the case of a right-handed sneutrino LSP [168] or to other models like the $\mu \nu \operatorname{MSSM}[127,169]$.

\section{Background and coincidence counting}

So far we have completely neglected the background of Standard Model and Supersymmetric particles, a clearly optimistic working hypothesis. The most important SM background comes from the top-pair production at LHC. In fact, if the top quark decays into $W^{+}$and bottom $b$ and at last, the weak boson $W^{+}$decays into antilepton $\ell^{+}$and neutrino $\nu_{\ell}$, we obtain in the final state the same visible particles as in the RPC and RPV stop decay. On the other hand, the 

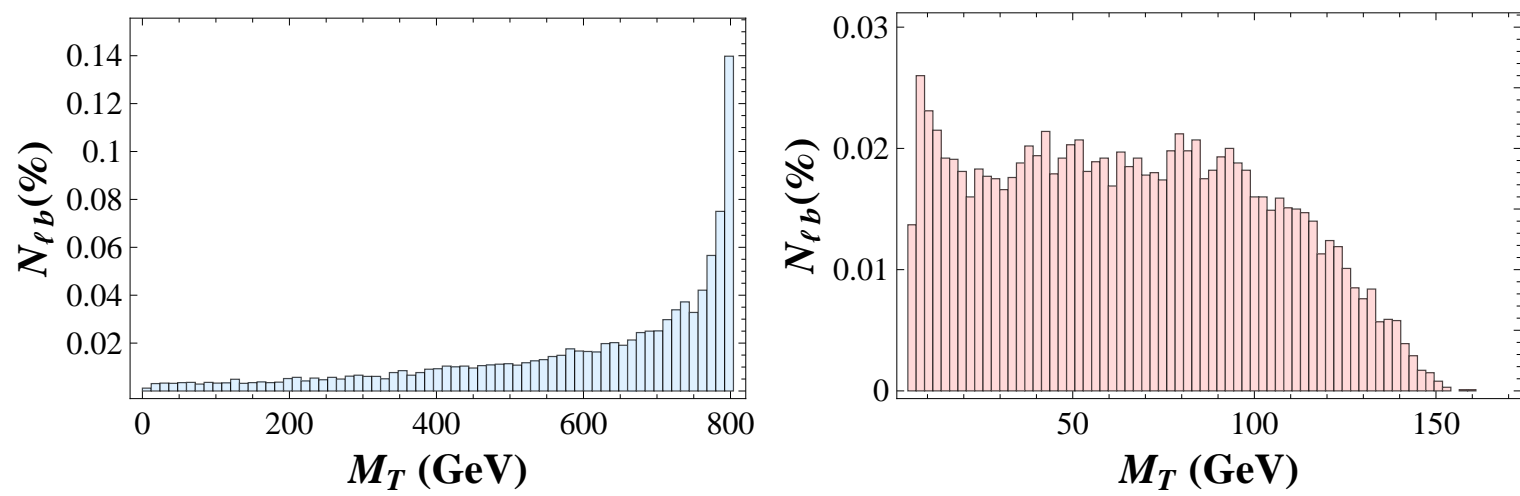

FiguRE 3.20: Transverse mass distributions of the final pair antilepton-bottom for the two-body $R P V \tilde{t}$ decay (left) and the four-body RPC $\tilde{t}$ decay (right) at $m_{\tilde{t}}=800 \mathrm{GeV}$.
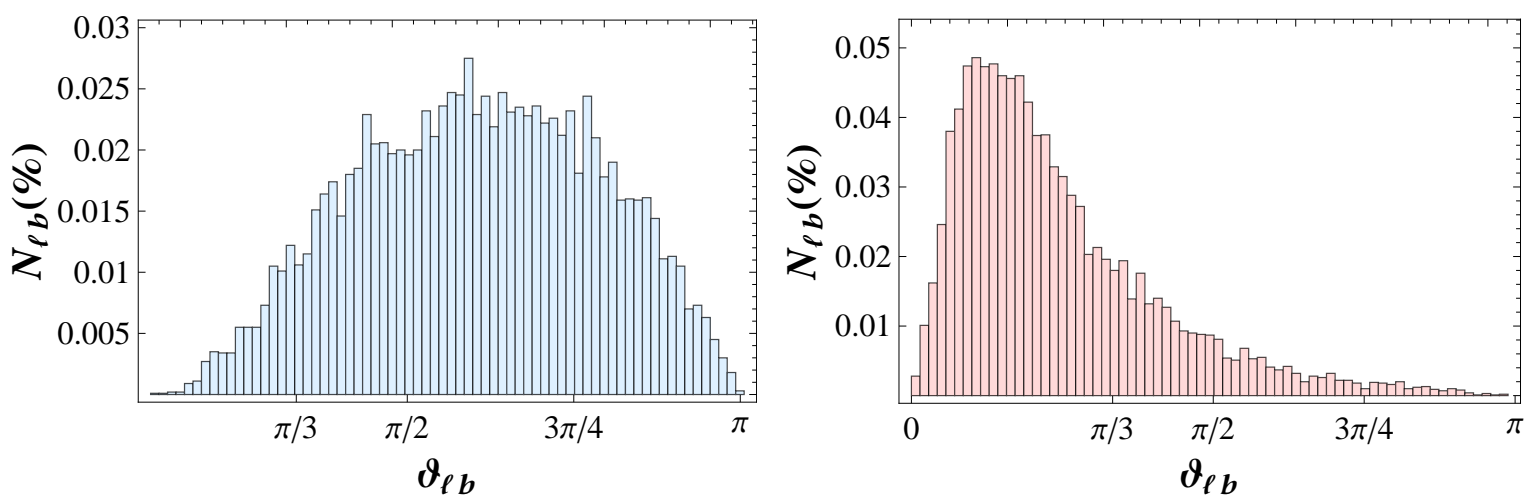

FIGURE 3.21: Angle distributions of the final pair antilepton-bottom for the two-body RPV $\tilde{t}$ decay (left) and four-body $R P C \tilde{t}$ decay (right) at $m_{\tilde{t}}=800 \mathrm{GeV}$.

top decays even before hadronizing and much faster that the stop discussed here and therefore one can avoid all this SM background just by requiring a displaced vertex. More difficult is to eliminate another source of background coming from $b \bar{b} Z \rightarrow b \bar{b} \ell^{+} \ell^{-}$, where the $b$-decay happens naturally away from the primary vertex and the lepton tracks are mis-reconstructed, as originating away from the interaction point. Moreover also underlying events can give rise to particles pointing to a secondary vertex, faking the presence of a long-lived particle.

In general a good strategy to eliminate such kind of reducible background is to consider the presence of two displaced vertices in the same event, both consistent with the same decay time, namely, the coincidence counting. Indeed we can see that in many cases one expects to have both stop and antistop to decay in the same part of the detector and give a clear signal for the production of two long-lived particles. Specifically, we give in Table 3.1 and Table 3.2 the percentage of decays of the stop and antistop in the different detector parts.

Particularly, we see from Table 3.1 and Table 3.2, that even in the unfortunate case of lifetime around $10^{-9} \mathrm{~s}$, which is at the boundary of the metastable particle searches, we have quite a large statistics of coincident events in the pixel and tracker, reaching approximately $50 \%$ of the displaced vertex events, irrespective of the stop mass. For longer lifetimes, the coincidence of two metastable particles in the same event takes over, reaching quickly a large statistics. 


\begin{tabular}{|c|c|c|c|c|c|c|}
\hline \multicolumn{7}{|c|}{$m_{\tilde{t}}=800 \mathrm{GeV} \quad \& \quad \tau_{\tilde{t}}=3.254 \times 10^{-9} \mathrm{~s}$} \\
\hline$\hat{t} \tilde{t}^{*}$ & Bp & $\mathrm{Pi}$ & $\operatorname{Tr}$ & $\mathrm{Ib}$ & Out & Tot \\
\hline$\overline{B p}$ & $3.29 \%$ & $5.90 \%$ & $3.64 \%$ & $1.20 \%$ & $0.02 \%$ & $14.05 \%$ \\
\hline $\mathrm{Pi}$ & $5.74 \%$ & $17.13 \%$ & $11.75 \%$ & $5.08 \%$ & $0.00 \%$ & $39.70 \%$ \\
\hline $\operatorname{Tr}$ & $3.15 \%$ & $11.98 \%$ & $10.01 \%$ & $5.05 \%$ & $0.05 \%$ & $30.24 \%$ \\
\hline $\mathrm{Ib}$ & $1.21 \%$ & $5.09 \%$ & $5.61 \%$ & $3.89 \%$ & $0.02 \%$ & $15.82 \%$ \\
\hline Out & $0.03 \%$ & $0.06 \%$ & $0.05 \%$ & $0.05 \%$ & $0.00 \%$ & $0.19 \%$ \\
\hline Tot & $13.42 \%$ & $40.16 \%$ & $31.06 \%$ & $15.27 \%$ & $0.09 \%$ & $100 \%$ \\
\hline \multicolumn{7}{|c|}{$m_{\tilde{t}}=800 \mathrm{GeV} \quad \& \quad \tau_{\tilde{t}}=3.254 \times 10^{-7} \mathrm{~s}$} \\
\hline & Bp & $\mathrm{Pi}$ & $\operatorname{Tr}$ & $\mathrm{Ib}$ & Out & Tot \\
\hline $\mathrm{Bp}$ & $0.00 \%$ & $0.00 \%$ & $0.00 \%$ & $0.02 \%$ & $0.18 \%$ & "0.20\% \\
\hline $\mathrm{Pi}$ & $0.00 \%$ & $0.01 \%$ & $0.01 \%$ & $0.10 \%$ & $0.77 \%$ & $0.89 \%$ \\
\hline $\operatorname{Tr}$ & $0.00 \%$ & $0.04 \%$ & $0.05 \%$ & $0.26 \%$ & $1.41 \%$ & $1.76 \%$ \\
\hline $\mathrm{Ib}$ & $0.01 \%$ & $0.09 \%$ & $0.17 \%$ & $1.39 \%$ & $8.95 \%$ & $10.61 \%$ \\
\hline Out & $0.14 \%$ & $0.73 \%$ & $1.56 \%$ & $8.63 \%$ & $75.48 \%$ & $86.54 \%$ \\
\hline Tot & $0.15 \%$ & $0.87 \%$ & $1.79 \%$ & $10.40 \%$ & $86.79 \%$ & $100 \%$ \\
\hline
\end{tabular}

TABLE 3.1: Coincidence counting for stop and antistop for a stop mass of $m_{\tilde{t}}=800 \mathrm{GeV}$ and a stop lifetime of $\tau_{\tilde{t}}=3.254 \times 10^{-9}$ and $3.254 \times 10^{-7} \mathrm{~s}$. The labels $\mathrm{Bp}, \mathrm{P} i$, Tr, Ib, Out stand respectively for the part of CMS before Pixel, Pixel, Tracker, the part between Tracker and the end of CMS and the part outside CMS detector.

We can therefore conclude that requiring two coincident events does not reduce the signal statistics substantially, while it would certainly suppress the background from misidentification or underlying events.

\subsection{Discussion of results and conclusion}

We have studied in this paper the reach of the LHC in models with a stop NLSP and gravitino LSP, both for the case of R-parity conservation and violation. In both cases we expect the stop to have a long lifetime leading to the possibility of displaced vertices or metastable particles at the LHC.

From the cosmological perspective, in case of high reheat temperature above the electroweak scale, the RPC scenario seems to prefer gravitino masses above $1 \mathrm{GeV}$ and therefore lifetimes giving metastable stops, while for the RPV case only indirect detection of gravitino DM decay gives an upper bound on the RPV parameter $\epsilon$, still consistent with stop decays within the detector.

We have performed a model-independent analysis of the two expected signals: displaced vertices in pixel or tracker detectors and metastable tracks. Our analysis is based on the MadGraph event generator, which allows us to simulate both the stop-antistop production and its subsequent decays. We assumed here that most of the other colored supersymmetric particles are much heavier than the stop and outside of the LHC reach and therefore we considered only the stop direct production, computed by MadGraph at LO and then corrected to include NLO 


\begin{tabular}{|c|c|c|c|c|c|c|}
\hline \multicolumn{7}{|c|}{$m_{\tilde{t}}=2000 \mathrm{GeV} \quad \& \quad \tau_{\tilde{t}}=3.254 \times 10^{-9} \mathrm{~s}$} \\
\hline$\tilde{t} \tilde{t}^{*}$ & $\mathrm{Bp}$ & $\mathrm{Pi}$ & $\operatorname{Tr}$ & $\mathrm{Ib}$ & Out & Tot \\
\hline$\overline{\mathrm{Bp}}$ & $2.69 \%$ & $6.94 \%$ & $3.35 \%$ & $\bar{~} 0.78 \%$ & $0.01 \%$ & $13.77 \%$ \\
\hline $\mathrm{Pi}$ & $6.47 \%$ & $21.58 \%$ & $13.44 \%$ & $3.69 \%$ & $0.00 \%$ & $45.18 \%$ \\
\hline $\operatorname{Tr}$ & $3.00 \%$ & $13.10 \%$ & $10.22 \%$ & $3.95 \%$ & $0.00 \%$ & $30.27 \%$ \\
\hline $\mathrm{Ib}$ & $0.60 \%$ & $4.23 \%$ & $3.72 \%$ & $2.23 \%$ & $0.00 \%$ & $10.78 \%$ \\
\hline Out & $0.00 \%$ & $0.00 \%$ & $0.00 \%$ & $0.00 \%$ & $0.00 \%$ & $0.00 \%$ \\
\hline Tot & $12.76 \%$ & $45.85 \%$ & $30.73 \%$ & $10.65 \%$ & $0.01 \%$ & $100 \%$ \\
\hline \multicolumn{7}{|c|}{$m_{\tilde{t}}=2000 \mathrm{GeV} \& \tau_{\tilde{t}}=3.254 \times 10^{-7} \mathrm{~s}$} \\
\hline & $\mathrm{Bp}$ & $\mathrm{Pi}$ & $\operatorname{Tr}$ & $\mathrm{Ib}$ & Out & Tot \\
\hline $\mathrm{Bp}$ & $0.00 \%$ & $0.00 \%$ & $0.00 \%$ & $0.04 \%$ & $0.12 \%$ & "0.16\% \\
\hline $\mathrm{Pi}$ & $0.00 \%$ & $0.04 \%$ & $0.05 \%$ & $0.12 \%$ & $0.81 \%$ & $1.02 \%$ \\
\hline $\operatorname{Tr}$ & $0.00 \%$ & $0.02 \%$ & $0.04 \%$ & $0.20 \%$ & $1.91 \%$ & $2.17 \%$ \\
\hline $\mathrm{Ib}$ & $0.04 \%$ & $0.09 \%$ & $0.36 \%$ & $1.86 \%$ & $10.27 \%$ & $12.62 \%$ \\
\hline Out & $0.10 \%$ & $0.67 \%$ & $1.60 \%$ & $10.20 \%$ & $71.46 \%$ & $84.03 \%$ \\
\hline Tot & $0.14 \%$ & $0.82 \%$ & $2.05 \%$ & $12.42 \%$ & $84.57 \%$ & $100 \%$ \\
\hline
\end{tabular}

TABLE 3.2: Coincidence counting for stop and antistop for a stop mass of $m_{\tilde{t}}=2200 \mathrm{GeV}$ and a stop lifetime of $\tau_{\tilde{t}}=3.254 \times 10^{-9}$ and $3.254 \times 10^{-7} \mathrm{~s}$. The labels Bp, Pi, Tr, Ib, Out stand respectively for the part of CMS before Pixel, Pixel, Tracker, the part between Tracker and the end of CMS and the part outside CMS detector.

with a constant k-factor. For the decay length distribution in the detector we also devised a simple analytical estimate based on the decay formula and the stop momentum distribution, that matches very well the MadGraph data-points and allows for a much easier exploration of the parameter space.

We have seen that even for relatively long lifetimes a substantial number of decays can happen in the tracker or pixel parts of the CMS detector. They surprisingly present similar number of events and can both be exploited to measure the short lifetime region, while the metastable particle search extends the reach quite strongly at long lifetimes. We have therefore shown that the two search strategies are complementary and that both are needed in order to cover all the macroscopic stop lifetimes up to a certain stop mass. It is interesting to note that both searches, either displaced vertices or metastable states, run out of steam at a similar value of the stop mass, where the production cross-section becomes too small. In particular we obtain, neglecting the background, in the most conservative case a mass reach up to 1300 $\mathrm{GeV}$ and $2100 \mathrm{GeV}$ at NLO for LHC at $14 \mathrm{TeV}$ and with an integrated luminosity of $25 \mathrm{fb}^{-1}$ and $3000 \mathrm{fb}^{-1}$ respectively. As expected this reach is much larger than the minimal one for a metastable stau from Drell-Yan production as given e.g. in [35], but smaller to the reach expected for a metastable gluino. Of course a full detector simulation is needed to confirm our findings, but the prospects seem to be very favorable for a combined analysis of both displaced vertices and metastable particle searches as discussed here.

We have also estimated the effect of NLO corrections to the stop-production cross-sections and, eventually, found that such effects can not be neglected. As a consequence, the LHC mass 
reach extended by approximately $200 \mathrm{GeV}$ towards heavier stops compared to the LO results.

We have translated the LHC reach in the parameter space of two models with gravitino DM, either RPC or RPV. In the first model the region compatible with high reheating temperature leads only to the signal of HSCP and the observation of displaced vertices in the first run fase with $25 \mathrm{fb}^{-1}$ would directly point to $T_{R}<10^{4}-10^{3} \mathrm{GeV}$. In the second model instead the search for displaced vertices allows to close the gap between metastable particle and indirect detection bounds at low stop masses. In case displaced vertices are seen, the visible particle kinematics allows to distinguish between RPC and RPV decays from the presence or absence of any missing energy. The distributions and the flavour of the final lepton, together with the value of the stop lifetime will be crucial to disentangle the particular model.

In general, due to the pair production of stop and antistop, we expect to see in a large fraction of the events the coincident presence of two displaced vertices or two metastable particles in the same event, allowing to disentangle the signal from reducible backgrounds connected to b-decays or misidentification of overlapping events. 


\title{
Chapter 4
}

\section{Minimal Decaying DM at LHC}

\author{
"It is contrary to reason to say that there is a vacuum \\ or a space in which there is absolutely nothing"
}

René Descartes

In this chapter we investigate possible LHC signature of the minimal decaying DM model, introduced in Section 1.4 of Chapter 1 and recently proposed in [8]. In this simplified model for DM particles we only have two additional states (a DM Majorana fermion and a colored or electroweakly charged scalar) with respect to the SM particle spectrum. All the coupling terms of the two new fields with SM particles are also renormalizable. The simple model so obtained can be embedded in more complicated and richer models (e.g. R-parity violating SUSY models) acting as their low-energy limit. As long as the rest of states and the other couplings are outside the LHC reach, our model can indeed have the same key features of those elaborate models.

These interactions allow to produce the right amount of DM in the early Universe through the decay of the charged heavy scalar, either in-equilibrium (Freeze-in) or out-of-equilibrium (SuperWIMP). At the same time, these interactions also allow to open up new decay channels for DM, which can nevertheless be sufficiently long-lived to be a good DM candidate and within reach of future Indirect Detection observations.

Due to the tiny value of the involved couplings, the expected signature at LHC of this model consists again in long-lived particles (i.e. long-lived scalars), which can again show up either as displaced vertices or as metastable particles. Therefore, in order to obtain their LHC reach we apply the method introduced in the previous chapter. Our hope is to pinpoint a parameter region where the model is both cosmologically viable and observable via LHC signals and see the future sensitivity of the LHC experiment to this kind of decaying DM model.

Equipped with the theoretical and cosmological introduction for this minimal model, we organize this chapter as follows: firstly we study the LHC phenomenology in the scenario where the scalar field carries color charge. Secondly, we repeat the same investigation in the scenario of scalar field carrying only electroweak charge. Finally, Section 4.1 will be then devoted to the discussion of our result and the conclusions we can reach. 


\subsection{Collider analysis}

Contrary to DM, the scalar field $\Sigma_{f}$ is charged under SM gauge interactions which may give its efficient production at the LHC, if kinematically allowed. Since the Yukawa couplings with the quarks are much smaller than any of the gauge couplings, the main production channels at a proton-proton collider are gluon fusion into a scalar-antiscalar pair, for colored $\Sigma_{f}$, or Drell-Yan production, for the electroweakly or electromagnetically charged case. In either cases the production rate is practically independent on the details of the DM model, and given just by the mass and charge of the field $\Sigma_{f}$. We will estimate here the NLO production rates by computing the LO cross-section through the package MadGraph 5 [144] and correcting with a constant NLO $k$-factor, depending on the channel.

For any given assignment of its quantum numbers, the scalar particle features two kind of decay channels after its production (We are implicitly assuming that in the case of the $S U(2)$ doublets $\Sigma_{q}$ and $\Sigma_{\ell}$ the two components are mass degenerate. If this is not the case, in addition to the processes described below, the decay of the heavier component of the doublet into a $W$, either on or off shell, and the lighter one is open. We will better clarify this point later in the text). In the first $\Sigma_{f}$ decay channel, we have decays into a DM particle and a standard model fermion with rate proportional to $\lambda^{2}$. They can be classified, according to the effective lagrangians in (1.83), as follows:

$$
\begin{array}{lll}
\Sigma_{f} \rightarrow u \psi & \text { for } & \Sigma_{q}, \Sigma_{u}, \Sigma_{d} \\
\Sigma_{f} \rightarrow d \psi & \text { for } & \Sigma_{q}, \Sigma_{d} \\
\Sigma_{f} \rightarrow l \psi & \text { for } & \Sigma_{\ell}, \Sigma_{e} \\
\Sigma_{f} \rightarrow \nu \psi & \text { for } & \Sigma_{\ell}
\end{array}
$$

where $l$ is a charged lepton. We see that only in the case of $\Sigma_{\ell}$ the decay can be into an invisible final state $\nu \psi$, but in that case also the visible channel into a charged lepton is present. So the decays in general give rise to a kink in the observable charged track/jet due to the $\Sigma_{f}$ decay.

The scalar field can decay as well into two SM fermions, with a rate governed by $\lambda^{\prime 2}$, according the following channels:

$$
\begin{aligned}
& \Sigma_{f} \rightarrow q \bar{q}^{\prime} \text { for } \quad \Sigma_{u}, \Sigma_{d}, \Sigma_{\ell} \\
& \Sigma_{f} \rightarrow q l \text { for } \quad \Sigma_{q}, \Sigma_{d} \\
& \Sigma_{f} \rightarrow q \nu \text { for } \quad \Sigma_{q}, \Sigma_{d} \\
& \Sigma_{f} \rightarrow l \bar{l} \text { for } \Sigma_{\ell} \\
& \Sigma_{f} \rightarrow l \nu \text { for } \Sigma_{\ell}, \Sigma_{e}
\end{aligned}
$$

where $l$ is, again, a charged lepton while $q$ is an up or down-type quark. In view of the dependence of the decay rate of $\Sigma_{f}$ on the $\lambda, \lambda^{\prime}$-type coupling, a tight relation exists between possible signals at LHC of such decays and the constraints from the DM phenomenology, being governed by the same couplings. As already argued in [8] the constraints from ID and from the cosmological abundance of the DM require very low values of the couplings, namely $\lambda, \lambda^{\prime} \lesssim 10^{-(7 \div 8)}$, thus implying that the decay vertices result displaced with respect to the production ones and may even lie outside the detector.

In order to determine the LHC capability of detecting this kind of decays we adopt the method introduced in the previous chapter. In other words we follow the same procedure, used 
in the previous chapter to find the LHC reach for the direct stop production. Thus we have generated several samples of pair produced $\Sigma_{f}$, at $14 \mathrm{TeV}$ of centre of mass energy, corresponding to different assignments of its quantum numbers and different masses, and determined the spatial distribution of the decay vertices from the kinematic variables of the events and the decay rate $\Gamma_{\Sigma}$ as described in more detail in the preceding chapter. Note that we consider here a straightline motion of the particle after production, neglecting the magnetic field deflection and the interactions with the intervening matter, which could even increase the number of decays in the inner part of the detector by bending the trajectory or slowing down the decaying particle ${ }^{1}$. Assuming $100 \%$ detector efficiency and no background, in order to claim the discovery for a given scenario we have required the presence of at least 10 decay events in one of the components in which the detector is schematized, i.e. in Pixel or Tracker, or outside the detector.

This is actually a rather conservative choice. Indeed 5 events are enough for a discovery at the $95 \%$ CL, assuming a pure Poisson distribution for the events and absence of background. On the other hand our more stringent requirement prevents the effects of statistical fluctuations, ensures stable numerical results and possibly accounts for a reduction of the signal efficiency once detector effects and eventual analysis cuts are considered (see e.g. [170]). We also remark that recent searches of displaced vertices have determined very low [170] or even null [171] expected background events. On the other hand these rely on some specific requirements, like the presence of a high $p_{T}$ final state muon in [171], which are not necessarily always fulfilled in our scenario.

The optimal scenario, and thus main focus of our analysis, is however a "double" LHC detection scenario, consisting in the contemporary detection of at least 10 events in one of the components of the inner detector, namely pixel or tracker, and 10 tracks leaving the detector. This indeed would allow for a cross-check in the measurement of the lifetime of the scalar particle as well as a better discrimination of possible backgrounds. For each of the cases considered we have performed the analysis for three definite luminosities, namely $25 \mathrm{fb}^{-1}, 300 \mathrm{fb}^{-1}$ and $3000 \mathrm{fb}^{-1}$, in order to determine the feasibility of a next future discovery during run II, as well as the maximal discovery reach considering the full LHC data set and, finally, after a high luminosity run.

Since the analysis employed is not sensitive to the particular type of decay products of $\Sigma_{f}$, as long as a vertex (or kink) can be observed and happens in the detector, we will from now on refer to a schematic setup described by just four model parameters: the mass $m_{\Sigma_{f}}$ of the scalar field, the ratio $x=m_{\psi} / m_{\Sigma_{f}}$ and two couplings $\lambda$ and $\lambda^{\prime}$, representing, respectively the coupling (and thus decay) of $\Sigma_{f}$ with the DM and a SM fermions and with two SM fermions. We have as well considered an equivalent representation in terms of the $\Sigma_{f}$ lifetime and DM branching fraction, more directly connected to the phenomenological observables at the LHC, and also helpful to translate the results obtained in different particle physics setups. In either case we will find the region of parameter space where displaced vertices and/or metastable tracks may be seen and compare it to the cosmologically viable parameter space.

As will be discussed later, it would be very important, in order to relate a hypothetical LHC signal to the DM properties, to distinguish both the decay channels of $\Sigma_{f}$. A necessary condition for the identification of a particular channel is that the product of the total number of events times the corresponding branching fraction is large enough, in one of the detector regions where it is possible to observe the decay products of the scalar field. A proper determination

\footnotetext{
${ }^{1}$ Such effects could be captured only by a full detector simulation, which is beyond the scope of this thesis.
} 
of the number of events needed would require the full detector simulation, accounting for the capability of reconstruction of the various decay products.

In the following subsections we will investigate separately the scenarios of color and electroweakly charged scalar particle.

\subsubsection{Colored scalar}

The first case that we are going to consider is when the field $\Sigma_{f}$ carries color charge. Colored states are expected to be more efficiently produced at the LHC. For definiteness we will consider a $\Sigma_{d}$-type field in our analysis. As already mentioned, the $\Sigma_{f=q, u, d}$ pairs are produced through gluon fusion and thus the production cross section is substantially the same for the three kind of states, apart a possible enhancement in the case of $\Sigma_{q}$ because of multiplicity. We will indeed assume, for this scenario, that the two components of the doublet $\Sigma_{q}$ are exactly degenerate in mass. If this is not the case the heaviest state of the doublet could decay into the lightest one and a $\mathrm{W}$ boson, if kinematically possible, or two quarks or leptons (through an off-shell W). For mass splittings above $\sim 1 \mathrm{GeV}$ this decay channel has a branching fraction substantially equal to one and leads to prompt decays of the heavy state in case its production is accessible at the LHC. For sizable enough mass splittings, such that the momentum of decay products can survive analysis cuts (e.g. quality of signal requirements, background discrimination cuts), this decay can be detected and, then, the signals discussed in the following would result accompanied by prompt jets or leptons. On the other hand the required mass splitting would imply a sensitive suppression of the pair production of the heaviest states of the doublet and thus a small number of this kind of events. This might not be the case of the $\Sigma_{\ell}$-type particles, as will be clarified in the next subsection.

According to the method described above, we have generated samples of events of $\Sigma_{d}$ pair production through MadGraph $[144,172]$. The obtained LO $\Sigma_{d}$ production is then corrected with the same NLO k-factor, given by Prospino [161], used for the stop production $(k=1.6)$ because of the similarities with the supersymmetric stop case, we have studied in the previous chapter. From the determination of the NLO production cross-section, it is thus possible to infer a general upper limit on the LHC mass reach at a given luminosity $L$ by exploiting the relation $N_{\mathrm{ev}}=\sigma_{p p \rightarrow \Sigma_{d} \Sigma_{d}^{*}} L \geq 5$ (10 events correspond to 5 pair produced $\Sigma$ ), where $N_{\text {ev }}$ represents the number of produced pairs $\Sigma_{d} \Sigma_{d}^{*}$ irrespective of the position of the decay vertices. Particularly, for the luminosities considered in our analysis, the LHC mass reach ranges from around $1600 \mathrm{GeV}$ at $L=25 \mathrm{fb}^{-1}$ to a maximal value of $2200 \mathrm{GeV}$ corresponding to $\mathcal{L}=3000 \mathrm{fb}^{-1}$.

According to the numerical analysis of the previous chapter (see Section 3.3), we have then computed through MadGraph and Python the spatial distribution of the $\Sigma_{d}$ decay vertices and their lifetime $\tau_{\Sigma_{d}}$ for several values of $\Sigma_{d}$ mass $(800,1600$ and $2200 \mathrm{GeV}$ ) which correspond to 10 events inside Pixel, Tracker and outside the detector. Finally, we have reformulated the obtained lifetime values in the plane $\lambda-\lambda^{\prime}$ by using the Equation (1.87) and the fixed mass value.

We report two examples of results of our analysis in Figure 4.1 , for $m_{\Sigma_{d}}=800 \mathrm{GeV}$ and $\mathcal{L}=300,3000 \mathrm{fb}^{-1}$, where we have identified the region corresponding to more than 10 decay events in the pixel, tracker and outside the detector. We see that the contours run piecewise parallel to the axis, since in most of the parameter space one single coupling dominates the total decay rate. The detection regions for pixel and tracker are very similar, since the difference in volume is practically compensated by their comparable distance from the interaction point.

The searches for displaced vertices and particles escaping from the detector are highly complementary: the first has a maximal reach at low lifetimes, corresponding to high values of $\lambda, \lambda^{\prime}$, 

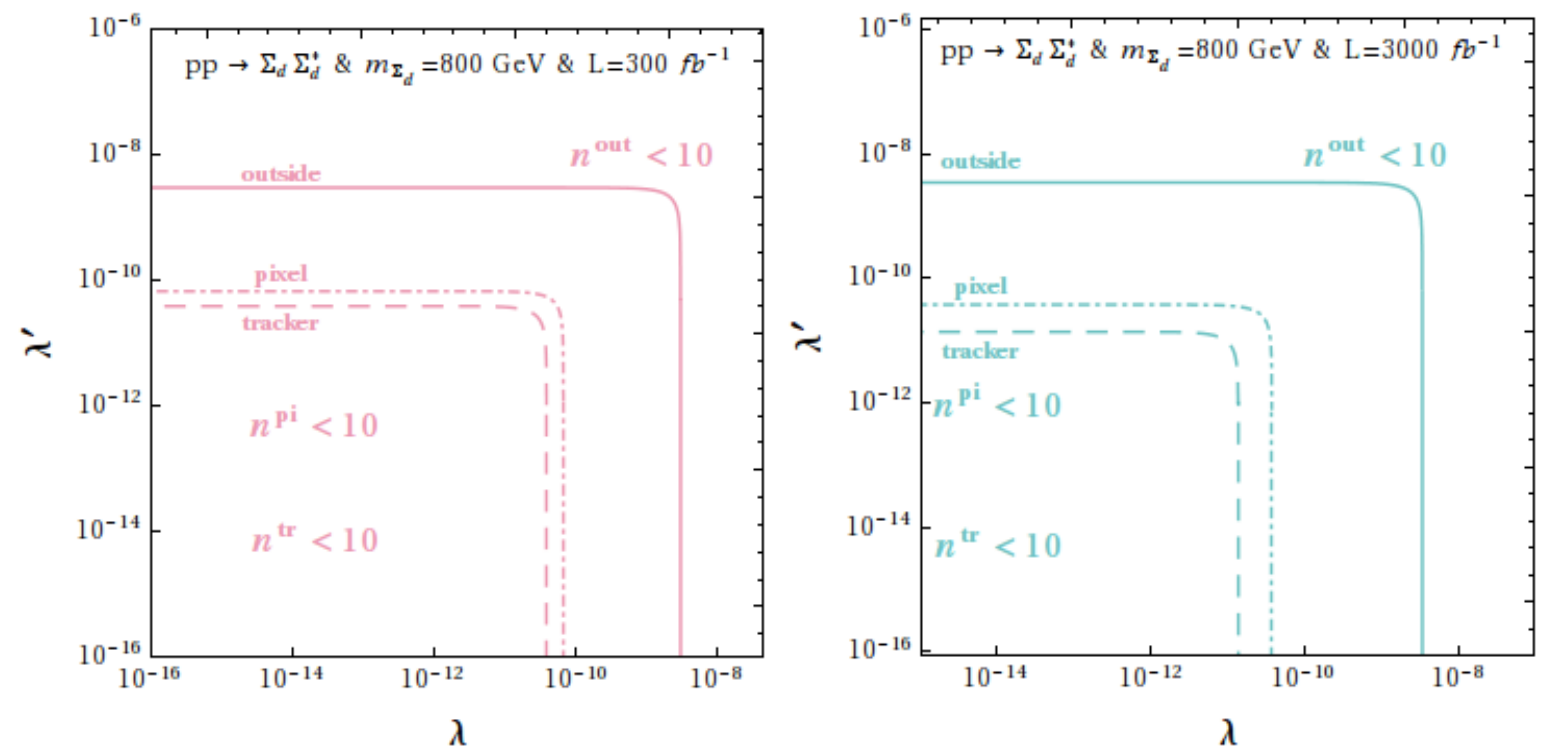

Figure 4.1: LHC reach in the plane of the pure $S M \lambda^{\prime}$ vs DM coupling $\lambda$ for (from left to right) $\mathcal{L}=300,3000 \mathrm{fb}^{-1}$ for $m_{\Sigma_{d}}=800 \mathrm{GeV}$. The region below the solid line corresponds to at least 10 metastable tracks, while the regions above the dashed/dash-dotted lines to at least 10 decay events in the tracker or pixel detector respectively.

whereas the latter is able to investigate efficiently the very long lifetimes, i.e. low values of $\lambda, \lambda^{\prime}$. Combining both search strategies allows to cover practically the whole parameter space.

The strong requirement of detection of both types of signal, i.e. displaced vertices in the pixel/tracker and metastable tracks, is realized only in the narrow regions comprised between the iso-contours in the plane $\left(\lambda, \lambda^{\prime}\right)$ representing the detection of exactly 10 events in the pixel, tracker and outside the detector. The size of these strips is expected to increase with the integrated luminosity and, instead, to shrink once increasing the mass of the scalar, because of the lower number of particles produced. For the highest values of the mass, corresponding to approximately $2200 \mathrm{GeV}$ the whole parameter space in the couplings might only be probed by the high luminosity upgrade of the LHC as long as both types of signal, either displaced vertex or metastable track, are considered.

The capability of LHC detection of a displaced decay of $\Sigma_{d}$ or its metastable track can be confronted with the requirement of the correct cosmological DM abundance via $\Sigma_{d}$ decay and, possibly, a detection of decaying DM. In case of a colored scalar the correlation between the DM phenomenology and the LHC predictions is rather straightforward since the DM production is substantially dominated by the first contribution in Equation (2.49), i.e. freeze-in contribution. Here, the coupling $\lambda$ :

$$
\lambda \simeq 1.59 \times 10^{-12} x^{-1 / 2}\left(\frac{g_{*}}{100}\right)^{3 / 4}\left(\frac{\Omega_{\mathrm{CDM}} h^{2}}{0.11}\right)^{1 / 2} g_{\Sigma}^{-1 / 2}
$$

can be analytically determined by using Equation (1.86) and (2.47), whereas the coupling $\lambda^{\prime}$ :

$$
\lambda^{\prime} \simeq 0.91 \times 10^{-12} x^{-2}\left(\frac{g_{*}}{100}\right)^{-3 / 4}\left(\frac{m_{\Sigma_{d}}}{1 \mathrm{TeV}}\right)^{-1 / 2} g_{\Sigma}^{1 / 2}\left(\frac{\tau_{\psi}}{10^{27} \mathrm{~S}}\right)^{-1 / 2}\left(\frac{\Omega_{\mathrm{CDM}} h^{2}}{0.11}\right)^{-1 / 2}
$$


can be instead determined by plugging the above expression for $\lambda$ into Equation (1.84). From these relations we can determine the cosmologically preferred parameter space in the plane of the couplings. We show indeed in Figure 4.2, as solid lines, the isolines of the correct value of the DM relic density for $m_{\Sigma_{d}}=800 \mathrm{GeV}$ and some values of $x$ ranging from $10^{-3}$ to 0.5 . They appear as vertical lines since the freeze-in mechanism is independent of the coupling $\lambda^{\prime}$. This curves can be confronted with the contours of the reach in the three detector regions. The panels of Figure 4.2 instead report the LHC reach for the three values of luminosity considered in our analysis. Figure 4.3 shows an analogous analysis for the two values of mass $m_{\Sigma_{d}}=\{1600,2200\}$ $\mathrm{GeV}$. In this case we have considered only $\mathcal{L}=3000 \mathrm{fb}^{-1}$ since we expect a statistically relevant number of events only for this very high luminosity.

In Figure 4.2 and 4.3 we have reported as well (dashed lines) the values of the DM lifetime, for the chosen combinations of parameters, near the present bounds. As already stated, we have assumed, for the scenario of colored scalar field, $u \bar{u}(d \bar{d}) \nu$ as the only relevant decay channel for the DM and thus applied the bounds of [42] in the $m_{\psi}>100 \mathrm{GeV}$ region and of [45] $]^{2}$ at lower masses. For any value of $x$, the intersection of the corresponding solid and dashed lines corresponds to a DM with the correct relic density and a lifetime approximately coinciding with the current observational bounds for the assumed dominant decay channel; consequently the parameter space above the DM lifetime curves may be already excluded. Notice that the actual ID exclusion region depends strongly on the DM decay channel and DM mass and is affected from astrophysical uncertainties in the propagation modeling as discussed in [42].

The most favorable scenario, consisting in a multiple detection of the DM and $\Sigma_{d}$ decays, respectively in cosmic rays and at the LHC, with the latter satisfying the double LHC detection requirement, is potentially feasible, for a given value of the pair $\left(x, m_{\Sigma_{d}}\right)$, when the corresponding isolines of the DM relic density and lifetime intersect inside the double detection region. Outside this region the contemporary ID of DM and only one type of LHC signal, i.e. displaced vertices (above the white strip) or metastable tracks (below the white strip), is anyway still feasible. We remark, however, that the region below the white strip, corresponding to very long lifetimes, is already constrained, for the lower values of the mass of the scalar, by current searches of detector stable particles. We have reformulated, for the scenario under consideration, these constraints by using the procedure described in the previous chapter, and eventually reported the excluded region in Figure 4.2. The region below the "double" detection strip is nearly ruled out for $m_{\Sigma_{d}}=800 \mathrm{GeV}$. The limit from detector stable particles weakens very quickly with increasing mass of the scalar particle and it is substantially irrelevant for masses above $1 \mathrm{TeV}$.

The double LHC detection region corresponds, for $m_{\Sigma_{d}}=800 \mathrm{GeV}$, to a rather definite range of values of $x$ comprised between $10^{-2}$ and $10^{-1}$. This range is reduced at higher values of $m_{\Sigma_{d}}$ because of the decreased size of the LHC double detection strip. For the highest possible value $m_{\Sigma_{d}}$ the combined detection prospects are substantially limited to $x \simeq 10^{-2}$.

It is also interesting to reformulate the previous results in a more model independent way in terms of the pair $\left(\Gamma_{\Sigma}^{-1}(\mathrm{~s}), \mathrm{BR}\left(\Sigma_{d} \rightarrow \psi f\right)\right)$ as done in Figure 4.4 and 4.5. Using these parameters the LHC detection regions are just delimited by horizontal lines of constant $\Gamma_{\Sigma}$ values. The green (violet) shaded regions in the plot represent the regions in which it is possible to detect more than 10 events in the pixel/tracker (outside region). "Double" signals are accessible instead in the middle white strip. The combined LHC detection of the $\Sigma_{d}$, in at least one of the two channels, and DM indirect detection are again achieved whenever the isolines (dash-dotted) of

\footnotetext{
${ }^{2}$ The bounds presented here actually refer to two body decays in fermion pairs and then result conservative since in our setup part of the energy of the products is carried away by the neutrinos.
} 

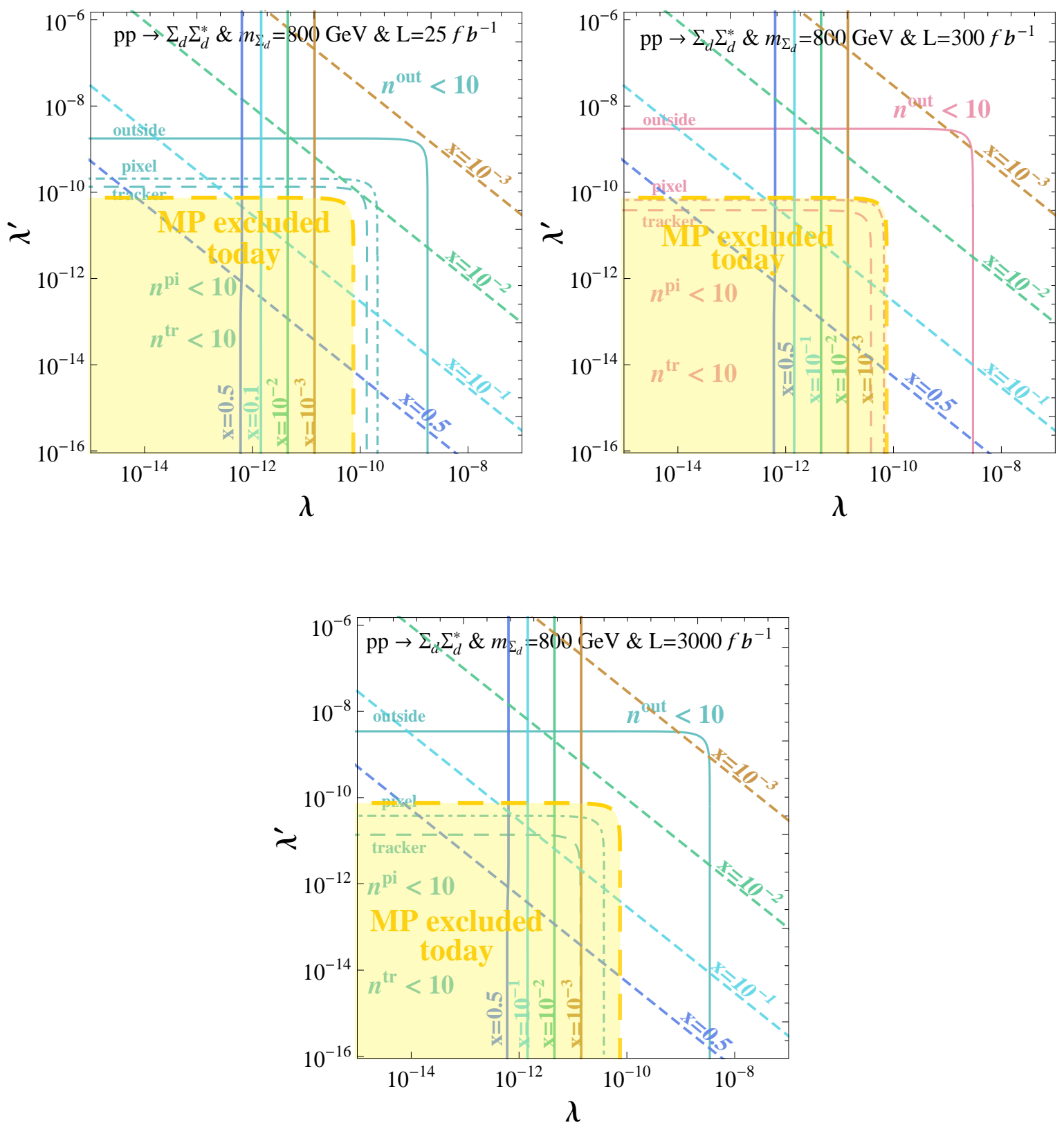

FIGURE 4.2: Contours of the correct DM relic density (solid lines) and of the reference value of $10^{28} \mathrm{~s}$ of its lifetime (dashed lines) color-coded according to the values of $x$ reported in the plot. A combined detection of $\Sigma_{d}$ at LHC and of the DM candidate via ID can be achieved if the lines of the DM relic density and lifetime, corresponding to a given value of $x$, intersect within the discovery region between the iso-contours labeled as pixel, tracker and outside. The plot refers to $m_{\Sigma_{d}}=800 \mathrm{GeV}$ and $\mathcal{L}=25 \mathrm{fb}^{-1}$ (left plot), $\mathcal{L}=300 \mathrm{fb}^{-1}$ (left plot), $\mathcal{L}=3000 \mathrm{fb}^{-1}$ (bottom plot). The yellow shaded region delimited by the yellow thick long-dashed line is already excluded by current searches for metastable particles.

the reference DM lifetime and of the correct relic density (solid) for a fixed value of $x$ cross in the LHC "double" discovery region. Above this strip it is still possible to observe displaced vertices at the LHC and have an ID DM signal for small values of $x$. At large values of $x$, instead, only metastable particle signals are compatible with DM ID. 

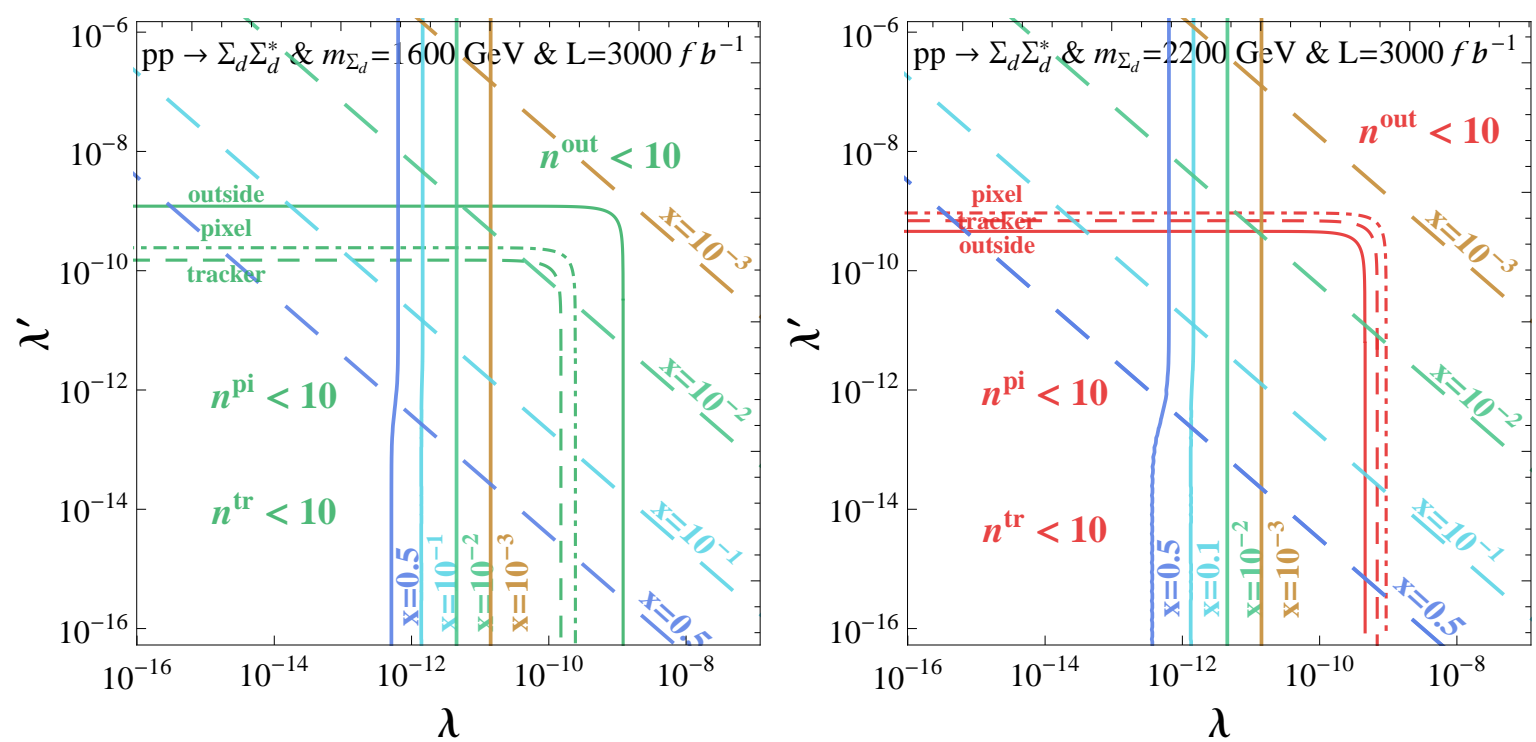

Figure 4.3: The same as fig. (4.2) but with $m_{\Sigma_{d}}=1600 \mathrm{GeV}$ (left plot) and $m_{\Sigma_{d}}=2200 \mathrm{GeV}$ (right plot) and $\mathcal{L}=3000 \mathrm{fb}^{-1}$ in both cases.

\begin{tabular}{|c|c|c|c|}
\hline Part of detector & Total & $\Sigma \rightarrow D M$ & $\Sigma \rightarrow$ SM only \\
\hline $\mathcal{L}=25 \mathrm{fb}^{-1}$ & \multicolumn{3}{|l|}{} \\
\hline pixel & 63 & 0 & 63 \\
\hline tracker $^{\mid}$ & 125 & 0 & 125 \\
\hline out & 907 & 1 & 906 \\
\hline $\mathcal{L}=300 \mathrm{fb}^{-1}$ & \multicolumn{3}{|l}{} \\
\hline pixel & 757 & 0 & 757 \\
\hline tracker $^{\mid}$ & 1504 & 2 & 1502 \\
\hline out & 10889 & 11 & 10878 \\
\hline $\mathcal{L}=3000 \mathrm{fb}^{-1}$ & \multicolumn{3}{|l}{} \\
\hline pixel & 7571 & 8 & 7563 \\
\hline tracker & 15043 & 15 & 15028 \\
\hline out & 108892 & 113 & 108779 \\
\hline
\end{tabular}

TABLE 4.1: Number of decay events, total as well as separately in the two kind of decay channels (DM or SM only), which is expected to be observed, in the three detection regions, at the indicated values of the luminosity, for a $\Sigma_{d}$ production scenario corresponding to the benchmark set of masses $m_{\Sigma_{d}}=800 \mathrm{GeV}$ and $m_{\psi}=10 \mathrm{GeV}$. The coupling $\lambda$ and $\lambda^{\prime}$ have been chosen in such a way that the DM achieves the correct relic density, through the freeze-in mechanism, and its lifetime is $10^{28} \mathrm{~s}$, just beyond present ID limits. The values of $\lambda$ and $\lambda^{\prime}$ are, respectively, $1.8 \times 10^{-11}$ and $5.5 \times 10^{-10}$.

\section{Benchmarks for colored $\Sigma_{f}$}

Simultaneous ID/LHC detection, with the latter in the form of a double signal, i.e. displaced decay plus metastable track, could be achieved in the next future, namely for luminosities up to $300 \mathrm{fb}^{-1}$, for values of $\operatorname{Br}\left(\Sigma_{d} \rightarrow d \psi\right)$ lower than $10^{-3}$. As a consequence the observation at the LHC of both the DM and only SM decay channels of $\Sigma_{d}$ appears difficult but it is not a priori excluded provided that there is a high statistics. 

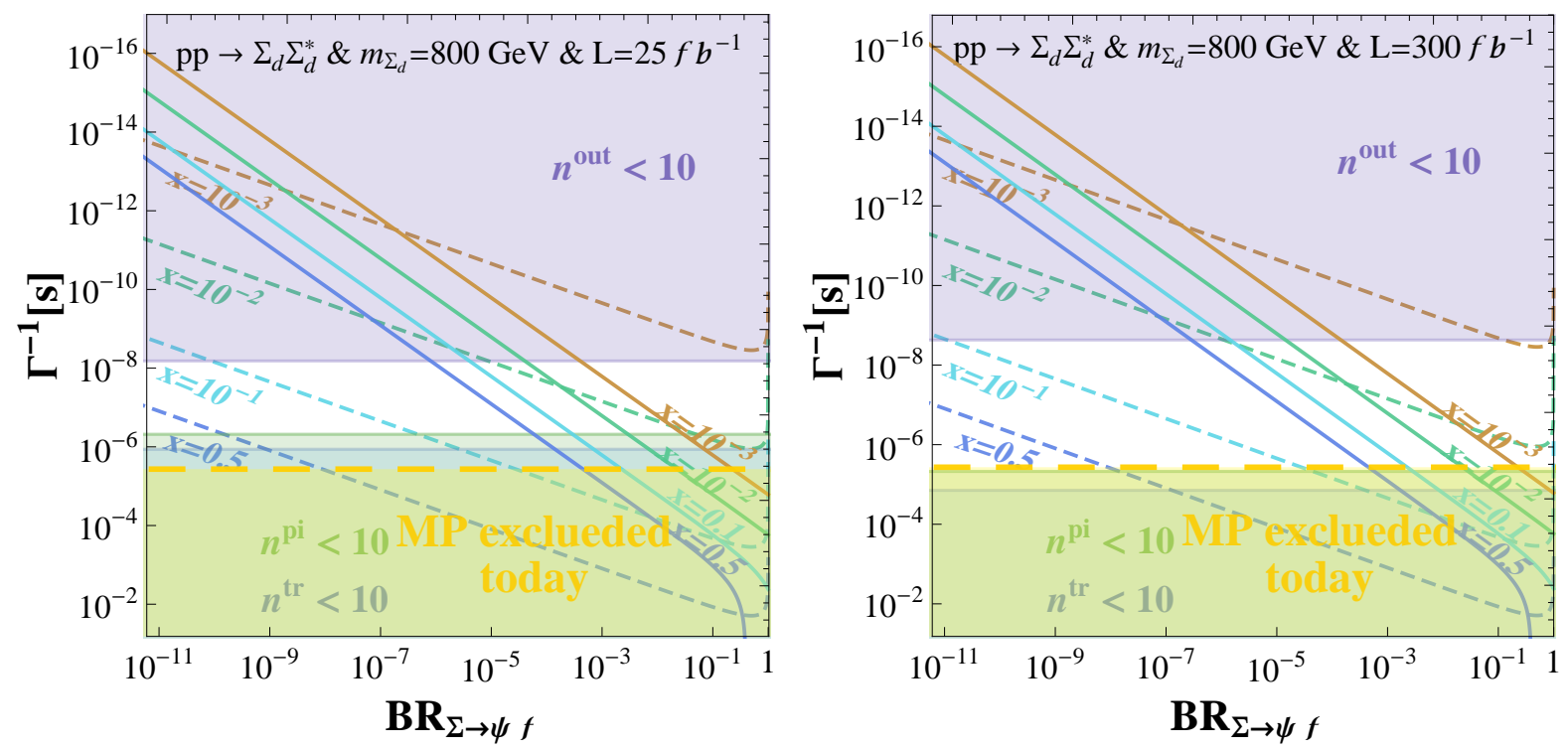

FiguRE 4.4: LHC detection reach compared with the constraints from DM phenomenology in the plane $\left(\operatorname{Br}\left(\Sigma_{d} \rightarrow D M\right), \Gamma_{\Sigma_{d}}^{-1}\right)$. The shaded green (magenta) region corresponds to more than 10 decay events happening in the pixel or tracker (outside the detector). The double detection region is the white strip comprised between the shaded regions. We have fixed $m_{\Sigma_{d}}=800 \mathrm{GeV}$ and $\mathcal{L}=25 \mathrm{fb}^{-1}$ (left plot) and $\mathcal{L}=300 \mathrm{fb}^{-1}$ (right plot). The yellow shaded region, below the thick long-dashed yellow line, is excluded by current bounds on metastable particles.

In order to investigate this possibility we have performed a more focused study on a benchmark set of parameters, namely $m_{\Sigma_{d}}=800 \mathrm{GeV}$, corresponding to the maximal production cross-section, $m_{\psi}=10 \mathrm{GeV}$ and $\lambda$ and $\lambda^{\prime}$ fixed to reproduce the correct DM relic density and a DM lifetime of approximately $10^{28}$ seconds. We have reported on Table 4.1 the number of decay events, expected at the three values of the luminosity, namely 25, 300 and $3000 \mathrm{fb}^{-1}$, together with an estimate of the number of events in the two different type of decay channels, namely in DM plus SM fermion and two SM fermions. As evident from this Table, no events of decay into DM can be observed inside the detector (namely pixel or tracker), except possibly in a high luminosity run of LHC. This is a consequence of the very suppressed branching ratio of decay of $\Sigma_{d}$ into $\mathrm{DM}, \approx 10^{-3}$, required to reconcile the correct DM relic density with observable decays of the latter. A potential observation of decay of $\Sigma_{d}$ inside the detector seems thus limited to the pure SM channel. Possible dedicated searches should be then optimized for the search of displaced vertices with multi-jet/leptons and low amounts of missing energy.

The observation of the DM channel might be feasible because of the possibility of observing decays of stopped particles in the detector. For this reason, we have until now assumed that the decays of $\Sigma_{d}$ can be observed in the pixel/tracker region while beyond these regions only escaping tracks associated to the particle $\Sigma_{d}$ itself are detected. In reality colored/electromagnetically charged metastable particles lose energy by interacting with the detector material and a fraction of them can be stopped inside the detector itself. Their late time decays can be observed in the intervals between the collisions of proton beams [120,122]. These kinds of searches are characterized by rather low efficiencies, in particular because of the low, namely $\lesssim 10 \%$, fraction of stopped particles; as a consequence one should focus on the regions in which the number of decays outside the pixel/tracker regions is maximal, possibly renouncing to a statistically 

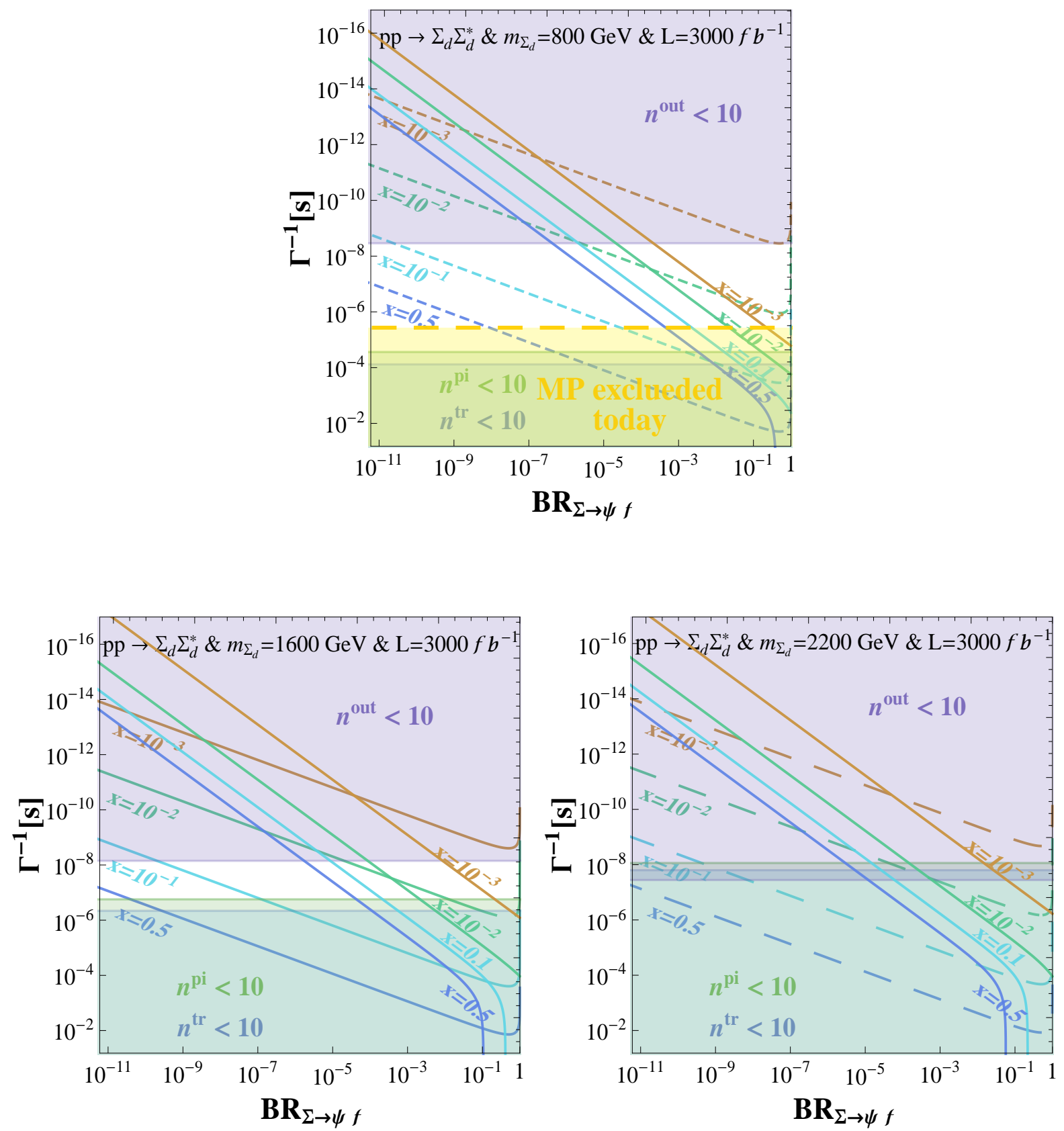

Figure 4.5: The same as Figure (4.4) but with $m_{\Sigma_{d}}=800 \mathrm{GeV}$ (upper plot), $m_{\Sigma_{d}}=1600 \mathrm{GeV}$ (left plot) and $m_{\Sigma_{d}}=2200 \mathrm{GeV}$ (right plot) and $\mathcal{L}=3000 \mathrm{fb}^{-1}$ in all cases.

significant number of events inside the detector. So searches for stopped particles are in some sense complementary to the searches discussed in this work. At the same time, as one can see in the regions where the number of particles decaying outside the detector is maximal (e.g. see Figure 4.4 and 4.5), the isolines of DM lifetime and relic density cross at values of the branching ratios of order 0.5 and thus a contemporary detection of both decays of $\Sigma_{d}$ is feasible provided the presence of statistically relevant population of stopped particles.

This possibility is confirmed by the outcome of the analysis reported in Table 4.2, where a benchmark with $\left(m_{\psi}, m_{\Sigma_{d}}\right)=(500,1000) \mathrm{GeV}$ has been considered and the couplings set in order to achieve, besides the correct DM relic density, a lifetime of the DM of $10^{28} \mathrm{~s}$ and a branching 


\begin{tabular}{|c|c|c|c|}
\hline Part of detector & Total & $\Sigma \rightarrow D M$ & $\Sigma \rightarrow$ SM only \\
\hline $\mathcal{L}=25 \mathrm{fb}^{-1}$ & \multicolumn{3}{|l|}{} \\
\hline pixel & 0 & 0 & 0 \\
\hline tracker & 0 & 0 & 0 \\
\hline out & 355 & 37 & 318 \\
\hline $\mathcal{L}=300 \mathrm{fb}^{-1}$ & \multicolumn{3}{|l}{} \\
\hline pixel & 0 & 0 & 0 \\
\hline tracker $^{\mid}$ & 0 & 0 & 0 \\
\hline out $^{-1}$ & 4267 & 445 & 3822 \\
\hline $\mathcal{L}=3000 \mathrm{fb}^{-1}$ & \multicolumn{3}{|l}{} \\
\hline pixel & 0 & 0 & 0 \\
\hline tracker & 0 & 0 & 0 \\
\hline out & 42670 & 4450 & 38220 \\
\hline
\end{tabular}

TABLE 4.2: The same as Table 4.1 but for $m_{\Sigma_{d}}=1 \mathrm{TeV}, x=0.5$ and the couplings $\lambda$ and $\lambda^{\prime}$ set to, respectively, $1.2 \times 10^{-12}$ and $3.6 \times 10^{-12}$, in order to achieve the correct DM relic density and a DM lifetime of $10^{28} \mathrm{~s}$.

\begin{tabular}{|c|c|c|c|}
\hline Part of detector & Total & $\Sigma \rightarrow D M$ & $\Sigma \rightarrow$ SM only \\
\hline $\mathcal{L}=25 \mathrm{fb}^{-1}$ & \multicolumn{3}{|l|}{} \\
\hline pixel & 97 & 15 & 82 \\
\hline tracker & 115 & 18 & 97 \\
\hline out $^{\mid}$ & 4 & 0 & 4 \\
\hline $\mathcal{L}=300 \mathrm{fb}^{-1}$ & \multicolumn{3}{|l}{} \\
\hline pixel & 1169 & 184 & 985 \\
\hline tracker $^{\mid} 1384$ & 218 & 1166 \\
\hline out $^{-1}$ & 50 & 8 & 42 \\
\hline $\mathcal{L}=3000 \mathrm{fb}^{-1}$ & \multicolumn{3}{|l}{} \\
\hline pixel & 11695 & 1849 & 9846 \\
\hline tracker & 13846 & 2189 & 11657 \\
\hline out & 499 & 79 & 420 \\
\hline
\end{tabular}

TABLE 4.3: The same as Table 4.1 but for $m_{\Sigma_{d}}=1 \mathrm{TeV}, x=10^{-6}$ and the couplings $\lambda$ and $\lambda^{\prime}$ set to, respectively, $6.5 \times 10^{-10}$ and $1.5 \times 10^{-9}$. For this choice of parameters $\operatorname{Br}\left(\Sigma_{d} \rightarrow D M\right) \sim 0.15$ and the DM relic density is entirely achieved through the freeze-in mechanism. The DM lifetime instead exceeds of many orders of magnitude the sensitivity of present and next future detectors.

ratio of decay of $\Sigma$ into DM of approximately $10 \%$. For luminosities above $300 \mathrm{fb}^{-1}$, a sizable number of events could be observed in both the decay channels of $\Sigma_{d}$, possibly compensating the low efficiency in the detection of stopped particles. In order to explore quantitatively this scenario an analysis accounting for the typology of decay products of $\Sigma_{d}$ as well as a simulation of the detector are however needed. This is beyond the scope of this work and will be left to a future study.

The contemporary detection of both decay channels of $\Sigma_{d}$ can be feasible as well at very low values of $x$, thus corresponding to very light DM particles. Indeed in such a case it is possible to set the two couplings $\lambda$ and $\lambda^{\prime}$ to comparable values without conflicting with ID constraints because of the strong enhancement, namely $\propto x^{-5}$ of the DM lifetime. We also notice that higher values of the coupling $\lambda$ are favored by the DM relic density since it tends 


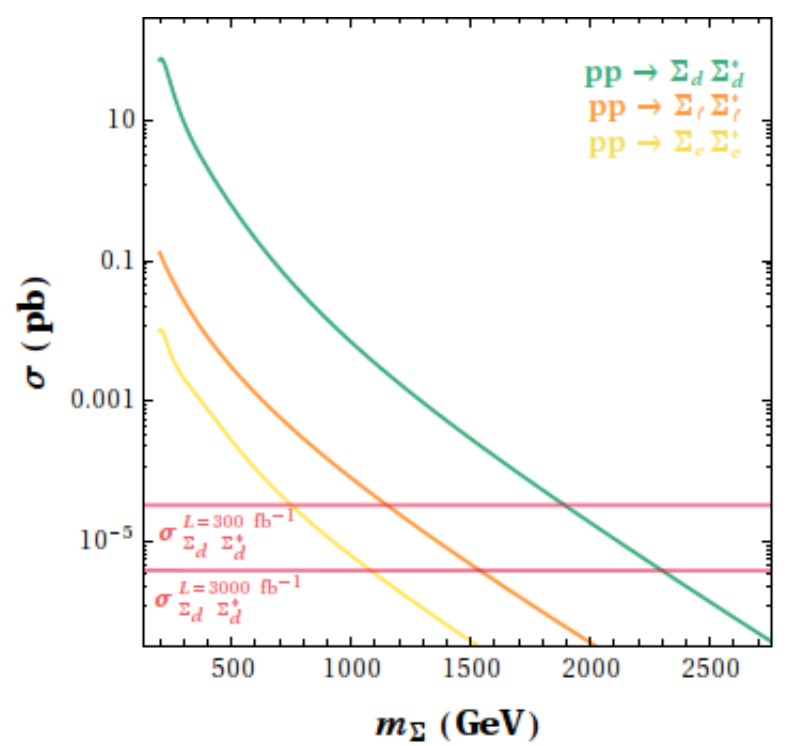

FiguRE 4.6: $N L O$ cross-section for several kinds of $\Sigma_{f}$ field, namely $\Sigma_{d}, \Sigma_{\ell}$ and $\Sigma_{e}$. We report as well the minimal value of the production cross-section, for two values of the luminosity, namely 300 and $3000 \mathrm{fb}^{-1}$, needed to give 5 pairs of particles.

to be suppressed as $x$. In such scenario we thus expect $\Sigma_{f}$ particles to be relatively short-lived and thus most of the decay events happen in the pixel/tracker region. This is confirmed by the results shown in Table 4.3 in which we have considered a benchmark model with the very low value $x=10^{-6}$. As we expected, most of the decay events lie in the inner detector with statistically relevant populations in both the decay channels. For luminosities above $300 \mathrm{fb}^{-1}$ it is nonetheless possible to observe more than 10 escaping tracks; the LHC thus provides an optimal reconstruction of the properties of the scalar field. On the other hand for the very low value of $x$ considered the Indirect Detection of DM is not possible since its lifetime largely exceeds present and next future experimental sensitivity. As a consequence, in this kind of setup, LHC is the only probe of the model under consideration.

\subsubsection{EW-charged scalar}

In this section we present a similar analysis for $\Sigma_{e, \ell}$-type scalar field. First of all we assume, analogously to the previous scenario, that the two components of the $S U(2)_{L}$ doublet are exactly degenerate in mass, such that the analysis can be carried out with the exact same steps as before. The case in which, instead, a sizable mass splitting is present is also phenomenologically intriguing. In fact, since one of the components of $\Sigma_{\ell}$ is electrically neutral, it remains undetectable at LHC in case of decays outside the pixel/tracker region, and thus it behaves like an additional DM component. It is also worth noticing that additional interesting collider signatures can arise from the case in which both the components are accessible to LHC production and decays in $\mathrm{W}$ boson (either on- or off-shell) are allowed. We are going to comment on this possibility at the end of this subsection.

We show in Figure 4.6 the production cross-section of the $\Sigma_{\ell}$ and $\Sigma_{e}$ type fields, compared with the one of $\Sigma_{d}$. As we can see the production cross-sections are sensitively lower, with respect to the colored case, with a maximal mass reach, corresponding to the high luminosity upgrade of LHC, of approximately $1400 \mathrm{GeV}$. 
Figure 4.7 and 4.8 show the LHC reach, in the three detection regions, as function of the couplings $\lambda$ and $\lambda^{\prime}$ and for some fixed values of $x$, for two values of $m_{\Sigma_{\ell, e}}$, namely 400 and $800 \mathrm{GeV}$. In the lower mass scenario it is again present an upper bound on the lifetime of the scalar field coming from the current searches of disappearing tracks. Notice that this last limit is stronger in the case of $\Sigma_{\ell}$ type field as consequence of the higher cross-section at a given value of the mass. At the same time, the limits arising from the detector stable particles are substantially relaxed with respect to the case of colored particles so that we can consider values of the mass as low as $300-400 \mathrm{GeV}$. We also notice that the production cross-section of $\Sigma_{\ell}$ pairs is sensitively larger with respect to the case of a $S U(2)_{L}$ singlet. This enhancement is substantially due to the process $p p \rightarrow \Sigma_{\ell}^{ \pm} \Sigma_{\ell}^{0}$, with $\Sigma_{\ell}^{ \pm}$and $\Sigma_{\ell}^{0}$ being, respectively, the electrically charged and neutral components of the $S U(2)$ doublet. On the other hand the increase in the expected number of events depends on the lifetime of $\Sigma_{\ell}$. It is indeed maximal at shorter lifetimes, when the scalar field decays prevalently in the inner detector, since displaced vertices can be detected both for electrically charged and neutral mother states, while it is more moderate at the longest lifetimes since only the charged component of the $S U(2)_{L}$ doublet can manifest as metastable tracks while the neutral one escapes detection. Contrary to the colored case it is possible to have sizable or even dominant contribution from the SuperWIMP mechanism at masses accessible to the LHC production. However, as evident from the plot, this occurs only at very small values of the couplings, such that the decays of the scalar field occur substantially only outside the detector.

In this case we have also reformulated our results in the plane $\left(\Gamma_{\Sigma}^{-1}(\mathrm{~s})-\mathrm{BR}\left(\Sigma_{f} \rightarrow \psi f\right)\right)$. As evident from Figure 4.9 and 4.10 the LHC "double" detection region defined in the previous sections is extremely narrow, as consequence of the lower number of expected events, due to the lower production cross-sections, and it is already closed, for masses of $800 \mathrm{GeV}$, in the case of $\Sigma_{e}$-type field, which thus features extremely poor detection prospects. We also notice that the crossing of curves of the relic density and the DM lifetime occurs, in the double detection strip, at values of the branching ratio at most of the order of $10^{-3}$ which again makes very difficult the detection of the decay channel of $\Sigma_{\ell, e}$ into DM.

\section{Benchmarks for EW $\Sigma_{f}$}

Analogously to the previous section, we have investigated the prospects of detection of $\Sigma_{\ell, e}$ from a more quantitative perspective focussing on a benchmark set of parameters. We have indeed considered the pair production of a $\Sigma_{\ell}$ particle with an assignment of $(10,400) \mathrm{GeV}$, corresponding to the maximal production cross-section, for $\left(m_{\psi}, m_{\Sigma_{\ell}}\right)$ and fixed the couplings to give the correct relic density and a lifetime compatible with the current experimental limits. As it is evident from the results which are reported in Table 4.4, the number of expected events is reduced with respect to the colored scalar case. A statistically relevant number of decay events both in the inner and outer part of the detector appears nevertheless already at low luminosity. Note that indeed the severe limit from the searches of metastable tracks (cfr. Figure 4.7) forces the choice of rather low lifetimes of $\Sigma_{\ell}$, such that most of the events concentrate in the inner detector. The bound on the lifetime is less severe for a $\Sigma_{e}$-type field with the same mass. At the same time the total number of events is drastically reduced, because of the sensitively lower cross-section, and then a double LHC detection would require higher luminosities. We also remark that the choice of the pair $\left(\lambda, \lambda^{\prime}\right)$, made to comply with all experimental constraints, corresponds to an extremely suppressed branching ratio of decay into DM, such that no events with $\Sigma_{\ell}$ decaying into DM are observed, even considering a high luminosity upgrade of the 

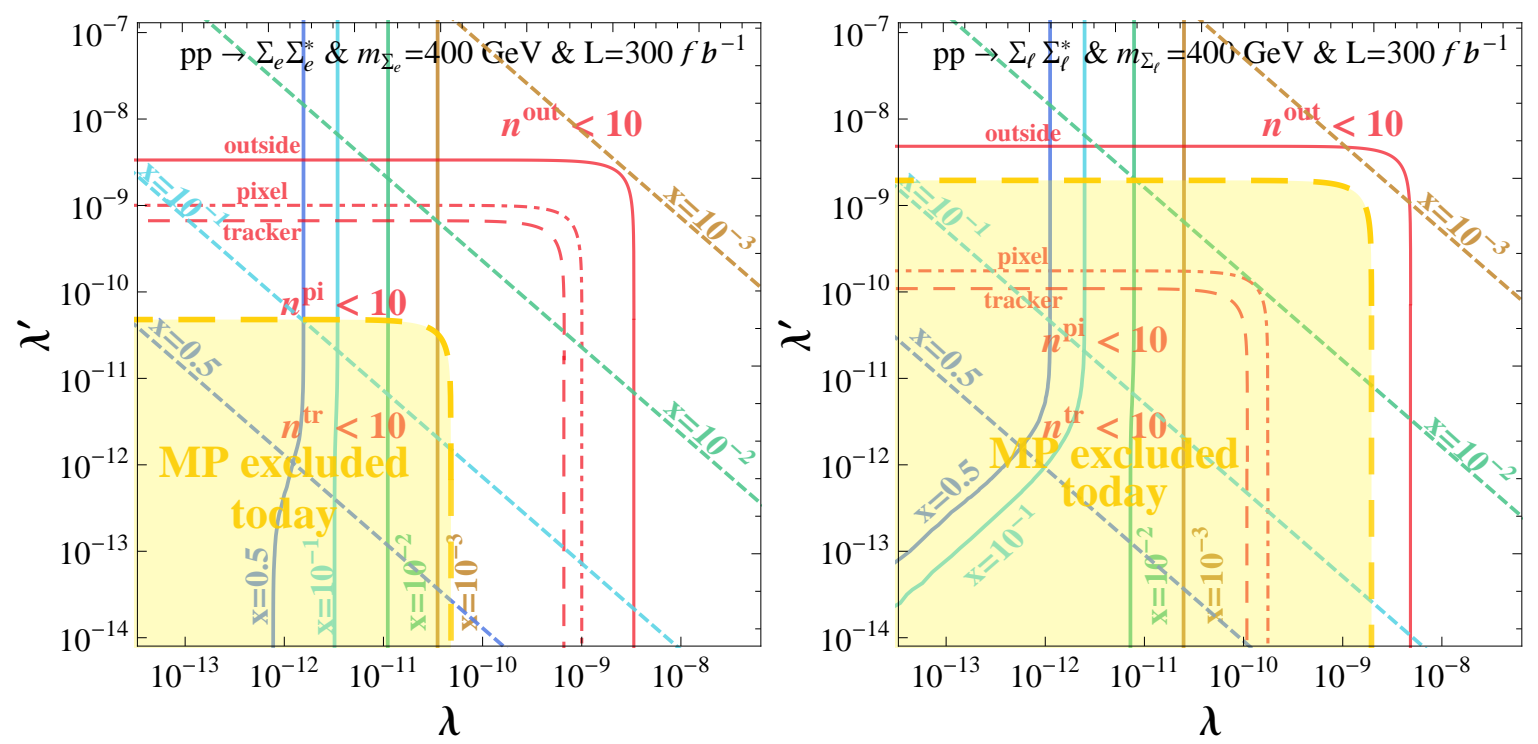

FIGURE 4.7: LHC reach, in the plane $\left(\lambda, \lambda^{\prime}\right)$, in the pixel (red dot-dashed lines), tracker (red dashed lines) and outside the detector (red solid lines), for an integrated luminosity of $300 \mathrm{fb}^{-1}$ for a $\Sigma_{e}$ (left panel) and a $\Sigma_{\ell}$ (right panel) scalar field of mass $400 \mathrm{GeV}$. The solid lines represent the cosmological value of the DM relic density for $x=\left\{10^{-3}, 10^{-2}, 0.1,0.5\right\}$, while the shortdashed lines represent a reference value of $10^{28} \mathrm{~s}$, approximately corresponding to the current experimental sensitivity on the DM lifetime for the considered set of values of $x$. The yellow region below the thick long-dashed yellow line is excluded by searches of metastable particles.
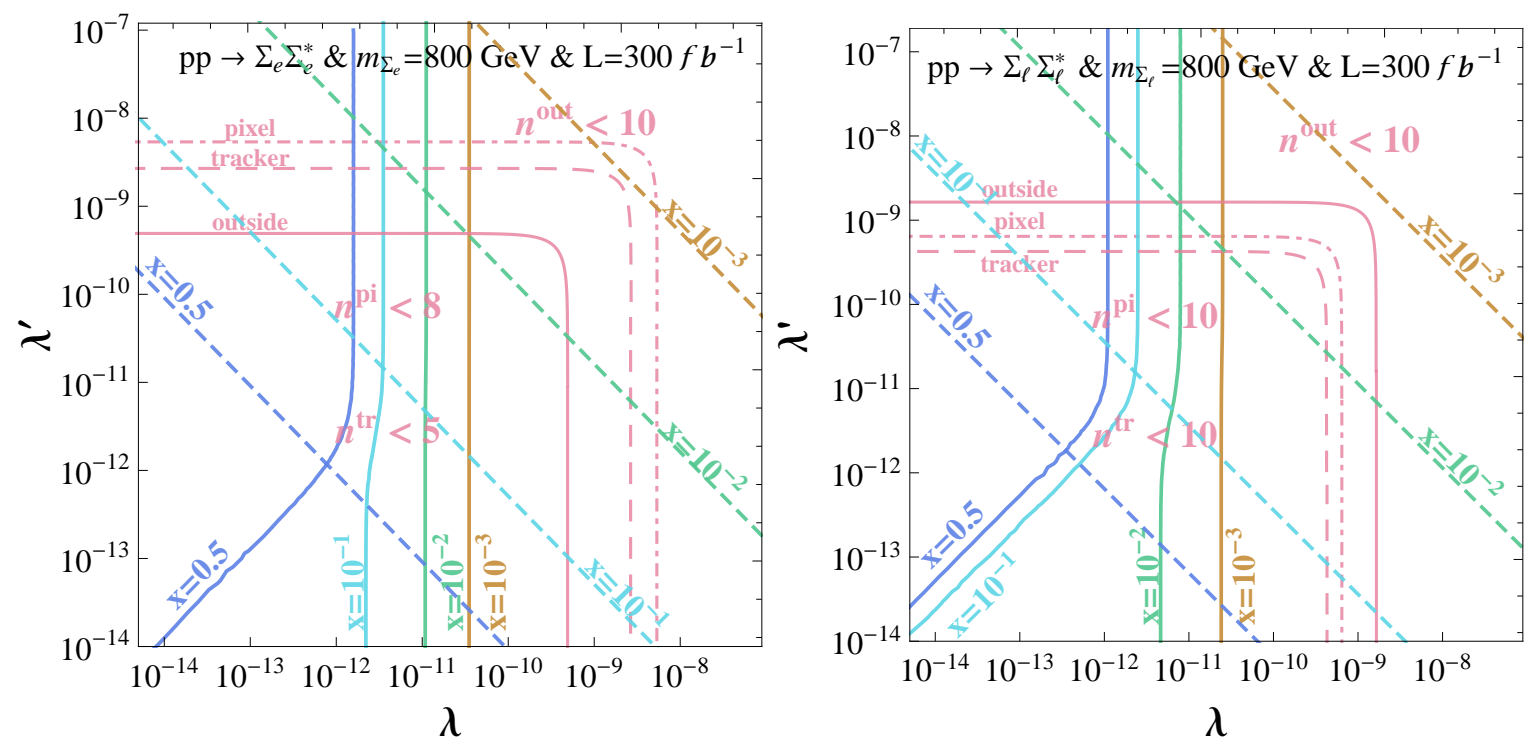

FIGURE 4.8: The same as Figure 4.7 but for $m_{\Sigma_{e, \ell}}=800 \mathrm{GeV}$.

LHC. We could in principle consider slightly higher value of the mass of $\Sigma_{\ell}$, which would allow to achieve $\mathrm{BR} \sim 10^{-3}$, since the limit from searches of metastable tracks promptly disappears, which would allow the observation of the DM channel in the high luminosity upgrade of LHC. However, a strong reduction of the total number of event would occur as well, because of the steep dependence of the production cross-section with $m_{\Sigma_{\ell}}$, such that this possibility is already 

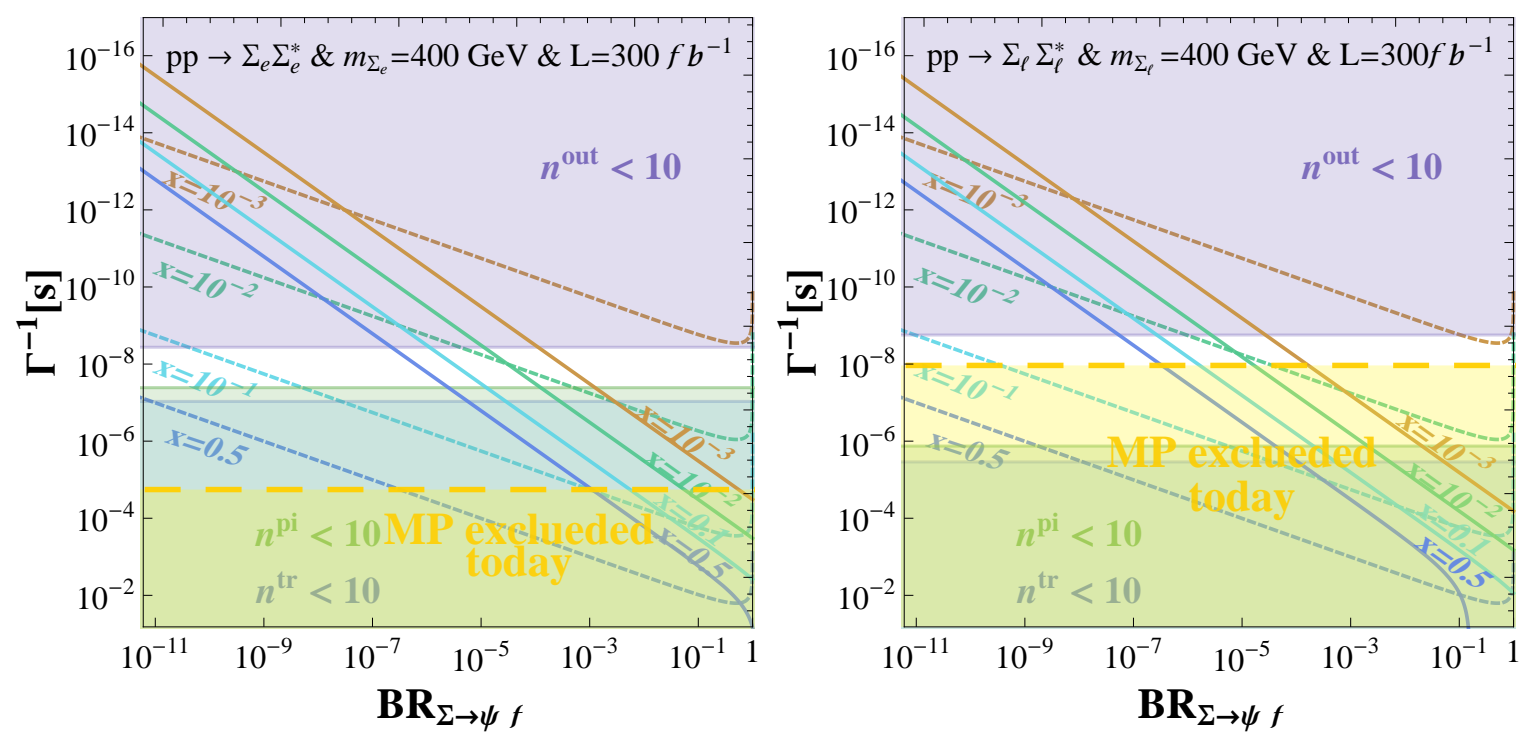

FiguRE 4.9: LHC reach for $\Sigma_{e}$ (left) and $\Sigma_{\ell}$ (right) in the plane $\left(B r\left(\Sigma_{e, \ell} \rightarrow D M\right), \Gamma_{\Sigma}^{-1}\right)$ for integrated luminosity of $300^{6}, f b^{-1}$ and $m_{\Sigma_{e, \ell}}=400 \mathrm{GeV}$. The violet, green and blue region represent the regions where less then 10 events are expected, respectively in the 'outside', tracker and pixel regions. The 'double detection' region, defined in the main text, is thus the white strip between the shaded regions. The solid and dashed lines giveagain the cosmological relic density and the reference DM lifetime for the set $x=\left\{10^{-3}, 10^{-2}, 0.1,0.5\right\}$. The yellow region below the thick dashed yellow line is excluded by current searches of metastable particles.
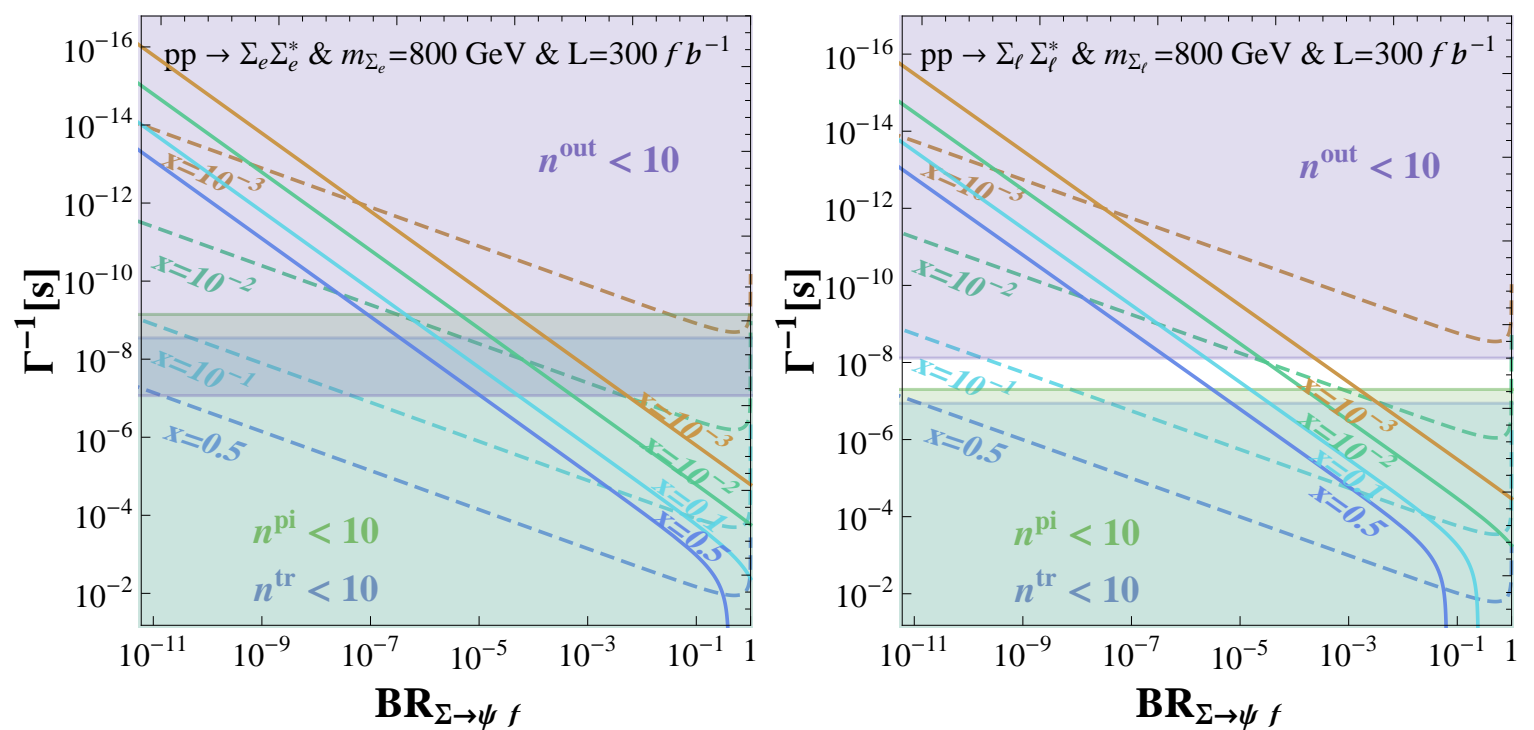

Figure 4.10: The same as Figure 4.9 but for $m_{\Sigma_{e, \ell}}=800 \mathrm{GeV}$. A 'double detection' region is present only in the case of $\Sigma_{\ell}$-type field.

excluded for masses around $500 \mathrm{GeV}$. We thus conclude that, substantially, it will not be possible to identify at LHC the peculiar feature of our model, namely the presence of two (i.e. DM+SM and SM only) decay channels.

Analogously to the colored scenario we can consider signals outside the double detection regions. In the high lifetime region only the decay channel into SM particles might be accessible to possible searches of stopped particles since the small number of events, consequence of the 


\begin{tabular}{|c|c|c|c|}
\hline Part of detector & Total & $\Sigma \rightarrow D M$ & $\Sigma \rightarrow$ SM only \\
\hline \multicolumn{4}{|l|}{$\mathcal{L}=25 \mathrm{fb}^{-1}$} \\
\hline pixel & 92 & 0 & 92 \\
\hline tracker & 156 & 0 & 156 \\
\hline out & 19 & 0 & 19 \\
\hline \multicolumn{4}{|l|}{$\mathcal{L}=300 \mathrm{fb}^{-1}$} \\
\hline pixel & 762 & 0 & 762 \\
\hline tracker & 1285 & 0 & 1285 \\
\hline out & 230 & 0 & 230 \\
\hline \multicolumn{4}{|l|}{$\mathcal{L}=3000 \mathrm{fb}^{-1}$} \\
\hline pixel & 7621 & 0 & 7621 \\
\hline tracker & 12855 & 0 & 12855 \\
\hline out & 2300 & 0 & 2300 \\
\hline
\end{tabular}

TABLE 4.4: Number of decay events, total as well as separately in the two kind of decay channels (DM or SM only), expected in the three detection regions at the LHC for the indicated values of luminosity. The benchmark chosen consist in a $\Sigma_{\ell}$-type field with mass of $400 \mathrm{GeV}$ while the $D M$ mass is set to $10 \mathrm{GeV}$. The pair $\left(\lambda, \lambda^{\prime}\right)$ has been fixed to $\left(5 \times 10^{-12}, 2.5 \times 10^{-9}\right)$. Notice that for the inner detector, i.e. pixel and tracker, we have counted the decays of both the charged and neutral components of the doublet while in the outside region only the charged component has been considered.

\begin{tabular}{|c|c|c|c|}
\hline Part of detector & Total & $\Sigma \rightarrow D M$ & $\Sigma \rightarrow$ SM only \\
\hline \multicolumn{4}{|l|}{$\mathcal{L}=25 \mathrm{fb}^{-1}$} \\
\hline pixel & 1 & 1 & 0 \\
\hline tracker & 2 & 1 & 1 \\
\hline out & 8 & 4 & 4 \\
\hline \multicolumn{4}{|l|}{$\mathcal{L}=300 \mathrm{fb}^{-1}$} \\
\hline pixel & 12 & 6 & 6 \\
\hline tracker & 27 & 14 & 13 \\
\hline out & 94 & 48 & 46 \\
\hline \multicolumn{4}{|l|}{$\mathcal{L}=3000 \mathrm{fb}^{-1}$} \\
\hline pixel & 120 & 62 & 58 \\
\hline tracker & 270 & 139 & 131 \\
\hline out & 941 & 484 & 457 \\
\hline
\end{tabular}

TABLE 4.5: Number of decay events, total as well as separately in the two kind of decay channels (DM or SM only), which is expected in the three detection regions at the LHC for the indicated values of luminosity. The benchmark chosen consist in a $\Sigma_{\ell}$-type field with mass of $800 \mathrm{GeV}$ while the DM mass is set to $8 \times 10^{-4} \mathrm{GeV}$. The pair $\left(\lambda, \lambda^{\prime}\right)$ has been fixed to $\left(8 \times 10^{-10}, 7.8 \times\right.$ $10^{-10}$ ) and corresponds to a lifetime many orders of magnitude above current and next future experimental sensitivity. No ID detection is thus expected in this case. Analogously to tab. (4.4), for the inner detector, i.e. pixel and tracker, we have counted the decays of both the charged and neutral components of the doublet while in the outside region only the charged component has been considered.

low production cross section, does not allow to have a statistically relevant number of events in both the decay channels ${ }^{3}$. Alternatively we can consider very low values of the DM mass in

\footnotetext{
${ }^{3}$ We also notice that there are not, at the moment, searches of only electroweakly interacting stopped particles. On general grounds we expect a lower detection efficiency with respect to the case of color interaction.
} 
order to evade ID constraints and for $x \sim 10^{-6}$ it might be again possible to observe both the $\Sigma_{\ell}$ decay channels, renouncing however to the possibility of next future detection of DM decays in cosmic rays. A possible outcome in this kind of scenario is reported in Table 4.5, where it is shown the number of expected observed decay events in the case of $\Sigma_{\ell^{-}}$type field with mass of $800 \mathrm{GeV}$ and for a very low value of the DM mass corresponding to $x=10^{-6}$. The value of the $\left(\lambda, \lambda^{\prime}\right)$ pair, respectively $8 \times 10^{-10}, 7.8 \times 10^{-10}$, guarantees the correct DM relic density, through the freeze-in mechanism, and a branching fraction of decay of the scalar field into DM of approximately $50 \%$. Although the choice of the parameters is substantially analogous to the low mass benchmark studied in the case of color charged scalar (the lifetimes of the scalar field differ approximately by a factor 2) the majority of the events now lie in the "outside" region. This is due to the fact the $\Sigma_{\ell, e}$ are more boosted with respect to colored scalars. At the higher luminosities we have anyway a number of events in the inner detector, for both decay channels, satisfying our discovery requirement. We have thus again a good capability for LHC of providing information on the model under consideration, renouncing however at any prospects of indirect detection of the decays of the DM.

We conclude this section describing how the LHC signals may result altered, in the $\Sigma_{\ell}$ scenario, in the presence of a sizable mass splitting in the EW multiplet. As already mentioned in this case the heaviest component of the doublet dominantly decays into the lighter one and a $\mathrm{W}$ boson (or into two quarks/leptons) and these decays result prompt for mass-splitting above $\sim 1 \mathrm{GeV}$. Contrary to the colored case, we can have a sizable production of the heavy component through the process $p p \rightarrow \Sigma_{l}^{ \pm} \Sigma_{l}^{0}$, being mediated by the $W$ boson, which can account for $\sim 50 \%$ of the total production cross-section even for sizable mass splittings. The range of signals results therefore enriched if the detection of the products of the prompt decays of the heavy component of the doublet is possible.

In the case $m_{\Sigma^{ \pm}}>m_{\Sigma_{0}}$ the decays $\Sigma^{ \pm} \rightarrow \Sigma^{0} f \bar{f}^{\prime}$ produce displaced vertices accompanied by prompt leptons and jets, for short lifetimes of $\Sigma^{0}$, or a signal consisting of jet/leptons + missing energy, customarily studied in supersymmetric setups $[173,174]$, in the case that $\Sigma^{0}$ decays outside the inner detector ${ }^{4}$. In the opposite case $m_{\Sigma^{ \pm}}<m_{\Sigma^{0}}$ prompt jets/leptons are associated to displaced vertices or metastable tracks.

\section{A glimpse of Linear Colliders}

In the light of the discovery of the Higgs boson, a very strong impetus to study the properties of this new particle has been given to the particle physics. Proton machines are not suitable for this, due to the large undetermination of the collision of quarks and gluons. A linear lepton collider would offer a cleaner environment.

The International Linear Collider (ILC) is a proposal of a new accelerator which would collide electron with positron along a straight path. It is planned to have a collision energy of $500 \mathrm{GeV}$ initially, with the possibility for a later upgrade to $1 \mathrm{TeV}$, and be between $30 \mathrm{~km}$ and $50 \mathrm{~km}$ long. In addition, this machine is supposed to operate at (at least) three stages. After a start at a center of mass energy of $250 \mathrm{GeV}$, there will be an increase of the center of mass energy to $500 \mathrm{GeV}$, and later to $1000 \mathrm{GeV}$. In these three stages, each one operating for three years, an integrated luminosity of 250,500 , and $1000 \mathrm{fb}^{-1}$ is, respectively, expected.

\footnotetext{
${ }^{4}$ Notice that we could as well consider monojet + missing energy signals in the case of long lived pair produced $\Sigma_{l}^{0}$, totally analogous to the DM pair production scenarios. However in our setup pair production of $\Sigma_{l}^{0}$ occurs only through a strongly off-shell Z-boson giving a rather weak signal $[175,176]$.
} 
The major advantage of colliding electrons and positrons compared to colliding hadrons (e.g. LHC) is that these particles are fundamental particles. This means that the initial state quantum numbers and energies of these point-like particles are known. Moreover, the final state of a collision between fundamental particles contains much less particles and therefore it is easier to reconstruct and analyse. Measurements can be thus done with a much higher precision. Since no QCD cross-section calculations are here involved, smaller theoretical uncertainties are also introduced. At ILC the precision measurements only rely on electroweak interactions with radiative corrections at the few percent level [177]. The straight path along which the two beams are accelerated gives another important advantage. This is the possibility for the incoming particle not to emit electromagnetic radiation (known as synchrotron radiation), thus saving energy to use it in producing heavier states. Finally, the use of polarised particles beams allows to enrich signal samples and reduce the contribution from background processes.

ILC covers many different measurements which are discussed in detail in [177]. However, the central topic of the physics program of the ILC will be the Higgs boson. The new machine will indeed be able to measure its mass, its lifetime, its production cross-sections, its branching ratios to quarks, leptons and bosons and, finally, the self-coupling with unprecedented precision.

The difference between hadron - hadron and $e^{+}-e^{-}$collisions has relevant implications for the detectors as well as the experimentation. The LHC detectors must consist of radiation-hard materials to deal with a high occupancy rate. They must have thick calorimeters to contain particles with a wide range of energies, requiring also the placement of solenoids inside the calorimeter volume. Moreover, complex trigger systems must be used to reduce the background and focus on the most interesting events. At ILC, instead, tracking detectors can be made as thin as technically feasible and, in addition, all elements, from the vertex detector to the calorimeter, can be brought much closer to the interaction point.

The Compact Linear Collider (CLIC) [178] is a proposal of another linear $e^{+}-e^{-}$collider that is planned to be the naturally evolution of ILC, namely a high-collision-energy and highluminosity ILC. It aims at accelerating and colliding electrons and positrons at a center of mass energy of $3 \mathrm{TeV}$. The main differences between CLIC and ILC are principally due to the fact that at CLIC the operating energy is higher and the bunch spacing is shorter (0.5 nanoseconds).

Two detector concepts/projects are foreseen for the future linear colliders ILC and CLIC: the International Large Detector (ILD) and the Silicon Detector (SiD). Since the basic layout of both detectors is very similar to one another, here we only briefly discuss the ILD. ILD is a multi-purpose detector, which consists of a tracking system and a calorimeter system. These two systems are located inside a solenoid, which provides a magnetic field of 3.5 Tesla. The tracking system consists of the Vertex Tracker (VTX) and the Time Projection Chamber (TPC). VTX is the closest tracker to the interaction point which aims at identifying heavy quarks (charm and bottom) and tau leptons by reconstructing corresponding decay vertexes. It is formed by three layers of double-sided ladders located in the barrel region of the detector. Moving outward, we find the TPC (central component of the tracker) for quasi-continuous track reconstruction, followed by a Silicon External Tracker(SET) consisting of inner and outer barrel silicon strip layers for precise track reference and a forward silicon strip tracker.

Below we are interested in performing the same collider analysis for $\Sigma_{\ell}$-type scalar field in view of CLIC rather than ILC because the latter runs at too low collision energies, and so it can not produce the heavy scalar particles in the final state we are looking for. The goal of this analysis is to understand if our prospects can improve by employing CLIC instead of LHC. As regards the choice of $S U(2)_{L}$ scalar doublet $\Sigma_{\ell}$, we point out that the colored scalar 

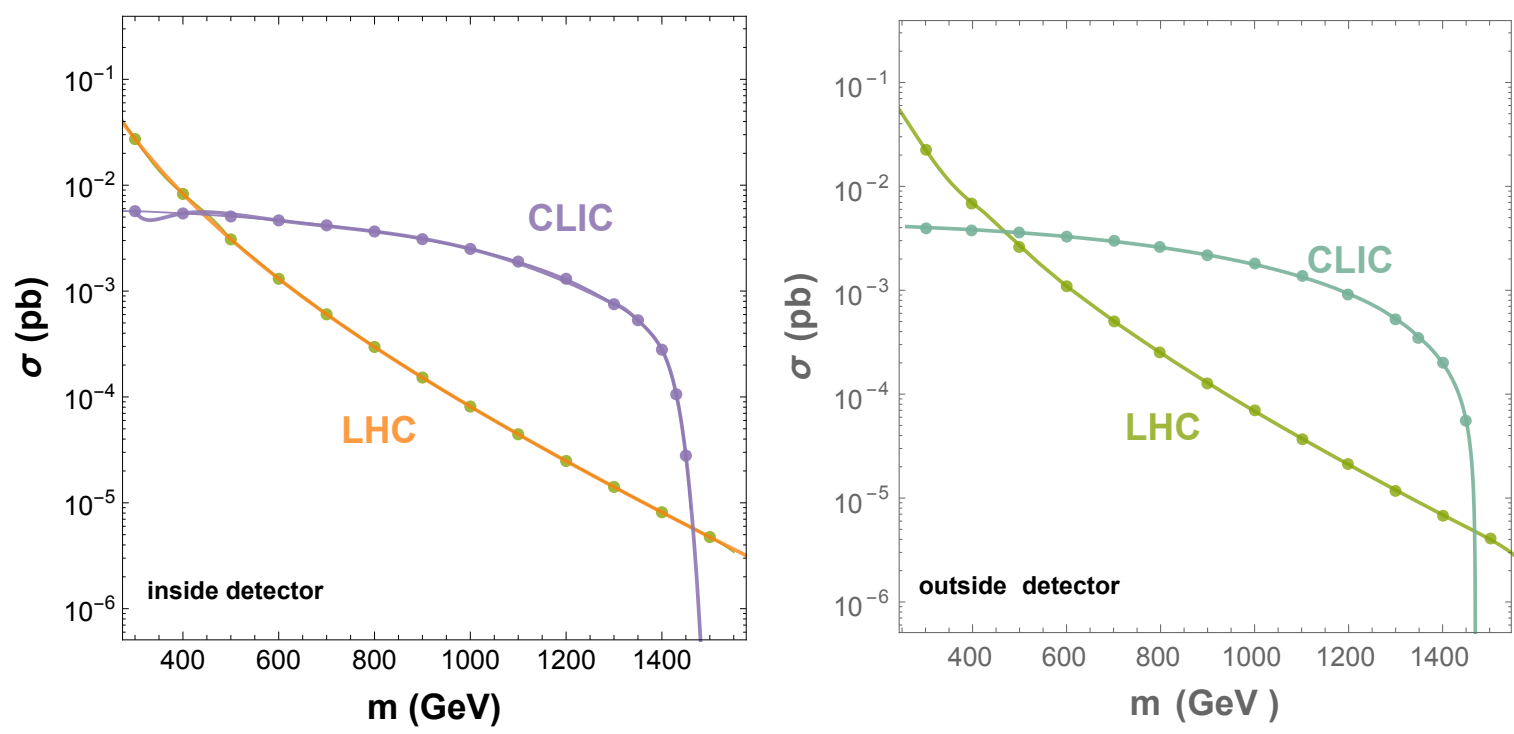

Figure 4.11: Left panel: Cross section of $\Sigma_{\ell}$-displaced vertices at LHC (orange curve) and CLIC (violet curve). Right panel: Cross section of $\Sigma_{\ell}$-metastable particles at LHC (pea green curve) and CLIC (green curve).

$\Sigma_{d}$ is not expected to make our prospects change substantially since the production channels at ILC always involve electroweak processes and therefore the $\Sigma_{d}$ production cross-section is comparable with $\Sigma_{\ell}$ one. Moreover, the colored field features even poorer detection prospects than the electroweak field because of the current LHC bounds which move its mass to bigger masses. Note that we have again used MadGraph 5 to perform such an extension of our analysis.

In Figure 4.11 the comparison of the cross-sections of $\Sigma_{\ell}$-production coming from the simulation of CLIC and LHC through MadGraph 5 is showed. In particular, on the left panel we see the results for displaced vertices (orange (violet) line at LHC (CLIC)) while on the right one the results for metastable particles (light green (light blue) line at LHC (CLIC)). By comparing these two plots, we can easily notice the different trend in $\Sigma_{\ell}$-production of CLIC and LHC as a function of the mass of the outgoing particle. The much lower LHC production cross-section for heavy particles than for light ones can be explained by the steeply falling parton distributions of the proton. Note that although the center of mass energy of CLIC is much smaller that the one of LHC, due to the above-mentioned features of the linear collider, eventually the CLIC production is better than the LHC one.

To the end of the next subsection we leave the CLIC reach for the direct production of $\Sigma_{\ell}$ at $L=300 \mathrm{fb}^{-1}$ as a function of the scalar mass and the coupling $\lambda^{\prime}$ in order to better compare the capabilities of LHC and CLIC.

\subsection{Discussion of results and conclusions}

In this section we summarize the outcome of the previous analysis and discuss in greater detail whether a hypothetical next future LHC signal can unambiguously discriminate the underlying particle physics scenario.

We show in Figure 4.12 and 4.13 the LHC reach, in the two scenarios of $\Sigma_{d^{-}}$and $\Sigma_{\ell^{-}}$-type field, as function of the mass of the scalar field and of the coupling $\lambda^{\prime}$, for the two assignments of the DM mass of 10 and $100 \mathrm{GeV}$. The luminosity has been set to $300 \mathrm{fb}^{-1}$. The coupling $\lambda$ 
has been determined as function of the other parameters, using Equations (1.86), (1.87), (1.88) and (2.49), according to the requirement of the correct DM relic density.

The red, blue and green lines in the plots correspond to the observation of 10 events respectively in the Tracker, Pixel and outside the detector region. The green region comprised between these lines is the "double detection" region, in which the observation of at least 10 events in the inner detector and at least 10 escaping tracks can be obtained. Above this region only displaced vertices can be observed at a statistically relevant amount while below metastable tracks are the only visible signal. The yellow regions in the plots are already excluded, for the considered values of the DM mass, by constraints from DM indirect detection ${ }^{5}$. As evident the possibility of "double" LHC detection is already ruled out for DM masses above $100 \mathrm{GeV}$ in the case of colored scalar and for masses above $10 \mathrm{GeV}$ in the case of EW charged scalar. Nevertheless for the colored case, as can be seen in the left panel of Figure 4.12 as well as in the benchmark in Table 4.1, the future indirect detection region just below the present bound lies exactly in the double detection corner for a DM mass of $10 \mathrm{GeV}$ and $m_{\Sigma}<1500 \mathrm{GeV}$ and in that case possibly all the four parameters of the model could be within reach in the next future. The possibility of observing only displaced vertices is disfavored as well by ID for these values of the DM mass, with the exception of the highest value of the scalar field mass, at the boundaries of the LHC reach. The more severe exclusion in this last case is due to the fact that the production of lighter scalar particles is considered, in turn implying higher decay rates for the DM.

We can now investigate in more detail which kind of information can be inferred from a hypothetical future LHC signal. At high DM masses, namely above 100 (10) GeV in the colored (only electroweakly interacting) $\Sigma_{f}$ scenario, the only signal which can be expected are metastable tracks. In such case it will be possible to give a lower bound on the scalar field lifetime or perhaps to measure it, although maybe with less precision with respect to a double detection scenario, from the decay of stopped $\Sigma_{f}$. From this it may be possible to determine the sum $\lambda^{2}+\lambda^{\prime}$ while, in absence of observation of the decay processes, it is not possible to determine the single values of the two couplings, which could allow to infer the DM production mechanism. This task could be achieved in case of an Indirect Detection of dark matter decay which would provide, besides the value of the DM mass, information on a different combination of the two couplings, namely their product $\lambda \lambda^{\prime}$. The complete identification of the model could then be achieved by verifying that the DM relic density, computed with the reconstructed parameters, matches the cosmological value.

At intermediate DM mass scales the LHC "double" detection region is instead still viable. This scenario guarantees the optimal reconstruction of the lifetime of the scalar field, as well as its mass which can be inferred by the cross section and by the energy of the decay products. As already pointed out these informations alone do not allow to infer the DM relevant properties like the mass, which it is in any case not accessible at the LHC, and the production mechanism. Indeed the latter would require the knowledge of the single values of the couplings $\lambda$ and $\lambda^{\prime}$ while the lifetime of $\Sigma_{f}$ depends on the sum $\lambda^{2}+\lambda^{\prime 2}$. The two couplings could be singularly inferred in case it is possible to distinguish, through the decay products in the displaced vertices, the two decay channels of the scalar field. However our study based on the relative branching fractions of the two decay channels of the scalar fields, confirmed from a quantitative perspective by the study of the two benchmarks reported in Table 4.1 and 4.4, has shown that only the pure SM decay channel is accessible to LHC detection. In this case it is possible to infer, from

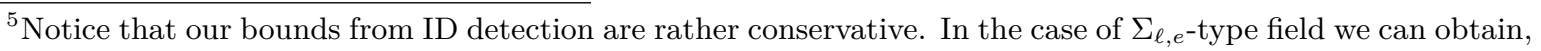
for example, weaker bounds assuming decays only in some flavour states like, e.g., $\tau$ leptons.
} 

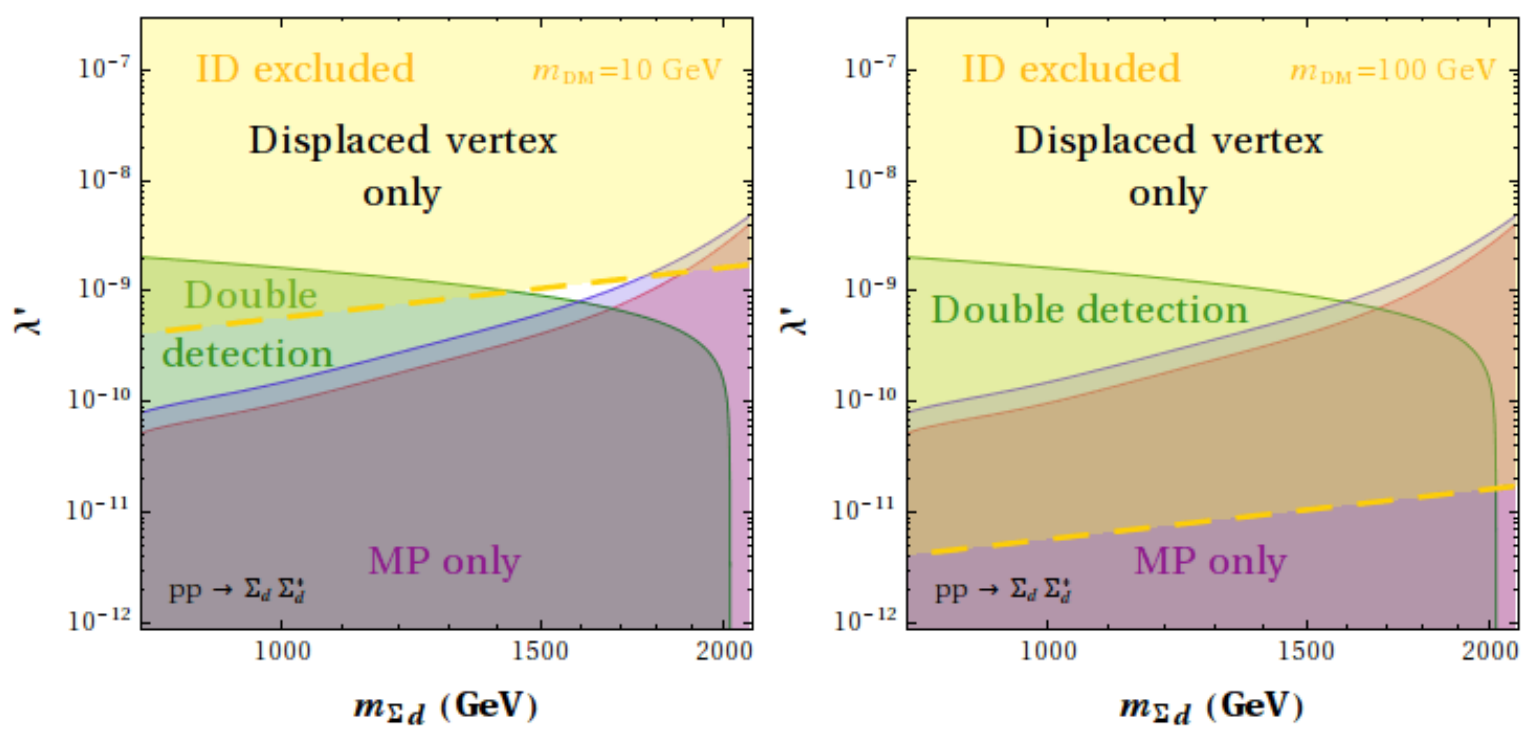

FiguRE 4.12: Summary of the possible observed signals at LHC, at $\mathcal{L}=300 \mathrm{fb}^{-1}$, for a $\Sigma_{d^{-}}$ type field, as function of its mass and the coupling $\lambda^{\prime}$. The other coupling, $\lambda$, has been fixed in order to reproduce the correct DM relic density while the DM mass has been set to $10 \mathrm{GeV}$ (left plot) and $100 \mathrm{GeV}$ (right plot). The red, blue and green lines correspond to the observation of 10 events respectively in the tracker, pixel and outside the detector region. In the "double" detection region, the green shaded region between these lines, a number $\geq 10$ of decay events in the pixel and the tracker and more than 10 tracks leave the detector. The yellow shaded region, above the thick yellow dashed line, is excluded by constraints from indirect detection of $D M$ decay.
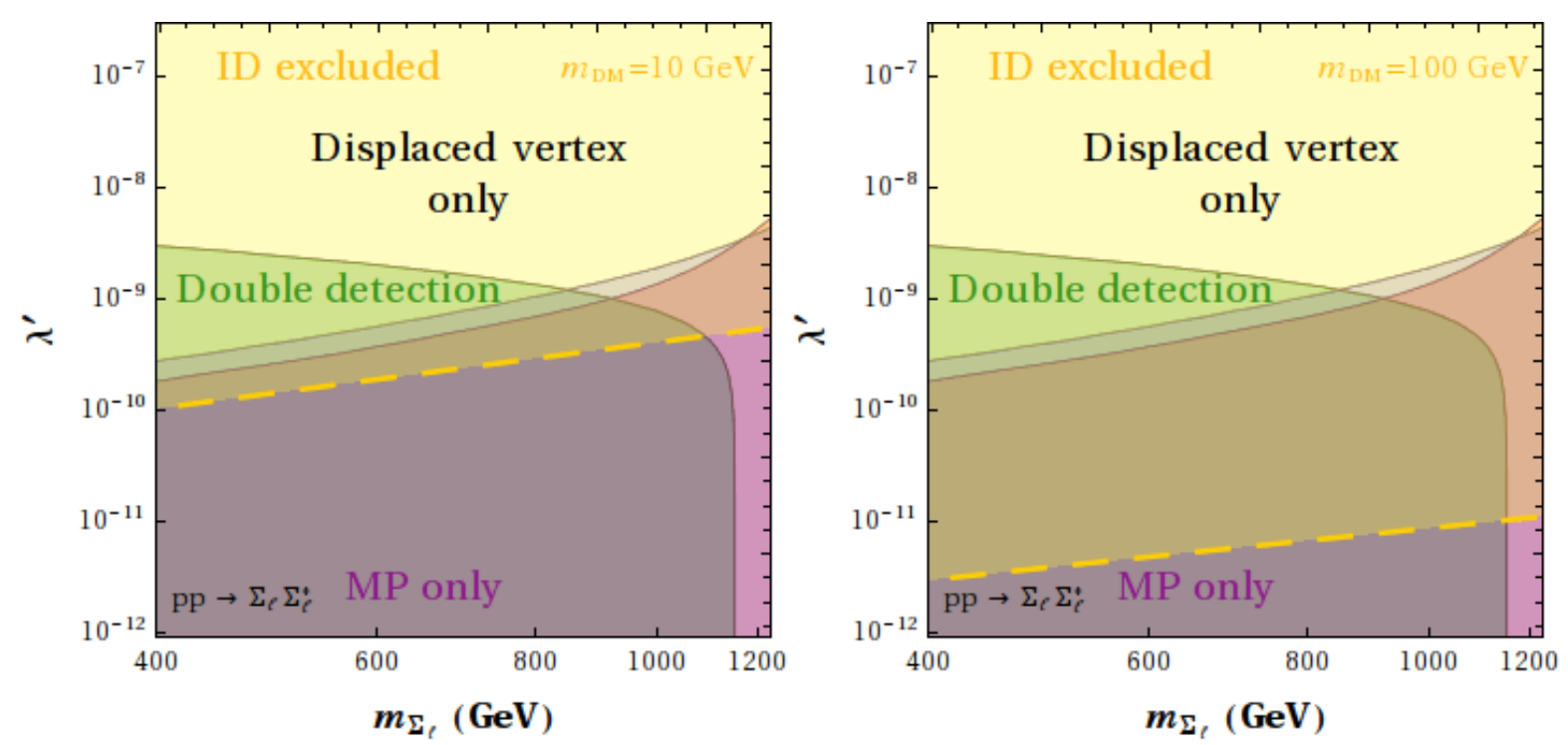

Figure 4.13: The same as Figure 4.12 but for the case of a $\Sigma_{\ell}$-type field.

the determination of the lifetime of the scalar field, only the value of $\lambda^{\prime}$ (assuming a negligible branching fraction of decay into DM). A LHC signal only can thus neither provide evidence of the existence of the DM nor information on its production mechanism. A correlation with a DM 
ID signal is again mandatory for determining the remaining parameters. In both the scenarios proposed above the capability of full determination of the model under consideration is hence limited to the regions of the parameters space which lie in proximity of the current experimental sensitivity to DM Indirect Detection.

For very low DM masses, LHC is instead the only probe of the model under consideration while ID is not achievable (as a consequence, bounds like the ones shown in Figure 4.12 and 4.13, are completely evaded). On the contrary the two branching fractions of decay into the scalar field, namely the one into DM+SM and only SM, can be comparable within the "double" detection region. This statement is again confirmed at the quantitative level by the study of two benchmarks, one for colored and one for only EW interacting scalar, reported in Table 4.3 and 4.5, which show the presence of statistically relevant number of events, for both the two decay channels, in the inner detector as well as in the "outside" region. In case of identification of the two decay channels it is possible to infer the values of $\lambda$ and $\lambda^{\prime}$ as well as the $m_{\Sigma_{f}}$. The DM mass is instead not directly accessible from observations but might be determined from the requirement of the correct relic density, given its proportionality to $x=m_{\psi} / m_{\Sigma_{f}}$.

We remark anyway that the capability of disentangling the decay channels of the scalar field is actually model dependent since, according to which of the operators in (1.82) and (1.83) determine the decay processes of $\Sigma_{f}$, we might have scenarios with very different decay products in the two channels (for example in the case of $\Sigma_{\ell}$ field we can have a decay into DM and a charged lepton opposed to a decay into two jets triggered by the coupling $\lambda_{2,3} \ell$ ) as well as rather similar signals, e.g. in the case in which the scalar field decays into a neutrino and another SM fermion. We also remark that the various kinds of final states, e.g. jets, leptons etc..., possibly emerging from displaced vertices have not the same capability of reconstruction, at a given lifetime (see e.g. [116] for a discussion). In order to properly address this issue a refinement of our analysis, including a simulation of the detector, is required.

The statements discussed until now substantially hold for all the assignments of the SM charge of the $\Sigma_{f}$ particle. In absence of a detector analysis, sensitive to the decay products, the main difference is between the cases of colored or only electroweakly charged $\Sigma_{f}$ with the latter requiring higher luminosities for a discovery and featuring a more limited mass reach, in view of the lower production cross-section. In the case of colored mediator one could consider complementary searches, with respect to the one discussed in this paper, relying on the possibility of observing very late decays of $\Sigma_{f}$ particles stopped in the detector. At these very long lifetimes the observation of both decay channels of $\Sigma_{f}$ is compatible with constraints from DM phenomenology. Our study reported in Table 4.2 shows that this possibility is potentially feasible even once the actual low efficiencies in this kind of searches are accounted for. On the other hand, we remark again that in order to properly determine whether the different decay channels can be discriminated, a simulation of the detector is mandatory and that a definite statement is left to a future study. In case of possible identifications of the two decay channels it is again possible to determine their relative branching fractions and then directly the couplings $\lambda$ and $\lambda^{\prime}$.

In closing, we display in Figure 4.14 and 4.15 the CLIC reach for the direct production of long-lived $\Sigma_{\ell}$ in the parameter space $m_{\Sigma_{\ell}}-\lambda^{\prime}$. The coupling $\lambda$ has been fixed to obtain the correct DM relic density again. The $\Sigma_{\ell}$ CLIC reach for a DM mass of 10 and $100 \mathrm{GeV}$ are exhibited, respectively, on the left and right side of Figure 4.14. In Figure 4.15, on the other hand, the $\Sigma_{\ell}$ CLIC reach for a DM mass of $1 \mathrm{GeV}$ can be seen. All of these results have been achieved at $L=300 \mathrm{fb}^{-1}$ so that we can compare the latter with the corresponding LHC ones. 

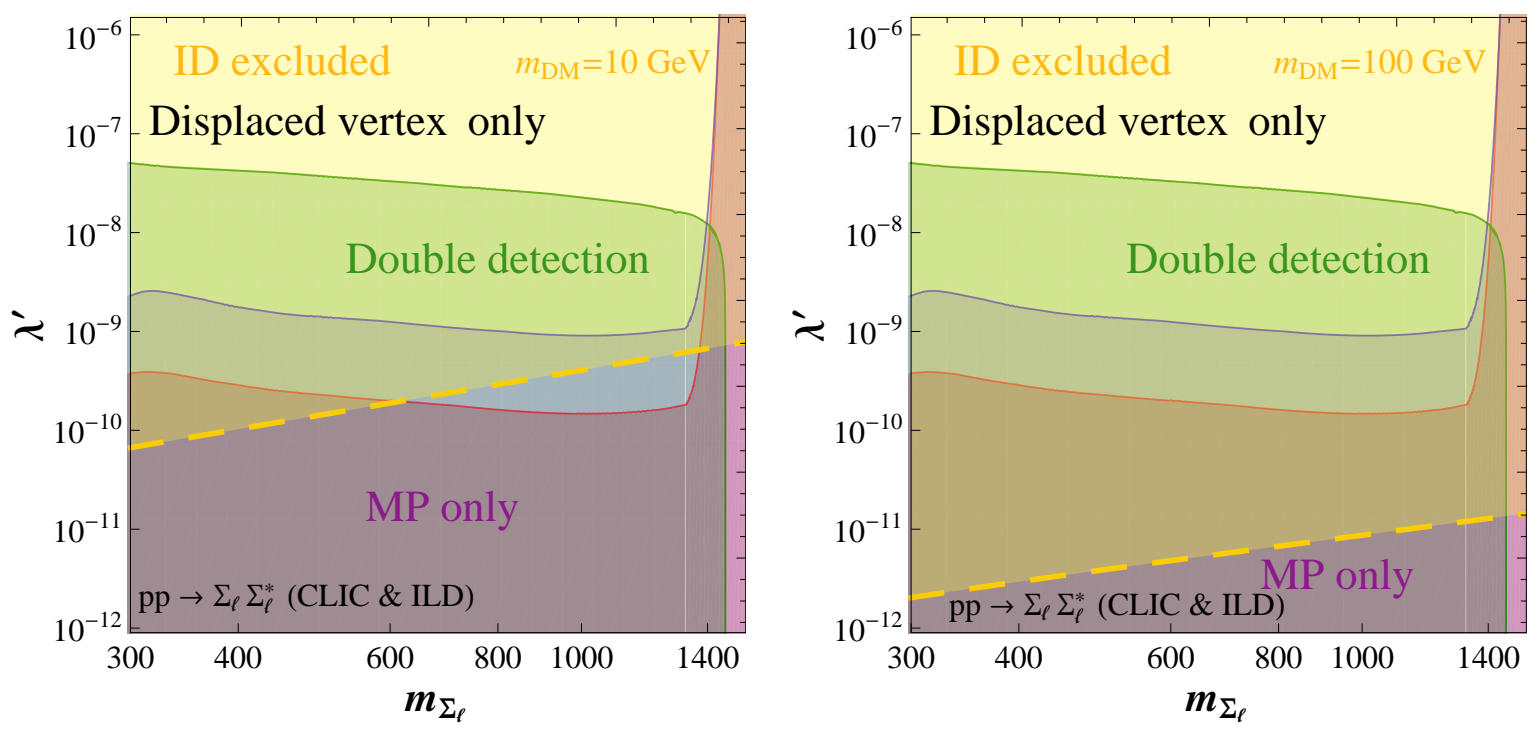

Figure 4.14: The same as Figure 4.12 but for the case of a $\Sigma_{\ell}$-type field, produced by CLIC and detected by International Linear Detector (ILD).

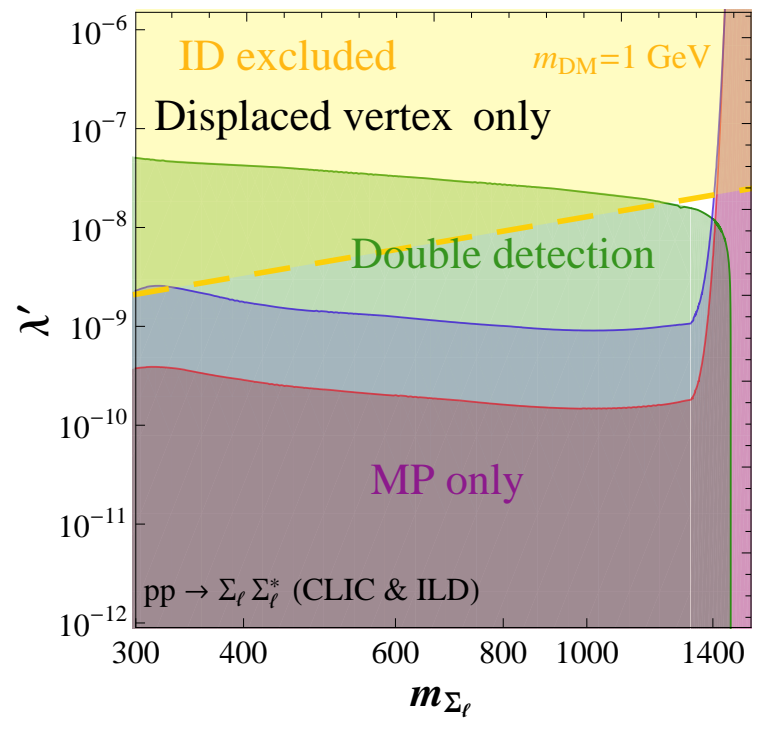

FiguRE 4.15: The same as Figure 4.14 for a DM mass of $1 \mathrm{GeV}$.

The red, blue and green lines in these plots correspond to the observation of 10 events in TPC, VTX and outside the detector. As previously denoted, the green region is for the "double" detection region. Above this region, relevant signal from displaced vertices can only be obtained, whereas below it only metastable tracks can be observed. In both plots we have again painted yellow the ID excluded region, for the considered values of the DM mass, according to the conservative bounds from ID detection used for the LHC analysis. Note that in this analysis VTX and TPC play respectively the roles of Pixel and Tracker.

By comparing the two plots of Figure 4.14 with the corresponding ones for the case of LHC collider and CMS detector, we see that the "double" detection region for CLIC is bigger than the same region for LHC. In addition, CLIC analysis investigates a region for bigger values of the coupling $\lambda^{\prime}$, thus moving the displaced vertex region up to the part of the parameter space 
already excluded by ID. This behavior can be explained by the shorter distance of the CLIC detector from the interaction point. On the other hand, CLIC analysis also investigates a region for smaller values of the coupling $\lambda^{\prime}$, thus moving the displace vertex region down to the part of the plane not excluded by ID yet. This behavior can be instead explained by the size of CLIC detector. The CLIC detector indeed has been planned to be larger than CMS and start much closer to the interaction point than CMS does. Looking at the plot for a DM mass of $10 \mathrm{GeV}$ more carefully, we note a small exception, represented by the tiny blue region. In this region, a double detection is obtained by displaced vertices in TPC and metastable particles but not by displaced vertices in VTX. Nevertheless, this region is located at big values of masses and so, most likely, we would need the full integrated luminosity of CLIC in order to investigate it. Better prospects are obtained for a DM mass of $1 \mathrm{GeV}$ since the current sensibility of ID constraints crosses the "double" detection region of Figure 4.15. Here the desired contemporary detection of the two decay channels of $\Sigma_{f}$ could be indeed obtained. Since this result strongly depends on the next constraints coming from ID, we must wait to see the destiny of this "double" detection region.

\section{Conclusions}

We have considered the LHC detection prospects of a very simple and economical extension of the Standard Model featuring a decaying Majorana fermion as DM candidate and a metastable scalar field with non-trivial quantum numbers under the SM gauge group. The potential LHC signal is constituted by the observation of the displaced decays of the scalar field, either into the DM and a SM fermion or into two SM fermions, or of the scalar field itself as disappearing track escaping the detector. We have investigated the possibility of detecting this kind of signals in the regions of the parameters space favored by the requirement of the correct DM relic density and a future Indirect Detection of the DM decays.

In the case of color charged scalar field a statistically relevant population of both displaced vertices and metastable tracks might be observed in next future $14 \mathrm{TeV}$ LHC run up to masses of the order of $1500 \mathrm{GeV}$ and up to approximately 2200 considering a high luminosity run; however bounds from DM Indirect Detection already exclude the possibility of "double" detection for DM masses above $100 \mathrm{GeV}$, leaving open only a possible detection of metastable tracks. In the parameter region corresponding to the "double" LHC detection unfortunately only the decay channel into just SM fermions is accessible to observation, since the branching ratio of the scalar field into DM is of the order $\leq 10^{-2}-10^{-3}$. The two $\Sigma_{f}$ decay channels might be instead contemporarily observed in the case of very long $\Sigma_{f}$ lifetimes, with negligible amount of events in the inner detector, considering the possibility that a fraction of $\Sigma_{f}$ can be stopped in the detector material and decay at later time. This possibility should be investigated through a dedicated study. The contemporary detection of the two decay channels of $\Sigma_{f}$ can occur as well at very low, i.e. $\ll 1 \mathrm{GeV}$, DM masses. In this case there would be the observation of mostly displaced vertices in the inner detector, while DM decays in Indirect Detection are unfortunately beyond present and future observational capabilities because of the extremely long DM lifetimes.

In the case in which, instead, $\Sigma_{f}$ features only electroweak interactions the maximal mass reach is limited to approximately $1400 \mathrm{TeV}$ and the high luminosity upgrade is most likely needed to probe the parameters space of model. Because of the lower masses of $\Sigma_{f}$ involved in the analysis the bounds from DM ID are more severe, with respect to the case of a colored scalar field, such that under the most conservative assumptions the "double" LHC detection is not possible for DM masses above $10 \mathrm{GeV}$. There are as well poorer prospects of detection 
of stopped particles because of the lower number of events and of stopping probability. The scenario of contemporary detection of the two decay channels at very low DM masses is instead feasible although kinematic effects imply, contrary to the colored case, a greater number of decays in the outer detector region. The range of possible signals is enriched in the case the scalar field is a $S U(2)_{L}$ doublet with a mass splitting between the components. In particular, in the case of $\Sigma_{\ell}$ type field, with lighter neutral component, the signals presented above can be accompanied as well by conventional missing energy signatures and prompt tracks.

In most of the parameter space of the scenario presented, LHC experiments and Indirect Detection observations provide complementary information on the model and both signals are necessary in order to confirm the identity of the Dark Matter and verify the production mechanism. In particular if the mass of the Dark Matter is in the 1-10 GeV range and the $\Sigma_{f}$ scalar within the kinematical reach of the next LHC run, i.e. below $1600 \mathrm{GeV}$ for the colored case or $1100 \mathrm{GeV}$ for the EW case, there is a clear chance to obtain multiple signals in the very near future.

At the end we have briefly investigated the phenomenology of CLIC in connection with its detection prospects of the same minimal model of decaying DM. We have only investigated the case of $\Sigma_{\ell}$ type field since its prospects are expected to be similar to those ones for $\Sigma_{d}$ due to the production channels at a lepton collider, such as CLIC. The aim is to realize if our prospects can improve by using the linear collider CLIC rather than the hadron collider LHC. We have found that although the kinematical reach at $L=300 \mathrm{fb}^{-1}$ improves so much that CLIC has the chance to cover the whole "double detection" region up to a $\Sigma_{\ell}$ mass of $1400 \mathrm{GeV}$, the DM ID bounds rule out it for both a DM mass of $10 \mathrm{GeV}$ and $100 \mathrm{GeV}$. The only possibility to preserve at least a portion of such a region is to make the DM mass smaller, i.e. $1 \mathrm{GeV}$ or $\ll 1 \mathrm{GeV}$. In this case the contemporary detection of the two decay channels of $\Sigma_{f}$ could be obtained and for $m_{D M} \sim 1 \mathrm{GeV}$ also a signal in ID DM experiments could be within the reach in the near future. 



\title{
Chapter 5
}

\section{$3.55 \mathrm{keV}$ DM line at LHC}

\author{
"The discrepancy seems to be real and important"
}

Edwin Hubble (1936)

As already stated, the nature of DM is still unknown and one of the most fundamental scientific mysteries. As a result, lately much attention has been given to anomalies/signals which can be possibly intepreted as evidence for DM interactions. One of these signals is the recently observed $3.55 \mathrm{keV} \mathrm{X}$-ray. The detection of this sharp peak at $3.55 \mathrm{keV}$ has been identified in the combined spectrum of a large set of X-ray galaxy clusters [179] as well as in the combined observation of the Perseus Cluster and the M31 galaxy [180].

A number of interpretation for this X-ray line have already been proposed. According to some of them, the $3.55 \mathrm{keV}$ line can be explained by atomic transitions (such as those associated with the chlorine or potassium ions, for example [181]), DM annihilations [182,183] and DM decays [184-216], whereas it can be hardly accounted for by astrophysical sources. Note that although the atomic interpretation is strongly justified by the fact that such a line has not been seen in stacked spheroidal galaxies or galaxy groups yet, see for instance [217,218], the viability of this explanation is currently unclear [219-221].

In this chapter we account for the above mentioned $3.55 \mathrm{keV}$ photon line in terms of decaying DM. In particular, firstly we employ the same minimal model of decaying DM used in the previous chapter and defined in Chapter 1 then we move onto two simple extensions/modifications of this scenario allowing for further couplings and fields. In the first extension we consider the case in which the DM decays into a photon and a new SM singlet, rather than the neutrino. This new state is required to be extremely light, $O(\mathrm{eV})$, and can then contribute to the number of light species $N_{\text {eff }}$ which are probed by CMB experiments. Finally in the second extension we consider the case in which the DM plays the role of a sterile neutrino. Although in this case the line signal is accounted for by the mixing with SM neutrinos, the presence of the scalar field is still relevant since it provides an additional DM generation mechanism and lightens some of the cosmological tensions characteristic of this kind of scenarios. In these scenarios we want to investigate whether an interplay between collider signals and DM indirect detection can be 


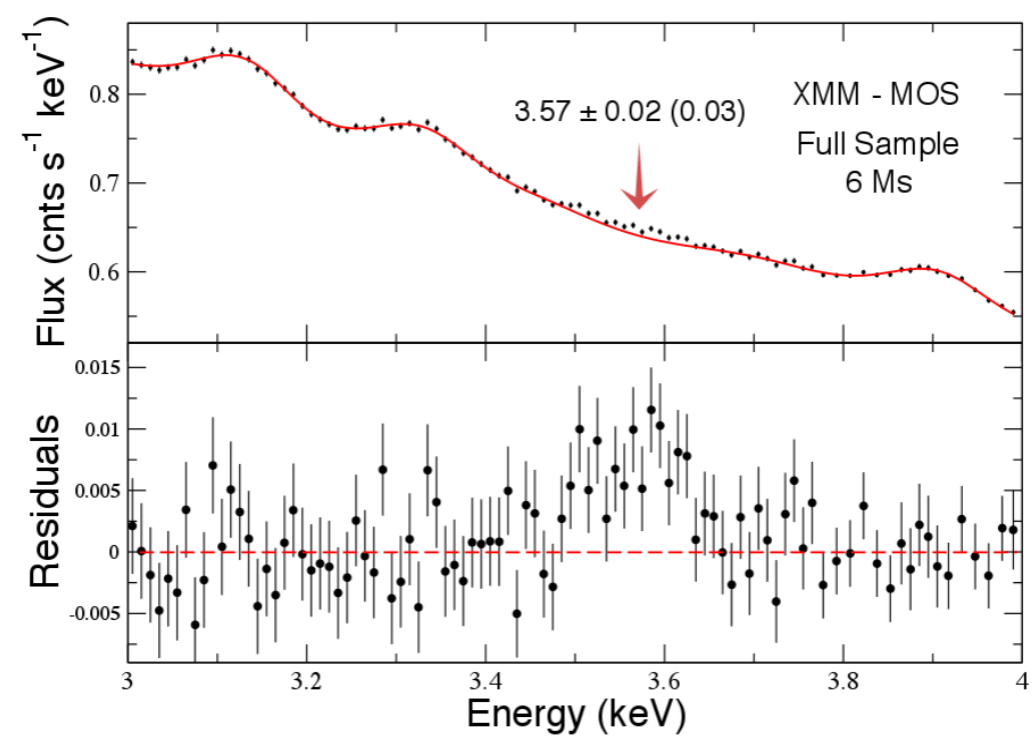

Figure 5.1: Top panel: Flux expected (red solid line) and data (black dots). Bottom panel: Residuals, which represent the differences between the data (black dots) and the expected signal (red dashed line) [179].

established for DM masses at the keV scale, for which only two-body, one-loop induced, decays into a neutrino and a photon are possible.

The chapter is organized as follows. In the next section we investigate the impact on the parameter space of the combined requirement of the agreement with the X-ray signal and of the correct DM relic density. In section 5.3 and 5.4, we present the two simple extensions mentioned above and finally discuss our conclusions.

\subsection{Experimental evidence of the $3.55 \mathrm{keV}$ line}

Before going into the description of the several extensions of the "minimal decaying DM model" and their results, we want to show the experimental evidence of the $3.55 \mathrm{keV}$ monochromatic line, detected by Bulbul and collaborators in a stacked spectrum of 73 galaxy clusters through the X-ray Multi-Mirror Mission (XMM-Newton) [179]. The two different Photon Imaging Cameras (MOS, PN) on board independently have shown the presence of a line at consistent energies. In Figure 5.1 we specifically see such a line in the rebinned MOS spectrum of the full sample, i.e. $8.5 \times 10^{6}$ source counts in the 6 Ms MOS spectra obtained after the stacking process.

On the bottom part of Figure 5.1, the residuals (measuring of the differences between each data point and the expected curve, represented by the dashed red horizontal line) as a function of energy can be seen in terms of black dots. On the top part, the flux of this line has instead been displayed. The red line denotes the expected flux while the dark dots the collected data. Here we also have a red arrow which points the excess flux registered by the experiment, and so makes the disagreement between the data and observed line clear in both parts of the figure.

This line is also detected at the same energy in the Chandra ACIS-S and ACIS-I spectra of the Perseus cluster, with a flux consistent with XMM-Newton. However, it is not seen in the ACIS-I spectrum of Virgo [179].

Since this is a blind search, in which the line energy is not known a priori, to estimate the significance of their detection, almost 70 independent energy resolution elements in the search 

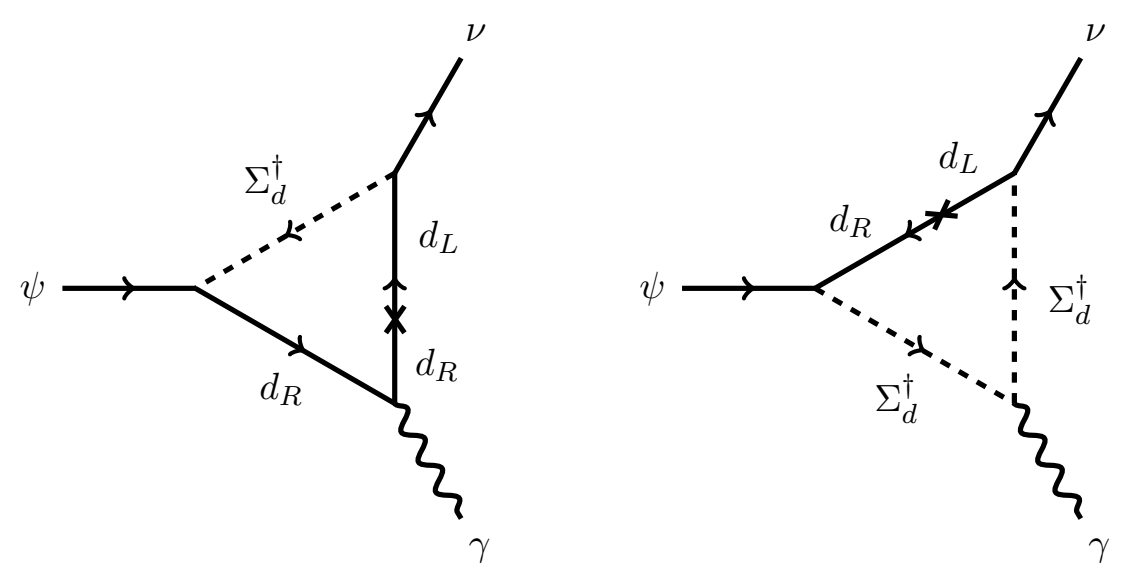

FiguRE 5.2: DM 2-body decay into $\gamma$ and $\nu$ with the loop induced by $\Sigma_{d}$.

band have been examined in [179] and eventually the $3.55 \mathrm{keV}$ emission line has been found in each of these bins. Even taking into account the probability of finding a line in each energy bin (i.e. the "look-elsewhere" effect), the statistical probability of a false detection of such a $4 \sigma$ $5 \sigma$ line at the same energy in two statistically independent data sets is negligibly small. For instance it is $0.004 \%$ for MOS.

For a more detail discussion about we refer directly to the manuscript [179].

\subsection{Minimal scenario}

As already mentioned, the first interpretation of this photon line employs the minimal model of Chapter 1. Thus we invite to go back to this chapter for a full model description. Here we only want to remind that in this simple and economic model the Majorana DM candidate couples with a scalar $\Sigma_{f}$ and a SM fermion $f$ via the coupling $\lambda$ according to the Equation (1.82). Moreover, in absence of symmetries protecting the DM stability, similar interactions are also allowed between $\Sigma_{f}$ and two SM fermions, as reported in Equation (1.83).

In this section we study the $3.55 \mathrm{keV}$ photon line, produced by the DM decay process into a photon and a neutrino and induced at one loop by the Feynman diagrams of Figure 5.2. This kind of process has a sizable branching ratio only when tree-level decays into 3 SM fermions are kinematically forbidden. The energy of the photon emitted per DM decay is given by kinematics and simply reads: $E_{\gamma}=\frac{m_{\psi}}{2}$. Therefore the DM mass is fixed to about $7 \mathrm{keV}$ if one wants to explain the recently observed X-ray line.

This kind of decay occurs only if the following couplings between the scalar field and SM fermions are present:

$$
\begin{array}{lrl}
L_{\text {eff }}=\lambda^{\prime} \bar{d}_{R} \ell_{L} \Sigma_{q}+h . c . & \Sigma_{q}=(3,2,1 / 3) \\
L_{\text {eff }}=\lambda^{\prime} \bar{\ell}_{R}^{c} q_{L} \Sigma_{d}^{\dagger}+h . c . & \Sigma_{d}=(3,1,-2 / 3) \\
L_{\text {eff }}=\lambda^{\prime} \bar{\ell}_{R}^{c} \ell_{L} \Sigma_{e}^{\dagger}+h . c . & \Sigma_{\ell}=(1,2,-1) \\
L_{\text {eff }}=\lambda^{\prime} \bar{e}_{R} \ell_{L} \Sigma_{\ell}+h . c . & \Sigma_{e}=(1,1,-2) .
\end{array}
$$

Besides the ones shown in (5.1) other operators coupling the scalar field with two SM fermions can be in general present but have no impact in the analysis performed in this work, as long as they are of the same order of magnitude or smaller than $\lambda^{\prime}$. We highlight however that all 
these operators violate baryon or lepton number, and so if both these two quantum numbers are violated very severe constraints from proton decay may arise. Thus, we implicitly assume throughout all this work that there is no contemporary presence of operators which violate $B$ and $L$ numbers. In addition, we also assume, similarly to the previous chapter, that possible couplings of the scalar field with the SM Higgs, not forbidden by gauge interactions, like e.g. $\lambda_{h h \Sigma \Sigma}|H|^{2}\left|\Sigma_{f}\right|^{2}, \lambda_{h h \Sigma \Sigma}(H \Sigma)^{\dagger}(H \Sigma)$ or, in the case of $\Sigma_{l}$-type field only, $\lambda_{h \Sigma} H \Sigma_{l}$ (with $\lambda_{h \Sigma}$ being a dimensionfull coupling), are set to zero. Indeed, although this kind of couplings does not affect the relevant processes in this work, it might influence the mass of the scalar field. We will come back to this point in the next sections. In the case of $\Sigma_{\ell}$-type field only, a coupling $\lambda_{h \Sigma} H \Sigma_{\ell}$ (with $\lambda_{h \Sigma}$ being a dimensionful coupling) could also arise, which could generate a mixing between the scalar field and the Higgs field and also a non-vanishing v.e.v. for $\Sigma_{\ell}$. We disregard this possibility in order to keep a single purely Standard-Model-like Higgs field. Note that for what regards DM phenomenology a non-vanishing $\Sigma_{\ell}^{0}$ v.e.v. mixes DM with the neutrinos in a very similar way as discussed in Section 5.4.

The decay rate of the DM into a neutrino and a photon is given by [40]:

$$
\begin{gathered}
\Gamma(\psi \rightarrow \gamma \nu)=\frac{e^{2} m_{\psi}^{3}}{2048 \pi^{5}}\left(\sum_{i} \frac{m_{i}}{m_{\Sigma_{f}}^{2}} \lambda_{i}^{\prime} \lambda_{i} f_{1}\left(\frac{m_{i}^{2}}{m_{\Sigma_{f}}^{2}}\right)\right)^{2}, \\
\text { where } f_{1}(x)=\frac{1}{1-x}\left[1+\frac{1}{1-x} \ln x\right]
\end{gathered}
$$

and the sum runs over the fermions flowing inside the loop. We notice that the decay rate depends on the mass of the SM fermion in the loop since a chirality flip in the internal fermion line is required in order to have a photon and a neutrino in the final states. Unless particular hierarchies in the couplings $\lambda$ and $\lambda^{\prime}$ with respect to the fermion flavors are assumed, the DM decay rate is mostly sensitive to the couplings of $\Sigma_{f}$ with third generation fermions. For this reason, unless differently stated, from now on we assume the couplings $\lambda$ and $\lambda^{\prime}$ flavor universal and keep only the contribution coming from the third generation.

It is easy to see that the maximal value of the rate is achieved in the case of a bottom quark running in the loop. In fact, due to the quantum numbers of the SM neutrino, it is impossible to construct a loop with an intermediate top quark. By taking $m_{b}=4 \mathrm{GeV}$, the lifetime of the DM in this case can be estimated as:

$$
\tau(\psi \rightarrow \gamma \nu) \simeq 5.6 \times 10^{6} \mathrm{~s}\left(\frac{m_{\psi}}{7 \mathrm{keV}}\right)^{-3}\left(\frac{m_{\Sigma_{f}}}{1 \mathrm{TeV}}\right)^{4}\left(\lambda \lambda^{\prime}\right)^{-2} .
$$

Now if we require a value of the DM lifetime of $10^{28} \mathrm{~s}$, as expected for the detected photon line, the condition:

$$
\lambda \lambda^{\prime} \simeq 2.4 \times 10^{-11}\left(\frac{m_{\psi}}{7 \mathrm{keV}}\right)^{-3 / 2}\left(\frac{m_{\Sigma_{f}}}{1 \mathrm{TeV}}\right)^{2}\left(\frac{\tau(\psi \rightarrow \gamma \nu)}{10^{28} \mathrm{~s}}\right)^{-1 / 2} .
$$

is obtained. As evident from this equation the prediction for the value of the product $\lambda \lambda^{\prime}$ is much higher than the one considered in the preceding chapter and even before in [8]. This is consequence of the strong sensitivity of the DM lifetime on the DM mass. We can determine the single values of the two couplings $\lambda$ and $\lambda^{\prime}$ by combining Equation (5.4) with the requirement 
of the correct relic DM relic density. Indeed the latter is determined by a combination of freezein [102] and SuperWIMP $[98,222]$ mechanisms, both relying on the decay of the scalar field into DM, as given by Equation (2.49).

The abundance of the scalar field is fixed by interactions mediated by its gauge couplings and is not influenced by the couplings $\lambda$ and $\lambda^{\prime}$. As shown in [8], the contribution of the SuperWIMP mechanism is negligible when $m_{\psi} \ll m_{\Sigma_{f}}$. It is then possible to directly relate the value of the DM relic density to the coupling $\lambda$ through the Equation (4.3), which can be rewritten by using the normalization of the DM mass to $7 \mathrm{keV}$ as:

$$
\lambda \simeq 0.8 \times 10^{-8}\left(\frac{m_{\psi}}{7 \mathrm{keV}}\right)^{-1 / 2}\left(\frac{m_{\Sigma_{f}}}{1 \mathrm{TeV}}\right)^{1 / 2}\left(\frac{g_{*}}{100}\right)^{3 / 4}\left(\frac{\Omega h^{2}}{0.11}\right)^{1 / 2} .
$$

As discussed in Section 2.7, in this minimal model of decaying DM the general upper bound on the coupling $\lambda$ given by Equation 2.45 can be easily obtained by the requirement that DM is out of the equilibrium in the early Universe. For the result presented below, this upper bound can be expressed as:

$$
\lambda \lesssim 0.6 \times 10^{-7}\left(\frac{m_{\Sigma_{f}}}{1 \mathrm{TeV}}\right)^{1 / 2}
$$

if we set $g_{\Sigma}=6$ (value for the $\Sigma_{d}$ scalar representation) in Equation 2.45.

Substituting Equation (5.5) into Equation (5.4) we can simply determine the value of the coupling $\lambda^{\prime}$ :

$$
\lambda^{\prime} \simeq 3 \times 10^{-3}\left(\frac{m_{\psi}}{7 \mathrm{keV}}\right)^{-1}\left(\frac{m_{\Sigma_{f}}}{1 \mathrm{TeV}}\right)^{3 / 2}\left(\frac{\tau(\psi \rightarrow \gamma \nu)}{10^{28} \mathrm{~s}}\right)^{-1 / 2}
$$

According to this result we see that there is a much stronger hierarchy between the couplings $\lambda$ and $\lambda^{\prime}$ with respect to the one found in the previous chapter and in [8] as well. Indeed, in order to compensate the suppression of the decay rate due to the DM mass of the order of $\mathrm{keV}$, we can only increase the coupling $\lambda^{\prime}$ since the coupling $\lambda$, instead, fixed by the freeze-in mechanism as in Equation (5.5), is a rather slowly varying function of the DM mass and is still very suppressed.

In Figure 5.3 the value of the coupling $\lambda^{\prime}$ given by Equation (5.7), as function of the mass $m_{\Sigma_{f}}$ of the scalar field (taken to be of $\Sigma_{d^{-}}$type as clarified below), has been reported via a solid blue line. For this value of $\lambda^{\prime}$ it is possible to obtain a decay length for the scalar field of:

$$
l_{\Sigma_{f}} \simeq 5.6 \times 10^{-11} \mathrm{~cm}\left(\frac{m_{\psi}}{7 \mathrm{keV}}\right)^{2}\left(\frac{m_{\Sigma_{f}}}{1 \mathrm{TeV}}\right)^{-4}\left(\frac{\tau(\psi \rightarrow \gamma \nu)}{10^{28} \mathrm{~s}}\right) .
$$

which implies a scalar field promptly decaying into SM particles only. At this stage it is important to highlight that, due to the dependence of Equation (5.2) on the internal fermion mass, the value of $\lambda^{\prime}$ reported in Equation (5.7) is the minimal achievable. The conclusion above hence is valid for all the realizations given in Equation (1.83). For this reason we are going to focus from now on, for definiteness, on the case of $\Sigma_{d^{-}}$type field.

Figure 5.3 also shows, as dashed blue line, the value of $\lambda^{\prime}$ that would be obtained by combining Equation (5.4) with the condition (5.6) of not equilibrium for the DM. As evident, this value is always more than two orders of magnitude higher than the one needed for observable displaced decays (violet region). As consequence, a cosmologically viable scenario compatible with the 


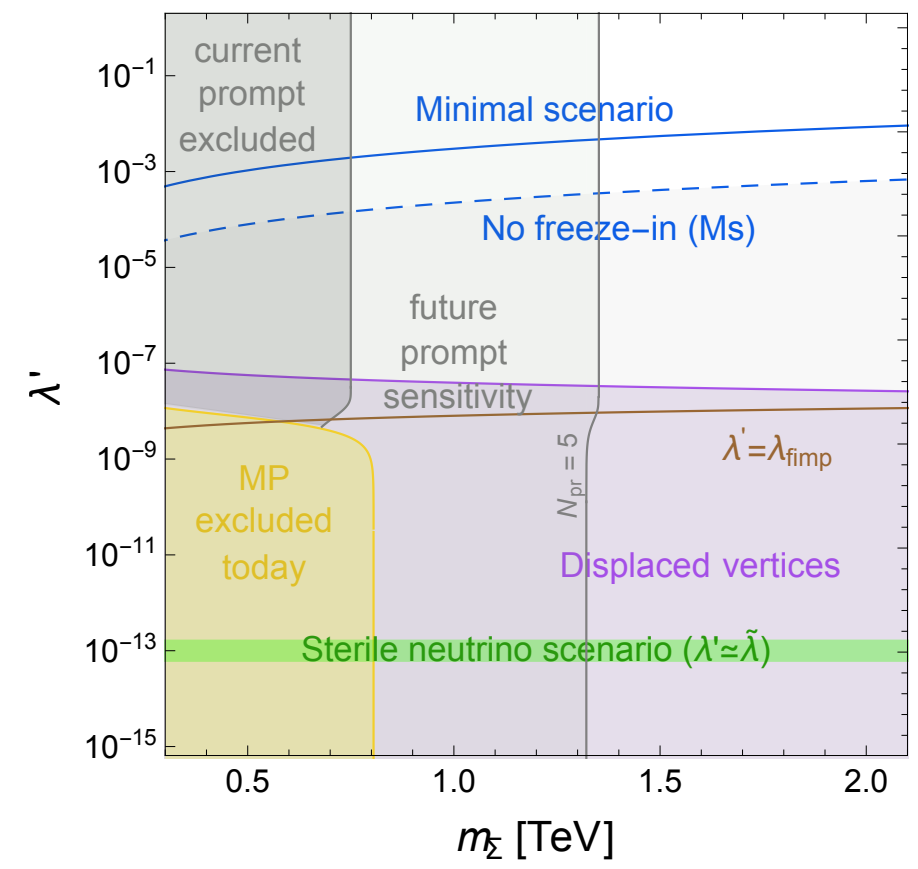

FiguRE 5.3: Summary plot for the minimal decaying dark matter scenario. The blue solid and dashed lines correspond to the required values of $\lambda^{\prime}$ in order to match the experimentally favored DM lifetime for the values of $\lambda$ given by, respectively, Equation (5.5) (correct DM relic density) and (5.6) (out-of-equilibrium condition). The violet region corresponds to a long-lived scalar field, decaying though displaced vertices or even outside the LHC detector. The gray and yellow regions are excluded by present $L H C$ searches while in light gray we give the expected exclusion in case less than five prompt decays will be detected in the next LHC run after collecting 100 $f b^{-1}$ (see text for details). For reference, we show as well with a brown line the case $\lambda^{\prime}=\lambda$ and with a green band the case $\lambda^{\prime}=\tilde{\lambda}$, both relevant in the scenario, described in Section 5.4, where the X-ray line is generated by the coupling of the DM with the SM Higgs and the lepton doublet, given as $\tilde{\lambda} H l$.

generation of the observed $3.55 \mathrm{keV}$ photon line from the decay of DM necessarily implies a strong hierarchy between the couplings $\lambda$ and $\lambda^{\prime}$, and therefore prompt decays of the scalar field into only SM states. On the other hand we remark that this requirement relies on the assumption of a standard cosmological evolution and can be relaxed in non-standard cosmological scenarios where, for example, entropy injection occurs during the phase of DM generation [223,224]. The brown line in Figure 5.3 shows instead the scenario with comparable values of the couplings $\lambda$ and $\lambda^{\prime}$, thus giving, for $m_{\Sigma_{d}} \gtrsim 750(1300)$ at $\sqrt{s}=7(14) \mathrm{TeV}$, a long-lived (on detector scales) scalar field, with both types of decay channels, namely DM+SM and only SM, potentially observable at LHC. Unfortunately such a case corresponds to a DM lifetime of the order of $O\left(10^{38}\right)$ s which is incompatible with the observed X-ray line and far beyond the sensitivity of future ID experiments.

Contrary to the scenarios discussed in the preceding chapter and in [8], for the case of $\mathrm{keV} \mathrm{DM}$, the cosmological viability enforces the prediction of a promptly decaying scalar field with at least two possible decay channels into a third generation quark and a neutrino or a charged lepton, arising through the same coupling constant $\lambda^{\prime}$. The conventional constraints from LHC searches then apply. In the case, under consideration, of a color charged scalar field the relevant bounds come from searches of leptoquarks. The most severe limits have been, at the moment, set by CMS excluding for the scenario under consideration masses of the 
scalar field below approximately $840 \mathrm{GeV}$ [53]. This limit is relaxed down to $740 \mathrm{GeV}$ [225] if coupling with only third generation fermions is assumed. We have determined, according to these limits, the excluded region in the plane $\left(m_{\Sigma_{f}}, \lambda^{\prime}\right)$ (the coupling $\lambda$ has been set by requiring the correct DM relic density as in Equation (5.5)), by focussing for simplicity on the case of coupling between the scalar and only third generation fermions. In more detail, we have used the package Madgraph $[144,172]$ to simulate the pair production of the scalar field $\Sigma_{d}$ at LHC and subsequently we have computed analytically the spatial distribution of the decay events. Note that in this computation we also have taken into account the the detector efficiency, as function of $m_{\Sigma_{d}}$, reported in [225]. In order to apply LHC searches of prompt decays we have imposed the detection of at least 5 events, corresponding to the expected signal for a leptoquark with mass of $750 \mathrm{GeV}$, before the pixel region of the detector (see the two previous chapter for some more details). The excluded region determined through this procedure corresponds to the dark gray region in Figure 5.3 and, as can be noticed, is in good agreement with the experimental exclusion as regards the mass of $\Sigma_{d}$. It extends down to values of $\lambda^{\prime} \approx 10^{-(7 \div 8)}$ (the coupling $\lambda$ has been kept fixed according to Equation 5.5). At lower values of $\lambda^{\prime}$ leptoquark searches are complemented by searches for metastable particles [51,122], shown in Figure 5.3 by the yellow region. An analogous analysis can be done for the case of only electroweakly interacting scalar field. The most suitable searches for this scenario are the ones of supersymmetric particles decaying into leptons and missing energy. From these searches, one can infer a lower limit on the mass of the scalar as $m_{\Sigma_{\ell, e}}>160-200 \mathrm{GeV}$, as claimed in $[173,174,226,227]$.

We have in addition determined the expected sensitivity for next LHC run at $14 \mathrm{TeV}$ of centre of mass energy and for a luminosity of $100 \mathrm{fb}^{-1}$. We have thus generated pair production events of $\Sigma_{d}$, assuming the same experimental efficiency as in the $8 \mathrm{TeV}$ searches and requiring again at least 5 detected events. The region of sensitivity of future searches is reported as a light gray region in Figure (5.3). Note that those searches for prompt decays can probe arbitrary low values of $\lambda^{\prime}$, since even for negligibly small $\lambda^{\prime}$, there is a substantial decay rate set by the coupling $\lambda$, given by (5.5). Although for this value of the coupling, the naive expectation, i.e $l_{\Sigma_{f}}=c \tau_{\Sigma_{f}}$, of the decay length corresponds to displaced vertices, as shown in Figure 5.3 by the brown line, it is near to the boundary with prompt decays and once the proper statistical distribution of the decay events is taken into account, a residual number of prompt decays, within the reach of experimental searches, is nonetheless present. This does not occur for larger DM masses, as studied earlier in this thesis, since in this case the value of $\lambda$ is several orders of magnitude below that considered here and guarantees the absence of observable prompt decays. The LHC searches in the next run will then probe masses of $\Sigma_{d}$ up to approximately $1400 \mathrm{GeV}$ (a higher mass reach can be achieved by considering a high luminosity upgrade of LHC with $O(1000) \mathrm{fb}^{-1}$ of luminosity), covering a wider parameter region compared to that where the $\mathrm{X}$-ray line can be explained by DM decay.

As evident in the discussion, also for a $\mathrm{keV}$ DM the combined requirement of an ID DM signal and the correct relic density establishes rather definite prospects for an eventual LHC detection of the scalar field. We might then ask whether an hypothetical LHC detection, combined with the X-ray signal, allows an unambiguous determination of the relevant parameters and a clear discrimination with respect to other particle physics models. The clearest LHC signature would be the contemporary detection of the two different kinds of decay channel of the scalar field, through the two couplings $\lambda$ and $\lambda^{\prime}$. Unfortunately this result is not achievable since, as we can see from Equation (5.5) and (5.7), the decay channel into DM has a too suppressed branching ratio to be observable. A possibility of inferring the parameters of the model would nonetheless 
occur if the LHC detection of the decay into two SM fermions would allow the reconstruction of the mass of the scalar field and the measurement of its lifetime. Indeed these two information, combined with the ID of the X-ray line (which provides the DM mass and the value of $\lambda \lambda^{\prime}$ as function of $m_{\Sigma_{f}}$ ), would allow the individual determination of the parameter $\lambda$, which could be used to test the FIMP paradigm. This task is however very challenging since the coupling (5.7) corresponds to a decay width of the order of few $\mathrm{MeVs}$, which is much below the resolution, $O(\mathrm{GeV})$, of the LHC detector. For the sake of completeness, we point out that recently it has been shown e.g. in [228] that it is in principle possible to probe decay widths below these energy resolutions. Although most probably values of the order of (5.7) are accessible only to precision machines, the LHC measurements can potentially set upper bounds down to approximately one order of magnitude above the expected value. In addition one could adopt a similar procedure to the one used for determining the exclusion region from leptoquark searches to look for the presence of residual displaced vertices in the case of mostly promptly decaying states. Non observation of these events would also allow to set a lower bound on $\lambda^{\prime}$ which, combined with the previous upper bound, could determine a window of allowed values of $\lambda$ to be compared with the value needed for FIMP production testing its viability. On the contrary the observation of displaced vertices could be translated into a strong upper bound on $\lambda^{\prime}$ which might possibly rule out our cosmological framework.

We remark that the picture depicted above is strictly valid only in the case if the scalar field is a $\mathrm{SU}(2)$ singlet. In the doublet case we are implicitly assuming that the two components are exactly degenerate in mass. If this is not the case additional signals originated by the production and decay of the heavier components might results detectable. In particular we would have decays into the lighter component and a W boson (either on- or off-shell). These processes are determined by gauge interactions and cannot be directly related to DM phenomenology. This issue is particularly relevant in the case of $\Sigma_{\ell}$-type fields since the dominant production process is, in general, $p p \rightarrow \Sigma_{\ell}^{0} \Sigma_{\ell}^{ \pm}$, where $\Sigma_{\ell}^{0}$ and $\Sigma_{\ell}^{ \pm}$are, respectively, the charged and the neutral component of the doublet.

\subsection{Dark matter and Dark radiation scenario}

In this section we discuss the first extension of the minimal decaying DM scenario studied in the previous section. Specifically, in this extension the spectrum of the Beyond Standard Model (BSM) states is augmented with another SM singlet $\chi$ and the scalar spectrum is constituted by two fields, a $\mathrm{SU}(2)$ doublet and a singlet. The photon line is now produced by the decay $\psi \rightarrow \chi \gamma$, whose Feynman diagram has been drawn in Figure 5.4. Such a decay is described by the following lagrangian:

$$
\begin{aligned}
L_{\mathrm{eff}}= & \left(\lambda_{\mathrm{L}} \bar{\psi} q_{L} \Sigma_{q}^{\dagger}+\lambda_{\mathrm{R}} \bar{\psi} t_{R} \Sigma_{u}^{\dagger}\right)+\text { h.c. } \\
& +\left(\lambda_{L}^{\prime} \bar{\chi} q_{L} \Sigma_{q}^{\dagger}+\lambda_{R}^{\prime} \bar{\chi} t_{R} \Sigma_{u}^{\dagger}+\text { h.c. }\right) \\
& +\mu H \Sigma_{q} \Sigma_{u}^{\dagger}+\text { h.c. }
\end{aligned}
$$

We have assigned to the two scalar fields the quantum numbers of a left-handed and righthanded up-quark in order to enhance the loop function through the top mass and possibly achieve the desired value of the DM lifetime for suppressed couplings, such that the scalar fields are long-lived at the LHC. Given the strong sensitivity of the DM lifetime on the SM fermion 

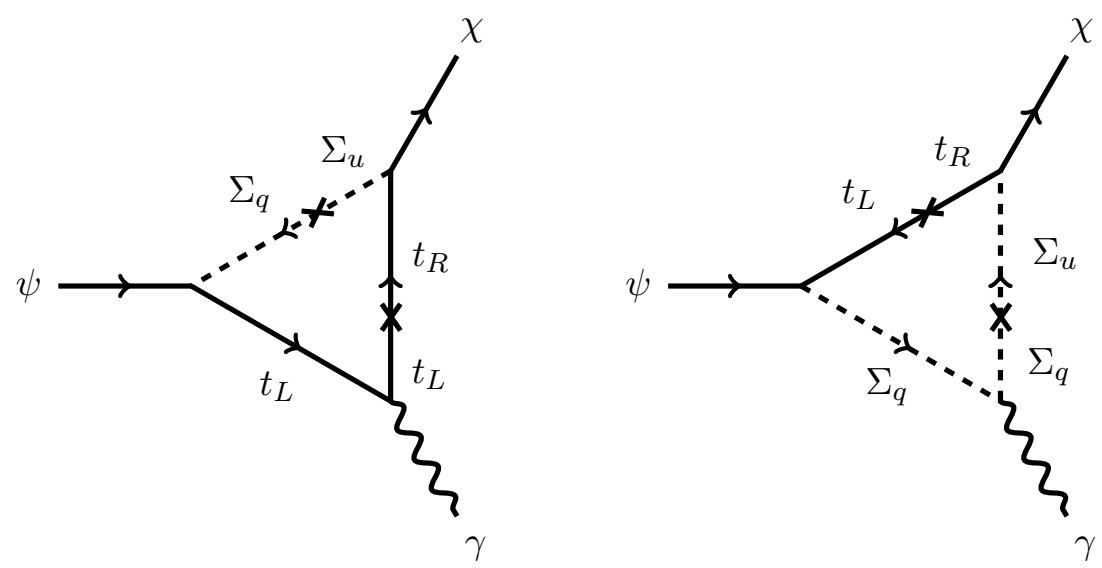

FIGURE 5.4: Diagrams contributing at one-loop to the DM 2-body decay into $\gamma$ and $\chi$ induced by scalar-mixing.

masses, we have assumed for simplicity that the two SM singlets $\chi$ and $\psi$ are coupled only with the third generation quarks. The couplings of the scalar field with only SM fermions, which we have omitted for simplicity, are not relevant for the DM decay and they can be set to be of comparable value to the couplings governing the decay of the scalar field into DM.

In this case after the electroweak symmetry breaking the fields $\Sigma_{q}, \Sigma_{u}$ mix with each other, thus giving the physical fields $\Sigma_{1}, \Sigma_{2}$, which are the eigenstates of the mass matrix:

$$
\mathcal{M}=\left(\begin{array}{cc}
m_{\Sigma_{u}}^{2} & \mu v \\
\mu v & m_{\Sigma_{q}}^{2}
\end{array}\right)
$$

where $v$ is the v.e.v. of the Higgs field. The eigenvalues of the matrix $\mathcal{M}$ are instead given by the expression:

$$
m_{\Sigma_{1,2}}^{2}=\frac{1}{2}\left(m_{\Sigma_{q}}^{2}+m_{\Sigma_{u}}^{2} \mp \sqrt{\left(m_{\Sigma_{q}}^{2}-m_{\Sigma_{u}}^{2}\right)^{2}+4 v^{2} \mu^{2}}\right),
$$

and they can be obtained by diagonalizing $\mathcal{M}$ through the generic matrix:

$$
\eta=\left(\begin{array}{cc}
\cos \theta & \sin \theta \\
-\sin \theta & \cos \theta
\end{array}\right)
$$

where the mixing angle is given by:

$$
\tan 2 \theta=\frac{2 \mu v}{m_{\Sigma_{q}}^{2}-m_{\Sigma_{u}}^{2}} .
$$

In the above expressions $m_{\Sigma_{q}}$ and $m_{\Sigma_{u}}$ represent the mass terms of the $\mathrm{SU}(2)$ doublet and singlets. These are assumed to be originated by conventional mass terms of the form $m_{\Sigma_{q, u}}^{2} \Sigma_{q, u}^{\dagger} \Sigma_{q, u}$. As already mentioned, additional mass terms can be originated by terms, allowed by gauge symmetries, of the type $\lambda_{h h \Sigma \Sigma}|H|^{2}\left|\Sigma_{q, u}\right|^{2}$ and/or $\lambda_{h h \Sigma \Sigma}\left(H \Sigma_{q}\right)^{\dagger}\left(H \Sigma_{q}\right)$ whose contributions are $O(100 \mathrm{GeV})$ for couplings of order one. Moreover, we expect them to be subleading compared to that proportional to the dimensionful quantity $\mu$ since they do not mix the two states and just change $m_{\Sigma_{u, d}}$. A theoretical investigation of the scalar sector of the theory is beyond the scope of this work; our results will be thus expressed in terms of the mass eigenstates $m_{\Sigma_{1}}$ 
and $m_{\Sigma_{2}}$, assumed to be free parameters, and we will regard (5.10) and (5.12) as a generic parametrization.

The DM decay rate is then given by:

$$
\begin{aligned}
& \Gamma(\psi \rightarrow \chi \gamma)=\frac{\alpha m_{\psi}^{3}}{32 \pi^{4}}\left(1-\frac{m_{\chi}^{2}}{m_{\psi}^{2}}\right)^{3} \\
& {\left[m_{t} \sin \theta \cos \theta\left(\lambda_{L} \lambda_{R}^{\prime}-\lambda_{R} \lambda_{L}^{\prime}\right)\left(\frac{1}{m_{\Sigma_{1}}^{2}} f_{1}\left(\frac{m_{t}^{2}}{m_{\Sigma_{1}}^{2}}\right)-\frac{1}{m_{\Sigma_{2}}^{2}} f_{1}\left(\frac{m_{t}^{2}}{m_{\Sigma_{2}}^{2}}\right)\right)\right.} \\
& \left.-\frac{1}{4}\left(m_{\psi}-m_{\chi}\right)\left(\lambda_{L} \lambda_{L}^{\prime}-\lambda_{R} \lambda_{R}^{\prime}\right)\left(\frac{\sin ^{2} \theta}{m_{\Sigma_{1}}^{2}} f_{2}\left(\frac{m_{t}^{2}}{m_{\Sigma_{1}}^{2}}\right)+\frac{\cos ^{2} \theta}{m_{\Sigma_{2}}^{2}} f_{2}\left(\frac{m_{t}^{2}}{m_{\Sigma_{2}}^{2}}\right)\right)\right]^{2} \\
& \text { where } f_{2}(x)=\frac{1}{(1-x)^{2}}\left[1+x+\frac{2 x}{1-x} \ln x\right]
\end{aligned}
$$

The term inside the square bracket in the DM decay rate is maximized for $\theta=\frac{\pi}{4}$, i.e. $m_{\Sigma_{q}}^{2}-$ $m_{\Sigma_{u}}^{2}=0$, and $\lambda_{L}=\lambda, \lambda_{R}=0, \lambda_{L}^{\prime}=0, \lambda_{R}^{\prime}=\lambda^{\prime}$ (or vice versa). For substantial mass splitting, the DM lifetime is mostly determined by the contribution from the lightest eigenstate in that case.

Neglecting the mass of $\chi$ (the reason will be clarified in the following) it can be expressed as:

$$
\tau(\psi \rightarrow \chi \gamma) \simeq 1.4 \times 10^{4} \mathrm{~s}\left(\frac{m_{\psi}}{7 \mathrm{keV}}\right)^{-3}\left(\frac{m_{\Sigma_{1}}}{1 \mathrm{TeV}}\right)^{4}\left(\lambda \lambda^{\prime}\right)^{-2}
$$

The DM production is as well dominated by $\Sigma_{1}$ and $\lambda$ is again determined by Equation (5.5). The expected value of $\lambda^{\prime}$ from the combined requirement of reproducing the photon-line and the correct DM relic density is:

$$
\lambda^{\prime} \simeq 1.5 \times 10^{-4}\left(\frac{m_{\psi}}{7 \mathrm{keV}}\right)^{-1}\left(\frac{m_{\Sigma_{1}}}{1 \mathrm{TeV}}\right)^{3 / 2} .
$$

This value, although sensitively lower than the one obtained in the previous case, is still large enough to make the scalar field decay promptly at the LHC. Note that in the case of nearly degenerate mass eigenstates, a partial cancellation between the two contribution takes place and therefore larger couplings are required to match the same lifetime. Being substantially free parameters, the couplings of the scalar field with only SM fermions can be of comparable order as (5.16) in order to allow for the observation of a double LHC signal. Since we are still dealing with prompt decays, the strong limits from LHC searches must be taken into consideration. The most stringent ones come from searches of top squarks. In particular, current limits allow $177 \lesssim m_{\Sigma_{1}} \lesssim 200 \mathrm{GeV}$ or $m_{\Sigma_{1}}>750 \mathrm{GeV}$ [54-56]. These ranges can be actually relaxed in presence of a branching ratio of decay into missing energy lower than 1 .

We have compared, analogously to what done in the previous section, in Figure 5.5 the prospects of LHC detection with the information from DM phenomenology. The scenario depicted is analogous to the minimal scenario of the previous section with the (standard) cosmology that strongly prefers prompt decays. Despite the different experimental signature (top plus missing energy) the prospects of hypothetical LHC detection are as well very similar to the previous scenario. On the other hand, within the assumption of no extra symmetries with 


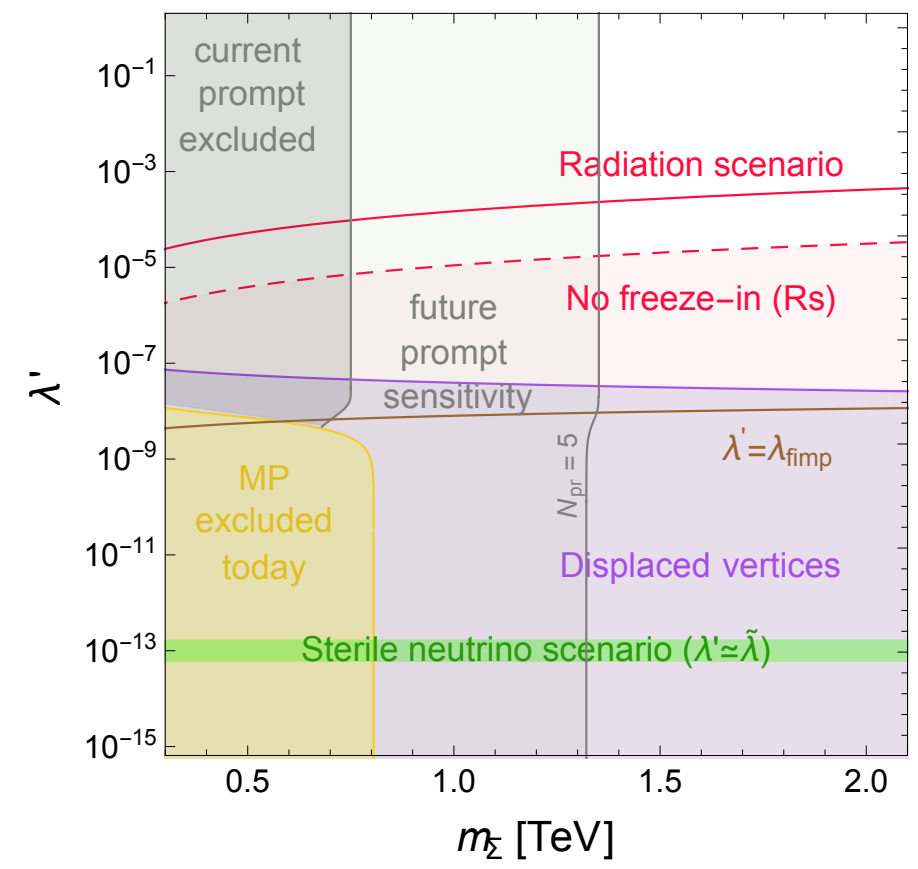

FIGURE 5.5: The same as Figure 5.3 but for the DM+Dark Radiation scenario. We give here as red solid and dashed lines the values of the coupling $\lambda^{\prime}$ corresponding to the right DM lifetime and FIMP production and to the out-of-equilibrium condition respectively. The other lines are as in Fig. 5.3. Notice that the limits and the future sensitivity for prompt decays refer here to searches for supersymmetric top partners decaying into top quarks and missing energy.

respect to the SM, a coupling between the scalar field and only SM fermions is allowed and can be of the same order as $\lambda^{\prime}$, providing then two types of decays and signatures (low and high amount of missing energy) for the scalar field. As already mentioned in the previous section, this discussion is strictly valid under the assumption that LHC phenomenology is dominated by a single heavy charged state. In case also the heavier mass eigenstate $\Sigma_{2}$ is efficiently produced we would have additional signals like the one studied in [229] which could not be directly correlated with DM signals. We remind, on the other hand, that the two scalar fields $\Sigma_{q}$ and $\Sigma_{u}$ are mostly produced at the LHC through gluon fusion and their production cross-section is strongly sensitive to the mass of the pair-produced states. Our scenario requires a sizable value of $\mu v$, in order not to suppress the mixing angle, implying a sizable mass splitting between the two mass eigenstates $\Sigma_{1}$ and $\Sigma_{2}$. The assumption that the dominant LHC signals are mostly related to $\Sigma_{1}$ appears thus reasonable.

Notice also that the state $\chi$ is cosmologically stable if it is very light and might give a sizable contribution to the overall DM abundance. Indeed, contrary to the case of $\psi$, the value of the coupling $\lambda^{\prime}$ is high enough to create, at early stages of the history of the Universe, a thermal population of $\chi$ particles through decays/inverse decays of the scalar fields and the $2 \rightarrow 2$ scattering processes with top quarks. In that moment the $\chi$ particles undergo a relativistic freeze-out at temperatures between $100 \mathrm{GeV}$ and $1 \mathrm{TeV}$. In order to avoid bounds from overclosure of the Universe and structure formation, we impose a very small mass for this new state, $m_{\chi} \lesssim O(\mathrm{eV})$. Such a light state would then remain relativistic for a long time and contribute to the number of effective neutrinos $N_{\text {eff }}$. The deviation from the SM prediction $N_{\text {eff }}=3.046$ induced by the 

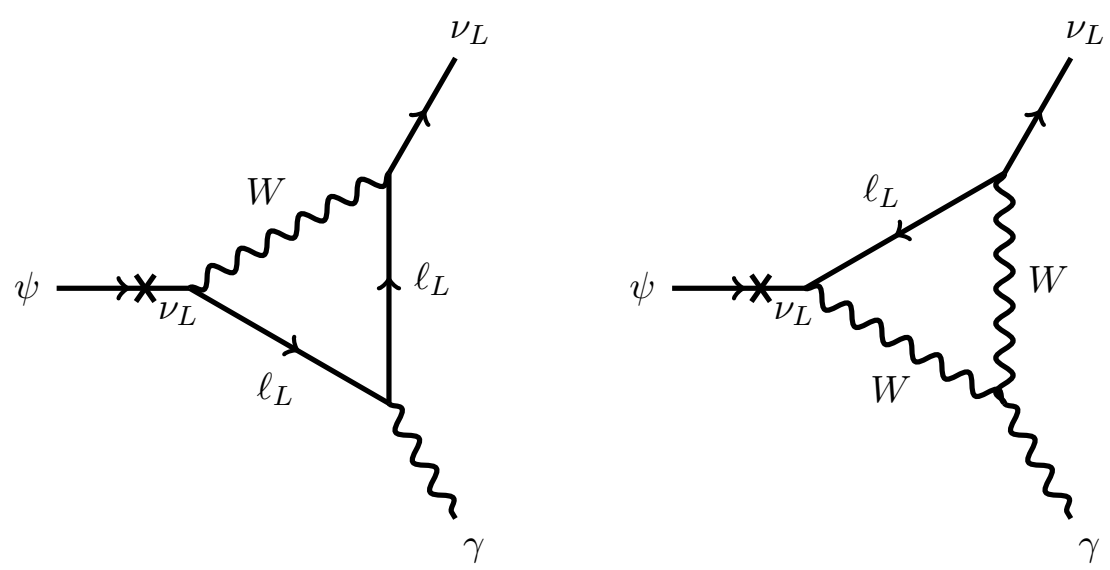

Figure 5.6: Diagrams contributing at one-loop to the DM 2-body decay into $\gamma$ and $\nu$ induced by $W$.

$\chi$ particles can be expressed as $[230,231]$ :

$$
\Delta N_{\mathrm{eff}}=\frac{23.73}{\left(g_{*}^{s}\left(T_{d}\right)\right)^{4 / 3}},
$$

where $T_{d}$ represents the decoupling temperature from the primordial thermal bath of the $\chi$ particles. Thanks to the rather high decoupling temperature, we have $g_{*}^{s}\left(T_{d}\right) \sim 100$ due to Standard Model states and therefore $\Delta N_{\text {eff }} \sim 0.05$, which is compatible with the current constraints [232]. Unfortunately such a small contribution to the number of relativistic species is at the boundary of detection even for an ideal CMB experiment including polarization [233].

Before moving onto our last DM decay scenario, it is worth remarking the direct correlation of the X-ray signal with the presence of one of the SM singlets in our scenario in thermal equilibrium. Indeed, in order to the ensure the desired lifetime of the DM we need $\lambda \lambda^{\prime} \sim 10^{-12}$. Comparing this value with Equation (5.6) we notice that the two couplings cannot contemporary satisfy the out-of-equilibrium condition.

\subsection{DM as sterile neutrino}

The second and last extension of the minimal decaying DM scenario of Section 5.2, capable of reproducing the $3.55 \mathrm{keV}$ line in a very simple way, introduces a coupling between the Higgs boson and a SM neutrinos of the form $\tilde{\lambda} \bar{\psi} H \ell$, where for simplicity we have suppressed generation indices, thus identifying DM with a sterile right-handed neutrino. In this case the radiative decay of DM is achieved, irrespectively of couplings and mass of the scalar field, through loops involving charged leptons and the $W$ boson, as shown in Figure 5.6.

The decay rate of the DM is given by [234]:

$$
\Gamma(\psi \rightarrow \nu \gamma)=\frac{9 \alpha G_{F}^{2} m_{\psi}^{5}}{2564 \pi^{4}} \sin ^{2} 2 \Theta
$$

where $\Theta=\frac{\tilde{\lambda} v}{m_{\psi}}$. The required value of the DM lifetime is obtained for $\sin ^{2} 2 \Theta=2-20 \times 10^{-11}$ which corresponds to $\tilde{\lambda} \simeq 10^{-13}$.

Although the scalar field is not responsible for the radiative decay of DM, its presence is anyway relevant for the DM production. As already shown for instance in [226, 235-238], the 
decay of an extra field provides a simple and economical mechanism for the production of sterile neutrinos with a low value of mixing angle with active neutrinos, which is compatible with the DM Indirect Detection. In fact, first, the conventional, non-resonant, production through oscillations from active neutrinos, known as the Dodelson-Widrow mechanism [239], can provide no more than $1 \%$ of the DM relic density for the values of the parameters accounting for the present $X$-ray signal, and second, its resonant enhancement [240] requires the presence of a very large lepton asymmetry at low temperatures, which is not trivial to achieve in realistic scenarios [241]. In Figure 5.7 the Dodelson-Widrow mechanism (red band) along with the X-ray constraints coming from Chandra and XMM-Newton observations of M31 (light blue region) and Suzaku observations of Ursa Minor (green region) are displayed. In addition, the red dot represents the detection of the $3.55 \mathrm{keV}$ line (assuming that such a line is from sterile neutrino and that all dark matter is in sterile neutrino) from the stacked XMM-Newton MOS observations of galaxy clusters found in [179].

The LHC phenomenology depends on the size of eventual couplings between the scalar field $\Sigma_{f}$ with only SM fields. These kinds of couplings are completely uncorrelated to DM phenomenology and are constrained only by the assumption that they do not contribute substantially to the DM decay nor allow for fast proton decay. The most interesting case is therefore when their value is suppressed and, as could be argued for example by a common generation mechanisms, is comparable with the one of the other couplings either $\tilde{\lambda}$ or $\lambda$ fixed as in Equation (5.5). In such a case we would expect an LHC-metastable $\Sigma$ field, whose prospects of detection have extensively discussed in [242]. Such a scenario is shown in Figure 5.3 and 5.5 in terms of the brown line and the green band respectively.

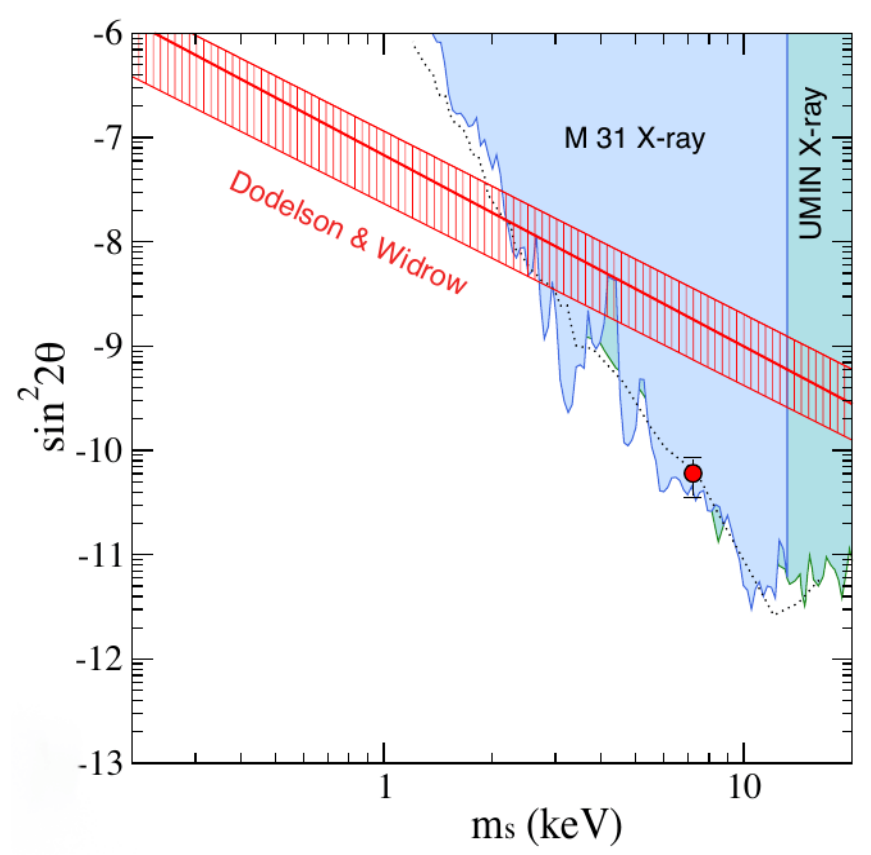

FIGURE 5.7: Constraints on the sterile neutrino model from the literature given by [179]. The full-sample MOS line detection is shown by red symbol. X-ray constraints from Chandra and XMM-Newton observations of M31 and Suzaku observations of Ursa Minor are painted light blue and green, respectively. The red band marked "Dodenson 83 Widrow" is for the part of the parameter space where Dodelson-Widrow mechanism takes place. 
Before stating our conclusions we will mention possible additional constraints coming from structure formation (these are actually customarily discussed in sterile neutrino scenarios but most of the arguments can be straightforwardly extended to generic particle physics frameworks, including the one discussed in this work). Indeed DM with mass at the $\mathrm{keV}$ scale is conventionally considered as warm dark matter. The viability of warm DM candidates, as opposed to cold dark matter, is currently tested through rather different strategies that typically lead to lower limits on the DM mass. Interestingly the degree of "warmness" of the DM depends on its momentum distribution function which in turn is determined by its production mechanism. It is then possible, potentially, to exploit the bounds from structure formation to constrain the particular freeze-in production mechanism, and exclude scenarios that produce too warm DM.

Although several studies have determined the DM distribution function for particles produced through freeze-in $[235,243]$ and, more in general, from the decay of a scalar field with different cosmological histories [244,245], no quantitative analysis of structure formation for this kind of scenarios have been performed yet. As shown e.g. in [235, 243], for the same DM mass, DM produced by freeze-in has a lower average momentum with respect to a thermal relics or a sterile neutrino arising from the DW mechanism. A lower average momentum can be interpreted as a "colder" DM momentum distribution; as consequence it is customarily stated that DM production from decay is more weakly constrained by structure formation bounds. On the other hand a very recent study [245] has studied this approximation envisaging instead a tension between structure formation and the freeze-in paradigm for $\mathrm{keV}$ scale DM. Even in this last case no definitive conclusion could be derived because of the several approximations adopted. Therefore, a definitive word on the viability of keV DM produced by freeze-in requires dedicated numerical simulations.

\subsection{Conclusions}

We have here considered the possibility of reproducing the $3.55 \mathrm{keV}$ X-ray line signal in few simple decaying DM scenarios. In the minimal realization, namely the extension of the SM with a DM Majorana fermion and a single scalar field at the $\mathrm{TeV}$ scale, it is possible to produce the right abundance of DM and obtain the correct DM lifetime for reasonable values of the couplings $\lambda$ and $\lambda^{\prime}$. The model then predicts prompt decays of the colored $\Sigma_{f}$ scalar at LHC though the larger coupling $\lambda^{\prime}$ in only Standard Model states. Even if the second decay channel of $\Sigma_{f}$ is too suppressed to be observable at LHC, the scenario can be tested and even excluded, if no prompt decays are observed, up to masses of the colored scalar field of about $1400 \mathrm{GeV}$. Moreover in case of a detection, the parameters of the model can be restricted by a combination of the Indirect Detection and LHC measurements and the assumption of freeze-in production for DM can be in principle tested.

We tried to see if it is possible to lower the $\lambda^{\prime}$ coupling and enhance the possibility to measure both decay channels at LHC. Unfortunately this turned out to be not so simple to achieve. Enlarging the Dark Matter and $\Sigma_{f}$ sector to have a top particle in the loop does allow for a wider range of possible $\lambda^{\prime}$ couplings, but does not modify strongly the hierarchy between $\lambda^{\prime}$ and $\lambda$. In this extended model DM decays into a photon and an extremely light SM singlet, which can affect the number of cosmological relativistic degrees of freedom $N_{\text {eff }}$ and in an optimistic case perhaps be detected in the CMB. In this case the couplings between the $\Sigma_{f}$ fields and purely SM fields are not fixed by the DM lifetime and the phenomenology strongly depends on their values. Indeed, if they are comparable to the coupling of the scalar with the 
new singlet fermion $\chi$, the scalar $\Sigma$ could decay promptly at LHC in the two channels. Then it may be possible to contemporary observe prompt decays into top quark and missing energy as well as decays into only SM states and determine again most of the parameters of the model. On the other hand, if the couplings of the $\Sigma_{f}$ fields with only SM fields are of the order of the freeze-in coupling $\lambda$, the scenario will be difficult to disentangle at LHC, since the singlet fermion $\chi$ could appear as a possible DM candidate.

We have finally considered the case in which the DM has the additional coupling to the Higgs field and a SM neutrino, similarly to a sterile neutrino. The DM lifetime is determined only by the new mixing, but the coupling of DM with the SM-charged field $\Sigma_{f}$ can help in obtaining the right DM abundance. In this last case, if also the couplings of $\Sigma_{f}$ to SM fields are of similar size to $\lambda$, a detection of both $\Sigma_{f}$ decays through displaced vertices could be possible at LHC and allow to disentangle this scenario from a pure sterile neutrino. 



\section{Final Conclusions}

The identification of nature of DM is still one of the most important puzzles in modern astrophysics and particle physics. Even though the conventional paradigms rely on stable DM particles, no a-prior argument exists against decaying DM candidates as long as their lifetime is much longer that the age of the Universe. The longevity of DM demands that the couplings of the DM with ordinary matter are too suppressed to allow direct detection of DM in experiments like e.g. XENON, LUX. On the contrary, there are rather promising prospects on indirect detection (ID), in cosmic rays, of the DM decays occurring at present times. Already strong limits on the DM lifetime have been set on a broad variety of decay products by observations like those performed by Fermi, AMS and XMM/CHANDRA. On the other hand, high-energy colliders, such as the CERN Large Hadron Collider, are ideal machines for producing and eventually detecting DM. Experiments in upcoming colliders, such as the ILC and CLIC, are expected to further constraint DM models, if they are materialized in nature, and subsequently make a key step in understanding DM.

In the light of this exciting background we have studied in this thesis basically two different models of decaying DM at colliders and two simple extensions of the second model which are allowed for new couplings and fields. Both models present distinct signals at colliders and indirect detection (ID) and those signals, if detected, can allow to distinguish the two models and in the most optimistic case determine the couplings between DM and the SM sector. Moreover, the couplings in both models are very suppressed either by the Planck scale suppression, or by construction in the second case. These models therefore tend to give long lifetimes to one state that is charged under the SM gauge group, and so can be produced at LHC or ILC/CLIC. Although such a charged state is not DM directly, it can give information on the couplings of DM with the SM particles if it is the lightest "mediator". Interestingly, these two models, one very complex and based on an extended symmetry, the other very simple and with a minimal particle and coupling content, can give rise to very similar signatures at collider and in indirect detection.

In the first model we have considered the case of stop NLSP with gravitino LSP and DM, under the assumption that stop is the only state that is accessible at LHC, and then shown that it is possible to probe the model up to stop masses of $1300 \mathrm{GeV}$ and $2100 \mathrm{GeV}$ for the integrated luminosity of $L=25 \mathrm{fb}^{-1}$ and $3000 \mathrm{fb}^{-1}$, respectively. In order to obtain these results we have used both a numerical procedure, based on MadGraph 5 and Python to estimate the stop production and its exponential decay respectively, and a simple analytical procedure, based instead on the well-known exponential decay formula and the MadGraph production crosssection. We have then reformulated the obtained stop LHC reach in the parameter space of the two models with gravitino DM, either RPC and RPV, i.e. stop lifetime-stop mass and RP breaking parameter-gravitino mass respectively. Thus, in the RPC scenario we have obtained metastable tracks for high reheating temperature whereas displaced vertices for lower reheating 
temperatures and smaller gravitino masses. In the RPV scenario on the other hand we have obtained a small viable region for small stop masses between the potential metastable particle excluded region and indirect detection one. Finally, we have seen that in the case of detectable displaced vertices the RPC and RPV scenarios can be distinguished from one another by their different kinematics.

In the second model, we have changed DM search strategy because of the small number of parameters coming into play in this model. In fact, we have not only considered the reach, but we have also tried to see if it is possible to determine all the parameters of the model by combining all the available data. Interestingly, there is a region of the parameter space ("double" detection region) in which, although most of the information can be extracted from LHC or CLIC, the coupling $\lambda$ remains however hard to obtain. The goal of this search is indeed to determine the model parameters and not the double detection region by itself.

In this second model we have also accommodated both keV DM with the right decay rate and FIMP production, but then in the minimal case the scalar particle has to decay promptly at LHC, making more difficult the measure of the lifetime, and so of the couplings. In the extended models, on the other hand, displaced vertices can still be a chance, but only in particular cases.

While we wait for the LHC data coming from the present run, we point out that an experimental combined analysis of metastable tracks and displaced vertices including detector simulations could refine our analyses, and so modify our picture. Moreover, in case nothing is found at LHC up to very large mass scales, we have no information about the DM production mechanism since Freeze-in mechanism can even work with much larger $x\left(=m_{D M} / m_{\Sigma}\right)$ as long as the out-of-equilibrium is satisfied. On the other hand, at larger scalar masses, the SuperWIMP should/could take over because of the larger scalar relic abundance $\Omega_{\Sigma} h^{2}$, and the $\Sigma$ mass where this happens could be actually estimated once the charge of $\Sigma$ is fixed. 


\section{Appendix A}

\section{Python code for exponential decay}

In this appendix the python routine for the stop exponential decay studied in Chapter 3 is displayed. Particularly, we have used this code to include in the MG-output (*.lhe file) the distance from the interaction point that each stop travels before decaying. In order to make the code clearer to the readers, first we add some comments, signaled by the symbol \#, to the lines of code which are more ambiguous. Second we show below the code in Figure A.1 a modified MG-output-event, obtained for $m_{\tilde{t}}=800 \mathrm{GeV}$ and $\Gamma_{\tilde{t}}=10^{-10} \mathrm{GeV}$.

Note that python has added the 11th column "distance" of the table which elements are expressed in millimeters. The elements of the columns named " $p_{x}$ ", " $p_{y}$ ", " $p_{z}$ ", " $p_{0}$ " and "mass" are instead expressed in $\mathrm{GeV}$. Due to time restrictions we do not explain here all the entries of this table. However, for some more details on the technical aspects of such a MG-output, the reader is encouraged to consult the notes written by Flip Tanedo [246].

The exponential distribution in python is given by the function "random.expovariate (lambd)" where lambd is 1.0 divided by the desired mean. The returned values range from 0 to positive infinity if lambd is positive, and from negative infinity to 0 if lambd is negative. Specifically, according to the python webpage ${ }^{2}$, "random.expovariate $(\lambda)$ " is capable to give the decay length of each decaying stop particle by using the exponential distribution:

$$
f(x, \lambda)=\lambda \exp \{-\lambda x\}, \quad \lambda=\frac{\Gamma}{\gamma \beta},
$$

with the decay rate $\Gamma$, the Lorentz factor $\gamma$ and the beta factor $\beta$ (see Section \# for their definitions) computed by the Madgraph output, according to the identity:

$$
\lambda=\frac{\Gamma}{\gamma \beta}=\Gamma \sqrt{1-\frac{p^{2}}{E^{2}}} \frac{E}{|p|}=\Gamma \sqrt{1-\frac{\left(p_{x}^{2}+p_{y}^{2}+p_{z}^{2}\right)^{2}}{p_{0}^{2}}} \frac{p_{0}}{\sqrt{p_{x}^{2}+p_{y}^{2}+p_{z}^{2}}} .
$$

This python code has also been used to include in the MG-output the decay lengths of the new scalars $\Sigma_{d}, \Sigma_{\ell}$ and $\Sigma_{e}$, taken into account in Chapter 4 . In more detail, since these new scalars have the same quantum numbers of a right handed bottom quark singlet, an $\mathrm{EW} \mathrm{SU}(2)_{L}$ lepton doublet and a right handed lepton singlet, respectively, the analysis of their decay can be reduced to the analysis of the SUSY particles with the same quantum numbers.

\footnotetext{
${ }^{1}$ The first column "pid" is counted as the column number zero.

${ }^{2}$ https://docs. python.org/2/library/random.html?highlight=random.expovariate\#random.expovariate.
} 


$\left.\begin{array}{cccccccccccccc}\text { pid } & \text { in } & \text { mother1 } & \text { mother2 } & \text { color } 1 & \text { color } 2 & \text { px } & \text { py } & \text { pz } & \text { p0 } & \text { mass } & \text { distance } & \text { hel } \\ \text { uBar } & \text { in } & 0 & 0 & 0 & 501 & 0 . & 0 . & 576.918 & 576.918 & 0 . & 0 . & -1 . \\ \mathrm{u} & \text { in } & 0 & 0 & 502 & 0 & 0 . & 0 . & -1681.09 & 1681.09 & 0 . & 0 . & 1 . \\ \tilde{t}_{1} & \text { decayed } & 1 & 2 & 502 & 0 & 237.699 & 484.863 & -776.314 & 1238.65 & 800 . & 0.000185211 & 0 . \\ \left(\tilde{t}_{1}\right)^{*} & \text { decayed } & 1 & 2 & 0 & 501 & -237.699 & -484.863 & -327.855 & 1019.35 & 800 . & 0.000554574 & 0 . \\ \mathrm{b} & \text { out } & 3 & 3 & 502 & 0 & 503.895 & 242.913 & -279.689 & 625.433 & 4.88992 & 0 . & 1 . \\ \mu^{+} & \text {out } & 3 & 3 & 0 & 0 & -266.195 & 241.95 & -496.626 & 613.219 & 0 . & 0 . & 1 . \\ \text { bBar } & \text { out } & 4 & 4 & 0 & 501 & 112.019 & -452.578 & 88.5704 & 474.598 & 4.88992 & 0 . & -1 . \\ \text { particle [15] } & \text { out } & 4 & 4 & 0 & 0 & -349.718 & -32.2848 & -416.425 & 544.755 & 1.777 & 0 . & -1 .\end{array}\right)$

Figure A.1: 1 MG-output-event for $m_{\tilde{t}}=800 \mathrm{GeV}, \Gamma_{\tilde{t}}=10^{-10} \mathrm{GeV}$ after the python implementation.

\section{Code}

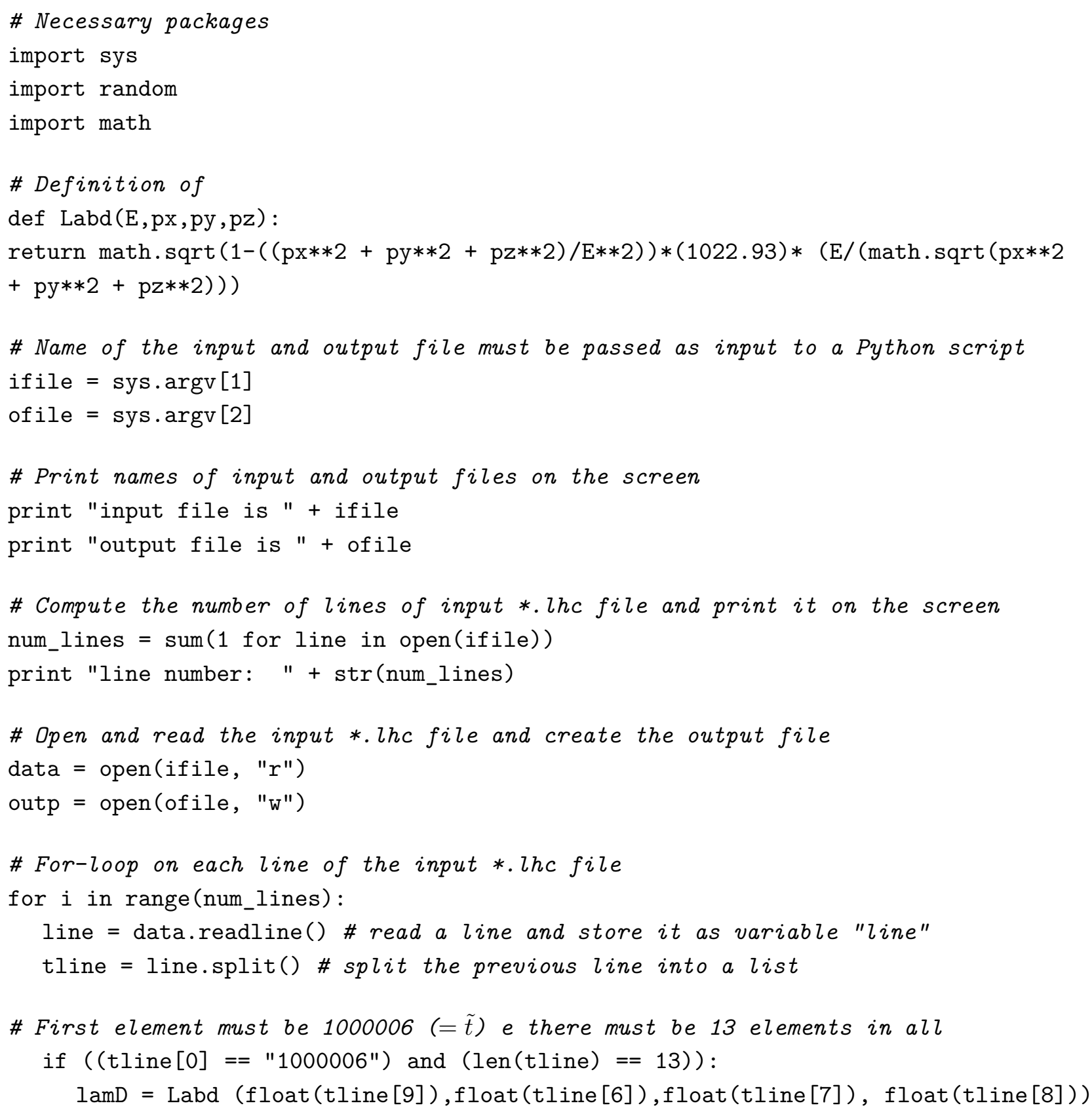




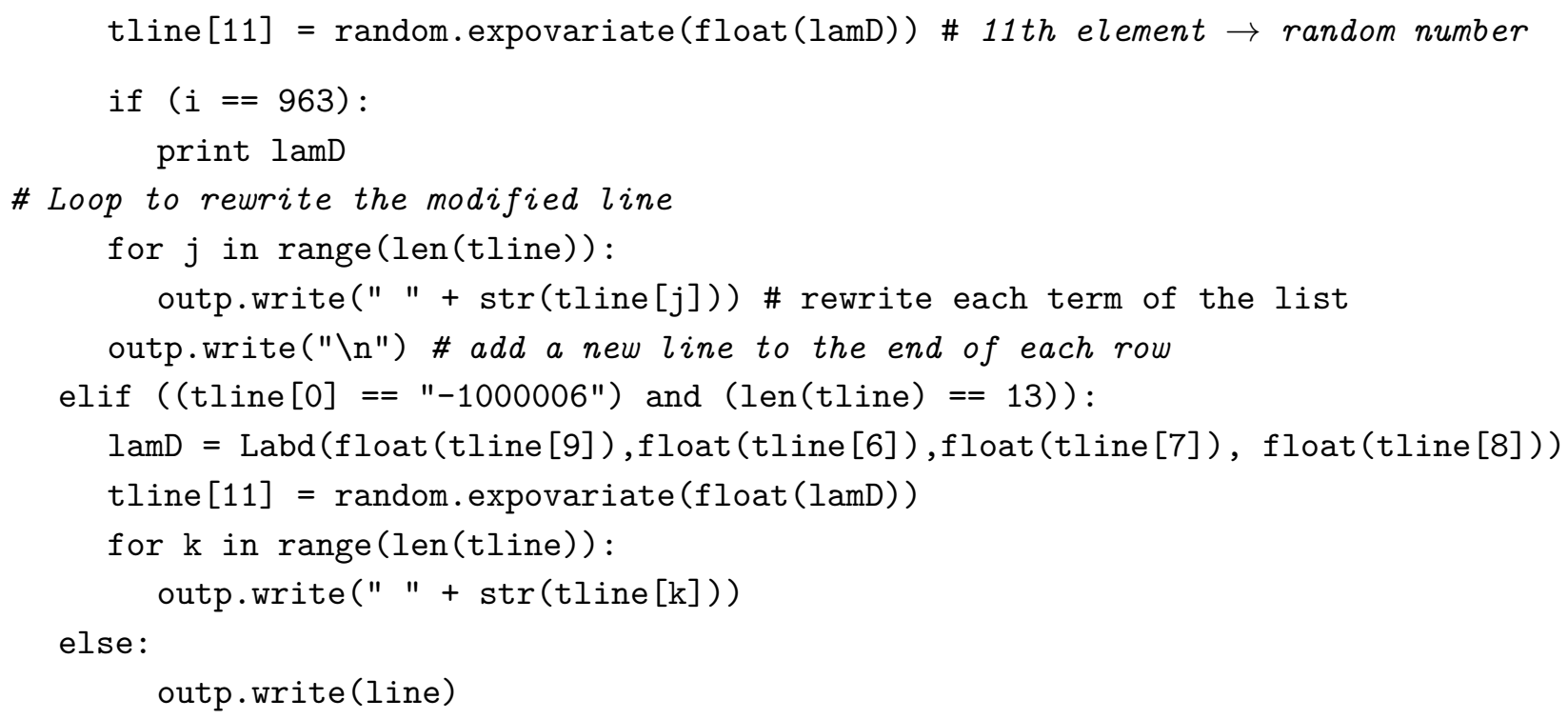





\section{Appendix B}

\section{Mathematica code}

In this appendix we exhibit the mathematica code we have used in order to analyse the pythonoutput and eventually extract the information which is relevant to obtain the distribution of the stop displayed vertices and compute how many displaced vertices appear inside Pixel and Tracker and outside CMS detector. In other words, this code allow to achieve the Pixel reach, the Tracker reach and the reach of the metastable particle, namely most of the plots of Chapter 3 and 4 .

This routine is the content of the file "MadEvent_analysis.nb". It is based on the Chameleon package for the LHC Olympics developed by Philip Schuster, Jesse Thaler, and Natalia Toro [247]. This package was modified by Maxim Perelstein and Andreas Weiler in 2008-2009 and later by Flip Tanedo in 2011 [246]. We have slightly modified Tanedo's file in order to implement our displaced vertex + metastable particle analysis. Eventually we ended up with a set of definitions for the functions to input an LHE file and analyze its events.

Note that although the following code has been written to investigate the decaying stop, it can even be used for any decaying particle if we make the appropriate modifications. In fact, we have exploited this routine to study the decay of the three scalars $\Sigma_{d}, \Sigma_{\ell}$ and $\Sigma_{e}$ of Chapter 4 once we have included their decay lengths in the Madgraph-output via the python code of Appendix A. Similarly to the python code, such an investigation occurs through the investigations of the SUSY particles having the same quantum numbers of the scalars.

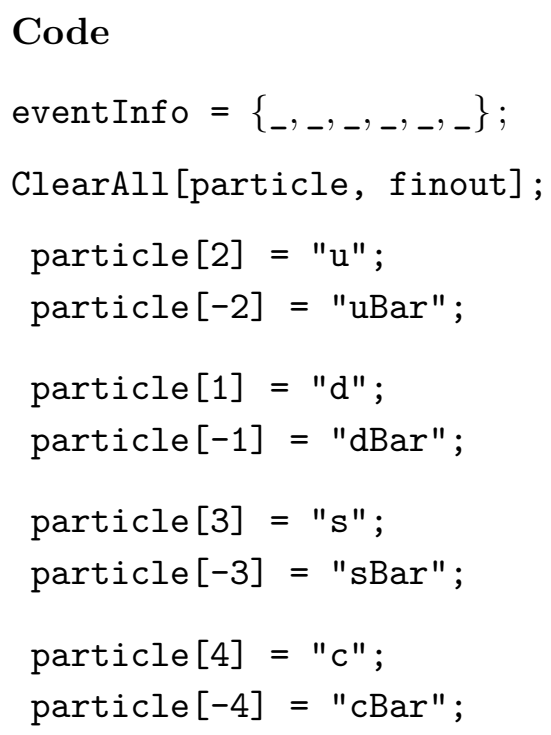




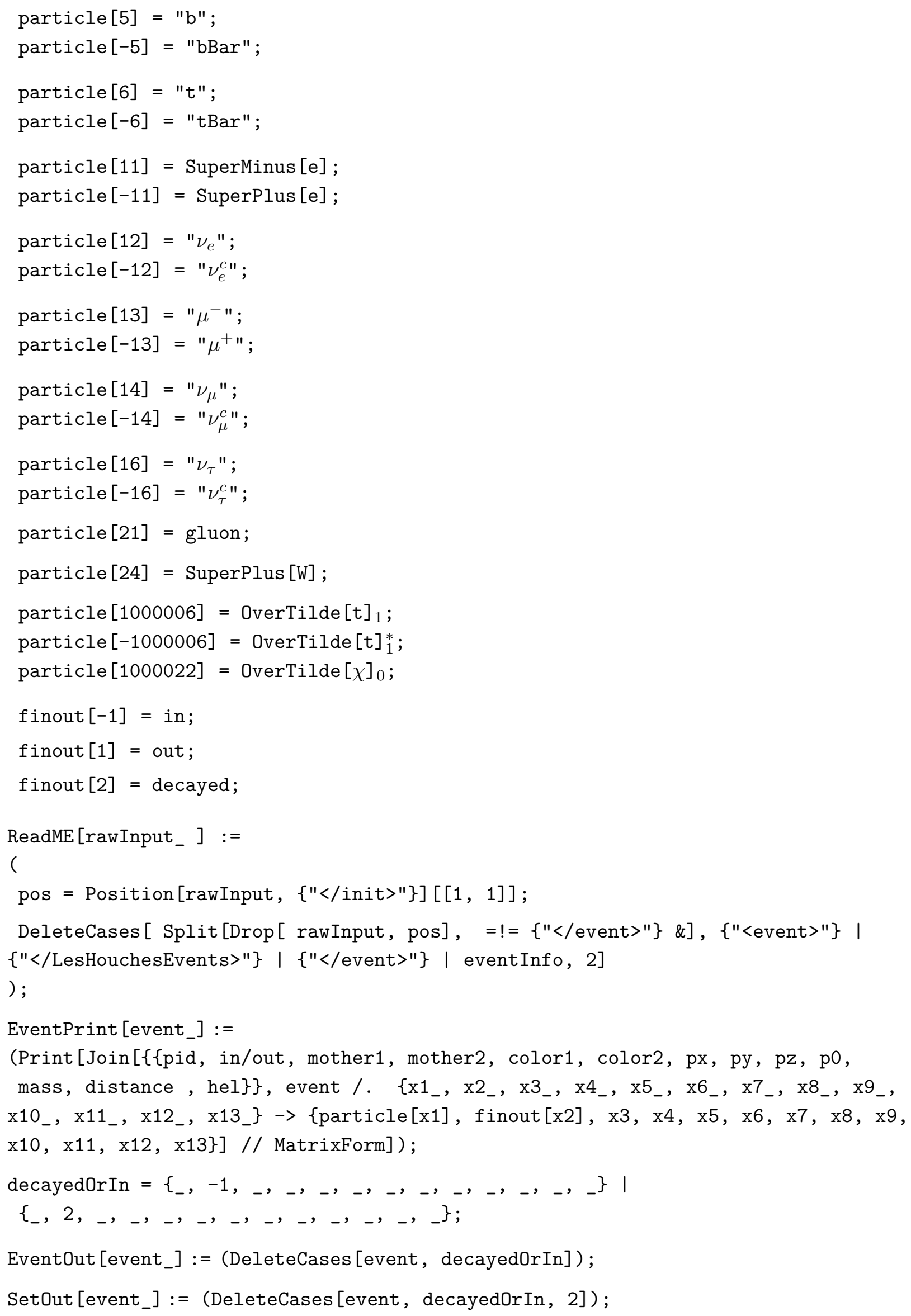

EventOut [event_] := (DeleteCases [event, decayedOrIn]);

SetOut [event_] := (DeleteCases [event, decayedOrIn, 2]); 
EffMass [event_] := Plus @e Map[ptOf, event, \{1\}];

pT[event_] := Map[ptOf, event, \{1\}] // Flatten;

eta[event_] := Map[etaOf, event, \{1\}] // Flatten;

theta[event_] := Map[thetaOf, event, \{1\}] // Flatten;

threeVector[event_] := Map[ThreeVectorFrom, event, $\{1\}]$;

piy[event_] := Map[Py, event, \{1\}] // Flatten;

piz [event_] := Map $[\mathrm{Pz}$, event, \{1\}] // Flatten;

pix[event_] := Map [Px, event, \{1\}] // Flatten;

MissingEnergy [event_] := Plus @@ Map[enOf, event, \{1\}];

betA[event_] := Map[betaOf, event, \{1\}] // Flatten;

gammA[event_] := Map [gammaOf, event, $\{1\}] / /$ Flatten;

dist[event_] := Map[dist0f, event, \{1\}] // Flatten;

directRx[event_] := Map[directionRxOf, event, \{1\}] // Flatten;

directRy[event_] := Map[directionRyOf, event, \{1\}] // Flatten;

directRz[event_] := Map[directionRzOf, event, \{1\}] // Flatten;

directRTVector[event_] := Map[directionRTVectorOf, event, \{1\}] // Flatten;

Rsqrtx2y2[event_] := Map[Rsqrtx2y20f, event, \{1\}] // Flatten;

$\operatorname{ptOf}\left[\left\{_{-},{ }_{-},{ }_{-},{ }_{-},{ }_{-},{ }_{-}, \mathrm{px}_{-}, \mathrm{py}_{-},{ }_{-}{ }_{-}\right\}\right]:=\operatorname{Sqrt}\left[\mathrm{px}^{2}+\mathrm{py}^{2}\right]$;

enOf $\left[\left\{_{-},,_{-},,_{-},,_{-},,_{-},{ }_{-},{ }_{-}, \mathrm{En}_{-},{ }_{--}\right\}\right]:=\mathrm{En}$;

thetaof $\left[\left\{_{-},{ }_{-},{ }_{-},{ }_{-},{ }_{-}, \mathrm{H}_{-}, \mathrm{px}_{-}, \mathrm{py}_{-}, \mathrm{pz}_{-}, \ldots_{-}\right\}_{-}\right]:=\operatorname{ArcCos}\left[\mathrm{pz} / \operatorname{Sqrt}\left[\mathrm{px}^{2}+\mathrm{py}^{2}+\mathrm{pz}^{2}\right]\right]$;

$\left.\operatorname{etaOf}\left[\left\{_{-},{ }_{-},,_{-},,_{-},{ }_{-}, \mathrm{px}_{-}, \mathrm{py}_{-}, \mathrm{pz}_{-},{ }_{-}{ }_{-}\right\}\right]\right\}:=-\log [\mathrm{Abs}[\operatorname{Tan}[$

ArcCos $\left.\left.\left.\left[\mathrm{pz} / \operatorname{Sqrt}\left[\mathrm{px}^{2}+\mathrm{py}^{2}+\mathrm{pz}^{2}\right]\right] / 2\right]\right]\right]$;

FourVectorFrom $\left[\left\{_{-},{ }_{-},{ }_{-},{ }_{-},{ }_{-}, \mathrm{px}_{-}, \mathrm{py}_{-}, \mathrm{pz}_{-}, \mathrm{En}_{-},{ }_{--}\right\}\right]:=\{\mathrm{En}, \mathrm{px}, \mathrm{py}, \mathrm{pz}\}$;

FourLength $\left[\left\{\mathrm{pe}_{-}, \mathrm{pz}_{-}, \mathrm{px} \mathrm{x}_{-}, \mathrm{py} \mathrm{p}_{-}\right\}\right]:=\operatorname{Sqrt}\left[\operatorname{Max}\left[\mathrm{pe}^{2}-\mathrm{pz}^{2}-\mathrm{px}^{2}-\mathrm{py}^{2}, 0.0\right]\right] ;$

ThreeVectorfrom $\left[\left\{_{-},{ }_{-},{ }_{-},{ }_{-},{ }_{-},{ }_{-}, \mathrm{px}_{-}, \mathrm{py}_{-}, \mathrm{pz}_{-},{ }_{--}\right\}\right]:=\{\mathrm{px}, \mathrm{py}, \mathrm{pz}\}$;

Py $\left.\left[\left\{_{-},{ }_{-},,_{-},,_{-},,_{-}, \mathrm{py}_{-},{ }_{-},{ }_{-}\right\}\right]\right]:=\mathrm{py}$;

$\operatorname{Px}\left[\left\{_{-},{ }_{-},,_{-},,_{-}, \mathrm{px}_{-},,_{-},{ }_{-}{ }_{--}\right\}\right]:=\mathrm{px}$;

$\mathrm{Pz}\left[\left\{_{-},{ }_{-},,_{-},,_{-},,_{-},{ }_{-}, \mathrm{pz}_{-},{ }_{-}{ }_{-}\right\}\right]:=\mathrm{pz}$;

$\operatorname{betaOf}\left[\left\{_{-},{ }_{-},,_{-},,_{-},{ }_{-}, \mathrm{px}_{-}, \mathrm{py}_{-}, \mathrm{pz}_{-}, \mathrm{En}_{-},{ }_{--}\right\}\right]:=\operatorname{Sqrt}\left[\mathrm{px}{ }^{2}+\mathrm{py}^{2}+\mathrm{pz}^{2}\right] / \mathrm{En}$;

gammaOf $\left[\left\{_{-},,_{-},,_{-},,_{-}, \mathrm{px}_{-}, \mathrm{py}_{-}, \mathrm{pz}_{-}, \mathrm{En}_{-},{ }_{--}\right\}\right]:=$

$\left(1-\left(\left(\mathrm{px}^{2}+\mathrm{py}^{2}+\mathrm{pz}^{2}\right) / \mathrm{En}^{2}\right)\right)^{(-1 / 2)}$;

$\operatorname{dist0f}\left[\left\{_{-},{ }_{-},{ }_{-},,_{-},{ }_{-},,_{-},{ }_{-},{ }_{-},{ }_{-}, \mathrm{dsr} r_{-},{ }_{-}\right\}_{-}\right]:=\mathrm{dsr}$;

directionRxOf $\left[\left\{_{-},{ }_{-},{ }_{-},{ }_{-},{ }_{-}, \mathrm{px}_{-}, \mathrm{py}_{-}, \mathrm{pz}_{-},,_{-}, \mathrm{d}_{-}, \mathrm{dsr} \mathrm{r}_{-}\right\}\right]:=$

$\mathrm{px} * \mathrm{dsr} / \operatorname{Sqrt}\left[\mathrm{px}^{2}+\mathrm{py}^{2}+\mathrm{pz}^{2}\right]$;

directionRyOf $\left[\left\{_{-},{ }_{-},{ }_{-},{ }_{-},{ }_{-}, \mathrm{px}_{-}, \mathrm{py}_{-}, \mathrm{pz}_{-},{ }_{-}, \mathrm{H}_{-}, \mathrm{dsr} \mathrm{r}_{-},{ }_{--}\right\}\right]:=$

$\mathrm{py} * \mathrm{dsr} / \operatorname{Sqrt}\left[\mathrm{px}^{2}+\mathrm{py}^{2}+\mathrm{pz}^{2}\right]$;

directionRzOf $\left[\left\{_{-},{ }_{-},{ }_{-},{ }_{-},{ }_{-}, \mathrm{px}_{-}, \mathrm{py}_{-}, \mathrm{pz}_{-},{ }_{-}, \mathrm{H}_{-}, \mathrm{dsr} \mathrm{r}_{-},{ }_{--}\right\}\right]:=$ $\mathrm{pz} * \mathrm{dsr} /$ Sqrt $\left[\mathrm{px}^{2}+\mathrm{py}^{2}+\mathrm{pz}^{2}\right]$;

directionRTVectorOf $\left[\left\{_{-},{ }_{-},{ }_{-},,_{-},{ }_{-}, \mathrm{px}_{-}, \mathrm{py}_{-}, \mathrm{pz}_{-},,_{-}, \mathrm{dsr}_{-}, \mathrm{-}_{--}\right\}\right]:=$ 


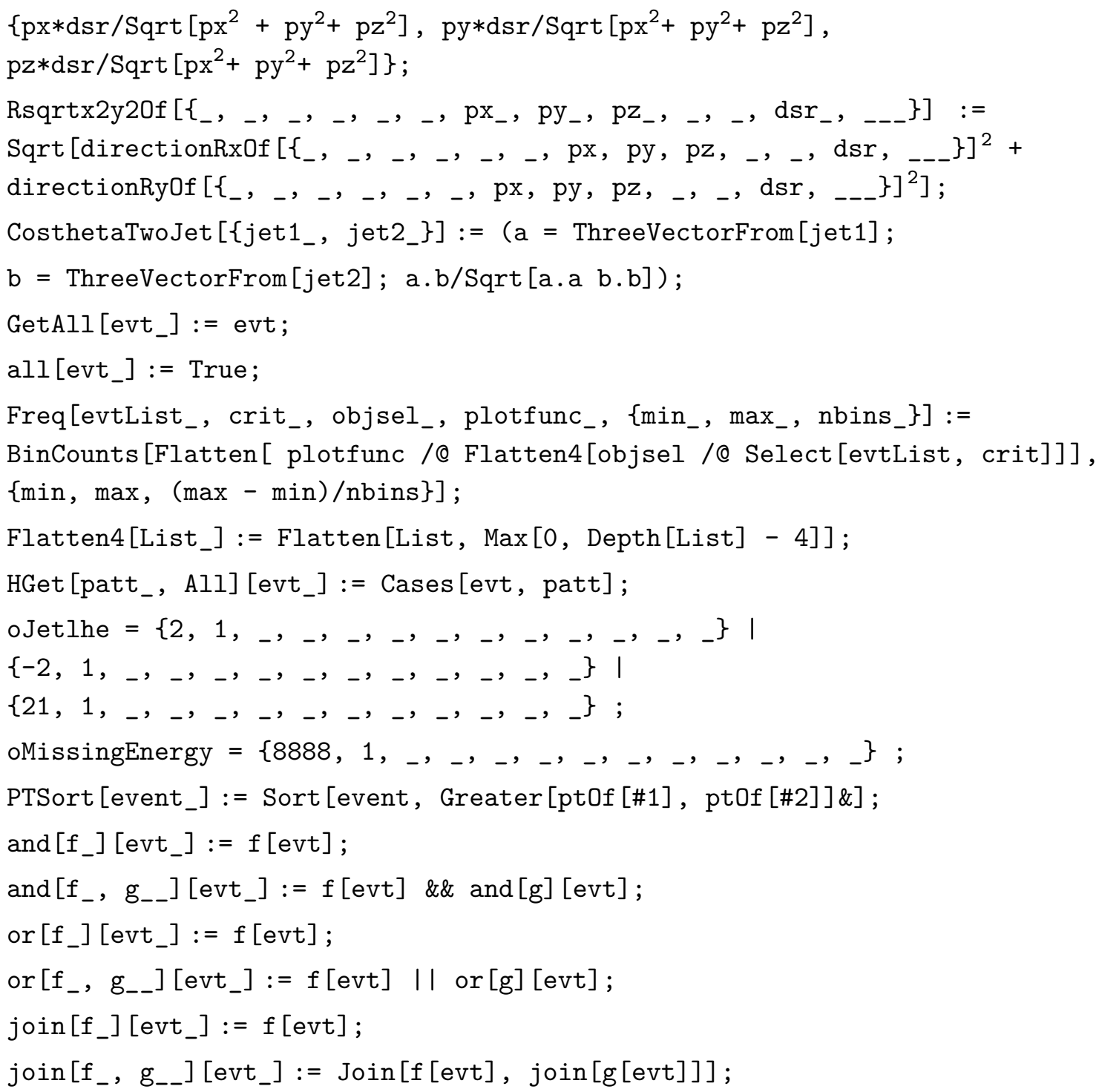




\section{Appendix C}

\section{FeynRules code}

At the end of Chapter 3 we have discussed how to distinguish the RPC stop decay into gravitino $\psi_{3 / 2}$ and top $t$ from the RPV stop decay into vottom $b$ and anti-lepton $\ell^{+}$if they occur inside the detector parts of CMS under consideration (i.e. Pixel and Tracker). In particular, we have compared the 2-body RPC stop decay: $\tilde{t} \rightarrow b \ell^{+}$, with the 4-body RPV stop decay chain: $\tilde{t} \rightarrow t \psi_{3 / 2} \rightarrow W^{+} b \psi_{3 / 2} \rightarrow b \ell^{+} \nu_{\ell} \psi_{3 / 2}$, which clearly have the same final visible particles: $b, \ell^{+}$.

The different particle kinematics of the two chains and the missing energy of the RPV decay allowed to distinguish these two processes from one another. Specifically, we have taken into consideration the Madgraph distributions of the antilepton transverse momentum $\left(P_{\ell T}\right)$, the transverse mass of the pair $\ell^{+}-b\left(M_{T}\right)$ and, finally, the angle between $b$ and $\ell^{+}\left(\theta_{\ell b}\right)^{1}$.

Unlike the RPC stop decay, which was implemented in the directory "models" of MadGraph by Matt Reece releasing then the code at http://users.physics.harvard.edu/mreece/stopnlsp/, the RPV stop decay (precisely the RPV vertex) was not implemented in MadGraph during the completion of this work. Thus we had to include it on our own to the code that defines the model "mssm" and then run the package FeynRules [163] in order to generate the model which can be used by MadGraph. Due to time restrictions we do not explain here how to run FeynRules, we instead show the few additional lines of code to add to the FeynRules file "mssm.fr", which define the MSSM model ${ }^{2}$. By following this procedure, we have obtained the FeynRules file "RPVstopdecay.fr" that allowed to create the Feynman rules for the RPV vertex under consideration, and so the model "RPVstopdecay" suitable to MadGraph. Notice that the package Mathematica was exploited to obtain the latter model.

The above-mentioned FeynRules file "mssm.fr" consists of many different sections, all needed to generate the model "mssm" appropriate for MadGraph. Below we display the code lines to add to the section "Superpotential", which is contained in the bigger section "Lagrangian".

For the purposes of completeness, we remark that, even though the implementation by Matt Reece has been written for a goldstino instead of a gravitino, it remains valid for our scenario since gravitino is light enough to be considered dominated by its own $1 / 2$-spin component.

\footnotetext{
${ }^{1}$ For definitions see Equation (3.25), (3.26) and (3.27).

${ }^{2}$ See Section 1.1 for a basic introduction of this model.
} 


\section{Code}

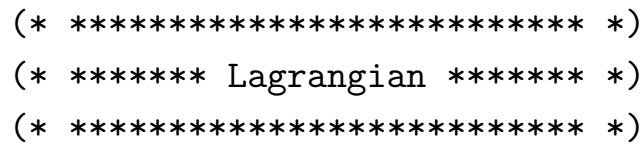

(* Superpotential $*$ )

LLQD [ff4,ff5,ff3] Conjugate[CKM[ff2,ff5]] Conjugate[PMNS[ff1,ff4]] DR[ff3,cc1] (LL[1,ff1] QL $[2, f f 2, \mathrm{cc} 1]-\mathrm{LL}[2, \mathrm{ff} 1] \mathrm{QL}[1, \mathrm{ff} 2, \mathrm{cc} 1])$ 


\section{Acknowledgements}

First of all I would like to thank my supervisor Prof. Dr. Laura Covi who suggested this work. I am grateful for her good advice, constant support and valuable comments.

I further want to thank my other collaborator Dr. Giorgio Arcadi, for his commitment and for many hours of discussion.

Last but not least, I would like to thank my family and all my friends both in Göttingen and in Castiglione di Ravenna. 



\section{Bibliography}

[1] S. P. Martin, A Supersymmetry primer, hep-ph/9709356.

[2] B. de Wit, Supergravity, hep-th/0212245.

[3] M. E. Peskin, Supersymmetry in Elementary Particle Physics, arXiv:0801.1928.

[4] K. A. Olive, Introduction to Supersymmetry: Astrophysical and Phenomenological Constraints, hep-ph/9911307.

[5] M. Dress, An Introduction to Supersymmetry, hep-ph/9611409.

[6] P. M. R. Binétruy, Supersymmetry: Theory, Experiment, and Cosmology. Oxford Graduate Texts, 2012.

[7] J. Wess and J. Bagger, Supersymmetry and Supergravity. Princeton University Press, 1992.

[8] G. Arcadi and L. Covi, Minimal Decaying Dark Matter and the LHC, JCAP 1308 (2013) 005, [arXiv: 1305.6587].

[9] CMS Collaboration, Observation of a new boson at a mass of $125 \mathrm{GeV}$ with the CMS experiment at the LHC, Phys. Lett. B716 (July, 2012) 30, [arXiv:1207.7235].

[10] ATLAS Collaboration, Observation of a new particle in the search for the Standard Model Higgs boson with the ATLAS detector at the LHC, Phys. Lett. $\mathbf{B 7 1 6}$ (July, 2012) 1-29, [arXiv: 1207.7214].

[11] R. Haag, J. T. Lopuszanski, and M. Sohnius, All Possible Generators of Supersymmetries of the S Matrix, Nucl. Phys. B88 (1975) 257.

[12] S. Coleman and J. Mandula, All Possible Symmetries of the S Matrix, Nucl. Phys. 159 (1967) 1251-1256.

[13] J. S. Dimopoulos and H. Georgi, Softly broken supersymmetry and su(5), Nucl. Phys. B193 (1981) 150.

[14] L. J. Hall, D. Pinner, and J. T. Ruderman, A natural susy higgs near 125 gev, JHEP 1204 (2012) 131, [arXiv:1112.2703]. 
[15] S. Heinemeyer, O. Stal, and G. Weiglein, Interpreting the lhc higgs search results in the mssm, Phys. Lett. B710 (2012) 201, [arXiv:1112.3026].

[16] A. Arbey, M. Battaglia, A. Djouadi, F. Mahmoudi, and J. Quevillon, Implications of a 125 gev higgs for supersymmetric models, Phys. Lett. B708 (2012) 162, [arXiv: 1112.3028].

[17] G. F. Giudice and R. Rattazzi, Theories with Gauge-Mediated Supersymmetry Breaking, Phys. Rept. 322 (1999) 419-499, [hep-ph/9801271].

[18] P. Meade, N. Seiberg, and D. Shih, General Gauge Mediation, Prog. Theor. Phys. Suppl. 177 (2009) 143, [arXiv:0801.3278].

[19] M. Buican, P. Meade, N. Seiberg, and D. Shih, Exploring General Gauge Mediation, JHEP 0903 (2009) 016, [arXiv:0812.3668].

[20] J. L. Evans, M. Ibe, S. Shirai, and T. T. Yanagida, A $125 \mathrm{GeV}$ Higgs boson and muon $g$ 2 in more generic gauge mediation, Phys. Rev. D85 (2012) 095004, [arXiv:1201.2611].

[21] Z. Kang, T. Li, T. Liu, C. Tong, and J. Yang, A heavy SM-like Higgs and a light stop from Yukawa-deflected gauge mediation, Phys. Rev. D86 (2012) 095020, [arXiv: 1203.2336].

[22] P. Grajek, A. Mariotti, and D. Redigolo, Phenomenology of general gauge mediation in light of a $125 \mathrm{GeV}$ Higgs, JHEP 07 (2013) 109, [arXiv: 1303.0870].

[23] Particle Data Group Collaboration, J. Beringer et al., Review of particle physics, Phys. Rev. D86 (2012) 010001.

[24] H. Goldberg, Constraint on the photino mass from cosmology, Phys. Rev. Lett. 50 (1983) 1419.

[25] J. R. Ellis, J. S. Hagelin, D. V. Nanopoulos, K. A. Olive, and M. Srednicki Nucl. Phys. B 238 (1984) 453.

[26] J. R. Ellis, J. S. Hagelin, D. V. Nanopoulos, and M. Srednicki Phys. Lett. B127 (1983) 233.

[27] L. Ibáñez Phys. Lett. B 137 (1984) 160.

[28] J. Hagelin, G. Kane, and S. Raby Nucl. Phys. B 241 (1994) 638.

[29] T. Falk, K. A. Olive, and M. Srednicki, Heavy sneutrinos as dark matter, Phys. Lett. B339 (1994) 248, [hep-ph/9409270].

[30] K. Tamvakis and D. Wyler Phys. Lett. B112 (1982) 451. 
[31] H. P. Nilles and S. Raby Nucl. Phys. B198 (1982) 102.

[32] L. Covi, Gravitino dark matter confronts lhc, J. Phys. Conf. Ser. 485 (2014) 012002.

[33] SNO Collaboration Phys. Rev. Lett 92 (2004) 1022004.

[34] B. A. Campbell, S. Davidson, J. R. Ellis, and K. A. Olive, Cosmological baryon asymmetry constraints on extensions of the standard model, Phys. Lett. B256 (1991) 484-490.

[35] W. Buchmueller, L. Covi, K. Hamaguchi, A. Ibarra, and T. Yanagida, Gravitino dark matter in R-parity breaking vacua, JHEP 03 (2007) 037, [hep-ph/0702184].

[36] G. Giudice and A. Masiero, A natural solution to the mu problem in supergravity theories, Phys. Lett. B206 (1988) 480-484.

[37] M. Grefe, Neutrino Signals from Gravitino Dark Matter with Broken R-parity, arXiv:1111.6041.

[38] J. Pradler, Electroweak Contributions to Thermal Gravitino, arXiv:0708.2786.

[39] M. Grefe, Unstable Gravitino Dark Matter - Prospects for Indirect and Direct Detection, arXiv:1111.6779.

[40] M. Garny, A. Ibarra, D. Tran, and C. Weniger, Gamma-Ray Lines from Radiative Dark Matter Decay, JCAP 1101 (2011) 032, [arXiv:1011.3786].

[41] M. Garny, A. Ibarra, and S. Vogl, Dark matter annihilations into two light fermions and one gauge boson: General analysis and antiproton constraints, JCAP 1204 (2012) 033, [arXiv: 1112.5155].

[42] M. Garny, A. Ibarra, and D. Tran, Constraints on Hadronically Decaying Dark Matter, JCAP 1208 (2012) 025, [arXiv: 1205.6783].

[43] R. Barbier, C. Berat, M. Besancon, M. Chemtob, A. Deandrea, et al., R-parity violating supersymmetry, Phys.Rept. 420 (2005) 1-202, [hep-ph/0406039].

[44] A. Y. Smirnov and F. Vissani, Upper bound on all products of R-parity violating couplings lambda-prime and lambda-prime-prime from proton decay, Phys.Lett. B380 (1996) 317-323, [hep-ph/9601387].

[45] N. Fornengo, L. Maccione, and A. Vittino, Constraints on particle dark matter from cosmic-ray antiprotons, JCAP 1404 (2014) 003, [arXiv: 1312.3579].

[46] A. Ibarra, A. S. Lamperstorfer, and J. Silk, Dark matter annihilations and decays after the AMS-02 positron measurements, Phys.Rev. D89 (2014) 063539, [arXiv:1309.2570]. 
[47] LAT Collaboration, M. Ackermann et al., Fermi LAT Search for Dark Matter in Gamma-ray Lines and the Inclusive Photon Spectrum, Phys.Rev. D86 (2012) 022002, [arXiv: 1205.2739].

[48] Fermi-LAT Collaboration, M. Ackermann et al., Search for Gamma-ray Spectral Lines with the Fermi Large Area Telescope and Dark Matter Implications, Phys.Rev. D88 (2013) 082002, [arXiv: 1305.5597].

[49] CMS Collaboration, S. Chatrchyan et al., The CMS experiment at the CERN LHC, JINST 3 (2008) S08004.

[50] S. Bobrovskyi, W. Buchmuller, J. Hajer, and J. Schmidt, Quasi-stable neutralinos at the LHC, JHEP 1109 (2011) 119, [arXiv:1107.0926].

[51] CMS Collaboration, S. Chatrchyan et al., Searches for long-lived charged particles in $p p$ collisions at $\sqrt{s}=7$ and 8 TeV, JHEP 1307 (2013) 122, [arXiv: 1305.0491].

[52] CMS Collaboration, P. Lujan et al., Search for long-lived particles at CMS, arXiv:1411.5939.

[53] CMS Collaboration, Search for Pair-production of First Generation Scalar Leptoquarks in pp Collisions at sqrt $s=8 \mathrm{TeV}$, Tech. Rep. CMS-PAS-EXO-12-041, CERN, Geneva, 2014.

[54] ATLAS Collaboration, G. Aad et al., Search for direct third-generation squark pair production in final states with missing transverse momentum and two b-jets in $\sqrt{s}=8$ TeV pp collisions with the ATLAS detector, JHEP 1310 (2013) 189, [arXiv:1308.2631].

[55] ATLAS Collaboration, G. Aad et al., Search for direct pair production of the top squark in all-hadronic final states in proton-proton collisions at $\sqrt{s}=8 \mathrm{TeV}$ with the ATLAS detector, JHEP 1409 (2014) 015, [arXiv: 1406.1122].

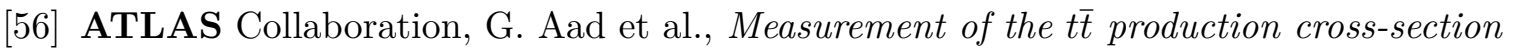
using e $\mu$ events with $b$-tagged jets in pp collisions at $\sqrt{s}=7$ and $8 \mathrm{TeV}$ with the ATLAS detector, Eur.Phys.J. C74 (2014), no. 10 3109, [arXiv:1406.5375].

[57] D. Hooper and S. Profumo, Dark matter and collider phenomenology of universal extra dimensions, Phys. Rept. 453 (2007) 29-115, [hep-ph/0701197].

[58] D. Feldman, Z. Liu, and P. Nath, The stueckelberg z' extension with kinetic mixing and milli-charged dark matter from the hidden sector, Phys. Rev. D75 (2007) 115001, [hep-ph/0702123].

[59] K. Cheung and T. C. Yuan, Hidden fermion as milli-charged dark matter in stueckelber g z' model, JHEP 03 (2007) 120, [hep-ph/0701107]. 
[60] J. W. Moffat, Modified gravity or dark matter?, arXiv:1101.1935.

[61] S. van den Bergh, A Short History of the Missing Mass and Dark Energy Paradigms, astro-ph/0005314.

[62] S. van den Bergh, The Early History of Dark Matter, astro-ph/9904251.

[63] K. Freese, Review of Observational Evidence for Dark Matter in the Universe and in upcoming searches for Dark Stars, astro-ph/0812.4005.

[64] A. H. G. Peter, Dark Matter: A Brief Review, astro-ph.Co/1201.3942.

[65] A. D. Dolgov, Introduction to Cosmology, hep-ph/0907.0668.

[66] S. Dodelson, Modern Cosmology. Academic Press (Elsevier), 2003.

[67] M. Trodden, Baryogenesis and leptogenesis, hep-ph/0411301.

[68] M. Taoso, G. Bertone, and A. Masiero, Dark Matter Candidates: A Ten-Point Test, arXiv:0711.4996.

[69] G. Bertone, D. Hooper, and J. Silk, Particle Dark Matter: Evidence, Candidates and Constraints, Phys.Rept. 405 (2005) 279-390, [0404175].

[70] G. Bertone, Particle Dark Matter - Observations, Models and Searches. Cambridge U. Press, 2010.

[71] F. Zwicky, Spectral displacement of extra galactic nebulae, Helv. Phys. Acta. 6 (1933) $110-127$.

[72] S. Smith, The mass of the virgo cluster, Astrophysical Journal 83 (1936) 23.

[73] A. S. Sharov and I. D. Novikov, Edwin Hubble, The Discoverer of the Big Bang Universe. Cambridge University Press, 2005.

[74] The sao/nasa astrophysics data system, . http://adswww.harvard.edu/.

[75] J. C. Adams, On the Perturbations of Uranus. Appendices to various nautical almanacs between the years 1834 and 1854 - W. Clowes \& Sons, 1846.

[76] R. A. Sampson, A description of Adams's manuscripts on the perturbations of Uranus, vol. 54. Memoirs of the Royal Astronomical Society, 1904.

[77] K. G. Begeman, B. A. H., and S. R. H., Extended rotation curves of spiral galaxies Dark haloes and modified dynamics, MNSRAS 249 (1991) 523.

[78] J. A. Tyson, G. P. Kochanski, and I. P. Dell'Antonio, Detailed Mass Map of CL0024+1654 from Strong Lensing, Astrophys. J. 498 (1998) L107, [9801193]. 
[79] M. Markevitch, Chandra observation of the most interesting cluster in the Universe, ESA Spec.Publ 604 (2006) 723, [astro-ph/0511345].

[80] Planck Collaboration, P. A. R. Ade et al., Planck Collab. 2015 XIII. Cosmological parameters, arXiv:1502.0158.

[81] J. S. Bullock, Notes on the missing satellites problem, arXiv:1009.4505.

[82] N. I. Libeskind et al., Cold versus Warm Dark Matter simulations of a galaxy group, Publ. Astron. Soc. Austral 30 (2013) 29, [arXiv:1305.5557].

[83] A. Zhitnitsky, Cold Dark Matter as Compact Composite Objects, Phys. Rev. D74 (2006) 043515, [astro-ph/0603064].

[84] B. D. Fields, P. Molaro, and S. Sarkar, Big Bang Nucleosynthesis, PDG 2013 (2013) [arXiv:1412.1408].

[85] B. Ratra and P. J. E. Peebles, Cosmological consequences of a rolling homogeneous scalar field, Phys. Rev. D37 (1988) 3406.

[86] B. Jain and J. Khoury, Cosmological tests of gravity, Annals of Physics 325 (2010) 1479-1516.

[87] E. Aver, K. A. Olive, and E. D. Skillman, An MCMC determination of the primordial helium abundance, JCAP 04 (2012) 004, [arXiv: 1112.3713].

[88] K. A. Olive, P. Petitjean, E. Vangioni, and J. Silk, Higher D or Li: Probes of Physics beyond the Standard Model, Mon. Not. Roy. Astron. Soc. 426 (2012) 1427, [arXiv: 1203.5701].

[89] Planck Collaboration, P. A. R. Ade et al., Planck Collab. 2013 XVI, JHEP 03 (2011) 024, [arXiv: 1303.5076].

[90] M. Kowalski et al., Improved Cosmological Constraints from New, Old and Combined Supernova Datasets, Astrophys. J. 686 (2008) 749-778, [arXiv:0804.4142].

[91] M. Fukugita and T. Yanagida, Baryogenesis without grand unification, Phys. Lett. B174 (1986) 45

[92] W. Buchmueller, P. Di Bari, and M. Plumacher, Leptogenesis for pedestrians, Ann. Phys. 315 (2005) 305-351, [hep-ph/0401240].

[93] S. Davidson and I. A, A lower bound on the right-handed neutrino mass from leptogenesis, Phys. Lett B535 (2002) 25-32, [hep-ph/0202239].

[94] J. K. Griest, M. Kamionkowski, and M. S. Turner Phys. Rev. Lett 64 (1990) 615. 
[95] G. Jungman, M. Kamionkowski, and K. Griest, Supersymmetric dark matter, Phys. Rept. 267 (1996) 195, [hep-ph/9506380].

[96] K. Griest and D. Seckel Phys. Rev. D43 (1991) 3191.

[97] G. Binetruy, P. Girardi and P. Salati Nucl. Phys. B237 (1984) 285.

[98] J. L. Feng, A. Rajaraman, and F. Takayama, Superweakly interacting massive particles, Phys.Rev.Lett. 91 (2003) 011302, [hep-ph/0302215].

[99] K. Jedamzik, Big bang nucleosynthesis constraints on hadronically and electromagnetically decaying relic neutral particles, Phys. Rev. D74 (2006) 103509, [hep-ph/0604251].

[100] R. H. Cyburt et al., Nucleosynthesis constraints on a massive gravitino in neutralino dark matter scenarios, JCAP 0910 (2009) 021, [arXiv:0907.5003].

[101] K. Jedamzik and M. Pospelov, Big bang nucleosynthesis and particle dark matter, New J. Phys. 11 (2009) 105028, [arXiv:0906.2087].

[102] L. J. Hall, K. Jedamzik, J. March-Russell, and S. M. West, Freeze-In Production of FIMP Dark Matter, JHEP 1003 (2010) 080, [arXiv:0911.1120].

[103] X. Chu, T. Hambye, and M. H. Tytgat, The Four Basic Ways of Creating Dark Matter Through a Portal, JCAP 1205 (2012) 034, [arXiv:1112.0493].

[104] C. Berger, L. Covi, S. Kraml, and F. Palorini, The number density of a charged relic, JCAP 10 (2008) 005, [arXiv: 0807.0211].

[105] K. Kohri and Y. Santoso, Cosmological scenario of stop nlsp with gravitino lsp and the cosmic lithium problem, Phys. Rev. D79 (2009) 043514, [arXiv:0811.1119].

[106] J. L. Diaz-Cruz, J. R. Ellis, K. A. Olive, and Y. Santoso, On the feasibility of a stop nlsp in gravitino dark matter scenarios, JHEP 05 (2007) 003, [hep-ph/0701229].

[107] J. Alwall, J. L. Feng, J. Kumar, and S. Su, Dark matter-motivated searches for exotic 4 th generation quarks in Tevatron and early LHC data, Phys. Rev. D81 (2010) 114027, [arXiv: 1002.3366].

[108] Y. Katz and D. Shih, Light stop NLSPs at the Tevatron and LHC, JHEP 08 (2011) 049, [arXiv: 1106.0030].

[109] C. Kilic and B. Tweedie, Cornering light stops with dileptonic $m_{T 2}$, JHEP 04 (2013) 110, [arXiv:1211.6106].

[110] Y. Bai, A. Katz, and B. Tweedie, Pulling out all the stops: searching for rpv susy with stop-jets, JHEP 01 (2014) 040, [arXiv:1309.6631]. 
[111] Z. Marshall, B. A. Ovrut, A. Purves, and S. Spinner, Lsp squark decays at the lhc and theneutrino mass hierarchy, Phys. Rev. D90 (2014) 015034, [arXiv:1402.5434].

[112] M. Asano, H. Kim, R. Kitano, and Y. Shimizu, Natural supersymmetry at the LHC, JHEP 12 (2010) 019, [arXiv: 1010.0692].

[113] C. Brust, A. Katz, S. Lawrence, and R. Sundrum, SUSY, the third generation and the LHC, JHEP 03 (2012) 103, [arXiv:1110.6670].

[114] M. Papucci, J. T. Ruderman, and A. Weiler, Natural SUSY endures, JHEP 09 (2012) 035, [arXiv: 1110.6926].

[115] K. Ishiwata, T. Ito, and T. Moroi, Long-lived unstable superparticles at the LHC, Phys. Lett. B669 (2008) 28, [arXiv:0807.0975].

[116] P. W. Graham, D. E. Kaplan, S. Rajendran, and P. Saraswat, Displaced supersymmetry, JHEP 07 (2012) 149, [arXiv: 1204.6038].

[117] CMS Collaboration, Search for heavy stable charged particles in pp collisions at $\sqrt{s}=7$ TeV, JHEP 03 (2011), no. CMS-EXO-10-011 024, [arXiv: 1101.1645].

[118] ATLAS Collaboration, Search for stable hadronising squarks and gluinos with the ATLAS experiment at the LHC, Phys. Lett. B 701 (2011) 1, [arXiv:1103.1984].

[119] CMS Collaboration, Search for heavy long-lived charged particles in pp collisions at $\sqrt{s}=7$ TeV, Phys. Lett B 713 (2012) 408, [arXiv:1205.0272].

[120] CMS Collaboration, S. Chatrchyan et al., Search for stopped long-lived particles produced in pp collisions at $\sqrt{s}=7 \mathrm{TeV}$, JHEP 1208 (2012) 026, [arXiv:1207.0106].

[121] ATLAS Collaboration, Search for long-lived, multi-charged particles in pp collisions at $\sqrt{s}=7 \mathrm{TeV}$ using the ATLAS detector, Phys. Lett. B 722 (2013) 305, [arXiv: 1301.5272].

[122] ATLAS Collaboration, G. Aad et al., Search for long-lived stopped R-hadrons decaying out-of-time with pp collisions using the ATLAS detector, Phys.Rev. D88 (2013) 112003, [arXiv: 1310.6584$]$.

[123] S. Bobrovskyi, W. Buchmueller, J. Hajer, and J. Schmidt, Broken R-Parity in the Sky and at the LHC, JHEP 10 (2010) 061, [arXiv:1007.5007].

[124] P. Meade, M. Reece, and D. Shih, Long-lived neutralino NLSPs, JHEP 10 (2010) 067, [arXiv: 1006.4575$]$.

[125] S. Bobrovskyi, W. Buchmueller, J. Hajer, and J. Schmidt, Quasi-stable neutralinos at the LHC, JHEP 09 (2011) 119, [arXiv:1107.0926]. 
[126] M. Hirsch, W. Porod, and D. Restrepo, Collider signals of gravitino dark matter in bilinearly broken R-parity, JHEP 03 (2005) 062, [hep-ph/0503059].

[127] P. Ghosh, D. E. Lopez-Fogliani, V. A. Mitsou, C. Muñoz, and R. Ruiz de Austri, Probing the $\mu$-from- $\nu$ supersymmetric standard model with displaced multileptons from the decay of a Higgs boson at the LHC, Phys. Rev. D 88 (2013) 015009, [arXiv:1211.3177].

[128] L. Covi, M. Olechowski, S. Pokorski, K. Turzynski, and J. D. Wells, Supersymmetric mass spectra for gravitino dark matter with a high reheating temperature, JHEP 01 (2011) 033, [arXiv: 1009.3801].

[129] F. D. Steffen, Probing the reheating temperature at colliders and with primordial nucleosynthesis, Phys. Lett. B 669 (2008) 74, [arXiv:0806.3266].

[130] P. J. and F. D. Steffen, Thermal relic abundances of long-lived staus, Nucl. Phys. B 809 (2009) 318, [arXiv:0808.2462].

[131] M. Endo, K. Hamaguchi, and K. Nakaji, Probing high reheating temperature scenarios at the LHC with long-lived staus, JHEP 11 (2010) 004, [arXiv:1008.2307].

[132] J. Heisig and J. Kersten, Production of long-lived staus in the Drell-Yan process, Phys. Rev. D84 (2011) 115009, [arXiv:1106.0764].

[133] J. M. Lindert, F. D. Steffen, and M. K. Trenkel, Direct stau production at hadron colliders in cosmologically motivated scenarios, JHEP 08 (2011) 151, [arXiv: 1106.4005].

[134] J. Heisig and J. Kersten, Long-lived staus from strong production in a simplified model approach, Phys. Rev. D86 (2012) 055020, [arXiv: 1203.1581].

[135] J. Heisig, J. Kersten, B. Panes, and T. Robens, A survey for low stau yields in the MSSM, JHEP 04 (2014) 053, [arXiv:1310.2825].

[136] J. Heisig, Gravitino LSP and leptogenesis after the first LHC results, JCAP 04 (2014) 023, [arXiv: 1310.6352].

[137] L. Covi and S. Kraml, Collider signatures of gravitino dark matter with a sneutrino NLSP, JHEP 08 (2007) 015, [hep-ph/0703130].

[138] J. R. Ellis, K. A. Olive, and Y. Santoso, Sneutrino NLSP scenarios in the NUHM with gravitino dark matter, JHEP 10 (2008) 005, [arXiv: 0807.3736].

[139] A. Katz and B. Tweedie, Signals of a sneutrino (N)LSP at the LHC, Phys. Rev. D81 (2010) 035012, [arXiv:0911.4132].

[140] T. Figy, K. Rolbiecki, and Y. Santoso, Tau-sneutrino NLSP and multilepton signatures at the LHC, Phys. Rev. D82 (2010) 075016, [arXiv:1005.5136]. 
[141] L. Roszkowski, S. Trojanowski, K. Turzynski, and K. Jedamzik, Gravitino dark matter with constraints from Higgs boson mass and sneutrino decays, JHEP 03 (2013) 013, [arXiv: 1212.5587].

[142] S. Bobrovskyi, J. Hajer, and S. Rydbeck, Long-lived higgsinos as probes of gravitino dark matter at the LHC, JHEP 02 (2013) 133, [arXiv: 1211. 5584].

[143] W. Beenakker, M. Kraemer, T. Plehn, M. Spira, and P. Zerwas, Stop production at hadron colliders, Nucl. Phys. B515 (1998) 3, [hep-ph/9710451].

[144] J. Alwall, M. Herquet, F. Maltoni, O. Mattelaer, and T. Stelzer, MadGraph 5 : Going Beyond, JHEP 1106 (2011) 128, [arXiv:1106.0522].

[145] E. Nikolidakis and C. Smith, Minimal flavor violation, seesaw and R-parity, Phys. Rev. D77 (2008) 015021, [arXiv:0710.3129].

[146] C. Csáki, Y. Grossman, and B. Heidenreich, MFV SUSY: a natural theory for R-parity violation, Phys. Rev. D85 (2012) 095009, [arXiv:1111.1239].

[147] S. Gates Jr. and O. Lebedev, Searching for supersymmetry in hadrons, Phys. Lett. D477 (2000) 216, [hep-ph/9912362].

[148] M. Fairbairn et al., Stable massive particles at colliders, Phys. Rept. 438 (2007) [hep-ph/0611040].

[149] M. Kawasaki, K. Kohri, and T. Moroi, Big-bang nucleosynthesis and hadronic decay of long-lived massive particles, Phys. Rev. D71 (2005) 083502, [astro-ph/0408426].

[150] M. Kusakabe, T. Kajino, T. Yoshida, and G. Mathews, Effect of long-lived strongly interacting relic particles on big bang nucleosynthesis, Phys. Rev. D80 (2009) 103501, [arXiv: 0906.3516].

[151] K. Kohri, Primordial nucleosynthesis and hadronic decay of a massive particle with a relatively short lifetime, Phys. Rev. D64 (2001) 043515, [astro-ph/0103411].

[152] A. Sommerfeld, Atombau und Spektrallinien, Band 2. Vieweg \& Sohn, 1939.

[153] A. D. Sakharov, Zh. Eksp. Teor. Fiz. 18, 631 (1948) [Sov. Phys. Usp. 34, 375 (1991)].

[154] J. S. Schwinger, Particles, sources, and fields. Vol. 2. Addison-Wesley (Advanced book classics series), 1989.

[155] L. Covi, J. Kim, and L. Roszkowski, Axinos as cold dark matter, Phys. Rev. Lett 82 (1999) 4180, [hep-ph/9905212].

[156] T. Moroi, H. Murayama, and M. Yamaguchi, Cosmological constraints on the light stable gravitino, Phys. Lett B 303 (1993) 289. 
[157] M. Bolz, A. Brandenburg, and W. Buchmueller, Thermal production of gravitinos, Nucl. Phys. B 606 (2001) 518, [hep-ph/0012052].

[158] J. Pradler and F. Steffen, Thermal gravitino production and collider tests of leptogenesis, Phys. Rev. D 75 (2007) 023509, [hep-ph/0608344].

[159] C. Cheung, G. Elor, and L. Hall, Gravitino freeze-in, Phys. Rev. D 84 (2011) 115021, [arXiv: 1103.4394].

[160] WMAP Collaboration, E. Komatsu et al., Seven-Year Wilkinson Microwave Anisotropy Probe (WMAP) Observations: Cosmological Interpretation, Astrophys. J. Suppl. 192 (2011) 18, [arXiv: 1001.4538].

[161] W. Beenakker, R. Hopker, and M. Spira, PROSPINO: A Program for the production of supersymmetric particles in next-to-leading order QCD, hep-ph/9611232.

[162] ATLAS Collaboration, A. Georges et al., Searches for heavy long-lived charged particles with the ATLAS detector in proton-proton collisions at $\sqrt{s}=8 \mathrm{TeV}$, JHEP 1501 (2015) 068, [arXiv:1411.6795].

[163] A. Alloul, N. Christensen, C. Degrande, C. Duhr, and B. Fuks, FeynRules 2.0 - a complete toolbox for tree-level phenomenology, Comput. Phys. Commun. 185 (2014) 2250, [arXiv: 1310.1921].

[164] F. WEB http://feynrules.irmp.ucl.ac.be/wiki/ModelDatabaseMainPage.

[165] Fermi LAT Collaboration, A. A. Abdo et al., Fermi LAT search for photon lines from 30 to $200 \mathrm{GeV}$ and dark matter implications, Phys. Rev. Lett. 104 (2010) 091302, [arXiv: 1001.4836$]$.

[166] Fermi LAT Collaboration, A. A. Abdo et al., The spectrum of the isotropic diffuse gamma-ray emission derived from first-year Fermi Large Area Telescope data, Phys. Rev. Lett. 104 (2010) 101101, [arXiv:1002.3603].

[167] G. Vertongen and C. Weniger, Hunting dark matter gamma-ray lines with the Fermi LAT, JCAP 05 (2011) 027, [arXiv:1101.2610].

[168] A. de Gouvêa, S. Gopalakrishna, and W. Porod, Stop decay into right-handed sneutrino LSP at hadron colliders, JHEP 11 (2006) 050, [hep-ph/0606296].

[169] K. Y. Choi, D. Lopez-Fogliani, C. Muñoz, and R. de Austri, Gamma-ray detection from gravitino dark matter decay in the $\mu \nu S S M$, JCAP 03 (2010) 028, [arXiv:0906.3681].

[170] CMS Collaboration, Search for long-lived neutral particles decaying to dijets, Tech. Rep. CMS-PAS-EXO-12-038, CERN, Geneva, 2013. 
[171] Search for long-lived, heavy particles in final states with a muon and a multi-track displaced vertex in proton-proton collisions at sqrt $(s)=8 \mathrm{TeV}$ with the ATLAS detector., Tech. Rep. ATLAS-CONF-2013-092, CERN, Geneva, Aug, 2013.

[172] J. Alwall, R. Frederix, S. Frixione, V. Hirschi, F. Maltoni, et al., The automated computation of tree-level and next-to-leading order differential cross sections, and their matching to parton shower simulations, JHEP 1407 (2014) 079, [arXiv: 1405.0301].

[173] ATLAS Collaboration, G. Aad et al., Search for direct production of charginos, neutralinos and sleptons in final states with two leptons and missing transverse momentum in pp collisions at $\sqrt{s}=8 \mathrm{TeV}$ with the ATLAS detector, JHEP 1405 (2014) 071, [arXiv: 1403.5294].

[174] CMS Collaboration, V. Khachatryan et al., Searches for electroweak production of charginos, neutralinos, and sleptons decaying to leptons and W, Z, and Higgs bosons in pp collisions at $8 \mathrm{TeV}$, arXiv:1405.7570.

[175] P. J. Fox, R. Harnik, J. Kopp, and Y. Tsai, Missing Energy Signatures of Dark Matter at the LHC, Phys.Rev. D85 (2012) 056011, [arXiv:1109.4398].

[176] CMS Collaboration, Search for new physics in monojet events in pp collisions at $\operatorname{sqrt}(s)=8 \mathrm{TeV}$, Tech. Rep. CMS-PAS-EXO-12-048, CERN, Geneva, 2013.

[177] T. Behnke et al., The International Linear Collider Technical Design Report - Volume2: Physics, .

[178] M. Aicheler et al., A Multi-TeV Linear Collider Based on CLIC Technology: CLIC Conceptual Design Report, .

[179] E. Bulbul, M. Markevitch, A. Foster, R. K. Smith, M. Loewenstein, et al., Detection of An Unidentified Emission Line in the Stacked X-ray spectrum of Galaxy Clusters, Astrophys.J. 789 (2014) 13, [arXiv:1402.2301].

[180] A. Boyarsky, O. Ruchayskiy, D. Iakubovskyi, and J. Franse, An unidentified line in $X$-ray spectra of the Andromeda galaxy and Perseus galaxy cluster, arXiv:1402.4119.

[181] T. E. Jeltema and S. Profumo, Dark matter searches going bananas: the contribution of Potassium (and Chlorine) to the $3.5 \mathrm{keV}$ line, arXiv:1408.1699.

[182] S. Baek, P. Ko, and W.-I. Park, The $3.5 \mathrm{keV}$ X-ray line signature from annihilating and decaying dark matter in Weinberg model, arXiv:1405.3730.

[183] E. Dudas, L. Heurtier, and Y. Mambrini, Generating X-ray lines from annihilating dark matter, Phys.Rev. D90 (2014) 035002, [arXiv:1404.1927]. 
[184] K. N. Abazajian, Resonantly-Produced 7 keV Sterile Neutrino Dark Matter Models and the Properties of Milky Way Satellites, Phys.Rev.Lett. 112 (2014) 161303, [arXiv:1403.0954].

[185] S. Baek and H. Okada, 7 keV Dark Matter as X-ray Line Signal in Radiative Neutrino Model, arXiv:1403.1710.

[186] S. Patra, N. Sahoo, and N. Sahu, Dipolar dark matter in light of $3.5 \mathrm{keV} \mathrm{X-ray} \mathrm{Line,}$ Neutrino mass and LUX data, arXiv:1412.4253.

[187] T. Tsuyuki, Neutrino masses, leptogenesis, and sterile neutrino dark matter, Phys.Rev. D90 (2014) 013007, [arXiv: 1403.5053].

[188] S. K. Kang and A. Patra, keV Sterile Neutrino Dark Matter and Low Scale Leptogenesis, arXiv: 1412.4899.

[189] H. Okada and T. Toma, $3.55 \mathrm{keV}$ X-ray Line Signal from Excited Dark Matter in Radiative Neutrino Model, Phys.Lett. B737 (2014) 162-166, [arXiv:1404.4795].

[190] J. M. Cline, Y. Farzan, Z. Liu, G. D. Moore, and W. Xue, $3.5 \mathrm{keV} X$-rays as the "21 cm line" of dark atoms, and a link to light sterile neutrinos, Phys.Rev. D89 (2014) 121302, [arXiv: 1404.3729].

[191] K. P. Modak, $3.5 \mathrm{keV} \mathrm{X-ray} \mathrm{Line} \mathrm{Signal} \mathrm{from} \mathrm{Decay} \mathrm{of} \mathrm{Right-Handed} \mathrm{Neutrino} \mathrm{due} \mathrm{to}$ Transition Magnetic Moment, arXiv:1404.3676.

[192] R. Allahverdi, B. Dutta, and Y. Gao, keV Photon Emission from Light Nonthermal Dark Matter, Phys.Rev. D89 (2014) 127305, [arXiv: 1403.5717].

[193] D. J. Robinson and Y. Tsai, Dynamical framework for KeV Dirac neutrino warm dark matter, Phys.Rev. D90 (2014), no. 4 045030, [arXiv:1404.7118].

[194] W. Rodejohann and H. Zhang, Signatures of Extra Dimensional Sterile Neutrinos, Phys.Lett. B737 (2014) 81-89, [arXiv: 1407.2739].

[195] N. Haba, H. Ishida, and R. Takahashi, $\nu_{R}$ dark matter-philic Higgs for $3.5 \mathrm{keV} \mathrm{X-ray}$ signal, arXiv:1407.6827.

[196] T. Higaki, K. Sik Jeong, and F. Takahashi, The 7 keV axion dark matter and the X-ray line signal, Phys.Rev.Lett. B733 (2014) 25-31, [arXiv:1402.6965].

[197] D. P. Finkbeiner and N. Weiner, An X-Ray Line from eXciting Dark Matter, arXiv:1402.6671.

[198] J. Jaeckel, J. Redondo, and A. Ringwald, $3.55 \mathrm{keV}$ hint for decaying axionlike particle dark matter, Phys.Rev. D89 (2014), no. 10 103511, [arXiv:1402.7335]. 
[199] H. M. Lee, Magnetic dark matter for the X-ray line at 3.55 keV, Phys.Lett. B738 (2014) 118-122, [arXiv: 1404.5446].

[200] M. Cicoli, J. P. Conlon, M. C. D. Marsh, and M. Rummel, $3.55 \mathrm{keV}$ photon line and its morphology from a $3.55 \mathrm{keV}$ axionlike particle line, Phys.Rev. D90 (2014), no. 2 023540, [arXiv: 1403.2370].

[201] S. P. Liew, Axino dark matter in light of an anomalous X-ray line, JCAP 05 (2014) 044, [arXiv: 1403.6621$]$.

[202] J.-C. Park, S. C. Park, and K. Kong, X-ray line signal from 7 keV axino dark matter decay, Phys.Lett. B733 (2014) 217-220, [arXiv:1403.1536].

[203] K.-Y. Choi and O. Seto, X-ray line signal from decaying axino warm dark matter, Phys.Lett. B735 (2014) 92, [arXiv:1403.1782].

[204] J. P. Conlon and A. J. Powell, A $3.55 \mathrm{keV}$ line from DM $\rightarrow a \rightarrow \gamma$ : predictions for cool-core and non-cool-core clusters, arXiv:1406.5518.

[205] N. E. Bomark and L. Roszkowski, $3.5 \mathrm{keV}$ x-ray line from decaying gravitino dark matter, Phys.Rev. D90 (2014), no. 1 011701, [arXiv:1403.6503].

[206] S. Demidov and D. Gorbunov, SUSY in the sky or a keV signature of sub-GeV gravitino dark matter, Phys.Rev. D90 (2014), no. 3 035014, [arXiv:1404.1339].

[207] K. Nakayama, F. Takahashi, and T. T. Yanagida, The $3.5 \mathrm{keV} \mathrm{X-ray} \mathrm{line} \mathrm{signal} \mathrm{from}$ decaying moduli with low cutoff scale, Phys.Lett. B735 (2014) 338-339, [arXiv: 1403.1733].

[208] C.-W. Chiang and T. Yamada, $3.5 \mathrm{keV} \mathrm{X-ray} \mathrm{line} \mathrm{from} \mathrm{nearly-degenerate} \mathrm{WIMP} \mathrm{dark}$ matter decays, JHEP 1409 (2014) 006, [arXiv: 1407.0460].

[209] B. Shuve and I. Yavin, Dark matter progenitor: Light vector boson decay into sterile neutrinos, Phys.Rev. D89 (2014), no. 11 113004, [arXiv:1403.2727].

[210] C. Kolda and J. Unwin, X-ray lines from R-parity violating decays of keV sparticles, Phys.Rev. D90 (2014) 023535, [arXiv:1403.5580].

[211] B. Dutta, I. Gogoladze, R. Khalid, and Q. Shafi, $3.5 \mathrm{keV} X$-ray line and R-Parity Conserving Supersymmetry, JHEP 1411 (2014) 018, [arXiv:1407.0863].

[212] F. S. Queiroz and K. Sinha, The Poker Face of the Majoron Dark Matter Model: LUX to keV Line, Phys.Lett. B735 (2014) 69-74, [arXiv:1404.1400].

[213] C.-Q. Geng, D. Huang, and L.-H. Tsai, X-ray Line from the Dark Transition Electric Dipole, JHEP 1408 (2014) 086, [arXiv:1406.6481]. 
[214] J. M. Cline and A. R. Frey, Nonabelian dark matter models for $3.5 \mathrm{keV} \mathrm{X-rays,} \mathrm{JCAP}$ 1410 (2014), no. 10 013, [arXiv: 1408.0233].

[215] R. Krall, M. Reece, and T. Roxlo, Effective field theory and keV lines from dark matter, JCAP 1409 (2014) 007, [arXiv: 1403.1240].

[216] A. Falkowski, Y. Hochberg, and J. T. Ruderman, Displaced Vertices from X-ray Lines, arXiv:1409.2872.

[217] D. Malyshev, A. Neronov, and D. Eckert, Constraints on $3.55 \mathrm{keV}$ line emission from stacked observations of dwarf spheroidal galaxies, Phys.Rev. D90 (2014), no. 10 103506, [arXiv: 1408.3531].

[218] M. E. Anderson, E. Churazov, and J. N. Bregman, Non-Detection of X-Ray Emission From Sterile Neutrinos in Stacked Galaxy Spectra, arXiv:1408.4115.

[219] E. Carlson, T. Jeltema, and S. Profumo, Where do the $3.5 \mathrm{keV}$ photons come from? A morphological study of the Galactic Center and of Perseus, arXiv:1411.1758.

[220] E. Bulbul et al., Comment on 'dark matter searches going bananas: the contribution of potassium (and chlorine) to the 3.5 kev line', arXiv:1409.4143.

[221] A. Boyarsky, J. Franse, D. Iakubovskyi, and O. Ruchayskiy, Comment on the paper 'dark matter searches going bananas: the contribution of potassium (and chlorine) to the 3.5 kev line' by t. jeltema and s. profumo, arXiv:1408.4388.

[222] J. L. Feng, A. Rajaraman, and F. Takayama, SuperWIMP dark matter signals from the early universe, Phys.Rev. D68 (2003) 063504, [hep-ph/0306024].

[223] T. Asaka, M. Shaposhnikov, and A. Kusenko, Opening a new window for warm dark matter, Phys.Lett. B638 (2006) 401-406, [hep-ph/0602150].

[224] M. Nemevsek, G. Senjanovic, and Y. Zhang, Warm Dark Matter in Low Scale Left-Right Theory, JCAP 1207 (2012) 006, [arXiv:1205.0844].

[225] CMS Collaboration, V. Khachatryan et al., Search for pair production of third-generation scalar leptoquarks and top squarks in proton-proton collisions at sqrt(s) $=8$ TeV, Phys.Lett. B739 (2014) 229, [arXiv:1408.0806].

[226] M. Frigerio and C. E. Yaguna, Sterile Neutrino Dark Matter and Low Scale Leptogenesis from a Charged Scalar, arXiv:1409.0659.

[227] M. Frigerio (private communication, 2015).

[228] CMS Collaboration, V. Khachatryan et al., Constraints on the Higgs boson width from off-shell production and decay to Z-boson pairs, Phys.Lett. B736 (2014) 64, [arXiv: 1405.3455]. 
[229] ATLAS Collaboration, G. Aad et al., Search for direct top squark pair production in events with a $Z$ boson, $b$-jets and missing transverse momentum in $\operatorname{sqrt}(s)=8 \mathrm{TeV} p p$ collisions with the ATLAS detector, Eur.Phys.J. C74 (2014), no. 6 2883, [arXiv: 1403.5222].

[230] M. Blennow, E. Fernandez-Martinez, O. Mena, J. Redondo, and P. Serra, Asymmetric Dark Matter and Dark Radiation, JCAP 1207 (2012) 022, [arXiv:1203.5803].

[231] E. Di Valentino, A. Melchiorri, and O. Mena, Dark radiation sterile neutrino candidates after Planck data, JCAP 1311 (2013) 018, [arXiv:1304.5981].

[232] Planck Collaboration, P. Ade et al., Planck 2013 results. XVI. Cosmological parameters, Astron.Astrophys. 571 (2014) A16, [arXiv:1303.5076].

[233] S. Galli, K. Benabed, F. Bouchet, J.-F. Cardoso, F. Elsner, et al., CMB Polarization can constrain cosmology better than CMB temperature, Phys.Rev. D90 (2014), no. 6 063504, [arXiv:1403.5271].

[234] A. Boyarsky, A. Neronov, O. Ruchayskiy, and M. Shaposhnikov, Constraints on sterile neutrino as a dark matter candidate from the diffuse x-ray background, Mon.Not.Roy.Astron.Soc. 370 (2006) 213-218, [astro-ph/0512509].

[235] K. Petraki and A. Kusenko, Dark-matter sterile neutrinos in models with a gauge singlet in the Higgs sector, Phys.Rev. D77 (2008) 065014, [arXiv:0711.4646].

[236] A. Merle, V. Niro, and D. Schmidt, New Production Mechanism for keV Sterile Neutrino Dark Matter by Decays of Frozen-In Scalars, JCAP 1403 (2014) 028, [arXiv: 1306. 3996].

[237] E. Molinaro, C. E. Yaguna, and O. Zapata, FIMP realization of the scotogenic model, JCAP 1407 (2014) 015, [arXiv:1405.1259].

[238] A. Abada, G. Arcadi, and M. Lucente, Dark Matter in the minimal Inverse Seesaw mechanism, arXiv:1406.6556.

[239] S. Dodelson and L. M. Widrow, Sterile-neutrinos as dark matter, Phys.Rev.Lett. 72 (1994) 17-20, [hep-ph/9303287].

[240] X.-D. Shi and G. M. Fuller, A New dark matter candidate: Nonthermal sterile neutrinos, Phys.Rev.Lett. 82 (1999) 2832-2835, [astro-ph/9810076].

[241] M. Shaposhnikov, The nuMSM, leptonic asymmetries, and properties of singlet fermions, JHEP 0808 (2008) 008, [arXiv:0804.4542].

[242] G. Arcadi, L. Covi, and F. Dradi, LHC prospects for minimal decaying Dark Matter, JCAP 1410 (2014), no. 10 063, [arXiv:1408.1005]. 
[243] D. Boyanovsky, Clustering properties of a sterile neutrino dark matter candidate, Phys.Rev. D78 (2008) 103505, [arXiv:0807.0646].

[244] A. Merle and A. Schneider, Production of Sterile Neutrino Dark Matter and the $3.5 \mathrm{keV}$ line, arXiv:1409.6311.

[245] A. Merle and M. Totzauer, keV Sterile Neutrino Dark Matter from Singlet Scalar Decays: Basic Concepts and Subtle Features, arXiv:1502.0101.

[246] F. Tanedo, Monte Carlo with Madgraph 5 - personal notes and how-to guide, http://www.physics.uci.edu/tanedo/docs.html. (2011).

[247] J. Schuster, J. Thaler, and N. Toro, Chameleon : Event analysis software, http://v1.jthaler.net/olympics/software.html. 